\title{
Preparation, Functionalization, Modification, and Applications of Nanostructured Gold: A Critical Review
}

\author{
Muhammad Yaseen ${ }^{1}$, Muhammad Humayun ${ }^{2}$, Abbas Khan ${ }^{1, * \mathbb{D}}$, Muhammad Usman ${ }^{3} \mathbb{D}$, Habib Ullah $^{4} \mathbb{D}$, \\ Asif Ali Tahir ${ }^{5}$ (D) and Habib Ullah $5, *$ D
}

1 Department of Chemistry, Abdul Wali Khan University, Mardan 23200, KP, Pakistan; Yaseen.chemist@gmail.com

2 Wuhan National Laboratory for Optoelectronics, Huazhong University of Science and Technology, Wuhan 430074, China; 2017511018@hust.edu.cn

3 Center of Research Excellence in Nanotechnology (CENT), KFUPM, Dhahran 31261, Saudi Arabia; muhammadu@kfupm.edu.sa

4 State Key Laboratory of Advanced Technology for Materials Synthesis and Processing, Wuhan University of Technology, Wuhan 430074, China; habib_aas86@outlook.com

5 Environment and Sustainability Institute, University of Exeter, Penryn, Cornwall TR10 9FE, UK; A.Tahir@exeter.ac.uk

* Correspondence: abbas053@gmail.com (A.K.); hu203@exeter.ac.uk (H.U.)

Citation: Yaseen, M.; Humayun, M.; Khan, A.; Usman, M.; Ullah, H.; Tahir, A.A.; Ullah, H. Preparation,

Functionalization, Modification, and Applications of Nanostructured Gold: A Critical Review. Energies 2021, 14, 1278. https://doi.org/10.3390/ en14051278

Academic Editor: Ellen Stechel

Received: 18 January 2021

Accepted: 19 February 2021

Published: 25 February 2021

Publisher's Note: MDPI stays neutral with regard to jurisdictional claims in published maps and institutional affiliations.

Copyright: (c) 2021 by the authors. Licensee MDPI, Basel, Switzerland. This article is an open access article distributed under the terms and conditions of the Creative Commons Attribution (CC BY) license (https:/ / creativecommons.org/licenses/by/ $4.0 /)$.
Abstract: Gold nanoparticles (Au NPs) play a significant role in science and technology because of their unique size, shape, properties and broad range of potential applications. This review focuses on the various approaches employed for the synthesis, modification and functionalization of nanostructured $\mathrm{Au}$. The potential catalytic applications and their enhancement upon modification of Au nanostructures have also been discussed in detail. The present analysis also offers brief summaries of the major Au nanomaterials synthetic procedures, such as hydrothermal, solvothermal, sol-gel, direct oxidation, chemical vapor deposition, sonochemical deposition, electrochemical deposition, microwave and laser pyrolysis. Among the various strategies used for improving the catalytic performance of nanostructured $\mathrm{Au}$, the modification and functionalization of nanostructured $\mathrm{Au}$ produced better results. Therefore, various synthesis, modification and functionalization methods employed for better catalytic outcomes of nanostructured Au have been summarized in this review.

Keywords: nanomaterials; photocatalysis; pollutants degradation; solar fuel

\section{Introduction}

Nano is a Greek word which means small; particles with at least one dimension of less than $100 \mathrm{~nm}$ are called nanoparticles. Because of the large volume surface area, increased chemical reactivity or stability, enhanced mechanical strength, etc., nanoparticles have gained great popularity in the field of nanotechnology [1] and wide spreads applications in the field of electrochemistry, photochemical and biomedicine [2]. In general, nanoparticles have been classified into organic-, inorganic- and carbon-based. Inorganic metal nanoparticles are widely used in the preparation of nanoparticles, such as aluminium (Al), cadmium $(\mathrm{Cd})$, cobalt $(\mathrm{Co})$, copper $(\mathrm{Cu})$, gold $(\mathrm{Au})$, iron $(\mathrm{Fe})$, plum $(\mathrm{Pb})$, silver $(\mathrm{Ag})$ and zinc $(\mathrm{Zn})$. The Au nanoparticles were first prepared by Michael Faraday in 1856 [3,4]. Nanoparticles range in size from 1 to $8 \mu \mathrm{m}$ and have various shapes, including spherical ones, sub octahedron ones, octahedron, decahedron ones, icosahedral few twin ones, multiple twin crystal ones, tetrahedron, nano-triangles, hexagonals and nano-rods. Nanoparticles are different in size. The Au nanoparticles have attracted significant interest because of their high-volume ratio surface, low toxicity, excellent biocompatibility, optical, electronic and chemical properties [5-7]. Au nanoparticles are widely used in catalysis, optical molecular sensing, cancer treatment and as building blocks in nanotechnology [8]. Au colloid is used 
for surface modification of ideal electrodes due to its excellent stability and unique characteristics (including high biocompatibility maintaining the normal structure of attached proteins or enzymes and their enzyme function). The individual physical, chemical and Au nanoparticles optical properties can be innovative ways to control the transport pharmaceutical compounds and control [9]. The Au nanoparticles possess essential properties by functionalizing the surface with a change of ligands to improve the properties or bring about modifications in them that make the functionalized Au nanoparticles proper for new applications. Since the surface plasmon resonance (SPR) effect is caused by the reasonable driving on the surface of the nanoparticles, due to the interaction with the electromagnetic radiation of the appropriate wavelength, the strong absorption band and high luminous characteristics of Au can be improved due to surface plasmon resonance (SPR) impact [10]. The ultimate size and shape of the nanoparticles leads to its SPR optical absorption and scattering characteristics responsive to surrounding media and nanoparticles' aggregation condition. Rapid nanoparticle heating will cause formaldehyde oxidation in the air at environmental temperature [11,12].

Both due to the high absorption of visible light, ultraviolet light by Au nanoparticles, the oxidant reaction of synthetic dyes and other molecules, and the degradation of phenol and the selective oxidation of benzyl alcohol, due to the absorption of ultraviolet, $\mathrm{Au}$ NPs cause the $5 \mathrm{~d}$ electron to transition to the $6 \mathrm{sp}$ band (inter-band transition). It can be expected that ultraviolet rays will cause chemical reactions on Au NPs due to their high photon energy. This indicates that the complete solar spectrum can be used to drive the reaction with the new Au NPs photocatalyst. Although Au NPs have different absorption properties of ultraviolet and visible light [13]. The capacity of Au NPs to capture electrons appears to be determined by the location of the positive load in the configuration of the loaded Au NP electron bands. When absorbing visible light, a positive charge is generated in the 6sp band of $\mathrm{Au}$, which can oxidize molecules like dyes, methanol and $\mathrm{HCHO}$ [12]. $\mathrm{UV}$ absorption in the lower $5 \mathrm{~d}$ band of Au would be loaded positively and will oxidize molecules including phenol [12]. As Au NPs pick up ultraviolet radiation, 5d electrons are excited into the $6 \mathrm{sp}$ band and many of the excited electrons are high energy (see the green line or higher in Figure 1), and the oxygen molecules and carrier in the Au NPs intersections and helps the high-energy electrons of the $6 \mathrm{sp}$ band forming $\mathrm{O}^{-2}$ species. Then $\mathrm{O}_{2}$ is involved in $\mathrm{H}^{+}$to create other active substances such as radicals of $\mathrm{HO}_{2}$ or $\mathrm{OH}$ [14]. Therefore, a large surface photo-voltage response under ultraviolet light can be observed. Compared with the $6 \mathrm{sp}$ band, the positive charge remaining in the $5 \mathrm{~d}$ band has lower energy, so the affinity for electrons from the captured organic molecules is greater. This property can be used in two reaction schemes. First, under ultraviolet irradiation, the photocatalyst can oxidize compounds that cannot be oxidized under visible light, such as phenol. Second, the greater ability to trap electrons under ultraviolet light can be used to oxidize compounds into useful chemical intermediates. At the same time, the experimental conditions can be manipulated to prevent further oxidation and achieve selective oxidation with high selectivity. Both statistical process control (SPC) and transient photovoltage (TPV) measurements will detect when enormous electron transfer occurs. The visible light absorption by Au NPs the 6sp gain energy and transfer to the higher intraband energy levels. Through the collision of electrons and electrons, the plasma heats the electron gas to a high temperature (about $400-2000 \mathrm{~K}$ ) in $100 \mathrm{fs}$ or less [15]. On a given time scale, the $\mathrm{Au}$ electron gas obeys Fermi-Dirac diffusion at high temperatures. Then, the electron-photon interaction that shares the electron energy with the nanoparticle lattice occurs in the time range of $500 \mathrm{fs}$ to $10 \mathrm{ps}$. Therefore, it is believed that the electron gas maintains the excited "hot" state for up to $0.5-1$ ps. Very recently, Furube et al. [16] found that the transfer of electrons from $\mathrm{Au}$ NPs to $\mathrm{TiO}_{2}$ is very fast, taking less than 240 fs [17]. A small amount of excited electrons in Au may gain enough energy (above the green line in Figure 1) to be captured by the adsorbed $\mathrm{O}_{2}$ molecules on Au NPs under moderate visible light irradiation. The weak SPC signal demonstrate that majority of the excited electrons cannot be trapped by $\mathrm{O}_{2}$ molecules. However, the loaded Au NPs can attract the electrons of 
organic molecules on the NPs [18]. Due to light irradiation, the Sulforhodamine B (SRB) dye molecules get excited and then these excited molecules introduce their electrons to the substrate. The formation of $\mathrm{O}^{-2}$ species is affected by the additional dye sensitization of Au NPs by excited SRB molecules. In combination with SPR, the SRB effect on the Au NPs leads to high rate of dye degradation. By increasing the visible light intensity, positive charge increases and by gaining energy; a lot of electrons are seized by the $\mathrm{O}_{2}$ molecules. Molecular $\mathrm{O}_{2}$ is considered to be an oxidant that catalyzes the reaction. The selective oxidation of benzyl alcohol to benzaldehyde is an example of this scheme, which can only be observed under ultraviolet light. The main experimental observations include the band structure of Au NPs. Figure 1 shows the preliminary mechanism of photocatalysis using supported Au NPs. Considering that the $6 s p$ band overlaps the $5 \mathrm{~d}$ band in terms of energy scale, the proposed mechanism also provides the potential to turn on or turn off specific reactions by adjusting the wavelength of the irradiated light. This discovery reveals a new type of photocatalyst and a possible way through which sunlight can be used to drive various chemical reactions on the photocatalyst at ambient temperature for environmental purification and solar fuels production.

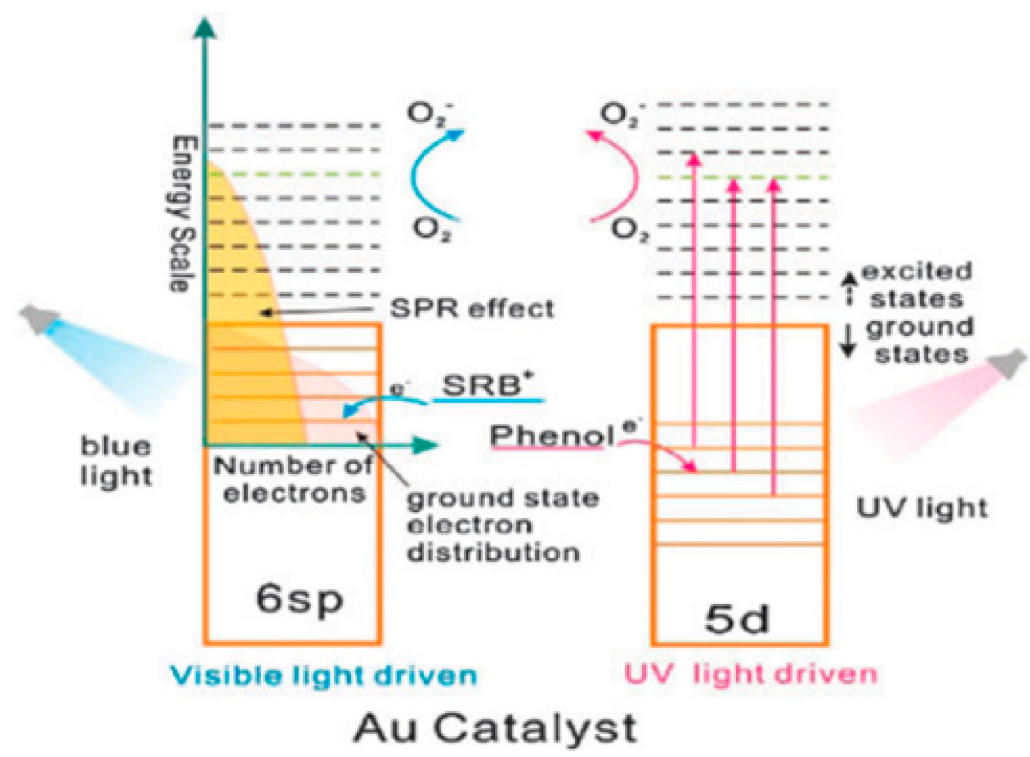

Figure 1. The energy band structure diagram of the supported Au NPs and the suggested mechanism of using the supported Au NPs for photocatalysis. Reproduce with permission from reference [14], RSC, 2010.

In this review, we attempted to summarize various methods for the preparation of nanostructured $\mathrm{Au}$ and its possible modification procedures for obtaining Au-based nanomaterials with different surface morphologies under different environment and examined their applications in several typical reactions in catalysis. This review also attempted to further highlight the basic understanding on the preparation, modification, functionalization and applications of nanostructured Au in catalysis. Furthermore, the basic mechanism of light absorption and simultaneous production of electron-hole, its trapping techniques and utilization have been described briefly.

\section{Synthetic Methods of Au NPs}

Au nanocrystals can be synthesized by a number of methods depending on its applications in various fields. Each method has its own advantages and disadvantages. Therefore, selection of an appropriate method is crucial because the growth of nanostructures, as well as their properties significantly depends on the methods of preparation. Such well-known techniques are hydrothermal, solvothermal, sol gel, direct oxidation, chemical vapor deposition, electrochemical deposition, sonochemistry, laser pyrolysis and 
microwave. Khumaeni Ali et al. [19] synthesized the Au NPs via pulse laser ablation technique with a simple wavelength laser, using the Pulse Laser (Nd: YAG Laser, 1064 nm, 7 ns, $30 \mathrm{~mJ}$ ), the low-power Neodymium yttrium aluminum grill is guided on a highly pure Au sheet (99.95 percent). They obtained dark-red color spherical shaped colloid Au NPs that were placed in deionized water. These results confirmed that using a low-power Nd:YAG laser, Au nanoparticles with high purity and identical size can b obtained. In another study, Eskandari-Nojedehi Maryam et al. [20] prepared Au nanoparticles via hydrothermal method in which Edible mushroom (Agaricus bisporus) extract functioned both as a reducing and stabilizing agent and $\mathrm{HAuCL}_{4} \cdot 3 \mathrm{H}_{2} \mathrm{O}$ solution was mixed. The results showed polyols and carbonyl groups in mushroom extract had effects on the formation of stable $\mathrm{Au}$ NPs. Further, they proposed an environmentally friendly and low-cost method relative to others chemical and/or physical NPs synthesis methods. The fabricated Au NPs showed great antifungal activity against Aspergillus flavus in comparison to the Aspergillus terreus. Errez-wing, Guti et al. [21] synthesized Au NPs via the microwave assisted method using 1-dodecanethiol. The results show that n-alkanethiol molecules not only act as passivation compounds, but also prevent crystal growth, and also interact to form a cubic ordered array of nanoparticles. The spontaneous formation of the superstructure of homogeneous $\mathrm{Au}$ NPs was also confirmed, and the new nano-engineering technology field of synthesizing nano-structured materials and high-productivity in a short time was expanded by this method. Sakai Toshio et al. [22] synthesized Au NPs through the sonochemical reduction method, with the help of hydrogen $\left(\mathrm{H}_{2}\right)$, the tetrachloride $\mathrm{Au}(\mathrm{III})$ ion is reduced in an aqueous solution of $\mathrm{Au}(\mathrm{III})$ tetrachloride tetrahydrate $\left(\mathrm{HAuCl}_{4} \cdot 4 \mathrm{H}_{2} \mathrm{O}\right)\left(\left[\mathrm{AuCl}_{4}\right]^{-}\right)$. There was no additional capping agent in the gas. They obtained the spherical Au NPs. Jameel Abdulghani and Rasha K. Hussain et al. [23] have synthesized the Au NPs through chemical reduction method by reducing (III) $\left(\mathrm{AuCl}^{4-}\right)$ isatine anions (1H-indole-2,3-dione) in the absence of all aqueous-solution reduction and dispersant agents. It was found that the synthesis of spherical Au NPs at room temperature increased by increasing concentration ratio of Is / Au (III) in the range 3.4-9.52. Babak Sadeghi et al. [24] synthesized the Au NPs via the green synthesis method by mixing the leaves extract of stevia rebadiauna (SR), which reduced the Au ions to Au NPs. The result confirmed the spherical and uniform distribution of the stable Au NPs with size ranges from 5 to $20 \mathrm{~nm}$. Lili Zhu et al. [25] prepared the Au NPs via the Brust-Schiffrin process in which tetraalkylammonium complexes of $\mathrm{Au}$ $\left([\mathrm{TOA}]\left[\mathrm{AuX}_{2}\right]\right)$ and $\mathrm{Au}$ thiolate ([TOA][AuSRX] and [TOA][Au(SR) $\left.)_{2}\right]$ ) soluble complexes were taken. The results confirmed that the complex [TOA][AuX $\left.\mathrm{T}_{2}\right]$ in the precursor and surplus thiol is reduced in to small Au NPs. If the concentration of the thiolate species in solution is greater then small and uniform nanoparticles will not form in this method. Hoo Xiao-Fen et al. [26] prepared the Au NPs by the seeding growth process. The Au particles with numerous sizes were synthesized by changing the synthetic parameters. The synthesized $\mathrm{Au}$ NPs are used to manufacture glucose sensors by using cyclic Voltammetry to test the electrocatalytic activity of Au NP/ITO electrodes. The results showed the highest electrocatalytic activity for glucose sensor with $30 \mathrm{~nm}$ Au NPs size compared to others. A. Ruivo et al. [27] reported the use of a sol-gel method to synthesize Au NP where the precursor of silica Sol-gel includes, under standard atmospheric conditions, $\mathrm{HAuCl}_{4} \cdot 3 \mathrm{H}_{2} \mathrm{O}$ and $[\mathrm{bmim}]\left[\mathrm{BF}_{4}\right]$. The results confirmed that, due to ionic liquid degradation, the Au NPs were produced in the sol-gel matrix at temperature in the range of $350{ }^{\circ} \mathrm{C}$ and $425^{\circ} \mathrm{C}$.

Masanori et al. [28] have mentioned in their review the synthesis of gold nanoparticles by the photochemical synthesis methods, i.e., direct photoreduction and photosensitization. They have shown and described there that such methods are more efficient relative to others for the nanoparticles and especially for gold nanoparticles. The direct photoreduction has taken advantage as it is without reducing agent and got applications in numerous mediums comprising polymer films, glasses, cells, etc. In addition the photosensitization has benefits over the photoreduction due to the fast and proficient production of metal NPs and flexibility of the excitation wave length depends on the sensitizer and not on the metal source. The various mechanisms regarding the synthesis of Au-nanoparticles via 
direct photoreduction and photosensitization have also been discussed and cited there. Lee et al. [29] synthesized the Au NP microstructures using two photons lithography from Au precursor containing poly(vinylpyrrolidone) (PVP) and ethylene glycol (EG), where EG indorses greater reduction rate of $\mathrm{Au}^{3+}$ via polyol reduction through two photon laser direct metal writing with characteristics for example NP size, particle density, surface roughness and consequently, plasmonic characteristics via modulating the PVP concentration in the precursor solution. They have also studied the gold nanoparticles within the microfluidic channel for SERS sensing of gaseous analytes. Izquierdo-Lorenzo et al. [30] prepared the gold nanoparticles by the radical mediated photoreduction and the fabrication in three dimensional microstructures comprising gold was done by two photon lithography. The synthesized structure showed plasmonic activity and outstanding properties as substrate for surface enhanced Raman scattering. These substrates can be used again for multiple measurements and capable it for practical uses such as integration into a microfluidics system for online analysis. Synthesis of Au nanoparticles while using multiphoton photoreduction approach was also carried by Ritacco and coworkers et al. [31]. They have also studied the physical phenomenon involved in the multi-photon direct laser writing (MP-DLW) of the gold nanoclusters through multi photon absorption in aqueous solution of metallic precursor, emphasizing the role in the main switch factors and the boundaries of this method simultaneously. They have also studied the effects of the ions and water diffusion on the structures of the gold nanoparticles, i.e., size, dispersion and density and their basic use in plasmonic phenomenon. It was found that the control on the Au NPs growth and clustering can be improved when the energy dose is delivered in multiple exposures.

The chemical method can generate Au NPs at low cost and provide repeatable results using the various chemical and biological methods described above (in terms of size and shape). However, the major disadvantage of the chemical synthesis method is that, toxic byproducts are produced which have environmental effects during large scale manufacturing. These toxic solvents and the hazardous chemical derivatives production in the above method are proven to be problematic for downstream biological uses of Au NPs [32-34]. In order to solve the problem of chemical method, biological based preparation method (using carbohydrates, lipids, nucleic acids or proteins, plants extracts, microorganism, etc.) was put forwarded which has developed a significant direction of present nano technology based research. The essence of colloidal Au NPs, including herbal components and derivatives, bacterias, fungi, algae, yeasts and viruses are effectively improved in the manufacture of colloidal Au NPs [35].

\section{Functionalization of Au NPs}

\subsection{Functionalization via Inorganic Moieties from $p$-Block}

Because of the covalent nature of the p-block component bonds in the periodic table, organic molecules, but also with elements from columns V to VII are prepared almost entirely from such components. Inorganic p-block moieties are seldom used to work $\mathrm{Au}$ NP. Three common molecular clusters, namely fullerenes (C60), carboranes and polyedral oligomeric silsesquioxanes have been widely studied in the literature (POSS). Their association with au NPs, mainly based on fullerene clusters, is defined in the article.

\subsubsection{Clusters of Fullerene (C60)}

Fullerene was found in 1985 and is widely regarded as a new allotropic type of carbon element (C60) [36]. C60 is one of the prevalent fullerenes commonly utilized for the design of composite materials because of its mechanical, spectral, structural and manageable functional properties [37]. Mathias Brust had the first comment on the $\mathrm{C}^{\prime} 0^{\prime}$ s connection to Au NPNPs, to the best of our knowledge, in 1998 [38]. In order to promote, C60 was used to help accrue free Au NPs in toluene. In the past, the functionality of C60 has been improved and only one covalent feature for C60 with fullerene for Au NP has been presented. In 2001, the initial thiolated fullerene functional Au NPs were described by Fujihara et al. [39,40]. In this, fullerene thiol and octanethiol resulted in stabilization 
of Au NPs (see Figure 2). K.G Thomas et al. [41] developed an analogous approach by using an alkyl chain between C60 and Au NPs in 2002 in comparison with Shon et al., who used an aromatic amino mercaptophenol (Figure 2) [42]. However, for Fujiwara, fullerene thiols surrounded particles with other alkane thiols are used as co-stabilizing means $\left(\mathrm{C}_{8} \mathrm{H}_{17} \mathrm{SH}\right.$ or $\left.\mathrm{Cl}_{2} \mathrm{H}_{25} \mathrm{SH}\right)$. The addition of the fullerene-thiol moiety was carried out by the ligand exchange method except for to Shon, who tried a direct process using a mixture of $\mathrm{C} 60-\mathrm{Ph}-\mathrm{SH}$ and $\mathrm{C}_{8} \mathrm{H}_{17} \mathrm{SH}$. For electrochemical or photoelectrical purposes, all these nanocomposites were prepared (Au-S-R-C60).

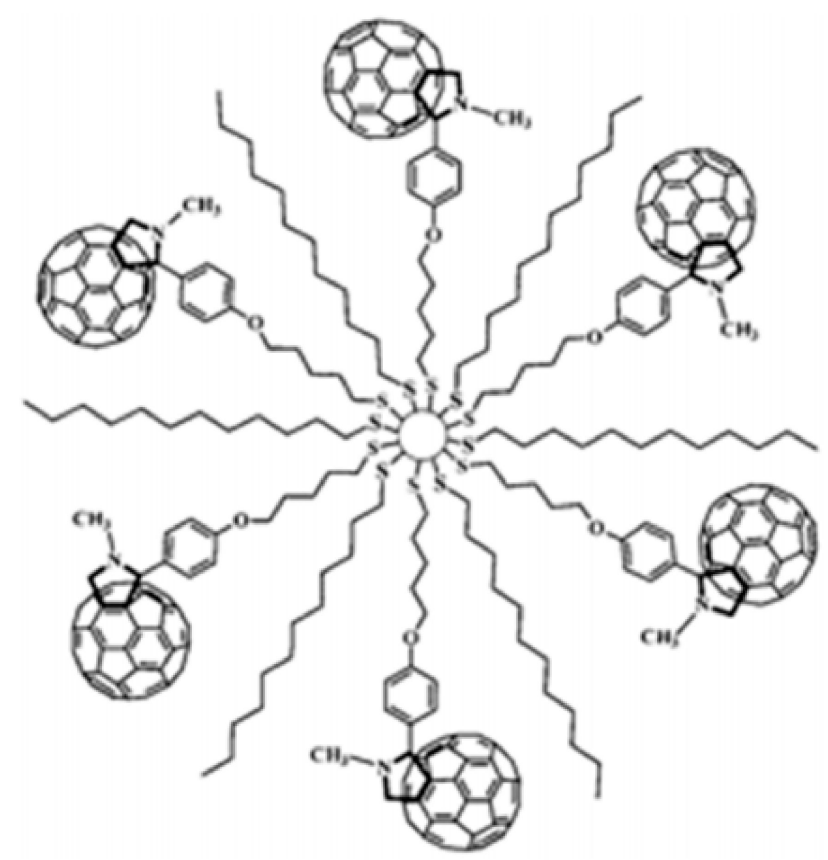

Figure 2. Scheme of a fullerene thiol-functional Au NP example. Reproduced with permission from ACS, 2002 [41].

Other advances concerning C60 and Au NPs related their blends with $\gamma$-cyclodextrines [43] to practice network collections or with porphyrins to scheme photovoltaic solar cells are depicted in Figure 3 [44]. Newly stabilized Au NPs with fullerene present numerous required styles [45] or with fulleropyrrolidine functionalized [46] were described.

\subsubsection{Carborane Clusters}

Because of their use in medicine, catalysis and materials science, polyhedral carborane clusters have been broadly considered $[47,48]$. Huge struggles have also been dedicated to reach an organised functionalization $[49,50]$. The cluster may in particular be directly controlled with one or two functional sulfhydryl groups through the -B-SH links [51,52]. Baše et al. $[53,54]$ demonstrated that only two courses define the straight Au NPs functionalization through carborane-thiol (see Figure 4). The collaboration between carborane-thiol collections and Au NPs was also studied. Electrochemical characteristics of these nanocomposites were also examined.

The potential uses of Au NPs based on carboran are very broad, and ion transport inspections via biological membranes [55] or uses for cancer [56] were remarkably advanced. 

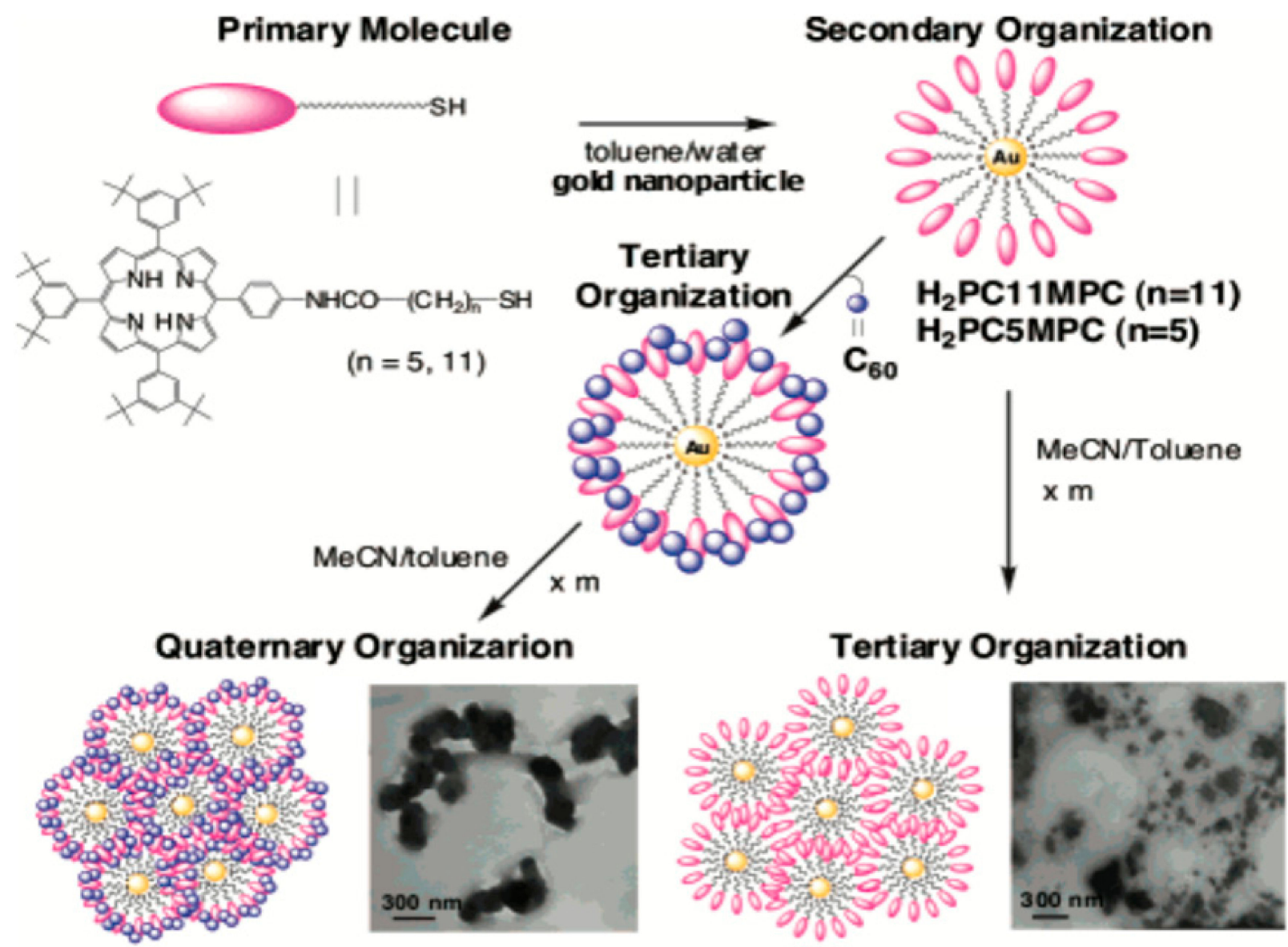

Figure 3. Nanocomposites based on porphyrin interaction and C60 on Au NP surface. ACS, 2003, reproduced with reference permission [44].
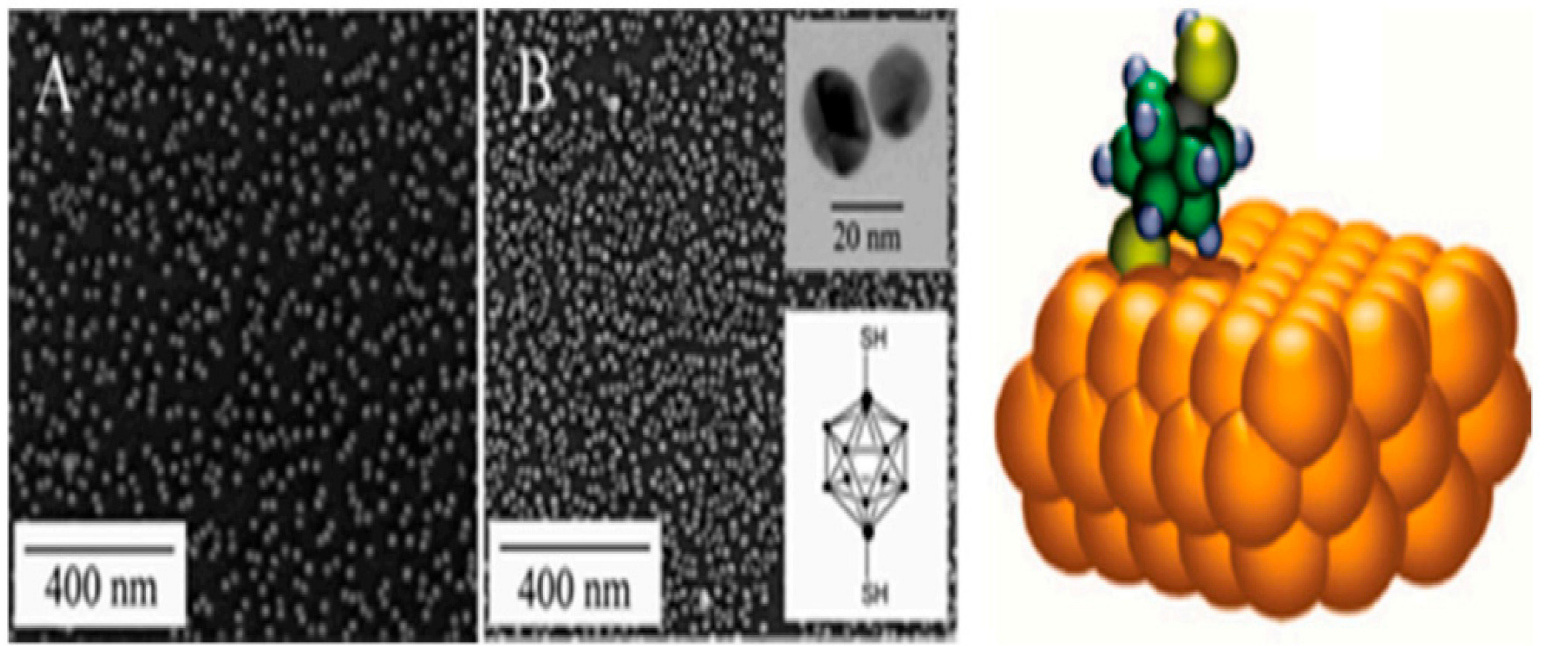

Figure 4. TEM images (A,B) of the Au NPs functioned on the capture of the same carboranethiol design on the Au(111) region, functional with the carboanethiol 1,12-(HS)2-1,12-C2 B10 H10. ACS, 2008, reproduced with reference permission [54].

\subsubsection{Clusters of POSS}

POSS clusters are commonly used in materials science particularly as mineral raw matter. Polyhedral oligomeric silsesquioxanes are clusters. They are called nanostructured by diameter $(1.5 \mathrm{~nm})$ and are easily controlled by one or many organic groups [57]. Actually, POSS was used for the first time by G. Schmid et al. [58] as a functional agent and alkylthiol groups were integrated successfully into the groups. The properties of the Au55Cl6 cluster 
NPs are another characteristic of this analysis. Rotello et al. [59] have made electrostatic or $\mathrm{H}_{2}$ connections necessary to bind POSS to Au NPs so that self-associations can be achieved. The method included Au NPs functioning with thymine functions (Thy-Au) for the $\mathrm{H}_{2}$ bonding communication and POSS functioning by di-amino-pyridine groups (POSS-DAP) (see Figure 5). The glory method for POSS relating to Thy-Au resulted in the selection of nanocomposites with a circular mixture produced by the non-polar POSS-crystalline DAP's filling. Another analysis included the inventory of POSS with 8 ammo-clusters and development of autonoassemblies by carboxylate coated au-NPs (octa-ammonium-POSS, OA-POSS) [60]. Over recent years, the use of POSS-based Au NPs has extended from colorimetric to reduction processes [61,62].

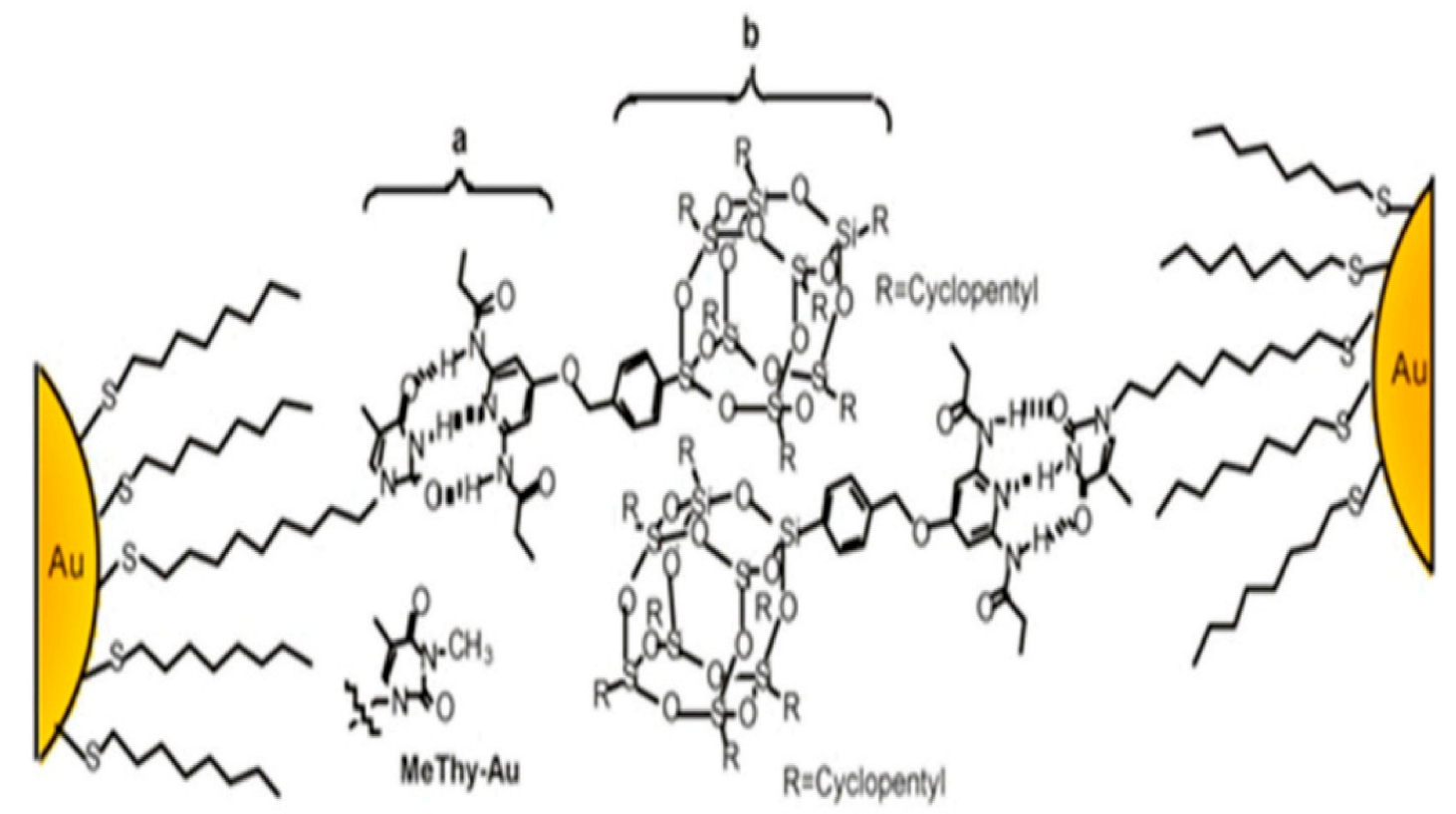

Figure 5. The interaction of Au NPs functionalized via thymine via three-point $\mathrm{H}_{2}$ bonding (a) and the POSS clusters (b). Reproduced with reference authorization [59], RSC, 2002.

\subsubsection{Silica}

Two aspects are simple to work and the fundamental colloidal stability of the resultant solution by its design in the modern nano composite framework. The probability of ligands desorbing from Au NPs is another interesting concept. Silica tends to be an excellent candidate in this pitch to prevent this difficult melting of particles. Due to chemically inertness, optically obvious and easily functionalize characteristics, the coating of $\mathrm{Au}$ NPs with $\mathrm{SiO}_{2}$ and also to limit the depth of coating [63,64]. Mulvaney and Liz-Marzán reported the synthesis of $\mathrm{Au}$ core $/ \mathrm{SiO}_{2}$ shell nanocomposites of manageable breadth. First, aminopropyl trimethoxysilane was used as the citrate-capped Au NPs. The Au surface has had strong amine contact, with alkoxysilane groups covering the surface entirely and siloxane groups reducing. The width of the silica coating was determined by the sodium silicate addition [65]. In many studies since this initial study, alternative methods for this synthetic method have been found $[66,67]$ to monitor the nanocomposite's optic properties $[68,69]$ or to work silica shell by polymers [70] or by chromophores to increase fluorescence (see Figure 6) [71]. 

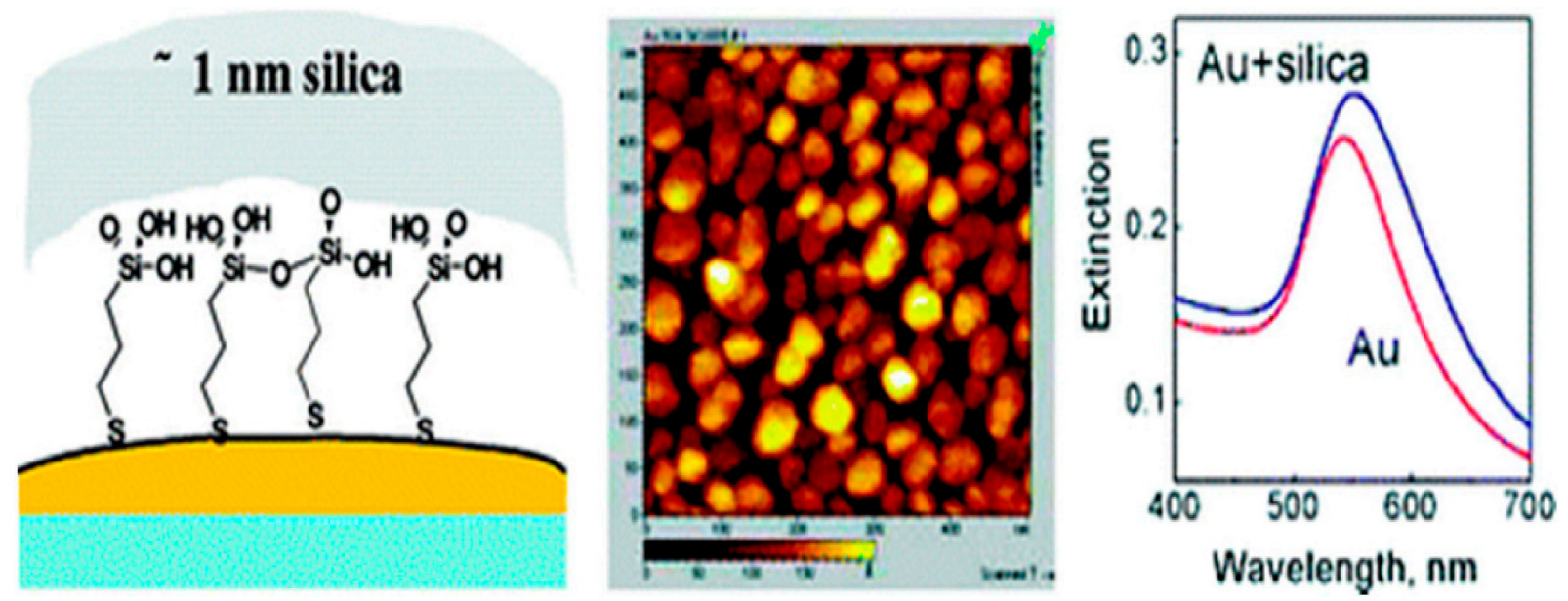

Figure 6. Schematic of coating $\mathrm{Au}$ NPs by means of $\mathrm{SiO}_{2}$ by a mercaptosilane group, using the $\mathrm{Au} / \mathrm{SiO} 2$ core/shell picture and UV-vis spectra. Reproduced by reference authorisation, ACS, 2007 [69].

Xia et al. [72] made a device through which first the Au core $/ \mathrm{SiO}_{2}$ shell was formed and then the functionalization of its surface was done by a second shell that is a polymer such as (poly(benzyl methacrylate). The $\mathrm{SiO}_{2}$ shell was dissolved by dispersing in aqueous solution of HF and hollow beads with movable Au cores were formed (see Figure 7).
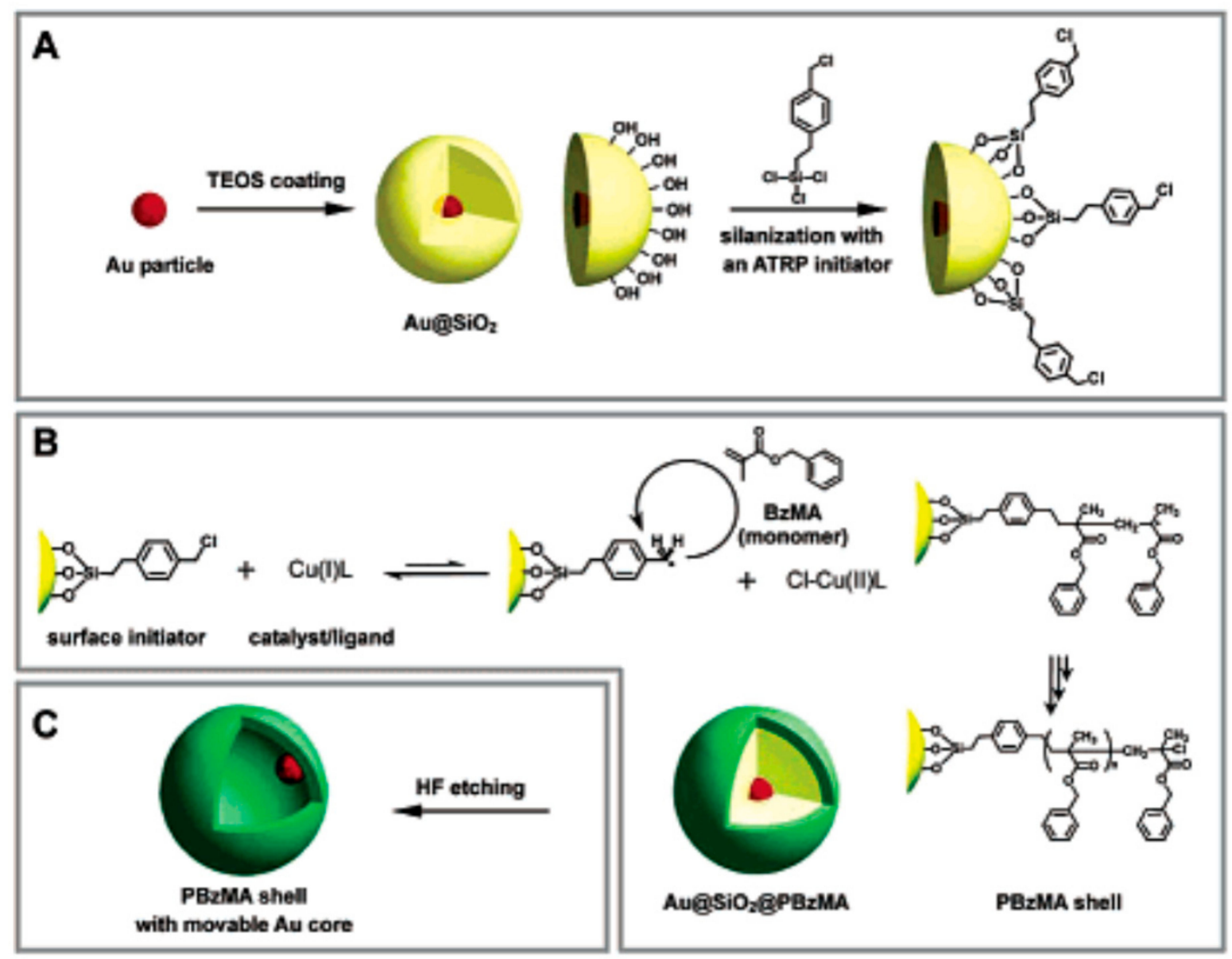

Figure 7. Cont. 

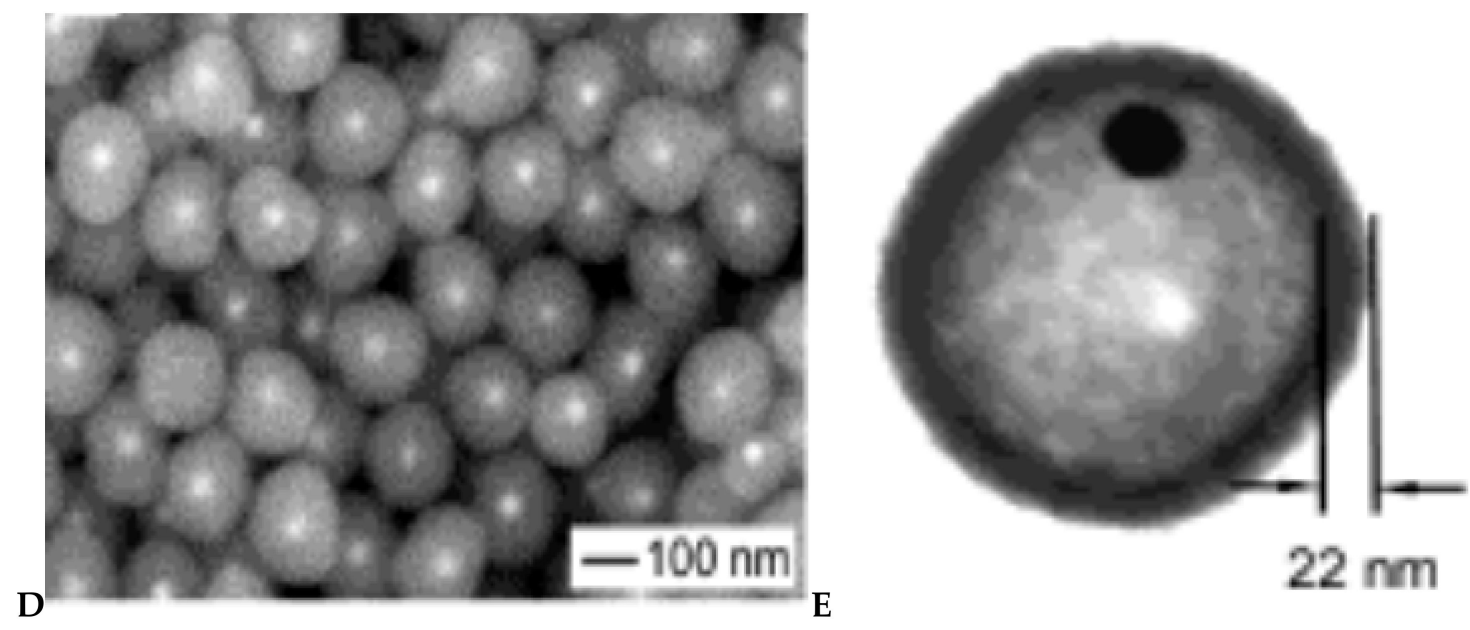

Figure 7. The hollow beads of PBzMA containing moving Au cores (C), the SEM image before HF andching (D) et the HF etching (E) image after HF and PBzMA particles, synthesized routes for Au@SiO $\mathrm{APBzMA} \mathrm{NP}_{2}$ (A,B). ACS, 2003, replicated by reference permission [72].

\subsubsection{Carbon Nanotubes}

The fullerenes and carbon nanotubes were discovered in 1991 [73]. These materials have then been thoroughly researched because of its peculiar structure, electrical and mechanical properties [74]. In the exact case of their nanocomposites, it is obvious that the nanotubes (NTs) will work through Au NPs because of their difference in size. Their composites are extremely favourable for numerous uses, such as optics, electronics, biosensors and catalysis [74,75]. For the functionalization of NTs by Au NPs, various methods have been examined. Thermal decomposition is the procedure used to reduce Au-III salt onto the carbon nanotubes surface [76]. Still, the key advances related the electrostatic and covalent port of Au NPs on NTs. Prefunctions of the NT surfaces were also important in the production of carboxylic acid groups, by oxidative action with $\mathrm{HNO}_{3}$ or $\mathrm{H}_{2} \mathrm{SO}_{4}-\mathrm{HNO}_{3}$ [77] Predictable organic reactions to NTs surfaces could previously occur because these carboxylic or other functional groups were present. An alternative method is to establish a boron nitride NTs surface amino groups (see Figure 8) [78]. The variance of the electrostatic charges of the NTs is additional to the occurrence of carboxylic acid groups. The anionic character of the NTs therefore allowed for adsorption of cationic polyelectrolyte cables and therefore contact with the Au NPs with negative charges [79,80].

Additional method by which Silica is coated with thiol or amino functions on the surface of NTs [81]. Silica coating functionality was described in a further step by Bottini et al. (see Figure 9) [82,83].

A substitute and sophisticated method was used to $\pi-\pi$ stack aromatic molecules such as pyrene to work NTs. This approach was used by Huang et al. with a 1-pyrenemethylamine as linker between NTs and Au NPs. Huang et al. [84] used this method as a liaison between NTs and Au NPs using 1-pyrene-mehylamine. 


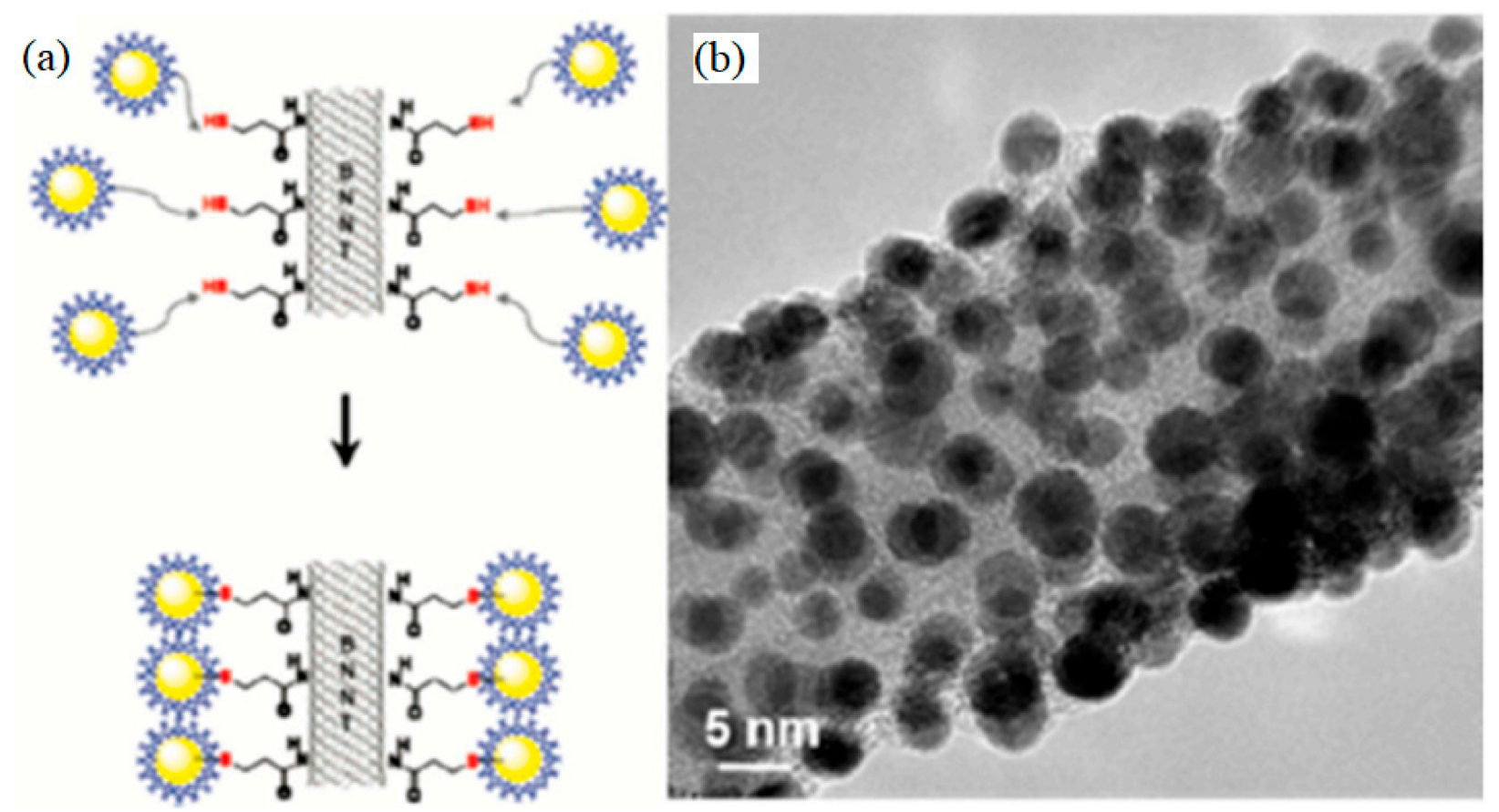

Figure 8. (a) Schematic representation of the synthesis, by Au NPs, of the NTs of Thiol pendant groups functioning decorative boron nitrid, (b) the relevant TEM picture, reproduced with a reference authorization, ACS, 2004 [78].

(a)

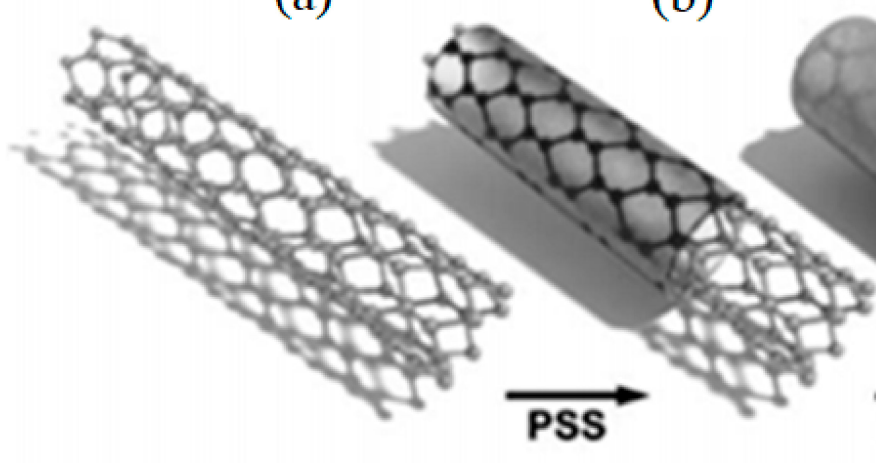

(c)

(d)

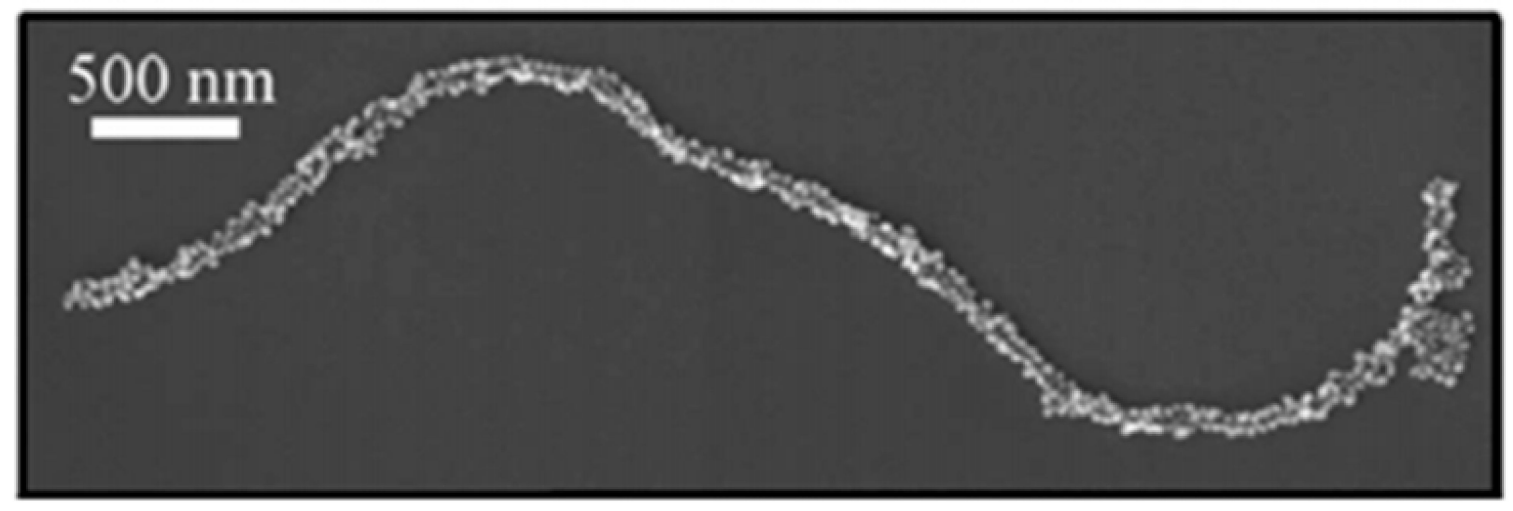

Figure 9. Diagrams illustrating a non-covalent CNT functionality consisting of (a) polymers wrapping by poly(4-styrene sodium sulfonate) (PSS), (b) poly(diallyldimethylammone chloride) (PDDA) self-assembly, (c) nanoparticle deposition and (d) SEM (top) image of one $\mathrm{Au} @ \mathrm{SiO}_{2}$-nano-particles monolayer, assembled in the reference carbon nanoparticles, RSC, 2006 [81]. 


\subsubsection{Polyoxometalate Compounds (POM)}

The POM species are in the colloidal, molecular and even smaller ranges than the Au $\mathrm{NP}$, as established a full class of nano construction blocks by Moor W [85]. The mixture of numerous improved characteristics, and their capacity to act as completely oxidized photoreducible compound. Because of their anionic charge, chiefassociationsamong POMs and the metallic NPs are accompanied by the electrostatic connections. In this way, the POMs act as the protective ligand shell part by surrounding the metallic nanoparticles [86-89]. The improvements of POM products for organic inorganic blends made the covalent POMs adjacent to metallic NP alternative categories. These organic inorganic hybrid POMs from a lacune POM are intended to contain a surface of more nucleophilic oxides, with organosylla groups ( $\mathrm{RSi}(\mathrm{OR})_{3}$ type), which can confirm W-O-Si covalent interconnection [90]. By this method Au NP are covalently encircling hybrid POM, Mayer et al. [91] used this hybrid POM for pouring into Thiol groups. The link between nanoparticles and POMs was confirmed by the use of mercaptoorganosilyl group [86]. Shweta et al. [91,92] described other analogous improvements later. Many methods were defined for the modification of POMs including the functioning by organo-amino groups of the POM core laid down in 2019 by the Leroy group [93]. POMs can be used as reduction and coating agents in the designation of Au nanocomposites. Alternative methods involve the use of a reduced polyoxovanadate with biphosphonate molecules acceptable to synthesize organic-inorganic composites coated with Au nanoparticles in a single step. The novel nanocomposites were introduced to strongly prevent P. aeruginosa and S growth. Biofilm Epidermid (see Figure 10) [94].

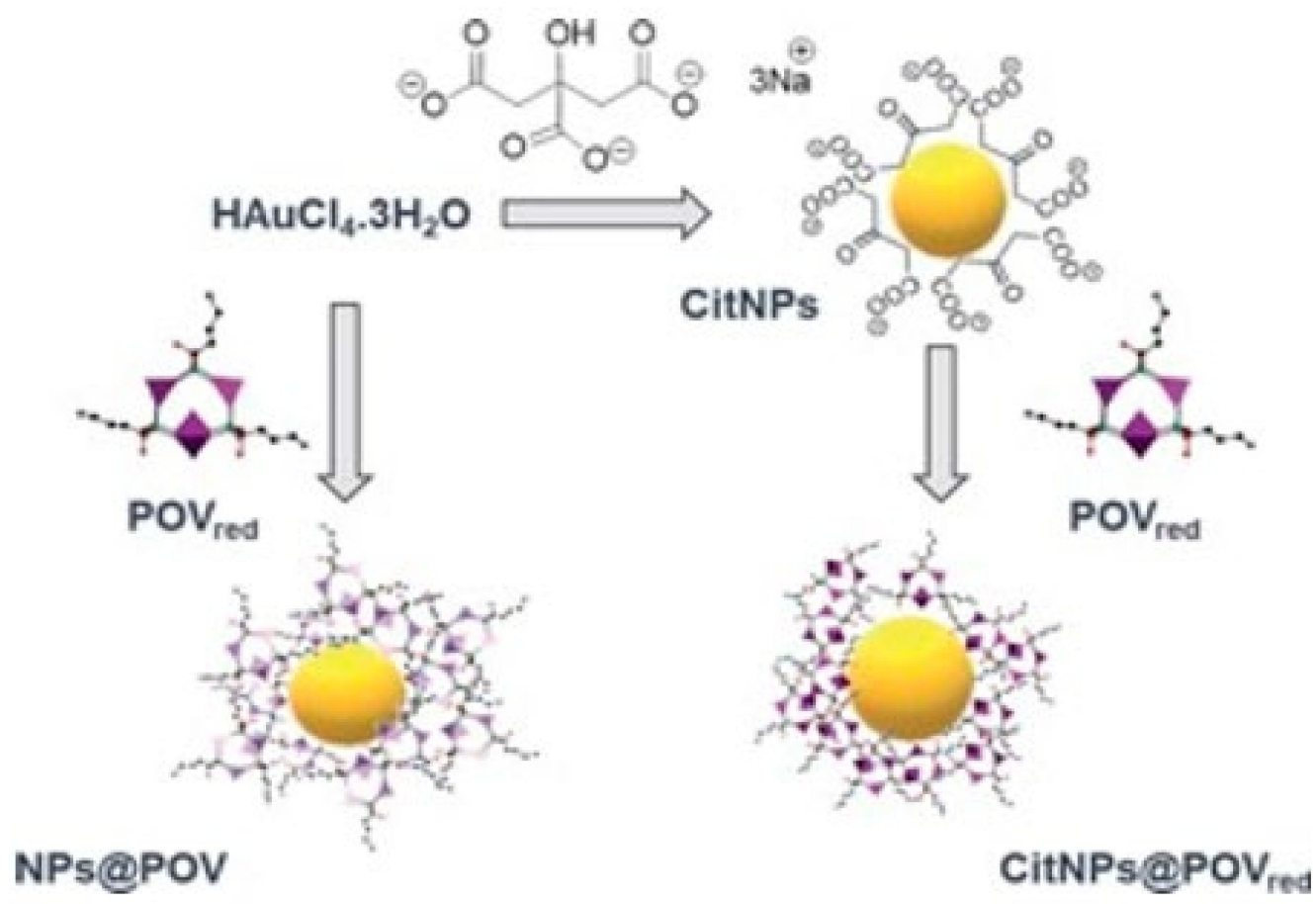

Figure 10. Schematic representation of CitNPs, CitNPs@POVred and NPs@POV synthesis, reprinted with consent from reference [94], RSC, 2019.

\subsection{Functionalization via Organometallic Complexes}

The functional use of Au NPs through organometallic (ON) complexes, mainly due to its electronic characteristics in redox-based sensors, is consistent through ferrocene complexes. The other ruthenium-based OMs as metals were also implanted on Au NPs as summarized by Wilton-Ely for catalysis [95]. Diverse OMs complexes reviewed and extended it to metallodendritic complexes. 


\subsubsection{Ferroncenyl Complexes}

This process allowed the development of ferrocene-containing NPs with ferrocene substituted thiols as funtional agents, by functioning Au NPs across multiple groups. Various connectors, such as ferrocenyl hexanethiol, were confirmed for direct preparation of Au NPs (see Figure 11) [96]. It showed a sufficient length linker to ensure that the size monodispersal of NPs is controlled well [97]. Other linkers like aromatic groups, such as the ferrocene thiophenol group, were also employed [98-100].

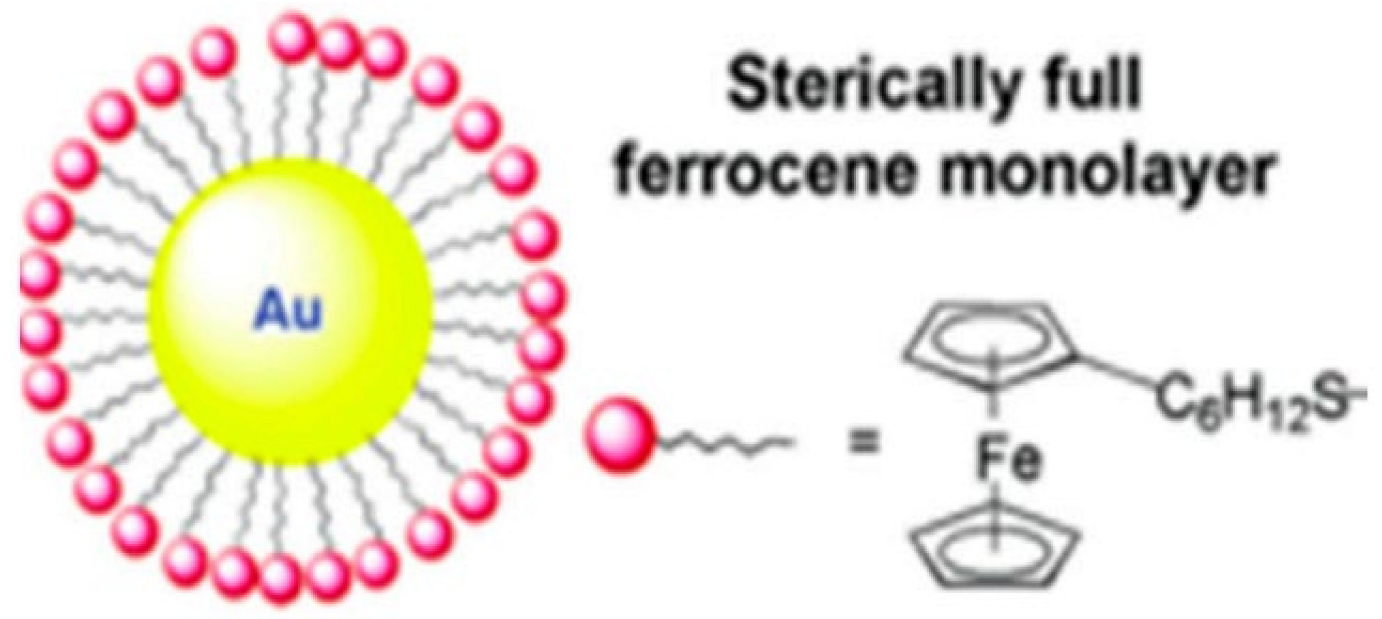

Figure 11. Typical thiophenol ferrocene scheme, replicated by reference authorisation, ACS, 2007 [97].

In ligand exchange reaction, the additional technique to operate Au NPs is used. The heterofunctionalization of Au NPs using this approach is employed by Murray et al. [101] in many functional groups. Firstly, ferrocenyloctanethiol was consequently substituted with diverse alkanethiols. Secondly, by using ferrocenyl octanethiol, the same group formed monospice ferrozen Au-Nps [102]. Another method is recently established by Astruc et al. [103], functionalization containing Au NPs through cross-olefin metathesis. Au NPs are pre-functionalized with olefin-finished groups (methyl acrylate). Then nanocomposites are given through the Grubbs catalyst via the cross metathesis of ferrocenyl methyl acrylate and the olefin replacement Au NPs. Ferrocene-Au NPs are also considered for electrochemical applications. Compared with the first research name, the ferrocene $\mathrm{Au}$ NPs were also synthesized using $\mathrm{H}_{2} \mathrm{PO}_{4}{ }^{-}$and $\mathrm{HSO}_{4}{ }^{-}$anions for redox sensors. Astruc et al. [104] were the first to develop this method in 2000, while using amidoferrocenyl dodecanethiol groups. They measured amidoferrocenated groups remarkably in order to track their appreciation characteristics [105]. The interaction between the amidoferrocene amide group and anion is based on the double hydrogen bond. Figure 12 provides ferrocene NPs for this analysis [106]. Complexes were investigated and biferrocene was the simplest. In order to operate the Au NPs and deposit them, Nishirada et al. [107,108] developed ferrocene-terminated alkanethiols. Alternative study blends ferrocene and bisferrocene with terpyridine ligands to make redox-functionally functional ruthenium(II) [109]. Then Astruc et al. [110] prepared the dendrimers of three amidoferrocene groups or three silylferrocene groups to achieve the appreciation of $\mathrm{H}_{2} \mathrm{PO}_{4}{ }^{-}$anion. On the Au NP surface, the ligand location exchange method was applied to the three ferrocene-based dendrimers. In particular, they extended the procedure to include up to 9 ferrocene-based moieties of larger metallodendron. In this method, they calculated that the ferrocene-dendritic-Au NPs showed 360 ferrocene-based units at the edge of the nanocomposite (for the largest dendrimer). Using these nanocomposites, different anions can be accepted, for example, the well-known adenosine 5 triphosphate [111,112]. 


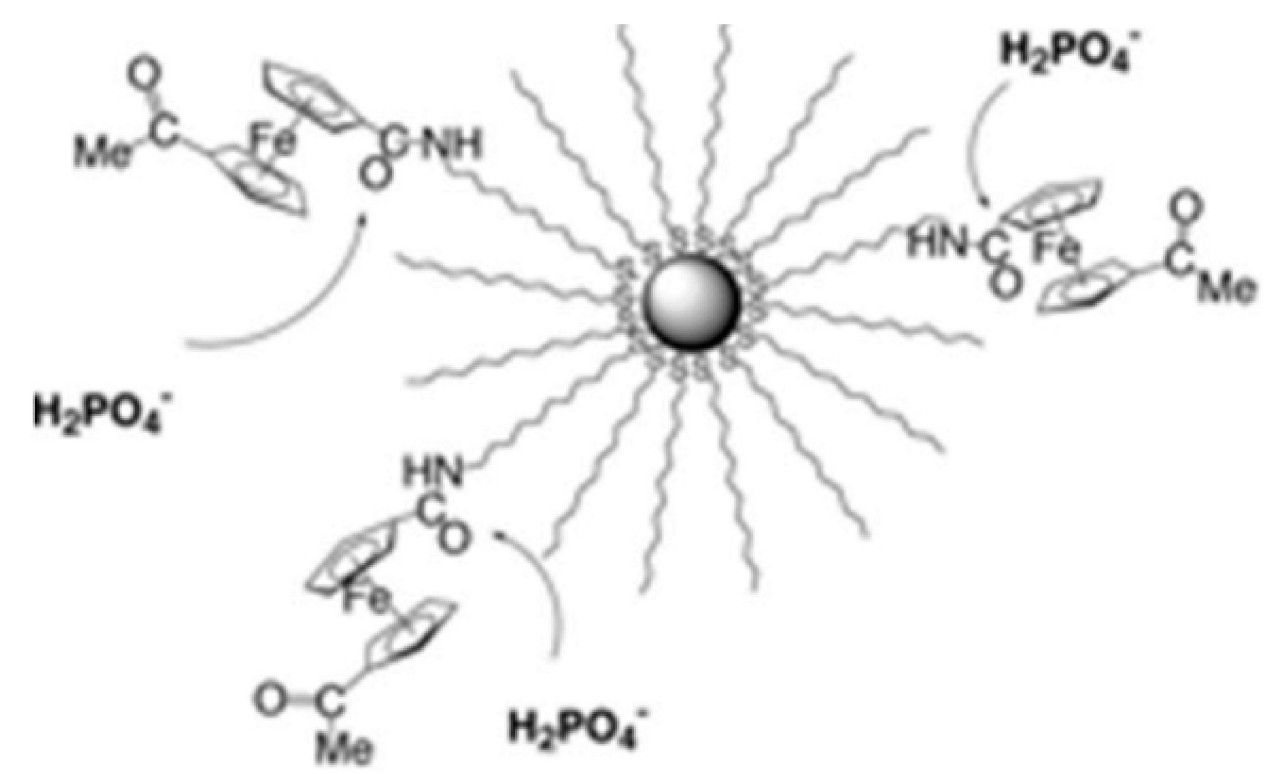

Figure 12. Scheme of Amidoferrocenate $\mathrm{Au} \mathrm{NP}$ and $\mathrm{H}_{2} \mathrm{PO}_{4}$ anions identification, reproduced under reference permission [106], ACS, 2002.

Different polyferrocene complexes have been predicted to advance the redox properties or the feeling in anion sensing. The reuse of organic compounds is an active area of research and Au NPs based on ferrocenyl are used as catalysts for 4-nitrophenol recovery [113]. Recently, advances have been made in the surface functioning with ferrocenyl-Au NPs, for which organic coating distortion has been perceived. Therefore, it is possible to achieve a stable cover or ferrocenyl Au NP adsorption on the metal surface [114].

\subsubsection{Au NPs/Organometallics}

$\mathrm{Au}$ NPs are relatively uncommon functionality by organometal complexes (OM). The tetranuclear complex $\left[\mathrm{Fe}\left(\mathrm{n}_{5}-\mathrm{C}_{5} \mathrm{H}_{5}\right)_{3}\left(\mathrm{n}_{3}-\mathrm{CO}\right)_{4}\left(\mathrm{n}_{5}-\mathrm{C}_{5} \mathrm{H}_{4} \mathrm{CONH}\left(\mathrm{CH}_{2}\right) 11 \mathrm{SH}\right]\left(\mathrm{H}_{2} \mathrm{PO}_{4}{ }^{-}\right)\right.$is relatively similar to the organometal complex in the ground state. The catalytic property of the Palladium-(II) OM complexes was greater. The thiol complex of OM Pd-(II) was created by Fratoddi et al. [115] and the thiol function has been connected directly to the center of the Pd-(II). The composite material is synthesized through a direct functionalization process (Brust's process). The $\mathrm{Ru}$ (III or II) or Rh (I) OM preparation was carried out for catalysis [116] to ensure good fixation of OM on the surface of the Au NP. The ruthenium complexes with two or four alkyl thiol side groups were fabricated $[117,118]$. The OM complex of rhodium is mononuclear and bound to an Au NP by amidododecanethiol (see Figure 13) [118].

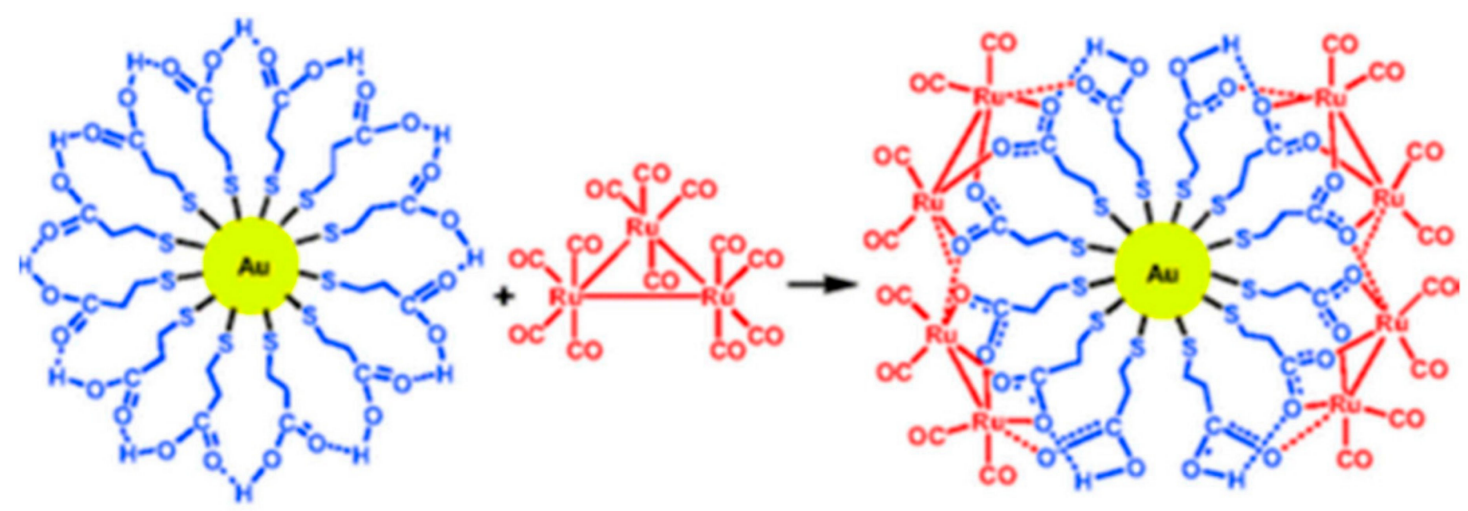

Figure 13. Scheme of Au NPs functioning through trinuclear ruthenium complexes, replicated with reference authorization [118], ACS, 2006. 


\subsection{Functionalization of d-block Element Coordinating Complexes}

\subsubsection{Prussian Blue Derivatives}

The combination of several Nanocomposites successively linked to Prussian blue products by Au NPs has been identified. Different methods were putforwarded for the preparation of nanocomposites. In a single step process, Au NPs and Prussian blue (PB) composite was produced electrochemically [119]. The practical electrode was positively used to catalyze $\mathrm{H}_{2} \mathrm{O}_{2}$ reduction and nanomolar sensitivity in its amperometric recognition. PB@Au nanoparticles were obtained with diameter in the range of 20 to $50 \mathrm{~nm}$ (see Figure 14). Through possible cycling electrodeposition, formerly, the similar type of functionalization with Au NPs stabilized with dendrimers (PAMAM: polyamidoamine) was done [120]. Particles less than $3 \mathrm{~nm}$ were present. A chemical method can also be used to synthesize PB@Au nanocomposites [121]. Consequently, in the presence of $\mathrm{Fe}(\mathrm{CN})_{6}$ PB-functionalized Au NPs of an average size of $50 \mathrm{~nm}$ are provided by reductions in ferric ions in water The Langmuir-Blodgett method developed the PB@Au-multilayer thin films into an $\mathrm{H}_{2} \mathrm{O}_{2}$. In both processes (chemical and electrochemical), the functionalization of particles was performed by an electrostatic bonding between Au NPs and PB.

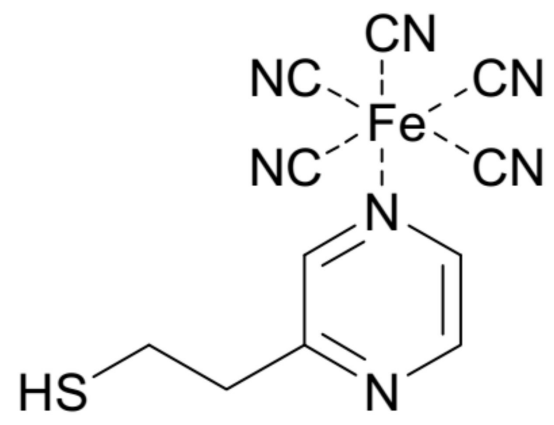

$\left[\mathrm{Fe}(\mathrm{CN})_{5} \mathrm{PZT}\right]^{3-}$<smiles></smiles>

\section{$\left[\mathrm{Fe}(\mathrm{CN})_{5} \mathrm{~L}\right]^{3-}$}

\section{with $L$ : 2 or 4-mercaptopyridine}

Like 2-pyrazin-2-ylethanethiole [123] or 2 and 4-mercaptopyridine [124], the functionality of the metal complexes on Au-NTs can be defined. In the last case, the binding of the particles can be felt by using 2-mercaptopyridine, but stable particles can be obtained with 4-mercaptopyridine or 2 and 4-mercaptopyridine.

\subsubsection{Metal Complexes of Polypyridyls}

The bidirectional ligands assisted complexes are commonly used as inorganic molecular entities (IMEs) for NPs of Au. Polypyridyls of the metal complexes can be used by electrostatic interaction or through an adhesive group on Au NPs. The facial application of $\mathrm{Au} \mathrm{NPs}$ is an amazing research field. It is a complex option to combine tris(bipyridine)ruthenium(II) of the various current metal complexes mixing light and electrochemical possessions. The Au NPs can be functionalized by two separate methods via bipyridine complexes. The first is that metal complexes are directly interacted through electrostatic interactions with particles. The unmodified tris(bipyridine)ruthenium(II) complex is directly implanted by several books. Its fundamental features establish an appropriate analysis in which the appropriate transfer of energy, electron transmission or higher rate of crossing between the Au NPs and the complex is to be evaluated. Murray et al. [125] mainly described luminescent squandering of Ru(bipy $)_{3}{ }^{2+}$ in Au NPs, with metal surfaces recognized to be able to squeeze excited molecular states in a way that (see Figure 15). 


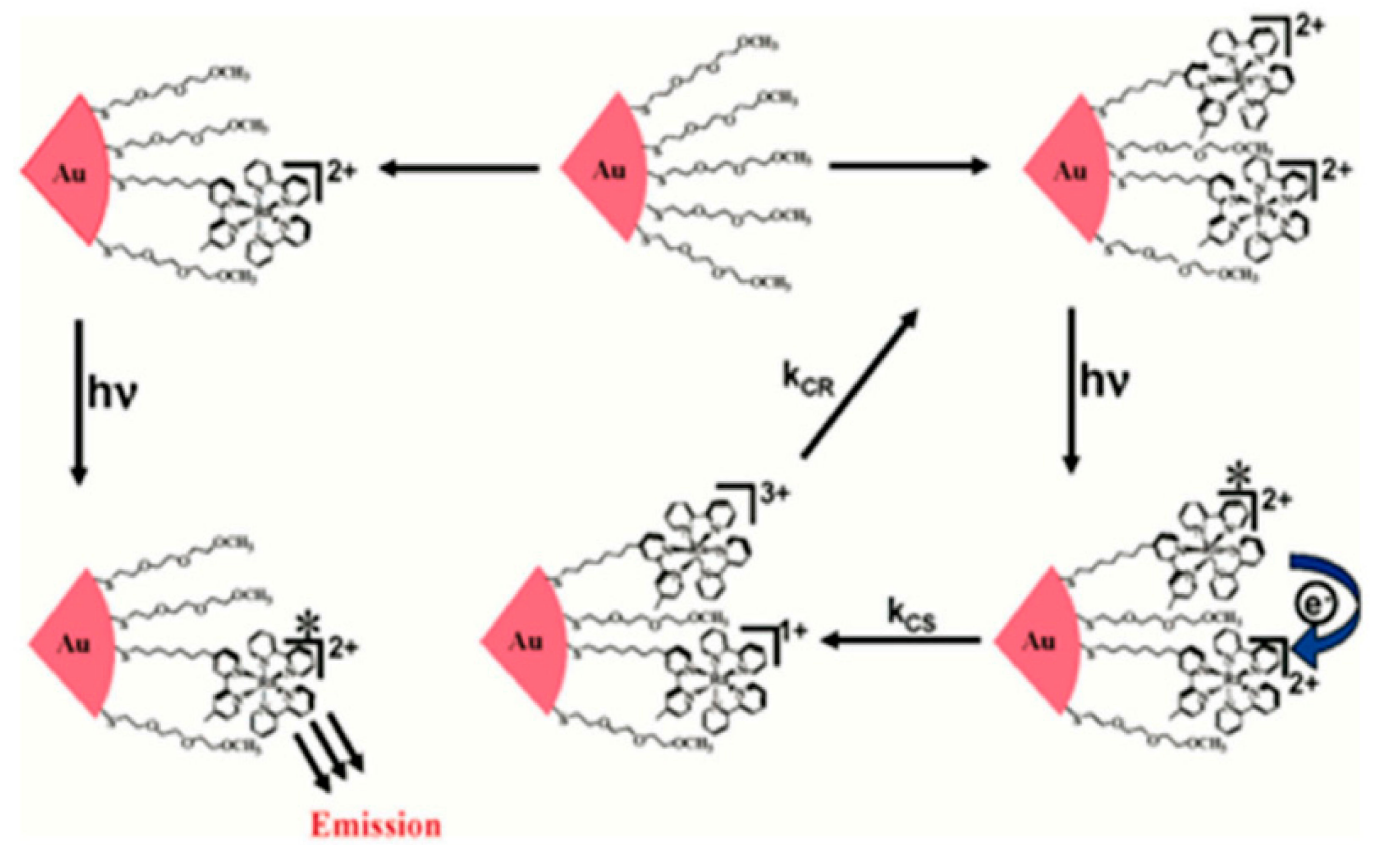

Figure 15. Tris(Bipyridine) assisted the Ru (II) light-inducing process complex of Au NPs, replicated with reference permission, ACS, 2006, within this Au-(S-C7-Ru) method [125].

Fluorescence quenching with tiopronin-protected Au NPs of different diameter was examined. A pure rise in extinguishing proficiency with core diameter was demonstrated, Profitable quantification would most likely involve a reversible electrostatic interaction between fluorophores and particles. Through introduction of $\mathrm{KCl}$ electrolyte in the solution leading to the modification of electrostatic bondings between complex and NPs, The electrolytes are beating the tiopronin carboxylate bonding sites. Alternatively, two opposite stabilized particles using one photon counting spectroscopy were analyzed on the surface area of two adsorbed complexes [126]. In addition to the size and temperature for understanding the luminescence-quenching of Au NPs, the kinetics study of complex adsorption was also examined [127]. Further required factors in Au NPs or nanorods by Au-S bonding have been explored for immobilized Ru(bipy) ${ }_{3}{ }^{2+}$ complex. These factors comprise chromophores, optical and surface reliance on density, size or temperature $[128,129]$. The previous reports aimed to achieve energy or electronic communication among particles and the complex for future applications such as catalysis, biology, optics or electronics. $\mathrm{The} \mathrm{Ru}(\mathrm{bpy})_{3}{ }^{2+}$ complex has also been extensively investigated for its electrochemical lighting (ECL). By means of this stuff, numerous modified electrodes were synthesized for discoveringsolid-state ECL in capillary electrophoresis [130]. In order to improve ECL identification of the indium tin oxide (ITO) electrode, $\mathrm{Ru}(\mathrm{bpy}) 3_{3}{ }^{2+} \mathrm{Au}$ NP collections were synthed and prevented in conductive support by Au-S bondage. The (ITO) electrode, the $\mathrm{Ru}(\mathrm{bpy})_{3}{ }^{2+} \mathrm{Au}$ NP groups have been grouped and limited by Au-S binding on conductive support. These schemes showed massive enhancement of ECL strength, increases the detection and makes it 104 times more sensitive than the deprived imbedded Au NPs. The selective finding of bio-chemical molecules like pentoxyverine were also employed by electrodes modification [131]. More mixed composites were synthesized employing polypyridinyl complexes formed of $\mathrm{Ru}(\mathrm{bpy})_{3}{ }^{2+}$ complex. The ITO electrosse was made from a complex with three thiol pendant groups, with self-assembled layers of the ruthenium complexes [132]. A well-organized 3D stable structure was thus detected at the electrode surface and obvious enhancement of the photocurrent feedback with the number of covers was demonstrated. In dye-sensitized ruthenium(II) solar cells, creation of such $3 \mathrm{D}$-self assemblies could be appreciated. An electroactive spacer like viologen group can be used for the modification of electrodes (see Figure 16) [133]. A 15 times greater sensitivity for the electrode was observed for the anodic photocurrent detection by doping of 
functionalized Au NPs onto Au electrode. A contribution of the viologen entity through one-electron reduction approach showed extension of that early work comprised to know the effect of the size of nanoparticles on the photocurrent reaction.

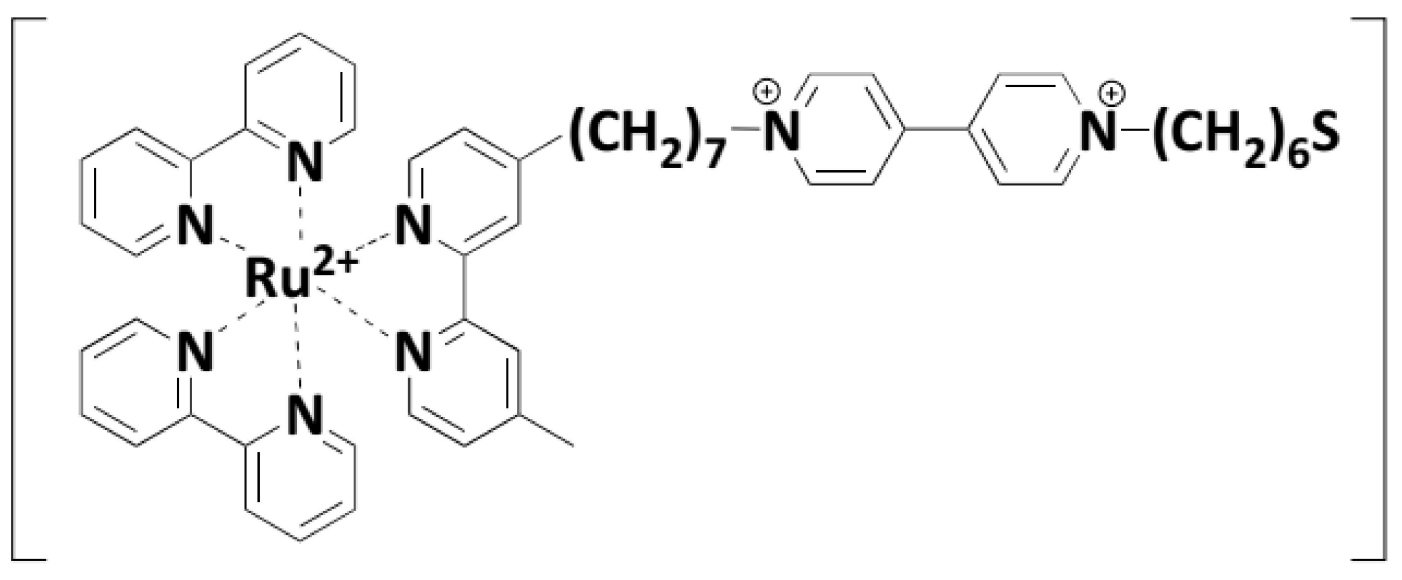

Figure 16. Viologen linked thiol-Ru complex imbeded onto Au NPs, Reprinted with permission from reference [133], Elsevier, 2003.

The perfect photocurrent competences of nanostructured particles have diameters of 50-100 nm [108]. On the basis of this, enhancement of the ionic strength is identified to clumping of Au NPs. In a novel approach viologen-doped ruthenium complex was used for the decoration for these nanostructures [134]. In the said work, the impact of the electrolyte nature, anionic agent in particular was studied because it can cause alteration in the morphology of these schemes and as a result change the photocurrent reactions. In addition to these violin-connected systems connected to Au NPs by a thiol movement, many electrostatic violin systems between $\mathrm{Ru}(\text { bipy })_{3}{ }^{2+}$ and nanoparticles capsized with citrate were inspected $[135,136]$.

No photo-electrochemical cells were obtained and therefore the Au NPs were transferred to the violin sensitizer with energy. The association of Au NPs can also be simply acquired by employing the metalation with pyridine entities [137]. By employing this scheme, the thin films were produced and they showed diode like reactions. For the functionality and stability of Au NPs, some additional anchoring groups have been employed around the redox centre with a polypyridinic environment. Recent work has clarified the effect by the amine end group assisted by $\mathrm{Ru}(\mathrm{bipy}){ }_{3}{ }^{2+}$ complex on the rates of radiation and the non-radiative rates of a phosphorescent compound [138]. A recognition teachniq has been used for implanting phosphorescent molecules on the particles. In case of Streptavidin with a surplus bovine serum albumin, nanoparticles were first synthesized. Phosphorescent molecules were thus functionalized with biotin due to selective sensing of streptavidin for biotin. Hence, anchoring of these NPs- molecules was recognised, and grounded on the biotin-streptavidin sensing aprocah. Biotin-streptavidin appreciation, was another examples which was innovative for the gathering of proteins and Au NPs on templates of DNA [139]. Other characteristics, i.e., of a cobalt bistable complex supramolecular control of valence-tautomeric symmetrywas also studied [140]. The anchoring effect was seen on thermodynamic factors; the binding of the valence tautomer influenced the surface containment. Up to now, only nanocomposites have been identified which were obtained through electrostatic interations or by using aliphatic chains with thiol end groups. No electronic contacts via the non-conjugated spacer were possible in these arrangements. In recent times, Mayer et al. [141,142] reported the preparation of numerous poly-pyridinic complexes of rutheniumof bidentate ligands phenanthroline ligands with completely delocalized insertions, allowing a straight message between the complex and particles (see Figure 17). Ruthenium complexes' redox potential has been changed to display an electronic message. 

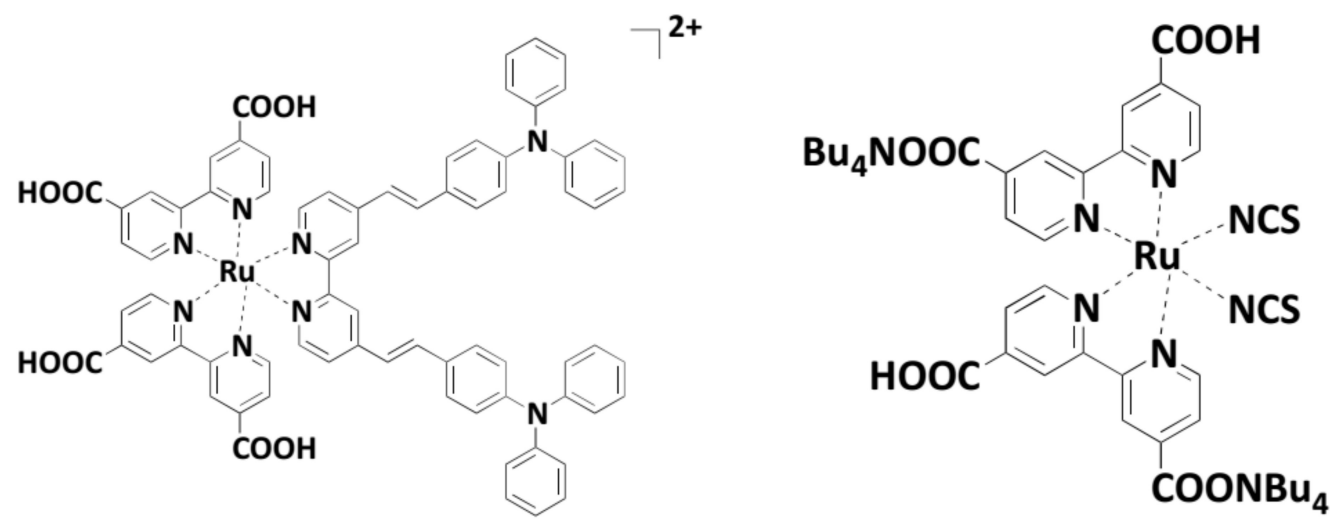

Figure 17. Bipyridyruthenium(II) complexes found in an Au NP via an amino or isothiocyanates group. Duplicates with reference agreement [143], ACS, 2005.

Bipyridyl compounds joint linkers for surface improved Raman dispersion measurements have also been investigated [143]. In particular, the effect of the solvent was examined and several behaviors of difficult adsorption were verified by the findings. The ruthenium complex used in the current study was achieved by inserting a conjugated triphenylamine spacer in a ruthenium chelated core using three bipyridine ligands (temporarily as a pendant) or by adding carboxyllates. Functional agents in all previous examples offered only one anchoring point. However, two binding groups may also occur. This is why two thioline mouths liked a complex synthesized with phenanthroline [144].

\subsection{Functionalizated Coordinated Complexes on Au NPs for Numerous Applications}

It was agreed to use ruthenium complexes to form nanocomposite junctions between electrodes. The nanocomposite is synthesized by means of sulfur reaction and is inserted in two micrometer-holes opposite Au electrodes. The measurements of conductivity were checked and confirmed the effectiveness of this electrical self-assembly, which is due to the presence of ruthenium cations, allowing for enhanced conductivity and poor energy barriers. For other useful applications, bisoxazoline bidentate ligands are used to synthesize complexes that are admirable in catalysis. Therefore, copper-dioxazine chiral complexes with covalently bond to Au colloids have been used in the enantioselective ene reaction of 2-phenylpropene and ethyl glyoxylate [145]. The use of this primitive homogenous catalyst leads to an exceptional profitability and high residue. The catalyst also benefits from the simple detachment of the reaction from the mixture by filtering. Supporting Au NPs is used for other applications for zinc phthalocyanine(II) (see Figure 18) [146].

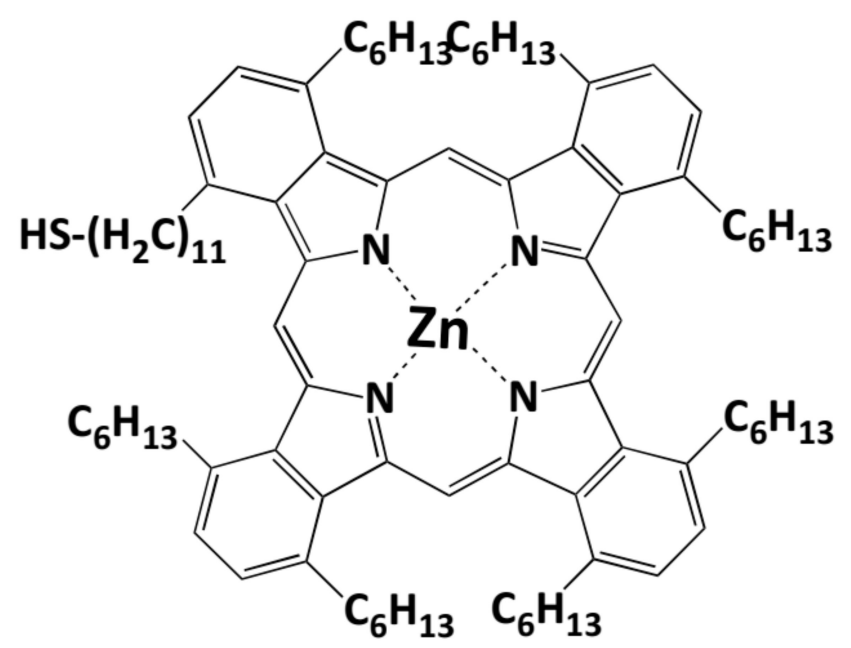

Figure 18. Phthalocyanin Zinc (II) complex for photodynamic treatment coating Au NPs. Reproduced by reference permission [146], ACS, 2002. 
By grafting photosensitizers onto Au NPs, compared to free photosensitizers, the production of singlet oxygen has an increased quantum yield. Such a system can potentially be used to transfer photodynamic photosensitizers in photodynamic treatment. An example of anion sensor of porphyrin linked was lately described in the literature [147]. The grafting to NPs was identified by using four side groups generated from sulfuric acid. Compared with free metalloporphyrins, nanocomposites verified by six different anions show an important increase in anion binding attraction. Corrected the anion attraction caused by the pre-organization of porphyrin on the particle surface. A modified electrode was also synthesized with porphyrin. The layer-by-layer deposition method based on electron acceptors can organize Au NPs that are covalently connected to ITO electrodes. The inspection of these electrodes in the photochemical experiment makes the resulting photoelectrochemical cell less proficiency with this photosensitize. In 2007, Ozawa et al. [148] described the synthesis of porphyrin filaments and the association of $\mathrm{Au}$ NPs with porphyrin $\pi$-conjugated filaments. First, the porphyrin polymer was deposited on the surface of the functionalized glass by Langmuir-Blodgett technology. Later, the glass substrate was soaked by particles of terminated 4-pyridine-ethanethiol, and the particles were grafted onto the glass substrate through the interaction of porphyrin with the pyridine NPs (see Figure 19). This is interesting because the adhesive Au NPs can only be felt when the polymer was already placed on the glass substrate. Biomolecules are related to Au NPs as the final porphyrin-connected device. The porphyrin-causing structure hemichloride (Hem) and cytochromec (Cytc) were administered to NPs and the azide anion was detected [149]. Thermal stimulation must be performed to bind the azide anion due to the reduced accessibility of biomolecule grafting.

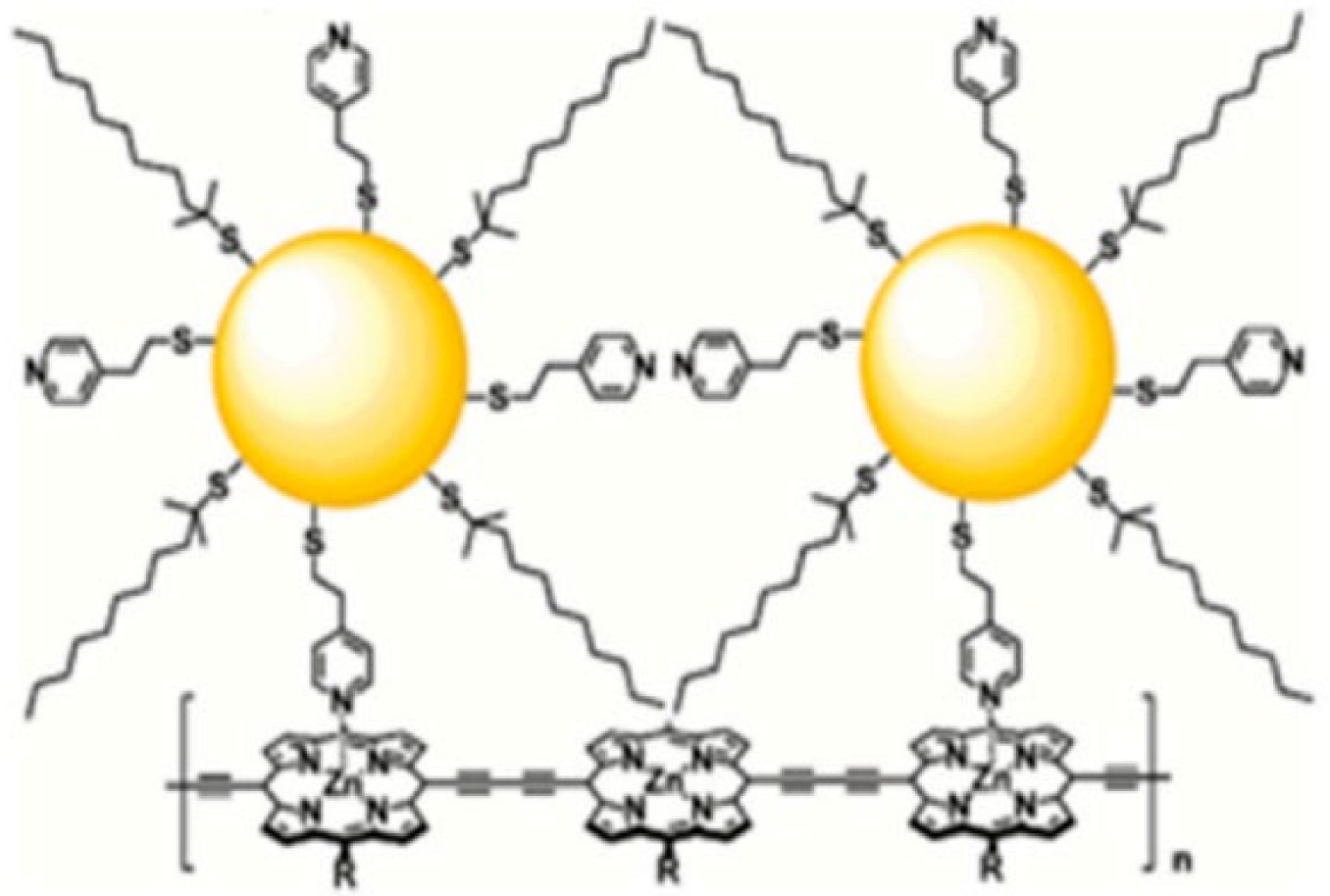

Figure 19. 1D- Au NP chemically connected to porphyrine conjugated, reproduced by reference permission, ACS, 2007 [148].

\subsection{Functionalization via Shiff Base Coordinated and Carboxylates Linked Complexes}

Many charged ligands complexes have also been aplied to coat Au NPs in a singlelayer organo-metallic complex shell [150]. Therefore, the coordination of metal ions can be understood by the carboxylate functional group. The focus on such complexes obscures coordination, which may be destroyed "on demand" only through stronger chelating agents that separate the complexes. An important application of this system is the discovery of ions of heavy metal [151,152], due to the accumulation of the nanoparticles in the incidence 
of cations, the synthesis of self-assembled monolayer [153-156], or nanocomposites additionally appreciated in catalytic schemes [157]. The first two uses, i.e., nanoparticles, were appropriately functionalized by mercapto-alkyl acid, the carboxylate group acting as the pendant group enabling to create interparticle forces below metal ion chelation (see Figure 20).

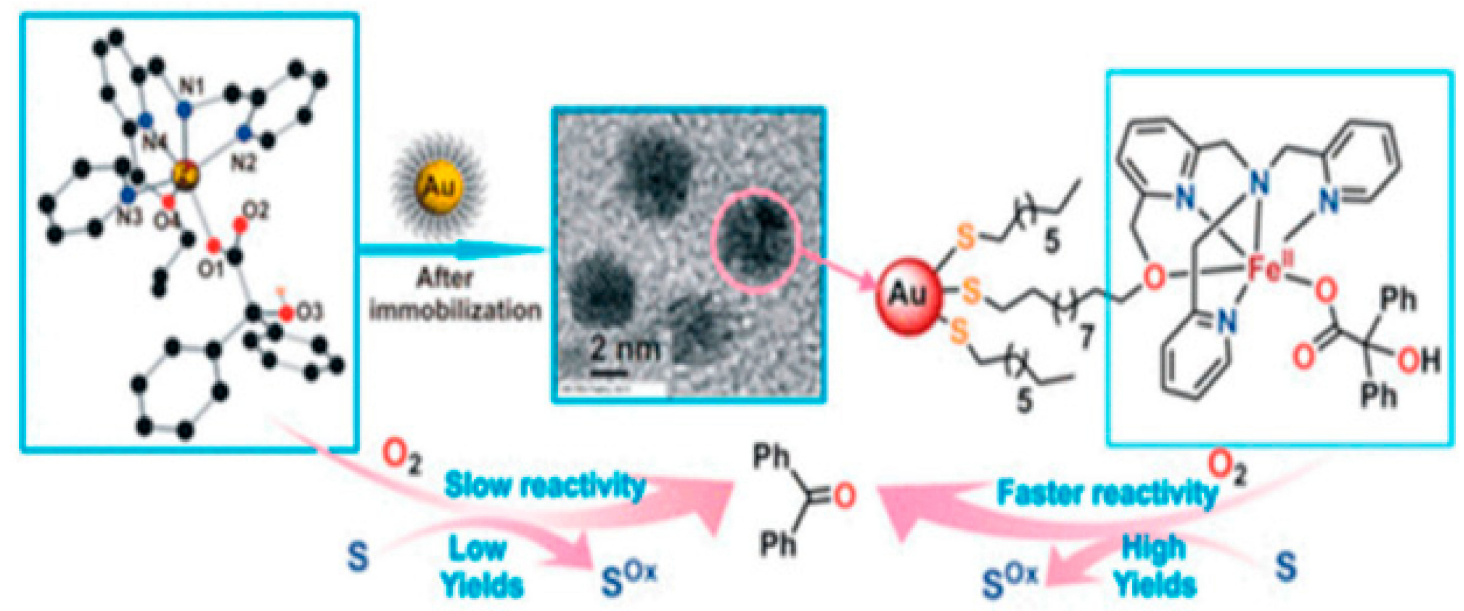

Figure 20. Description of the ability of oxidizing agent of a dioxygen-activating from complex of non heme Iron(II)-Benzilate immobilized on Au NPs, reproduced with permission from reference [150], ACS, 2019.

The Ru dodecenyl ligand complex develops a multi-stage synthetic method for the final usage; the carboxy group reacts to the complex to synthesize the Ru carboxylate carbonyl complex (see Figure 21). The dimer or oligomer was bound to the particles by this method. Additionally, the metal complex may be integrated into the particulates with other functions. Thus, bis-Hydroxamate ligands were used to create monolayers or multilayer coordinated $\mathrm{Zr}^{4+}$ nanopartments [158]. A bifunction molecule (gallic acid) with one group of carboxylates as capping agents and a ligand were used to prepare the naked eye detector for a co-ordinated $\mathrm{Pb}$ (II) cation with a group of hydroxyl. Since, the $\mathrm{Pb}$ (II) cation has a special coordinating behavior, its coordination number can be extended to twelve, thus leading to an aggregated $\mathrm{Pb}(\mathrm{II})$ cation.
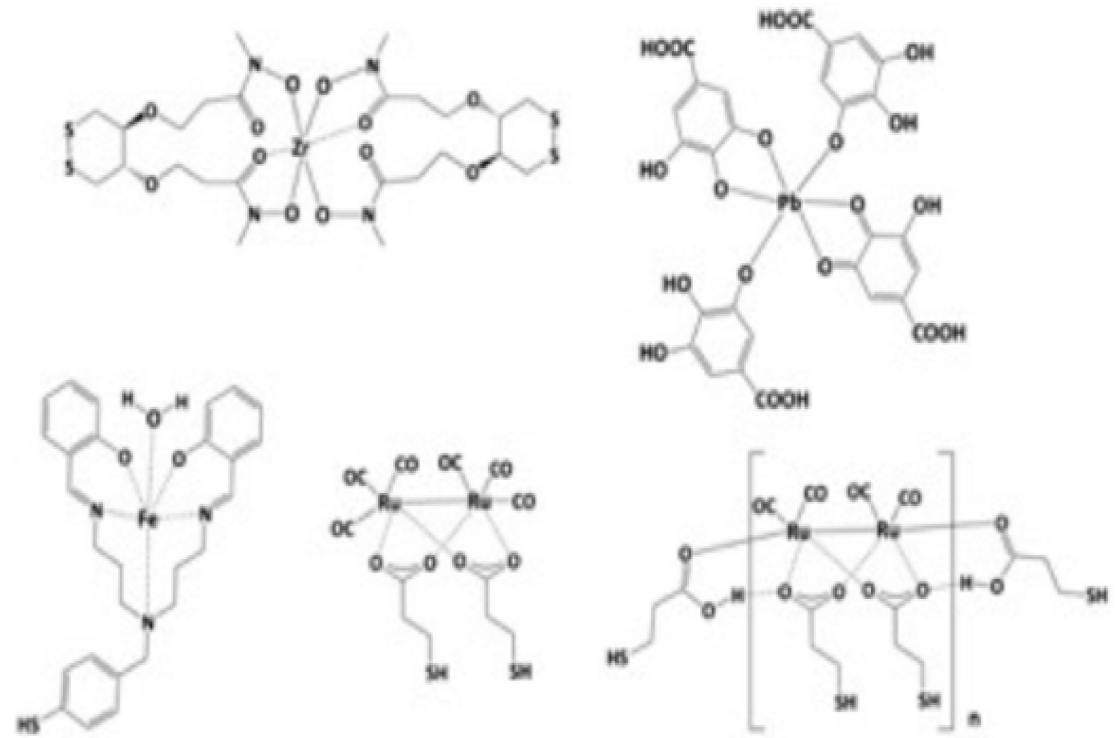

Figure 21. The example of combined complexes used in Au NP coat is carboxylate, amidate and phenolate. Reproduced with permission from reference [158], ACS, 2005. 
The introduction of different metal cations leaves NPs remote, since they co-ordinate to overcrowded electrostatic disintegrations between particles with less ligands and the ability of cation $\mathrm{Pb}$ (II). For the coordination of iron (III) cations, the Schiff base ligands were used. For the stabilization of Au-Nps, two opposite plans were used in that work. Firstly, using the neutral complexes for the stabilization of the NPs through steric (alkyl chain) repulsion and secondly the stabilization is gained through electrostatic repulsion [159].

\subsection{Functionalization via Bio-Inorganic Complexes}

The last kinds of ligands capable to bind with metal ions are wholly ligands supported on biomolecules. Because of their uses in electronic, optical and biosensors, increasing attention has been given to the nanocomposites modified with biomolecules [160,161]. The Co (II) complex was formed due to the covantly binding of proteins with Au NPs. Genetic engineering protein enables a histidine chip that is able to connect to the $\mathrm{Co}$ (II) ions via ligand exchange on a Co(II) complex imbedded (see Figure 22) [162]. Numerous illustrations of glycol nanoparticles have been synthesized to study forces of carbohydrate with cation of $\mathrm{Ca}$ (II) in water [163]. In the aggregation of particles of calcium cation-carboncarbohydrate contacts, $\mathrm{Ca}$ (II) ion complexation is caused. The introduction of strong chelator like EDTA and reversible forces brings about the redispersion of the particles. The effect of the spacer length on complexation was also investigated andthe shortest linker gives bottom-finding level.

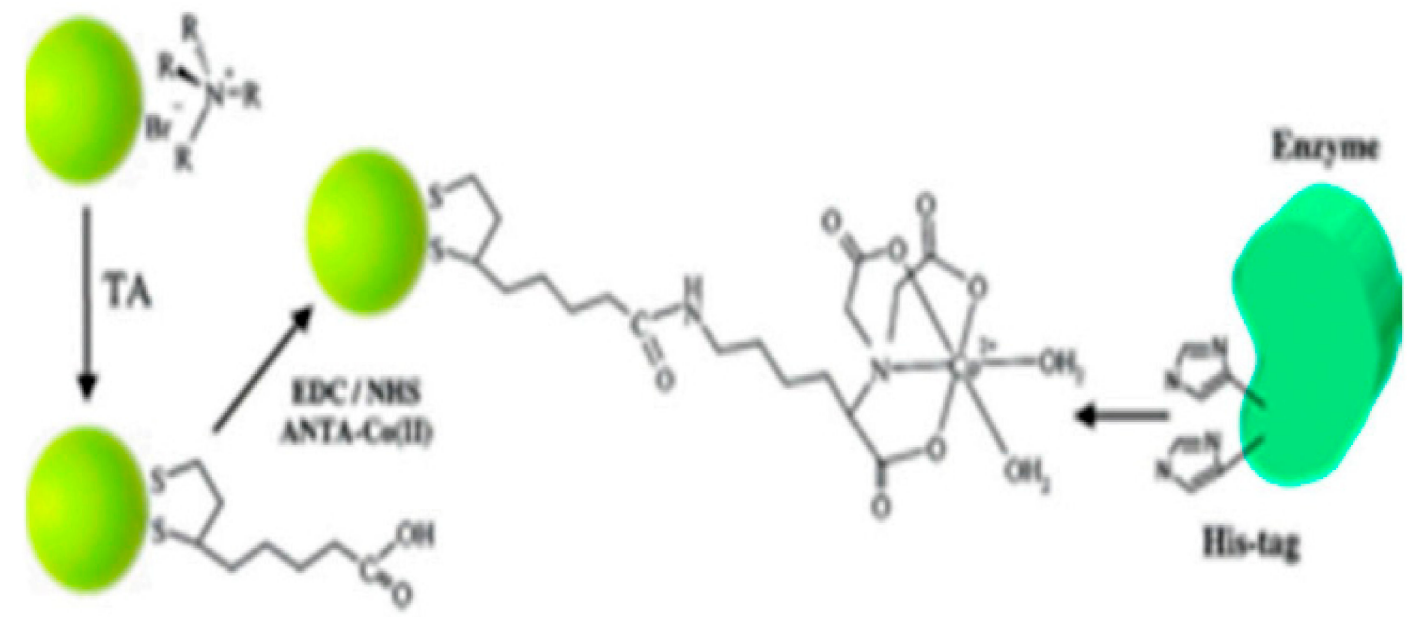

Figure 22. By immobilizing completely functioning proteins on the Au NP surface, biomolecules are grafted on Au NPs by the use of thioctic acid. reproduced with reference permission [162], ACS, 2005.

For the finding of $\mathrm{Hg}$ (II) ion Au NPNPsm peptide functionalized nanoparticles have also been synthesized [164]. Peptide functionalized Au NPs showed amino and a carboxylic group at both ends first time. Through the amino group, attachement of the peptide was performed. Beause of the strong attractions towords amino groups by $\mathrm{Hg}$ (II) cations, peptide from the surface of the particle was separated due to cation's introduction. A colloidal particle 1D linear assembly was observed. An EDTA alkali solution has been introduced to isolate peptide from the $\mathrm{Hg}$ (II) ion and to engross open peptide on the surface. $\mathrm{Au}$ NPs, with DNA-functionalization, have also been usedfor the colorimetric recognition of numerous cations like $\mathrm{Hg}$ (II) [165], $\mathrm{Pb}$ (II), Cu (II) [131,166-169]. The complex of ruthenium behaving as both DNA hosting unit and the linker was used for the coating of DNA to Au NPs [141]. The incorporation of the DNA template was performed by first preparing the streptavidin-coated Au NPs and the complex of ruthenium was added in suchaway that coordination of streptavidin and phenazine ligand to biotin-phenanthroline ligand capable of being poured into a duplex of DNA was observed (see Figure 23). 


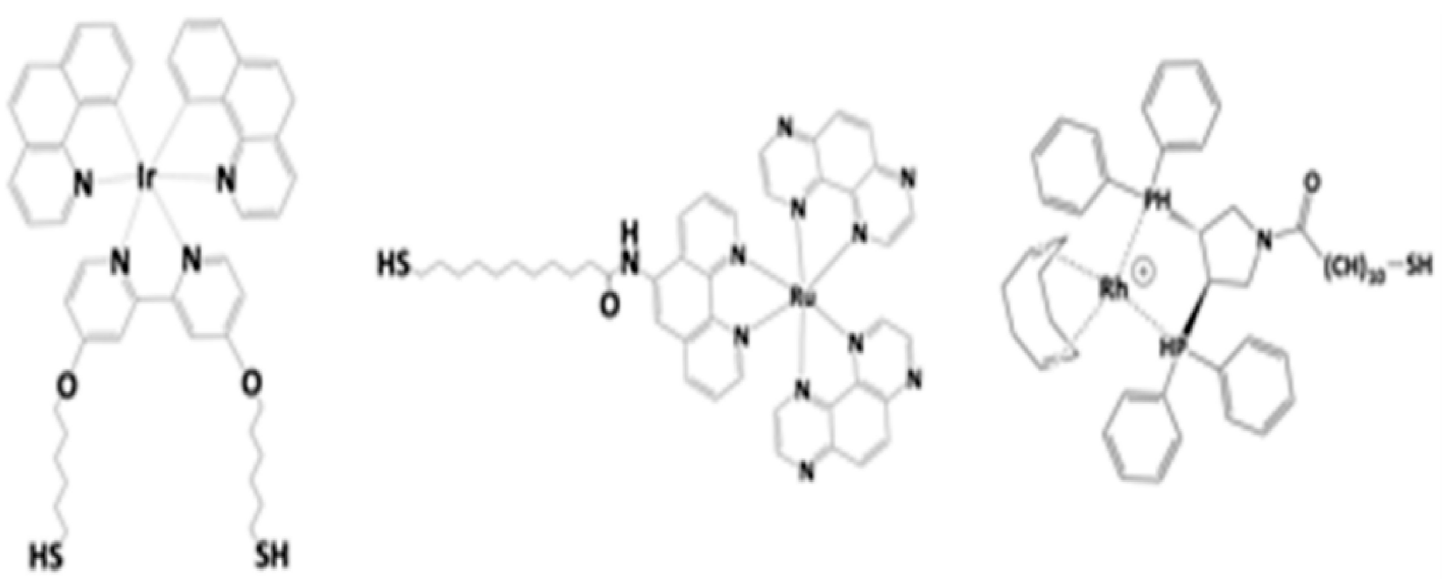

Figure 23. For cell-level imagery used for the surrounding Au NPs, catalysts or metallic complexes. Reproduced with permission from reference [141], Nanomaterials, MDPI.

For the structural and catalytic studies, numerous covalently bonded metal complexes were applied to Au NPs. For the enantio selective hydrogenation of acetamidocinnamate, the chiral complexes of rhodium-diphosphine on Au colloids were employed [146]. Binolate Particle Ti-complexes were synthesised and added to the asymmetric alkylation reaction catalysis of benzaldehyde [170]. The dimeric ruthenium complex was connected to metathesis catalysis particles, such as a polymerisation reaction that opens the ring [113]. Huge nanoparticles supporting ruthenium complexes were applied for imaging of cell luminescence, which discovered their way of biomolecular application linking with cancer cells of chromatin in the nucleus [171]. Nowadays, complexes of ruthenium adjoining $\mathrm{Au}$ NPs are employed for collagen photo crosslink [172].

\subsection{Functionalization via Crown Ether Devices}

For chelation of the etal cations of the d-block elements, crown ethers were employed. Here two illustrations are stated. Initially 2-(12-mercaptododecyloxy) methyl-15crown- 5 was assisted by the Au NPs and monolayer synthesis was applied to hold $\mathrm{Pb}(\mathrm{II})$ cations [173]. The 2.8 to $10-10 \mathrm{~mol}$ per $\mathrm{cm}^{2}$ of $\mathrm{Pb}$ (II) capacity of trapping was attained. Second, the synthesis for $\mathrm{Pb}$ (II) optical sensing was carried out in two dissimilar ligands (2-(12-mercaptododecyl) methyl-15-crown-5 and thiotic acid) embedded in two-functional $\mathrm{Au}$ NPs $[174,175]$ (see Figure 24). The collection of nanoparticles due to interpartum hydrogen bonding was observed without introduction of $\mathrm{Pb}$ (II) cations. The introduction of $\mathrm{Pb}(\mathrm{II})$, a sign of nanoparticular dispersion, by betrayal interpartmental hydrogen bonding, was perceived as an important color shift. There was also a high cation sensitivity for $\mathrm{Pb}(\mathrm{II})$ nano-composite.

The sensing of alkaline metal ions has been of major importance in biology. Amongst the latest aproaches to the design and fabrication of alkali sensors, Au NPs suported sensors have engrossed considerable interst as a capable functional tool. By the introduction of alkali cations, alteration in the intensity of fluorescence or color of the solution is the simplest way of signalling. Crown ethers are used to chelate alkaline cations as crown ethers form stable, alkaline cation complexes. Of all the crown ethers, the 15-crown-5 produces exceptionally stable $\mathrm{Na}^{+}$cation complex, while the 18 -crown- 6 prefer $\mathrm{K}^{+}$(see Figure 25) [176,177]. 


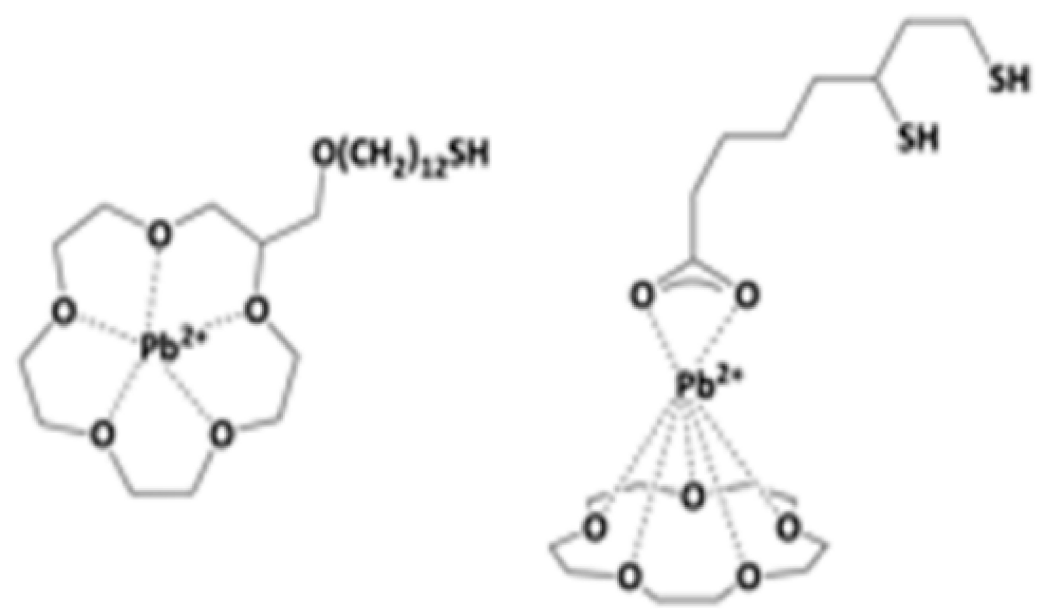

Figure 24. Examples of crown ethers supported onto Au NPs. Reproduced with permission from reference [141], Nanomaterials, MDPI.<smiles>CCCCCCCOCCOCCOCCO</smiles>

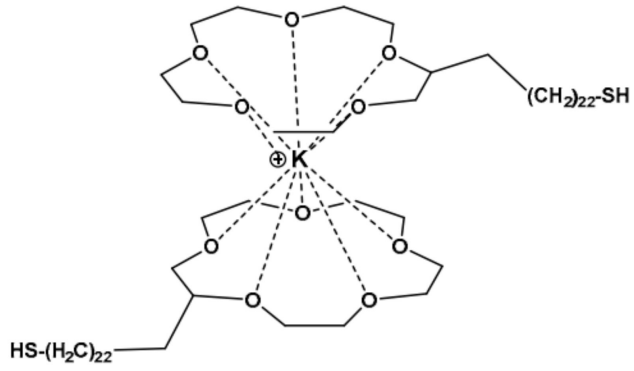

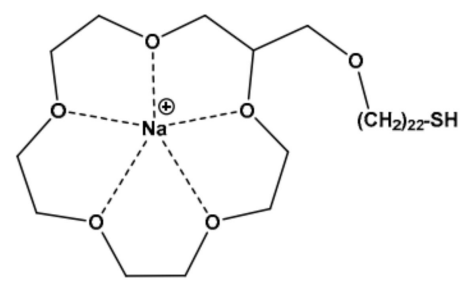<smiles>CCCCCCCCCOCC(COCCO)OCCO</smiles>

Figure 25. Au NPs functionalized via alkali crown ether. Reproduced with permission from references [178], ACS, 1999.

The 15-crown-5-functional Au NPs was first used for colorimetrical sensing, in which a dodecyl chain links the crown ether with the particles [179]. Particles of water stability were obtained due to steric repulsions between particles. The clear change in colour was seen after introduction of $\mathrm{K}^{+}$due to the aggregation of the particles. Two-crown ethers confirmed the stabilization of $\mathrm{K}^{+}$cation. Two different complexations were noticed of the chelation of $\mathrm{K}^{+}$cations with crown ethers. One more group of research has benefitted by synthesizing assembled nanoparticle films due to the addition of cations [180]. The first time, the update was spread by the inclusion of the second nanocomposite feature [181]. When thiotic acid was introduced into the nanoparticles, a supporting effect was observed. Thioctic amine changed its characteristics by the substitution of the thiotic acid. Nanoparticles with bifunctionality were synthesized via a two-step process: firstly, the exchange of ligand in citrate with thiotic acid and, secondly, the unfinished exchange of thiotic ligands through thiolated crown ether [182,183]. The spacer effect on the complexation kinetics was also investigated. A similar investigation was seen with $\mathrm{Na}^{+}$as a cation 12 -crown-4.

\subsection{Functionalization via f-block Elements Coordination Complexes}

Nowadays, numerous works of literature show the functionalization of lanthanide complexes on Au NPs [184,185]. Due to their brightness, several complexes have been 
covalently grafted onto Au NPs to synthesize the ion sensors. In the first case, diethylene triaminepenta acetic acid (H5DTPA) was replaced by two thiope phenol groups to confirm the complex's strong binding to the nanoparticle's surface [186]. Eu (III) nanocomposite and luminescent nanobeads were thus achieved with strong water solubility (see Figure 26) [186].
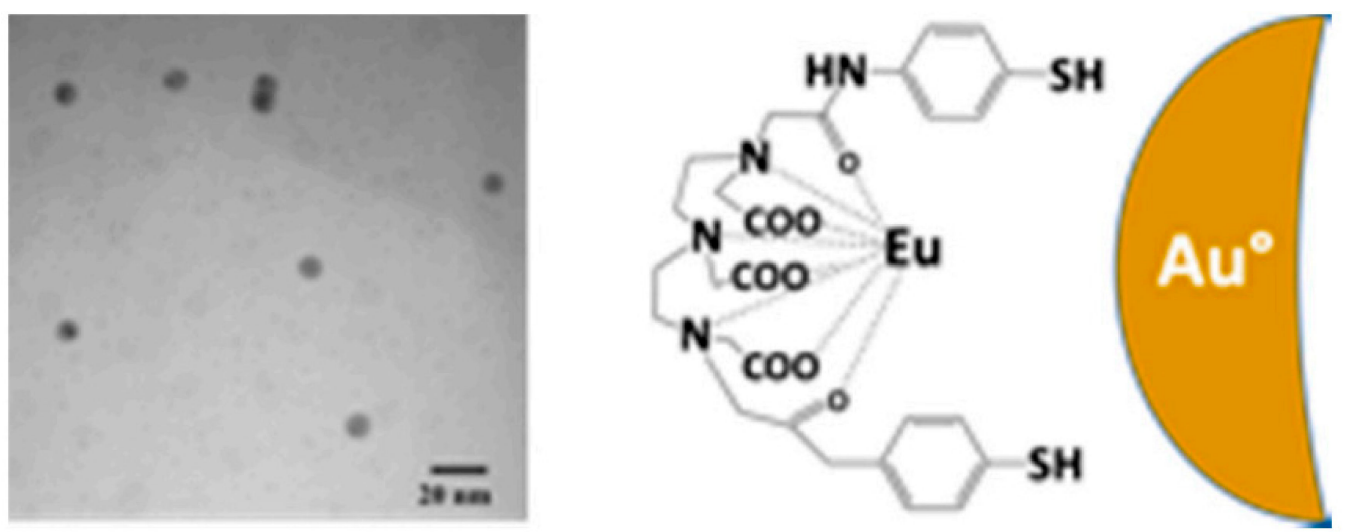

Figure 26. TEM micrographs of Au NPs of Eu (III) complexes as defined in ref. [166]. Reproduced by reference permission [186], RSC, 2006.

An alternative complex of europium of Au NPs rooted through a long alkyl chain has shown effective phosphate-anion sensing ability in water solution [187]. In this event, phosphate anions sensing was performed in successive steps by first attaching the Eu(III) complex of a non eluminescent nature; then, $\beta$-diketone was introduced to complex of high luminescence nature due to the interchange of the molecules of water with the $\beta$-diketone of the complex. Finally, the phosphate anion incorporation of luminescence took place. $\mathrm{Eu}(\mathrm{III})$ and $\mathrm{Tb}$ (III) complexes were also used for metal cation sensors [188]. Bipyridinecapped nanoparticles of mixed oxides were demonstrated to be extremely phosphorescent. Particularly, the incorporation of earth abundant metal ions and transition metal ions for $\mathrm{Eu}(\mathrm{III})$-based nanocomposites cause the reduction of the luminescence ability, because of the replacing of isomorphou of $\mathrm{Eu}$ (III) with these cations. The Gd(III) composites supported by particles were used in vivo, with magnetic resonance computed tomography and imagery (MRI) (see Figure 27) [189,190]. Robust improvement in the stems of MRI was observed due to the appropriate particle's nature for double imaging. In addition, $\mathrm{Gd}^{3+}$ complexes are being used for IRM applications [191-193].

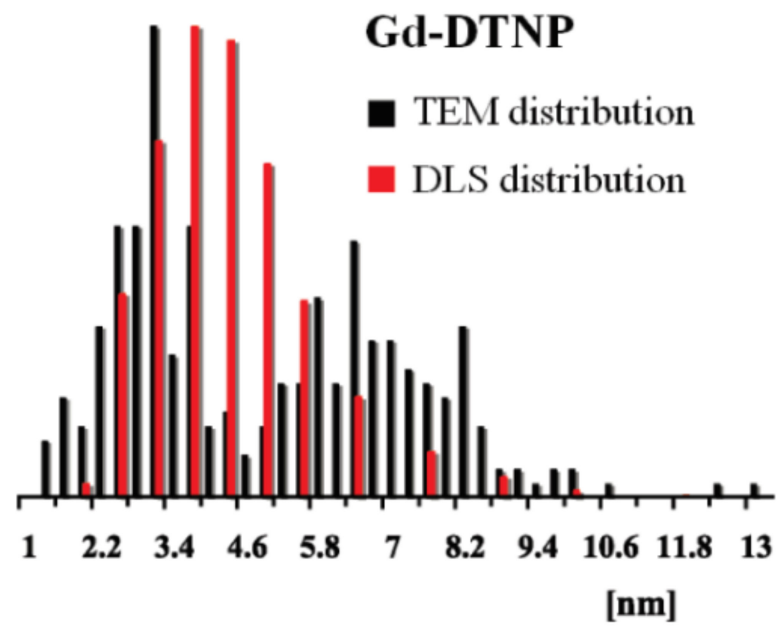

\section{Gd-Dt}

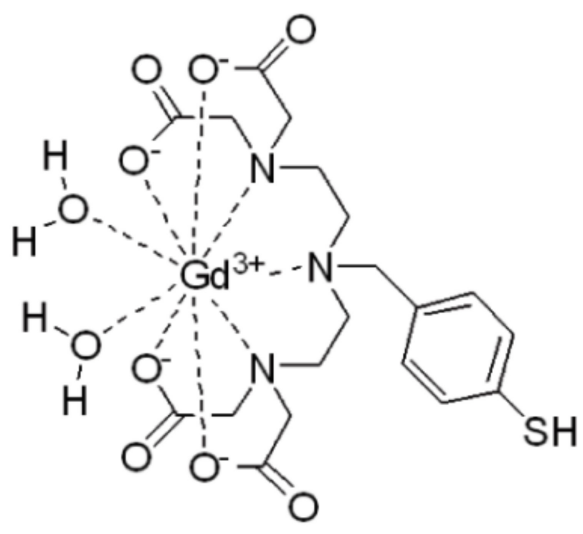

Figure 27. Au NPs of $\mathrm{Gd}^{3+}$ complexes for MRI uses as designated in [191]. Reproduced by reference permission [191], ACS, 2006. 


\section{Modification of Au NPs via Small Molecules for Biochemical Analysis}

Depending on their size, form and morphology, Au NPs demonstrate unique optical properties. The Au NPs solution shows different colours, depending on the scale [194]. $\mathrm{Au}$ NPs with targeted aggregation (typically $10-50 \mathrm{~nm}$ ) can cause changes in color in the solution from red to blue in the visible electromagnetic spectrum region. Due to the interpartum plasma relation, the color change in solution for Au NPs depends on the target stage, which is a unique phase in the preparations of visible sensors for a variety of biochemical analysis analysts. In comparison to other NPs, Au NPs have numerousbenefits, such as controlled synthesis [195], facile modification of the surface [196] and extraordinary molar absorption coefficient, which makes Au NPs the ideal nanoprobes for sensers application [197]. Moreover, because of their excellent biocompatibility and its low toxicity, Au NPs are capable candidate for in vivo imagery and antibacterial substances. The bioanalysis approach using the Au NP color change for the naked eye re-reading is the most common among all Au NP biosensors. The transition of Au NP states (dispersed to an aggregated one or vice versa) will disrupt the color of Au NPs, which makes for simple readings and no use of tools $[198,199]$. The change of its practical uses has been advanced by Au NPs-mediated biochemical analysis $[198,200]$. Many articles have been reviewed based on Au NPs mediated assays tonucleic acids, proteins and metal ions [201,202]. The surface modification of Au NPs with polymers or biomacromolecules such as proteins and nuclear acids has been discussed by some articles [203,204], while other reviews motivated the approaches for Au NPs functionalization of surface for biosensing uses by small molecules [205]. Because of the low steric hindrance and less complex assembly, bioconjugation of small molecules such as amino acids and sulfhydryl compounds are easily controlled on the surface of Au NPNPs, as compared to the bio-macromolecules such as as antibody, enzyme or polysaccharide. Furthermore, the small molecules' alignment and accessibility can be accurately organised without the loss of their biological activity; thefore, the functionalization of small-molecule supported Au NPs might favor repeatability and stability in the sensing of biochemical assays.

Since Au NP surface chemistry has great importance in biologic studies, the newest methods used for the Au NP surface changes with small molecules need to be checked and the difficulties and expectations relating to this process addressed. This analysis therefore focuses on the current production and functioning of surface chemical Au NPs in biochemical assays such as click chemistry, ligand exchange and the coordinating basis of small molecules (see Figure 28), and hence, the modification of the surface of Au NPs and their vital roles in Au NPNP mediated biosensors. In addition, the latest synthetic approaches for Au NPNP surface modicafication will also be discussed.

\subsection{Surface Conjugation of Au NPs by Click-Based Chemistry}

The preparation [206], bio-conjugation [207,208] and imaging [209] of polymers are some of the most exciting applications in materials science and biology. This is an outstanding approach for the modification of surfaces as it does not disturb the arrangement of NPs [210]. The 1.3-dipolar azide and alkyne (CuAAC) $\mathrm{Cu}(\mathrm{I})$-catalyzed reactions are studied primarily in click chemistry reactions [211]. This reaction takes place between azides and alkynes at the lowest temperature, or with a high temperature in the absence of $\mathrm{Cu}(\mathrm{I})$ [212]. It occurs at a high rate in water solutions in the presence of $\mathrm{Cu}(\mathrm{I})$, at room temperature. The constituents of $\mathrm{CuAAC}$ were used for the investigation of biochemical reactions and to bring about the modification of Au NPs to improve the chains of $\mathrm{CuAAC}$-facilitated assays that employ Au NPs for naked-eye display by employing thiol terminated azide and alkyne. The key focus is the improvement of CuACC-modified Au NPs for bio-chemical investigation; due to high selectivity of CuAAC and color alteration of Au NPs (from red to blue), the NPs of Au were improved by employing CuAAC for identifying $\mathrm{Cu}$ (II) [213]. The modification of Au NPs via Au-S bond using thiol-azide and thiol-alkyne and then reacted with CuAAC to alkyne and cross link azide resulted the accumulation of Au NPNPs, and this accumulation is accountable for the varying colour alteration from red to blue 
of $\mathrm{Au}$ NPs. Additionally, the aggregation depends on the sample $\mathrm{Cu}(\mathrm{I})$ material. In the reduction of $\mathrm{Cu}(\mathrm{II})$ into $\mathrm{Cu}$ (I) for the detection of $\mathrm{Cu}$ (II) (see Figure 29A), it is a quantitative conversion employing sodium ascorbate as a reducing agent and this reaction occurred within seconds. The good optical properties of click chemicals and Au NPs recognize the naked-eye recognition without any instrument of $\mathrm{Cu}(\mathrm{II})$, with a high selectivity and high sensitivity rate (sensitivity maximum LOD $=50 \mu \mathrm{M}$ ).

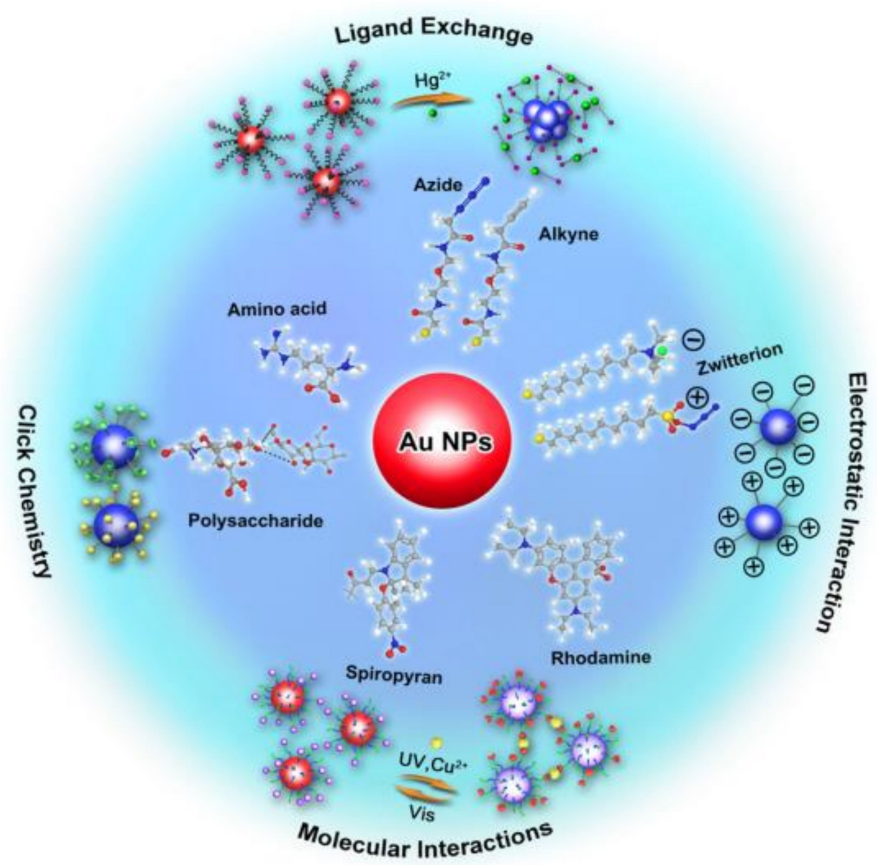

Figure 28. Surface chemistry strategy for the working and application in the biochemical study of $\mathrm{Au}$ NPs with small molecules. Reproduce with permission from reference [205], Copyright 2017, ACS.

A
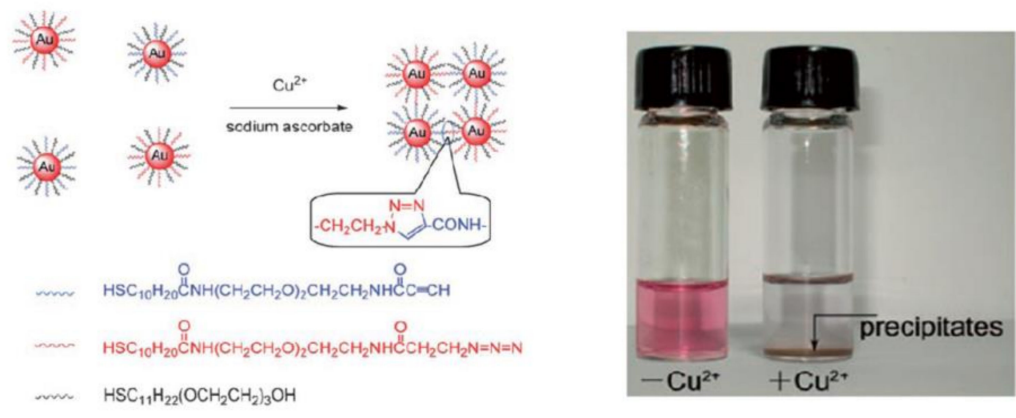

B
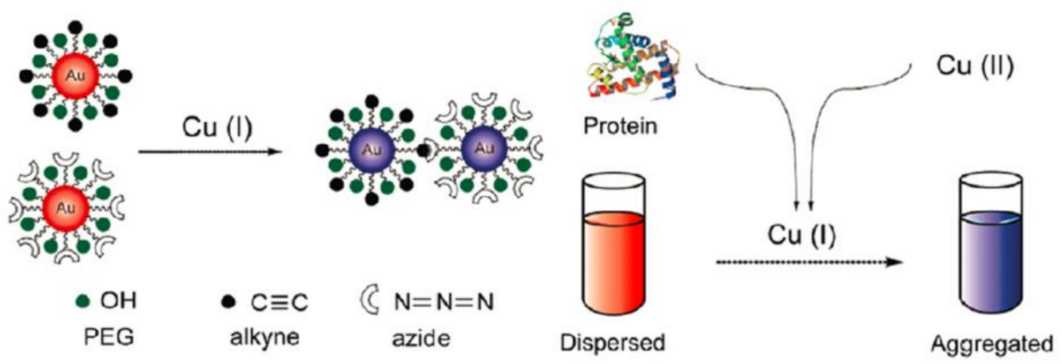

Figure 29. $\mathrm{Cu}(\mathrm{II})$ and protein visual identification of $\mathrm{CuAAC}$ arbitrated surface chemical $\mathrm{Au}$ NPs. In the presence of $\mathrm{Cu}(\mathrm{II})$, Au NPs are changed by (A) ligand- and alkyne-terminated, which can be taken into combination with CuAAC. Azideand alkyne-terminated ligands. Ataken with authorisation from ref [213], Copyright 2008, Wiley. (B) Surface alteration of CuAAC-mediated au-NPs for the quantification of proteins. 
Due to this procedure, a lot of research has been conducted for highly sensitive and selective $\mathrm{Cu}(\mathrm{II})$ naked-eye detection and reduction of $\mathrm{Au}$ NPs and $\mathrm{CuAAC}$. CuAACmediated NPs of Au assays can be used for the identification of reductants. The peptide bond, as a reductant in nature, led $\mathrm{Cu}(\mathrm{II})$ to $\mathrm{Cu}(\mathrm{I})$ in proteins. In order to detect complete samples of proteins $(\mathrm{g} / \mathrm{mL}$ ), this CuAAC-arbitrated au-NPs test can be used [214] (see Figure 29B). Many detergents and illegitimate chemicals, such as melamines, are used to encourage the total amount of protein, such as whole milk, in difficult samples. The $\mathrm{CuAAC}$-mediated immuno-assays, employing functional Au NPs for the identification of various kinds of disease biomarkers, as widely used. Since huge collections are currently in existence, an enzyme-supporting immuno sorbent test (ELISA) has been commonly used to recognize disease biomarkers, food quality control and atmospheric observation as a biomarker. While typical ELISA is somewhat effective, its disadvantages are also apparent, including instrument-dependency, high price and long time duration. In order to avoid these problems and facilitate good immune testing, mainly for naked-eye recognition, a typical enzyme-labeled antibody is substituted by copper oxide nanoparticles $(\mathrm{CuO}$ NP) [215]. The volume of $\mathrm{Cu}(\mathrm{II})$ issued from labeled antibodies-CuO-NP depicted the target level in the samples by immune response. Therefore, the accumulation level of $\mathrm{Au}$ NPs affected by $\mathrm{CuACC}$ indicates the target quantity, which can be reputed by antigenantibody communication (see Figure 30A). The mentioned process has been used in actual serum samples for the $100 \%$ detection of human immuno-deficiency virus antibody. The named NP-CuO antibody instead of the enzyme is cheaper and more powerful for mass processing than the biological enzyme. The fundamental drawback, however, is that the antibody combination in $\mathrm{CuO}-\mathrm{NPs}$ changes the antibody efficiency. The exact binding point of the antibody is not definite; it cannot be ignored the possibility of $\mathrm{CuO}$ being bound to the antibody binding surface (Fab), which impedes the antibody efficiency marking. We have advanced an immunoassay that is market-accessible with built into labeled alkaline phosphatase (ALP) and CuAac-supported scheme for the deeper and naked-eye recognition, to account for the problem of reducing antibody activity by changing $\mathrm{CuO}-$ NP [216] (see Figure 30B). ALP is the most common labeling enzyme in immunoassays for substitution of the CuO NP-labeled secondary antibody with ALP-labeling secondary antibody. ALP-bound secondary antikoids are commonly supplied by labeling antibody dealers so that this conjugation does not distract the antibody Fab area that the main siteof-the antibody is changed. Furthermore, dephosphorylation catalyzed by ALP reduces $\mathrm{Cu}(\mathrm{II})$ to $\mathrm{Cu}(\mathrm{I})$ to ascorbic acid by not reducing ascorbic acid phosphate. The reaction of CuAAC leads to accumulation Au NPs and change in the color of Au NPs shows the quantity of goal in the sample. This CuAAC-based Au NPs fulfilled immuno-assay has a high sensitivity ratio for naked-eye detction relative to typical ELISA due to the "click" and "enzyme" amplification. It can be used to find the infection in the patient's serum by myco plasma pneumoniae (MP) as accurate as $100 \%$, while $50 \%$ forpredictable ELISA. The remaining immunoassays are more adaptable to the immuno support based on an anti-body modified $\mathrm{CuO}-\mathrm{NP}$, since nanomaterials do not require straight adjustment to either the antigen or the antibody. The wide accessibility of antibodies with ALP-labeling permits and examined for beneficial naked-eye readout of all immuno-assays. 

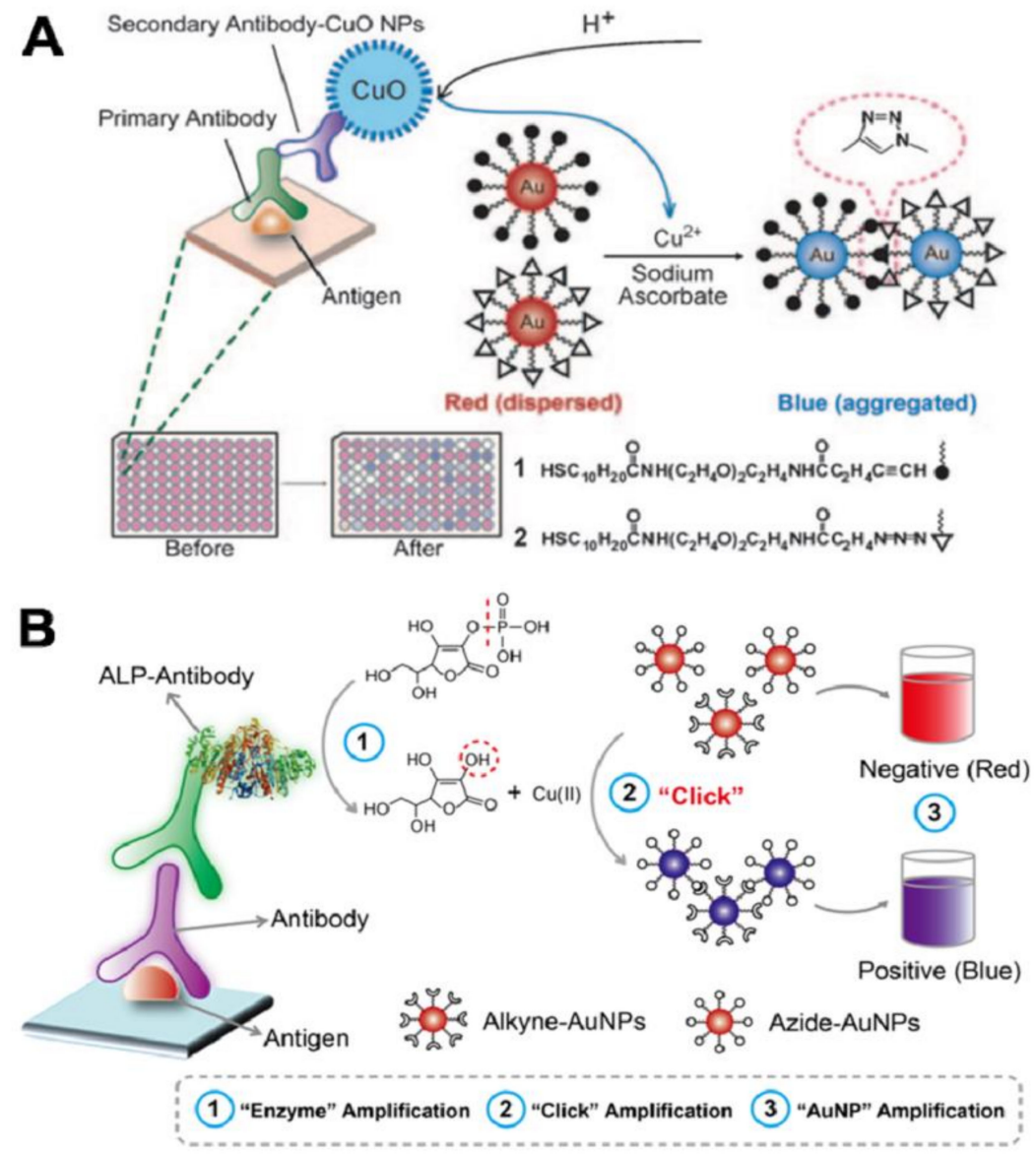

Figure 30. Surface changes in $\mathrm{Cu}-\mathrm{AAC}$ immunosurveys for Au NP. (A) Anti-CuO and azide and alkyne-modifited au-NPs based immuno-assays. (A) CuO NPs have been dissolved to release copper ions, which have caused Au-via NP's CuAAC to be aggregated. (B) Immuno assay between azide and alkyne-functionalized Au NPs based on ALP-triggered CuAAC. Copyright 2011, Wiley, adapted with reference permission [215].

\subsection{Modification of Au NPs via Co-Ordination Based Recognition}

\subsubsection{Modification via Amino Acids}

The surface of Au NPs can be modified with large number of amino acids via the $\mathrm{Au}-\mathrm{S}$ bond, by having resilient connections with ions of metal, such as $\mathrm{Hg}^{2+}$ or $\mathrm{Pb}^{2+}$. $\mathrm{Hg}^{2+}$ is coordinated at the C-terminal of lysine and arginine with the aid of $-\mathrm{NH}_{2}$ and $-\mathrm{COOH}$ and Arg-Au NPs (Arg-Au NPs) can be modified by the Au NPs with arginine, so that Arg-Au-nPs are accumulated at a speedy rate. Numerous variables can be accessed to yield the diverse outputs of logic gate scheme. The intended useful colorimetric gates rely on the unique binding effects between amino acids such as arginine, lysine and cysteine and metal sucha ions $\left(\mathrm{Hg}^{2+}\right.$ and $\left.\mathrm{Cr}^{3+}\right)$ [217] (see Figure 31A). Molecular gates logic [217] can practise biochemical "inputs" to produce "outputs" built on connections with molecules like recognition based on coordination, that is theoretically beneficial for biochemical analyses. Arginine and $\mathrm{Hg}^{2+}$ are the two inputs sets and color of solution of Au NPs is the output set. The " 0 " input signifies the lack and " 1 " denotes the existence of arginine or $\mathrm{Hg}^{2+}$. The output " 0 " denotes red (dispersion), whereas " 1 " signifies blue (accumulation) of the Au NPs. A calorimetric and gate can be produced in the presence of both arginine and $\mathrm{Hg}^{2+}$, the Au NPs will aggregate and display a blue color (the output is "1"). However, predictable molecular gates of logic implemented in bulk frequently need knowledge and ingest chemicals sufficiently. To overcome this difficulty, microfluidic technology was presented [218] to exhibit the gates of molecular logic and it has demonstrated advantages of automatic operation and notable information on the logic gates (see Figure 31B). 
A

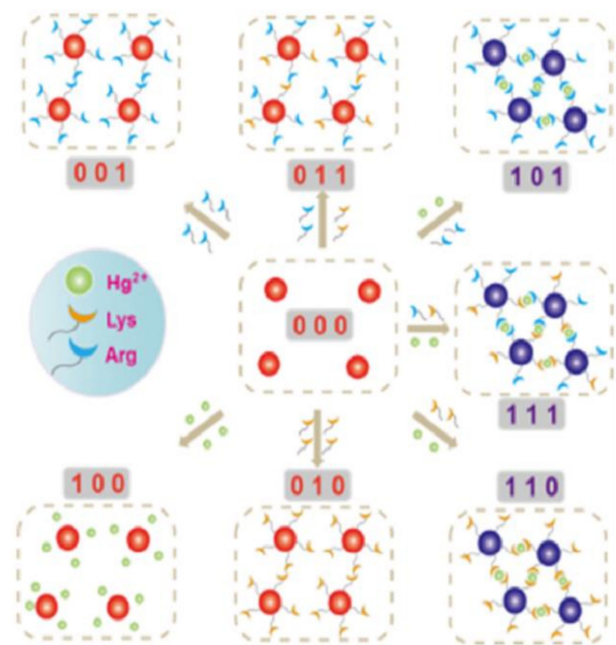

B

(1)
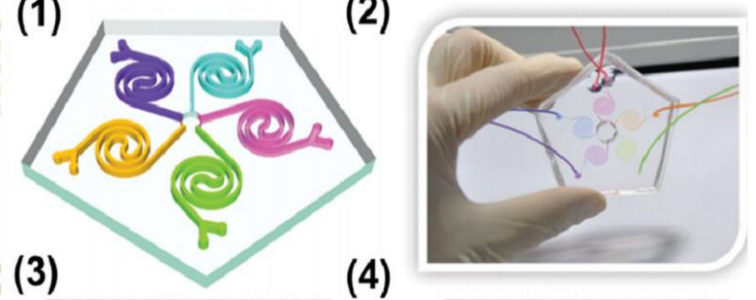

(4)


Figure 31. Modification of Au NPs for biochemical analysis by amino acid mediated surface. (A) A colorimetric (AND+OR) logic scheme that uses Au NPs with arginine/lysine. (B) Microfluidic systems for metal ion detection combined with molecular logic gates. Schematic diagram and microfluidic chip photographs are presented in panels (1) and (2). TEM photos and the required observation window color of the Au NPs are in panels (3) and (4). Copyright 2014, Wiley, adapted by reference permission [217], Copyright 2014, Wiley.

Recognition based on the coordination, the arginine-modified Au NPs can be also applied for glutathione (GSH) finding. A dispersion controlled chromogenic approach which is another method), was established for the emphasis on the target-led dispersion of GSH-based Au NPs based on $\mathrm{Hg}^{2+}$ facilitated the Arg-Au-accumulation of NPs. Due to the metal ions molecular interactions with amino acids, the $\mathrm{Hg}^{2+}$ canresult the accumulation of Arg-Au NPs. Addition of GSH avoid the accumulation of Arg-Au NPs due to particular interaction of GSH with $\mathrm{Hg}^{2+}$; therefore, for GSH finding in biological samples, a biosensor can be built [219] (see Figure 32).

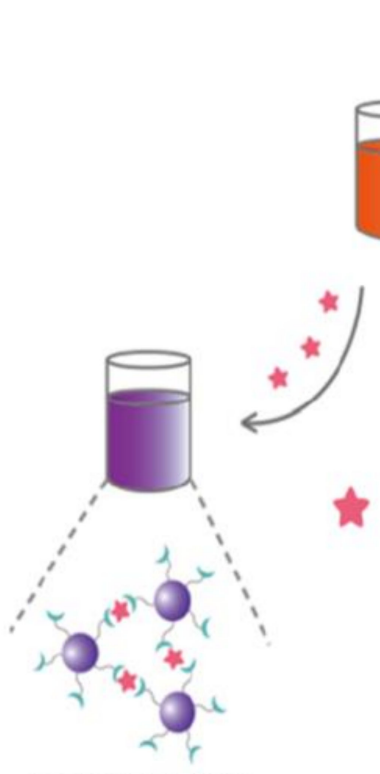

Arg-AuNPs $+\mathrm{Hg}$ (II)
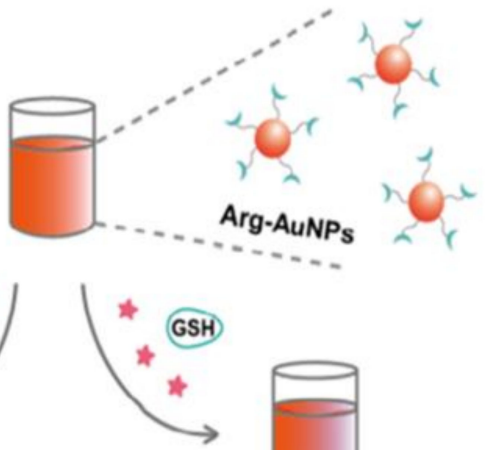

$\mathrm{Hg}(\mathrm{II})$

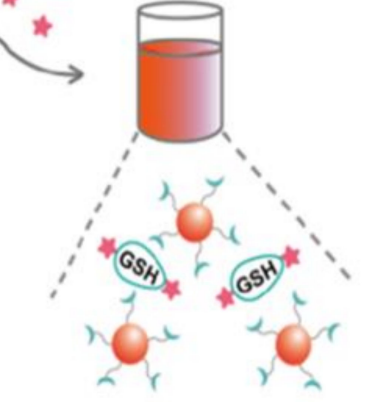

Arg-AuNPs + Hg(II) + GSH

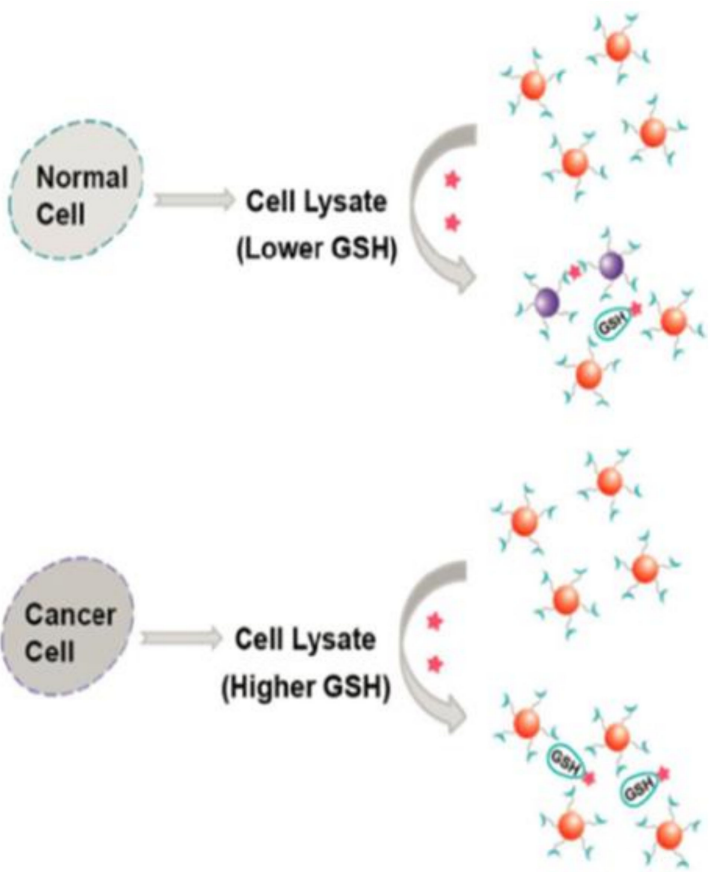

Figure 32. Arginine-modified Au NPs for GSH sensing in cell cancer are distributed regulated by chromogenic approach. Adapted by reference permission [219], Copyright 2015, Wiley. 
Due to the detecting LOD of GSH $10.9 \times 10^{-9} \mathrm{M}$, this process can be utilized for estimatation and measurement of GSH levels in cell lysates are more notable than normal cells in cellular cancer cells. In spite of the fact that, restricted thinks about account, the in vivo finding utilize Au NPs for naked-eye readout, taking into consideration that the cell level infinitesimal pictures grounded on Au NPs might not be existing since of their nanometric molecule estimate and it needs plenty of Au NPs to driven within the obvious color alter. Another method colorimetric measure based on cysteine interceded accumulation of Au NPs has been presented [215] (see Figure 33A). The amino acid cysteine encompasses a strange structure, having thiol Moity (-SH) at one side and $\mathrm{COO}-$ and $\mathrm{NH}^{2+}$ functional groups on other side. By means of the $\mathrm{Au}-\mathrm{S}$ bond, cysteine can be attached to the Au NPs and the Au NPs can be bridged by the electrostatic contact between the positive and negative charged groups that aggregates the Au NPs. The cysteine oxidation by iodide to produce disulfide cysteine prevents Au NP aggregation. An HRP-mediated iodide catalyzed cascade method was introduced to control the dispersion and aggregation of Au NPs, which endorsed the naked-eye identification of details, in conjunction with this catalytic method with HRP catalyzed reactions. This immuno assay was utilized to examine the hepatitis-C virus antibodies in real blood sample via a naked-eye finding precision of $100 \%$, while predictable ELISA is $20 \%$, suggesting that this Au NP-based immuno assay enables high sensitivity and naked-eye knowledge for biomedical diagnostics. The $\mathrm{Cd}^{2+}$ and GSH-Au NPs organization authorizes the recognition of cadmium ions $\left(\mathrm{Cd}^{2+}\right)$ [220] (see Figure 33B). In concentrated $\mathrm{NaCl}$ solution, the unmodified Au NPs easily aggregate, but the presence of GSH prevents the aggregation of salt induced Au NPs. Once the Cd ${ }^{2+}$ is applied to a firm blend of $\mathrm{Au} \mathrm{NPs}, \mathrm{GSH}$ and the $\mathrm{NaCl}$, one of the $\mathrm{Cd}^{2+}$ will bind with $4 \times \mathrm{GSH}$, reducing the amount of free GSH on the Au NPs in order to weaken the integrity and aggregation of the Au NPs. In water and assimilated rice samples, this colorimetric method can effectively perceive $\mathrm{Cd}^{2+}$ with high precision and suitable procedures.

\subsubsection{Modification via Sulfhydryl Compounds}

The chelation contact among spiropyran (SP) and the $\mathrm{Cu}^{2+}$ could be changed via UV-light and the SP could be isomerized to planar and open shape of merocyanine after UV-light irradiations (MC). Rather than retaining in the nonplanar and sealed SP type, it is possible to prepare and use spiropyran-modified Au NPs (spiropyran-Au NPs) to render a re-settable and multi-reading logic device proficient in numerous kinds of logic procedures built on the accumulation of Au NPs in water as solvent [221] (see Figure 34A). To appear red in color, Spiropyran-Au NPs were monodispersed in solutions. Spiropyran transforms into open merocyanine through exposure to UV, in such a manner that the distributed merocyanine-modified Au NPs (MC-Au NPs) are aggregated by change in color from red to purple due to merocyanine and $\mathrm{Cu}^{2+}$ chelation. Significantly, by visible light, the MC-Au NPs could simply return to the SP, indicating that the Au NPs-based logic system can be reset for different periods of discovery (see Figure 34B). In environment monitoring, identification of $\mathrm{Cr}^{3+}$ and $\mathrm{Cr}_{2} \mathrm{O}_{7}{ }^{2-}$ is very significant. For the identification of both $\mathrm{Cr}^{3+}$ and $\mathrm{Cr}_{2} \mathrm{O}_{7}{ }^{2-}$ [222] meso-2,3-dimercaptosuccinic acid (DMSA) was used to adjust Au NPs (see Figure 35A). In order to prepare the Au NPs, DMSA with two -SH functional groups and two - $\mathrm{COOH}$ groups was used for stabilization and reduction. To detect $\mathrm{Cr}^{3+}$ in the DMSA, the -COOH groups can be used. In the meantime, one $\mathrm{Cr}_{2} \mathrm{O}_{7}{ }^{2-}$ intermingles with the two DMSA-Au NPs via the ending carboxyl-groups of DMSA molecules through close $\mathrm{OH} \cdots . . \mathrm{O}$ hydrogen bonds. The density-functional-theory may measure the change in Gibbs free-energy $(\Delta G)$ of the contacts between DMSA-Au NPs and the various metal ions. Among all of the metal ions, the lowest $\mathrm{G}$ for $\mathrm{Cr}^{3+}$ and $\mathrm{Cr}_{2} \mathrm{O}_{7}{ }^{2-}$ shows that DMSA-Au NPs have optimum affinity for both of the hydrated $\mathrm{Cr}^{3+}$ and $\mathrm{Cr}_{2} \mathrm{O}_{7}{ }^{2-}$. The naked-eye LOD is $10 \mathrm{nM}$, slightly smaller than the requirements of the Environmental Protection Agency (EPA) (EPA-3060A). This approach has been effectively utilized for finding $\mathrm{Cr}^{3+}$ from the chromium slag dumpsite in chromium-polluted soil. 


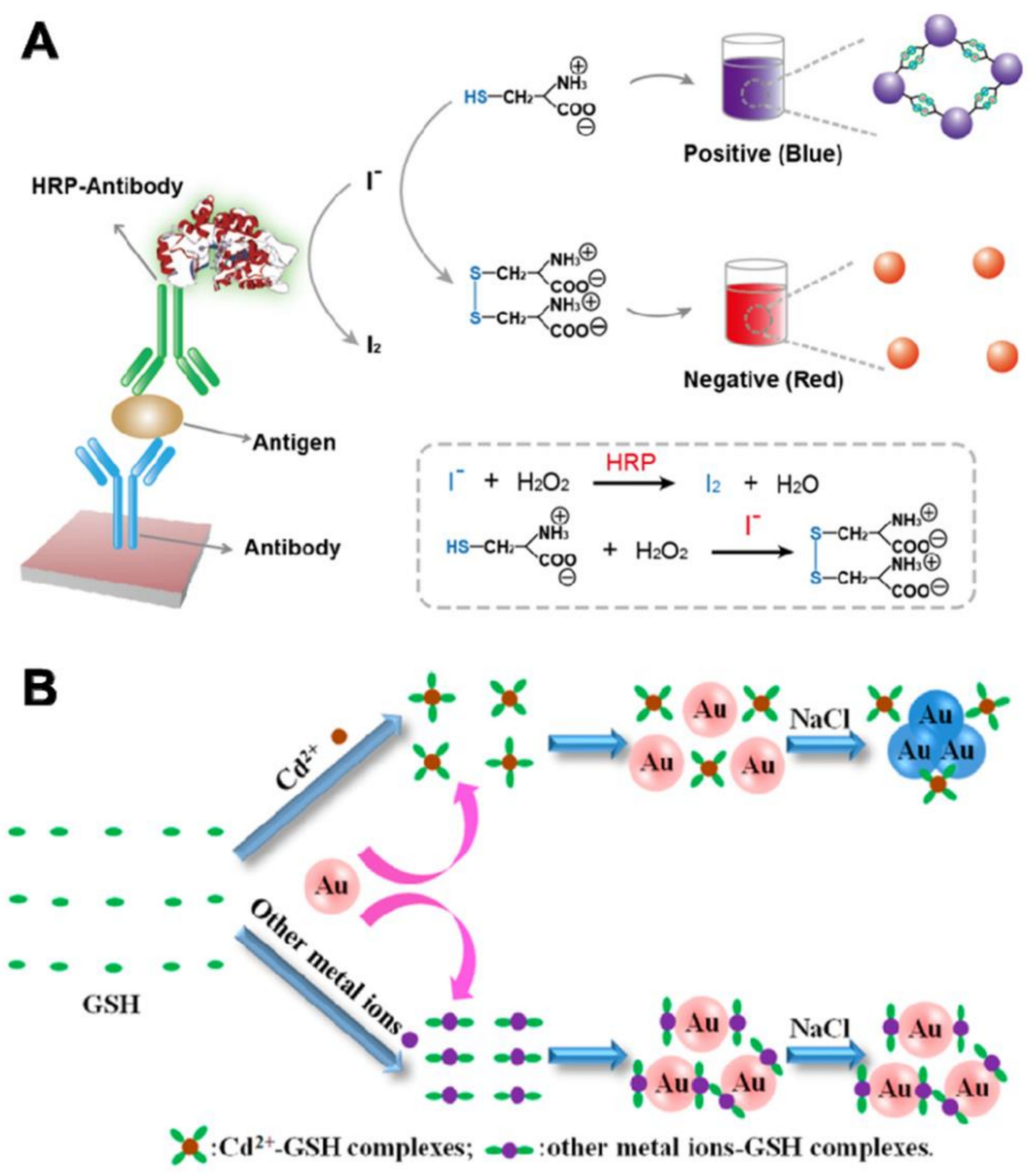

Figure 33. For naked-eye study, amino acids mediated surface-modification of the Au NPs. (A) HRP mediated immunoassay modulation of Au NPs. Iodide catalyzed cysteine oxidation will alter Au NPs' surface chemistry and influence Au NPs' aggregation and dispersion. (B) The complex of $\mathrm{Cd}^{2+}$ and GSH can alter surface morphology of Au NPs and increase stability of the citrate-Au NPs at change in concentration of $\mathrm{NaCl}$. Adapted with permission from reference [220]. Copyright 2014, ACS

Rapid and straight forward detection of the tri-valent metal ions $\left(\mathrm{M}^{3+}\right)$ is vital and interesting, additionally to detecting individual ions. The researchers designed Au NPsmodified zwitterionic molecule ( $\mathrm{Zw}-\mathrm{Au} \mathrm{NPs}$ ). The aggregation of $\mathrm{Zw}-\mathrm{Au}$ NPs with color shift could be caused by the $\mathrm{M}^{3+}$, like $\mathrm{Al}^{3+}, \mathrm{Fe}^{3+}$ and $\mathrm{Cr}^{3+}$ [223] (see Figure 35B). In addition, due to various forms of the trivalent ions at diverse $\mathrm{pH}$ values, the $\mathrm{Zw}-\mathrm{Au}$ NPs can be retrieved. The $\mathrm{M}^{3+}$ could coordinate with the $\mathrm{Zw}-\mathrm{Au}$ NPs and cause their aggregation in acidic or neutral environments. The free $\mathrm{M}^{3+}$ turns into $\mathrm{M}(\mathrm{OH})_{3}$ in the simple solution and avoids the contact among $\mathrm{Zw}-\mathrm{Au} \mathrm{NPs}$ and the $\mathrm{M}^{3+}$, following the re-dispersion of $\mathrm{Zw}-\mathrm{Au}$ NPs. The instrumental-free and low-cost features made this examination suitable for use in resource-limited components, compared to earlier accounts on $\mathrm{M}^{3+}$ findings. 
A

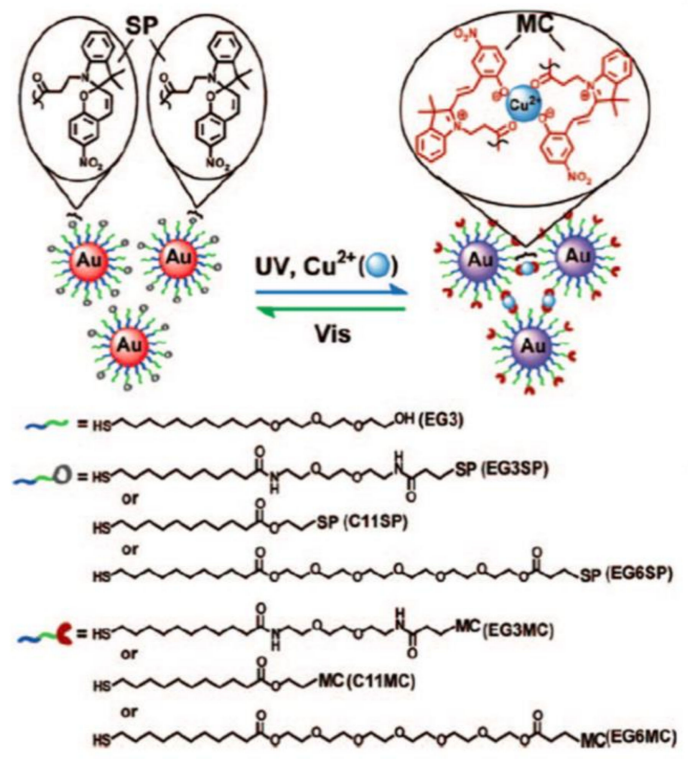

B

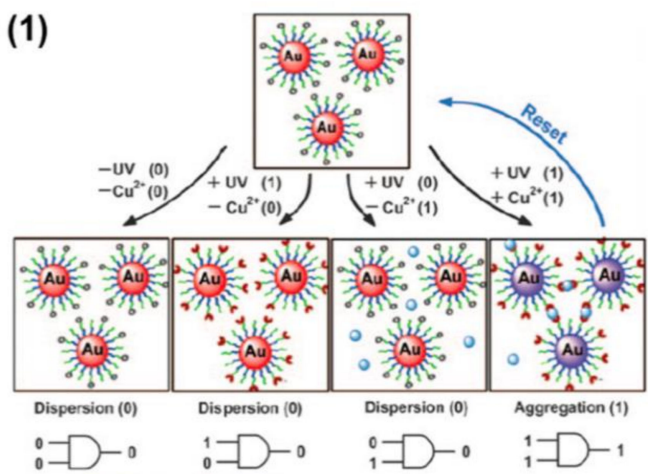

(2)

(3)

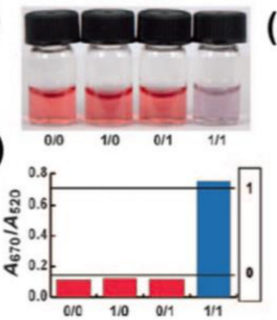

(4)



Figure 34. Based on photoresponsive power, spiropyran-functionalized Au NPs realize resettable, multi-readout logic gates. (A) The suggested photoresponsive control mechanism for spiropyran-Au NPs. (B) Resettable spiropyran-Au NPs-based logic gates. (1) Color of spiropyran-Au NPs solutions (1/1) switched from red to purple. (2) Pictures of Au NP solutions and (3) their corresponding absorption of UV/vis. (4) The AND Logic Gate's Truth Table. Adapted from reference with permission [221], Copyright 2011, Wiley.

A

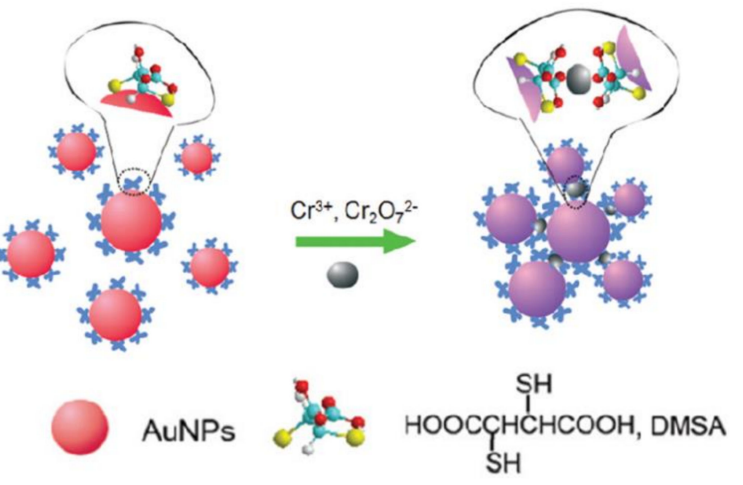

B $\ominus$

$\bigoplus$
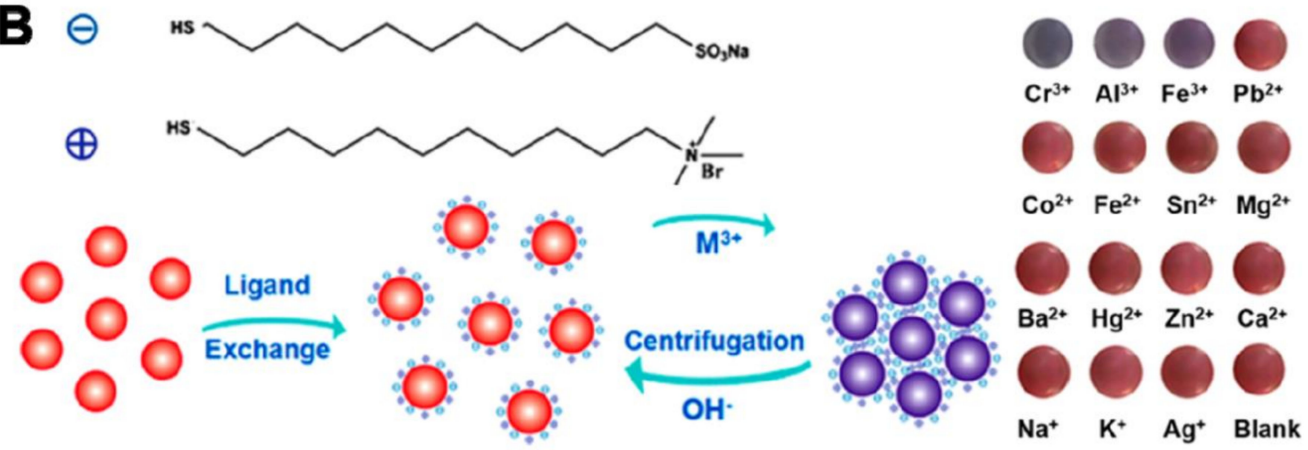

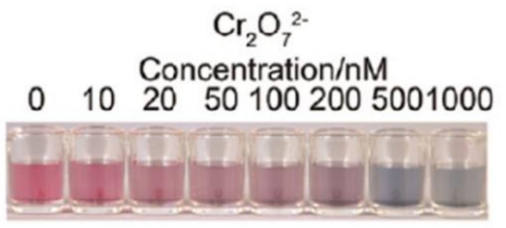

$\mathrm{Cr}^{3+}, \mathrm{Cr}_{2} \mathrm{O}_{7}^{2 \cdot}$ mixture

Concentration/nM

o 1020501002005001000

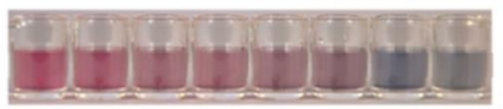

Figure 35. Sulfhydryl compound-modified Au NPs visual biosensors for heavy metal ion detection. (A) DMSAfunctionalized Au NPs for both the $\mathrm{Cr}(\mathrm{III})$ and $\mathrm{Cr}(\mathrm{VI})$ fast detection. (B) To adjust the Au NPs to produce inter-molecular zwitterionic $(\mathrm{Zw})$ surfaces, mixed charge thiols are employed. The $\mathrm{M}^{3+}$ can efficiently cause aggregation of the $\mathrm{Zw}-\mathrm{Au}$ NPs by interfere with their surface-potential, like $\mathrm{Fe}^{3+}, \mathrm{Al}^{3+}$ and $\mathrm{Cr}^{3+}$ and the aggregated Au NPs could be re-generated and re-cycled via removing $\mathrm{M}^{3++}$. Adapted with permission from reference [223], Copyright 2016, ACS. 


\subsection{Modification via Ligand Exchange for Biochemical Analysis}

The Ligand's exchange for modification of Au NPs surfaces is the best option for the construction of biosensors. $\mathrm{Hg}^{2+}$ and essential biochemical markers were identified using the various ligand exchange reactions. To change the Au NPs, first presumed a quaternary $\mathrm{NH}^{4+}$ group ended thiol (QA-SH) and synthesized the QA-Au NPs via the Au-S bond [224] (see Figure 36A). The QA-Au NPs exhibit high stability in acidic solution because of the repulsive forces among the charged quaternary $\mathrm{NH}^{4+}$ groups of Au NPs. The greater interaction of mercury (II) $\left(\mathrm{Hg}^{2+}\right)$ with the -SH group when compared to QA-Au NPs could replace the Au NPs with the QA group, leading to the Au NPs aggregation because of the thiol terminated QA deprotection. The aggregation degree is related to the $\mathrm{Hg}^{2+}$ concentration. The QA-Au NPs can therefore allow naked-eye detection of $\mathrm{Hg}^{2+}$ in aqueous systems with a low LOD of $30 \mathrm{nM}$, substantially less than the standard value for drinking water, as fixed by the World Health Organization (WHO). In addition, by adjusting rhodamine B dye isothiocyanate (RBITC) and the poly(ethylene glycol) (PEG) on $\mathrm{Au}$ NPs for the recognition of $\mathrm{Hg}^{2+}$ with exceptional strength, specificity and sensitivity, we planned a recyclable review [225] (see Figure 36B). Only $\mathrm{Hg}^{2+}$ can dislocate RBITC from the $\mathrm{Au}$ NPs, referring to RBITC, which is initially quenched via Au NPs, to boost fluorescence. To help Au NPs stay stable and monodisperse in actual samples, the thiol terminated PEG connects to the remaining active sites of Au NPs. The LOD of this attempt for $\mathrm{Hg}^{2+}$ $(2.3 \mathrm{nM})$ was less than both the extreme limits shown by EPA and legalized by the WHO. The efficacy of this investigation was confirmed when $\mathrm{Hg}^{2+}$ was tested in the complicated samples, such as river water and the living cells. This method may also discover thiols and other targets. In order to find acetyl-cholinesterase (AChE), a bio-marker for the Alzheimer's disease, the rhodamine B modified Au NPs (RB-Au NPs) was synthesized (AD). The Au NPs will physically adsorb Rhodamine B and the Au NPs have quenched the fluorescence of RB. The addition of AChE brings its substrate (acetyl-thiocholine (ATC)) hydrolysis into thio-choline, that displaces the RB from Au NPs via stronger Au-S bonds in comparison to the physical adsorption between RB and Au NPs [226] (see Figure 36C). The RB fluorescence that can be utilized for quantitative recognition of AChE, increases after these ligand-exchange reactions. The accumulation of the distributed Au NPs results from the interaction between the thiocholine that is positively chargeable and the au-NP that is negatively charged. This vary in status of Au NPs (from scattered to aggregate) contributes to the color transform of Au NPs (red to blue) that could also be utilized to find $\mathrm{AChE}$ as visual indicator. A responsive and select test for at least $0.1 \mathrm{mU} / \mathrm{mL}$ of AChE in brain/spinal fluid of the transgenic AD mouse distress was provided by colorimetric and fluorometric details. This RBAu NPs-based assay might be hopeful for checking AChE in the human CSF for initial identification and prediction of AD. Organophosphorus and carbamate pesticides can be detected by RB-Au NPs in the food samples because both of these common organophosphate pesticides can efficiently prevent AChE movement [227] (see Figure 36D). If these organophosphorus pesticides contaminate the food samples, ATC hydrolysis into thiocholine will not occur with $\mathrm{AChE}$, thiocholine will result in electrostatic interactions to aggregate au-NPs. This test knowledge is thus the opposite of the AChE test, since the negative sample is shown by the higher fluorescence and blue colour. The LOD was sufficient both with the naked eye and fluorescence signals to reach the highest residual limit (MRL), as obligatory by the EPO. Relative to the conventional procedures, the key advantage of this system is that the double information system that could perceive various needs, where fluorescence signals could be employed as a quantitative indicator in huge testing laboratories and the color of the Au NPs could be employed as the qualitative indication to recognize on-site testing. 
A

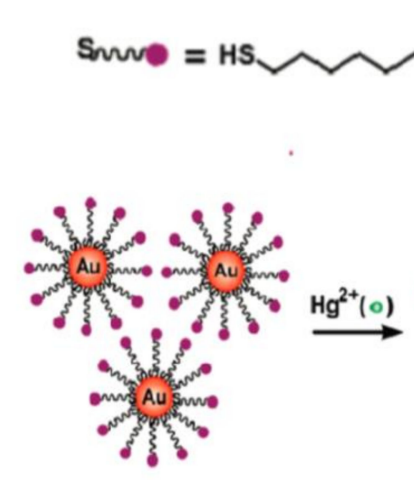

C

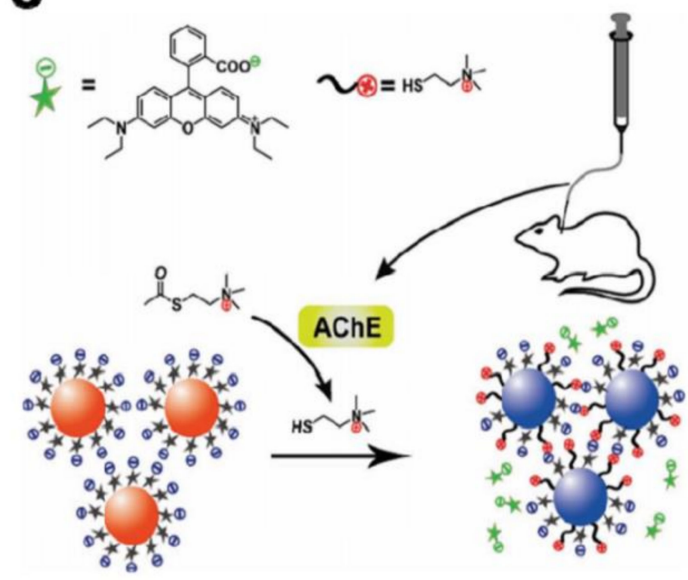

B

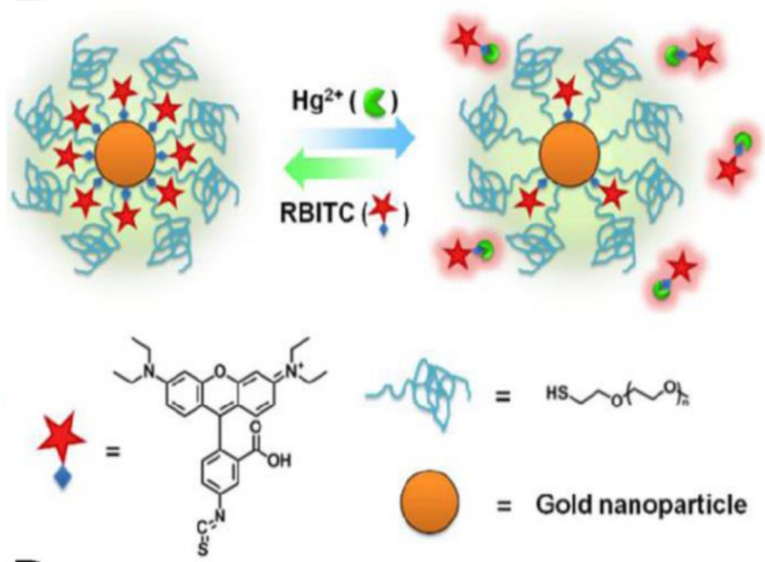

D
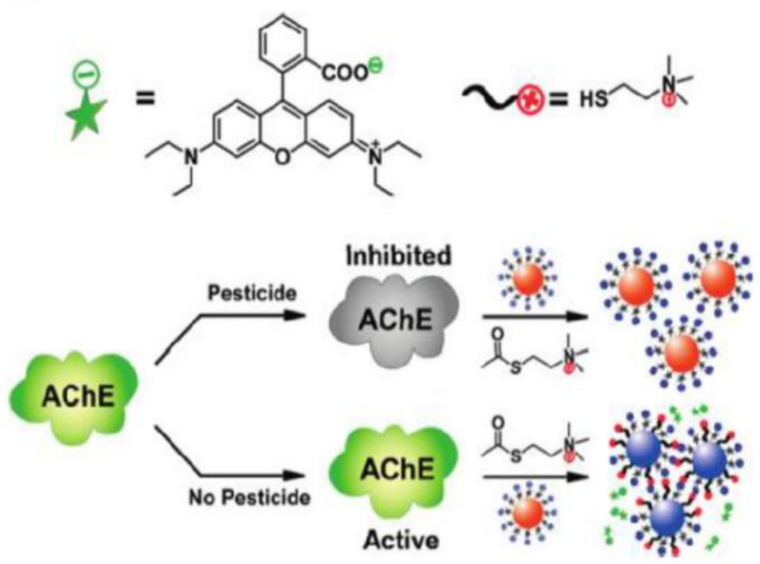

Figure 36. Ligand's biodetection surface change exchange approach for Au NPs. (A) Au NPs for the $\mathrm{Hg}^{2+}$ detection of ammonium quaternary group-capped. (B) Poly (ethylene glycol) (PEG)-comodified Au NPs for detection of $\mathrm{Hg}^{2+}$ Rhodamine B isothiocyanate (RBITC). (C) Modification of the rhodamine B Au NP test in cerebrospinal fluid of transgenic mouse with Alzheimer's disease in dual read-off acetylcholinesterase. (D) Dual readings for organophosphorus and carbamate pesticide detection in Rhodamin B-Au NPs. Adapted by reference permission [226]. Copyright 2012, Wiley.

\section{Interaction of Biomolecules with Au NPs for Catalysis}

\subsection{Interaction of DNA}

The researchers were able to use simple rules to design and control DNA montage structures by discovering the DNA double-helical structure and existence of the base coupling that hold it jointly [228]. DNA synthesis technology has also made it possible to generate DNA strands of up to 100 base lengths cheaply and easily. Various variations in the DNA backbone and terminal can be used to bind moyeties of specifically other molecules of concern such as phosphorothioates, amines, thiol groups and biotine. All these developments allowed the design of new DNA structures that can be placed with precision sub-nanometer, modified from those found in the wild and enhanced with particles, such as Au NP [229-232]. There are many ways to bind Au NPs to DNA templates with a double stranding (ds). A modified DNA phosphorothioate was used to bind Au NPs in a (ds)-DNA backbone to particular points using the bifunctional molecule "molecular fixer," induced by NN'bis(alle-iodoacetyl)-2-2'-dithiobis(ethylamine) (BIDBE) (see Figure 37) $[233,234]$. 

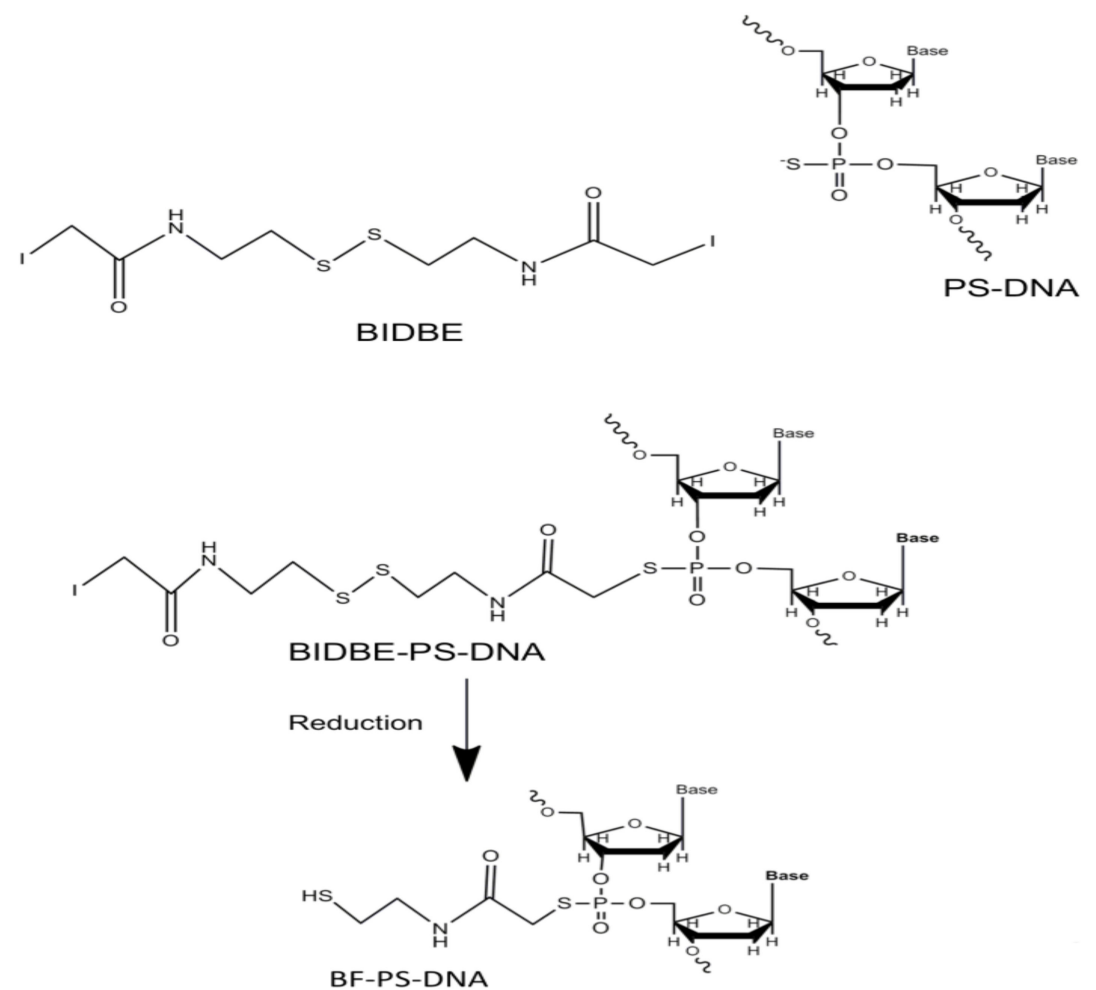

Figure 37. BIDBE binds to BIDBE-PS-DNA (phosphorothioate) to form the bi-functional linker (BF), associated with PS-DNA (bifunctional linker). BF supplies an au-NP sulfhydryl group. Reprinted with reference authorisation [233], Copyright 2007, WILEY-VCH.

Maybe the most basic template (ds)- DNA can be used to generate support for the development of electronic and photonic rods [235], sensor devices [236] or as a catalyst [237]. Definitely, while canonic Au NPs are classically roughly spherical, Au nanorods' catalytic strength has previously been taken [238] and several reports of the making of au nanowires with DNA template [239-244]. Due to its great uniformity and density over broad areas of $\mathrm{Au} \mathrm{NP}$, the catalytic significance of the arrays, synthesized by procedures not using bio-templates, was shown [245]. DNA can provide such a prototype as it could be planned to produce self-assembled styles that could regularly shape a wide surface. In addition, tiling by double cross over DNA styles is used for previous examples of the synthesis of the intended DNA nanostructures (see Figure 38) [246].

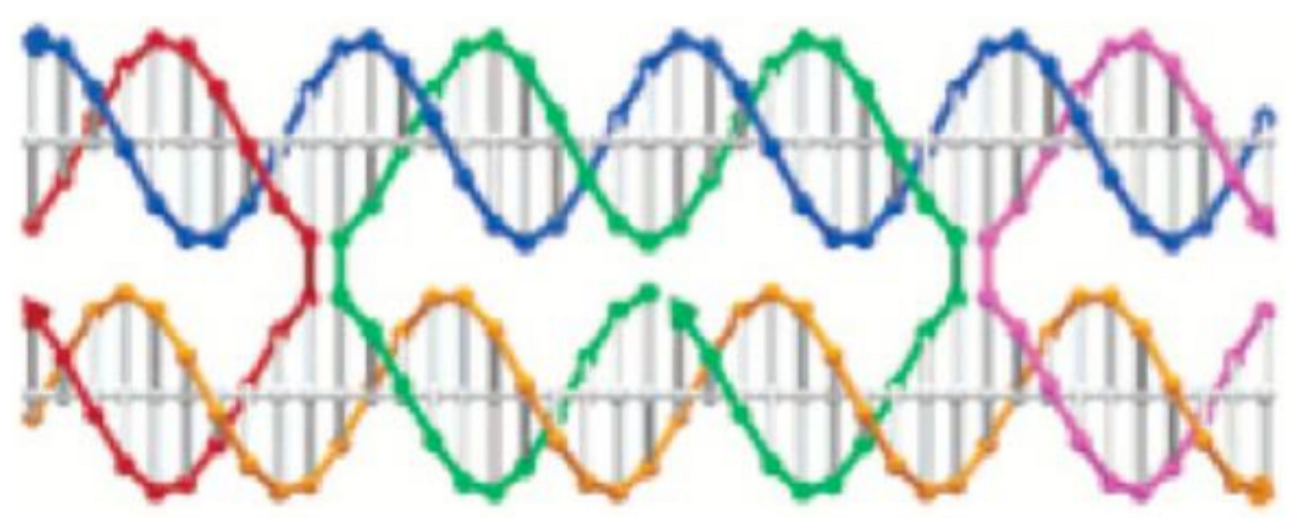

Figure 38. A DX DNA schematic. The color is different for each individual strand of DNA. The location of any phosphate is represented by small circles and the upright grey lines are the base-pairs. The red, green and pink strands overlap between the two double helices. Reprinted with reference authorisation [247], Copyright 2004, ACS. 
The DX-motives consist of two double DNA strands linked to the nearby double beach by crossing single beams from one double beam. DX DNA provides the essential hardness of DNA tiles if a wide surface area of reduced distortion is periodically dissolved. DX DNA was considered for a thin, rigid andtri-angular configuration with "sticky ends" and can frequently can cover large 2D surfaces, as shown in the example presented in Figure 39 [247].

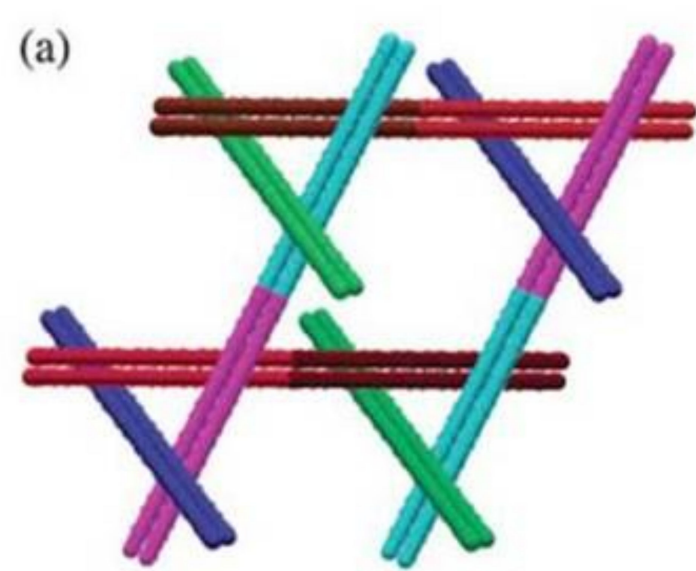

(b)

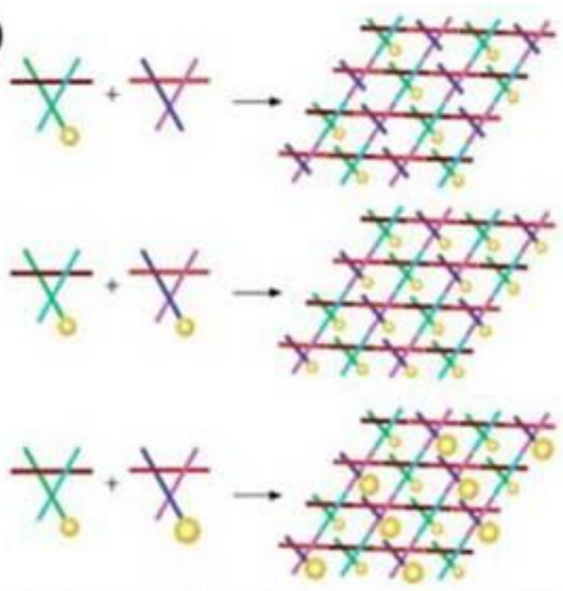

(c)

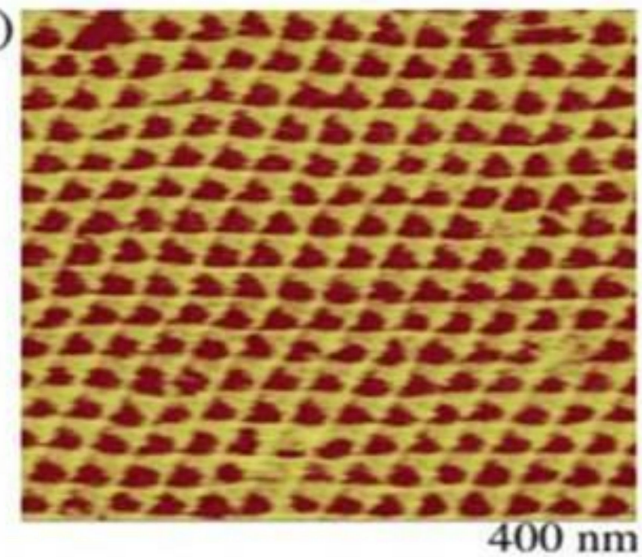

(d)

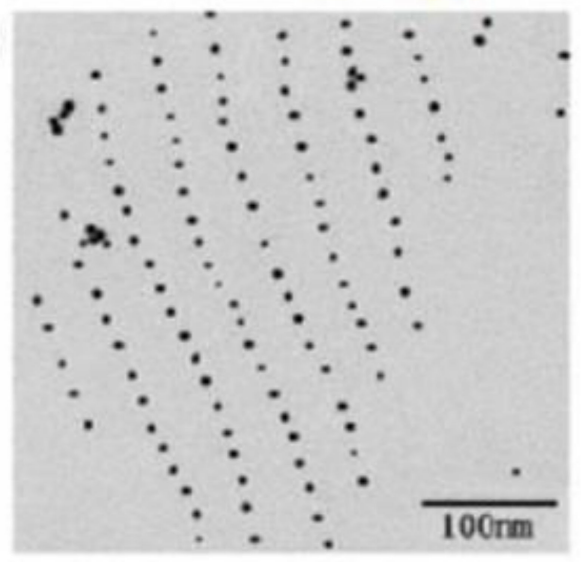

Figure 39. (a) The Au NPs can be shaped in a standard array of Au-nPs in a dx DNA template as shown by DX DNA triangles with sticky extremities, to allow the tiling of triangles; (b) Non-all ends of DNA are needed for tile purpose and the free ends could be attached to Au NP's (yellow) 5 or $10 \mathrm{~nm}$ diameter; (c) The AFM imagery on a dx-array without the adding Au demonstrate its ordinary structure. This picture only displays au-NPs. (a-d) with reference authorization, ACS, Copyright 2006 [231].

At the 5" end of one of the DNA strands, Thiol groups can be inserted into each triangle and provide for a decorated au-nP array with a $5 \mathrm{~nm}$ Au NP connection [231] Subsequently, the DNA strands modified were used in 2D DNA collections to make the Au NP "wire" lines. [248]. Four DNA strands [249] can be used to create DNA pyramid. Nanotechnology may also speak of this: the form of small, three-dimensional structures consisting of cubes [250], a bipyramid [251] and octahedrals from different strands of DNA [252] have been created. The vertices of pyramidal DNA nanostructures have been connected to Au NP (5 nm in diameter) (see Figure 40) [250].

The discovery of DNA origami was also revolutionary [253], which enabled reasonably simple fabrication of custom 2D and 3D structures, such as boxes $[254,255]$. The preparation of a DNA origami-rod and thiol-groups at specific positions on surface in order to achieve an $\mathrm{Au} \mathrm{NP}$ at the points on the spinal cord of a helix on rods surface is one of the most interesting consequences of the ability of DNA to organize Au NPs into structures of demanding physical and chemical characteristics. The chiral plasmonic structures were described previously (see Figure 41) [256]. 
(a)

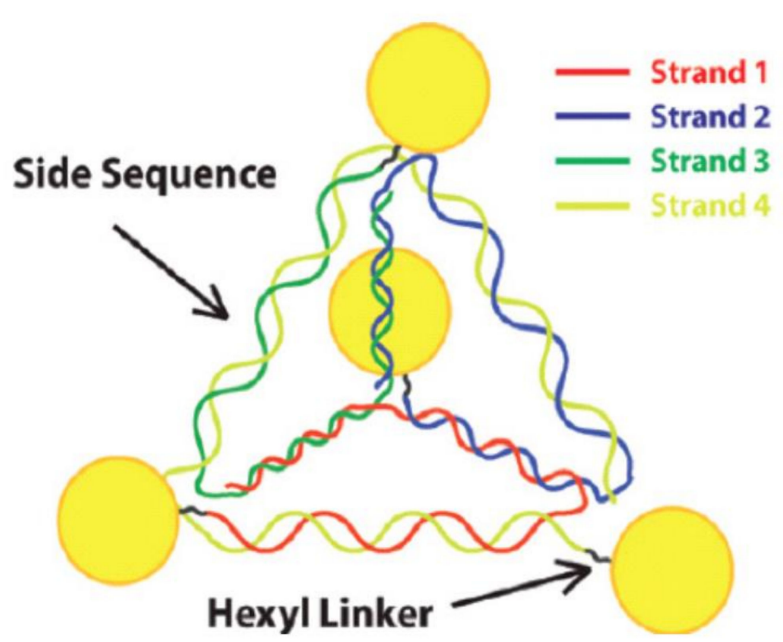

(b)



Figure 40. (a) The Free-DNA ends at every point of reference could be altered to include GNP with a linker sequence; (b) a single DNA pyramid Au NP microgram with four Au NPs visible in black circles: Reprinted with reference authorisation [249], Copyright 2009, ACS.

a



Figure 41. (a) A rigid DNA origami bundle composed of 24 parallel double helices. Gold nanoparticles are arranged in a secondary left-handed helix on the DNA origami structure. Zoom in: $10 \mathrm{~nm}$ gold nanoparticle functionalized with thiolated ssDNA hybridized to the DNA origami $24 \mathrm{HB}$. The origami structure is functionalized with biotin groups (green) on one end for the attachment to a BSA-biotin-neutravidin-coated surface (red, green and grey). (b) Transmission electron microscopy image of a nanohelix adsorbed non-specifically to a carbon-coated grid. Scale bar, $50 \mathrm{~nm}$. Reprinted with reference authorisation [256], Copyright 2013, Macmillan Publishers Ltd. 


\subsection{Interaction of Proteins}

The Au NPs have been added to proteins, such as prion proteins, virus capsides and chaperonins, for example, palladium [257] and iridium [258], for catalysts of di-chromate, decrease, and water oxidation was confirmed for catalyzes other metals than Au deposited on viral models. For prions, the saccharomyces cerevisiae Sup35p protein area of the N-terminal middle (NM) region was utilized [259]. This protein produces self-assembled amyloid fibers (about $10 \mathrm{~nm}$ in diameter) [260]. By working on the assembly conditions, the length of the fibers can be roughly regulated. If amino acids 184 of NM are mutated into cysteine, a thioaurate bond built between the $1.4 \mathrm{~nm}$ Au NP diameter and the cysteine side chain sulfur is the template for Au nanowire growth. In the expected development of a solid Au nano-wire [259], the resulting "enhancement" of the deposited Au NPs is achieved. The capsid virus is another protein template. The capsids could offer beneficial hollow nano-shells with a broad range of uses [261]. For instance, cow pea mosaic virus (CPMV), a 60 identical protein icosahedral virus [262]. CPMV was shown to be a useful breeding grout for catalysts: it was decorated accordingly with the addition of surface carboxylated or amine-groups in redox active methyl (aminopropyl) violonics [263] or ferrocene moieties [264] through the addition of surface carboxylated or amine-groups, correspondingly. The resultant particles demonstrate the capacity of electrocatalytics to be applied [262].

The addition of the Au NPs to the outside of the CPMV capsid was reported by Blum and colleagues [265]. The remains of the cysteine can be fed on the outer surface and cysteines may be inserted anywhere they are appropriate and substituted at 60 symmetrie-equivalent places with the protein structure. This virus lacks the natural surface cysteine [265]. The selected sites were devoted to three separate sites of cysteines in order to have a varied number of particles and a changing interparticle gap between the attached Au NPs and 5 to $10 \mathrm{~nm}$ Au NPs (see Figure 42).
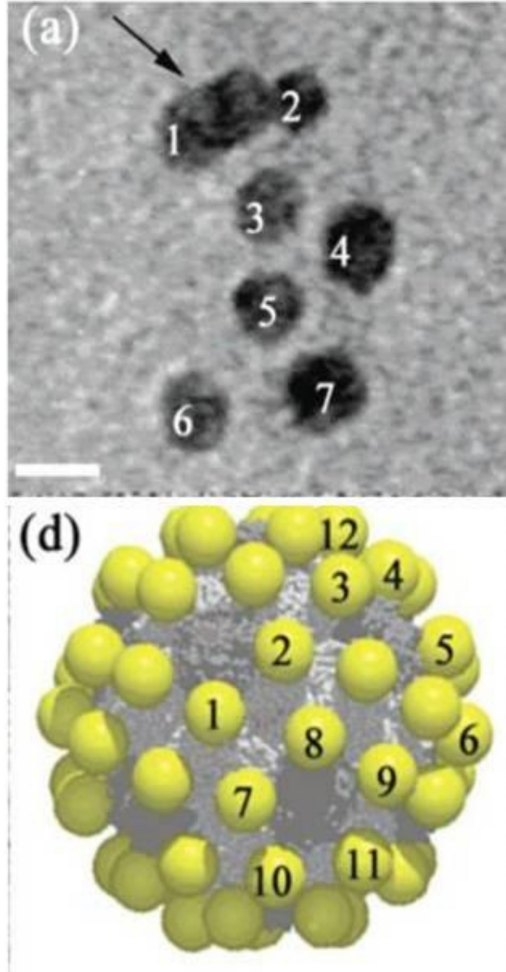
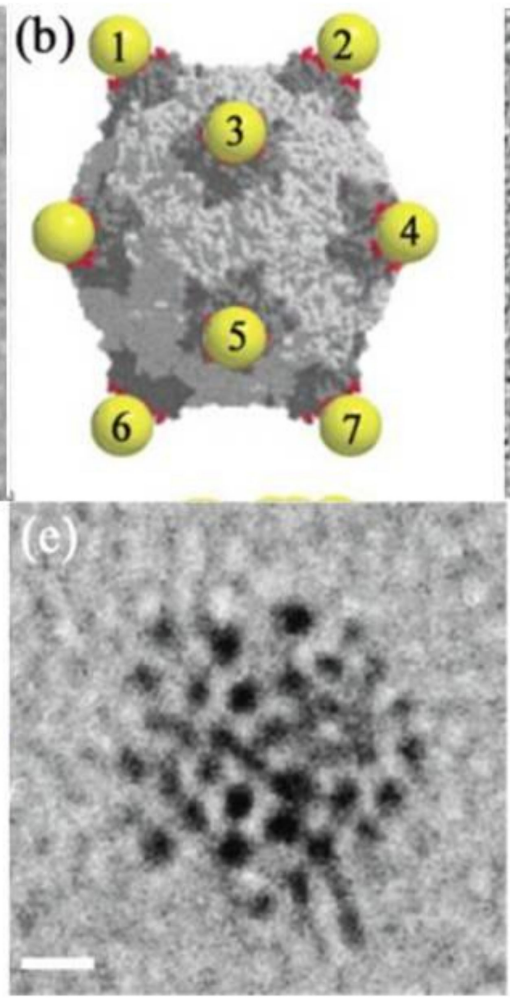
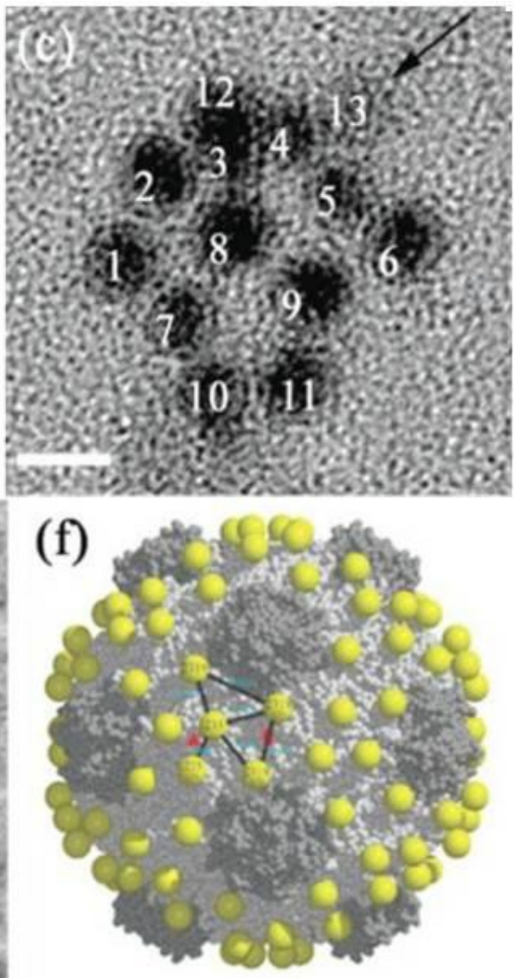

Figure 42. The Au NPs can be changed to the Cowpea Mosaic Virus (CPMV). (a,c,e) show untouched Au NP TEMs bound to various cysteine CPMV mutants with black circle au-NPs. The size of bars is $5 \mathrm{~nm}$, with the Au NP models bound to modified sites (b,d,f) the CPMV models. Reprinted with reference authorisation [265], Copyright 2004, ACS. 
Some researchers report that such virus capsids have a tremendously valuable application as catalysts [265]. In this way, they have been decorated. CPMV shows additional surface changes allowing the attachment of nanoparticles of different metals and $\mathrm{Au}$ NPs, which lead to the development of finely regulated, highly appreciated bimetallic or multi-component catalysts [266]. Tobacco mosaic (TMV) is commonly used in bionanoscience [267] and coated on the $\mathrm{Fe}_{2} \mathrm{O}_{3}, \mathrm{SiO}_{2}, \mathrm{PbS}$ and $\mathrm{CdS}$ [268] and $\mathrm{TiO}_{2}$ [269] by a large volume of metals. The use of genetically modified viruses $[267,270]$ also uses $\mathrm{Ag}$, $\mathrm{Pd}$ and Pt nanoparticles [270-272]. Newly introduced is the attachment of Au NP (6 nm diameter) to un-modified TMV in high density aqueous solution [273]. The application of TMV decorated with nanoparticles as catalysts have been shown via the creation of NPs from palladium on the TMV surface that revealed the reduction of di-chromate to catalyse catalysis of these other materials has so far been covered by Au-coated virus particles. Protein crystals, consisting of cross-linked [274] and non-circulated lysozyme, were used to template the $\mathrm{Au}$ NPs. Finally, when lysozyme crystals are grown in $\mathrm{ClAuS}\left(\mathrm{CH}_{2} \mathrm{CH}_{2} \mathrm{OH}\right)_{2}$, they cause Au-to NP's form in situ through a disproportionate reaction in protein crystals [275]. In the absence of protein, growth is much slower than the development of $\mathrm{Au}$ NPs and allows for fine controls of Au NPs up to a limit of $20 \mathrm{~nm}$. In consequence, the Au NPs made up of lysozyme crystals have shown the ability to catalyze p-nitro-penol reduction to p-amino-phenol [276].

J.B. Philip et al. [277] confirmed that protein scaffolds are used for the standard array of Au nps with chaperonin protein HSP60. The TF55 $\beta$ subunits were used in this process for sulfolobus shibatae. The two-ring proteins contain 18 subunits of proteins which can be classified as $2 \mathrm{D}$ crystals $[278,279]$. This is the structure of the double ring. The solitary native-cysteine found in protein was isolated and the structural data were used to allocate the cysteine around the protein's main cavity. To make the opening wider, residues were loosened). Furthermore, 2D crystalline Au NP collections were achieved by initially making 2D protein groups and then adding Au NPs into a solution, or initially binding Au NPs into proteins regulated by surface deposition (see Figure 43).

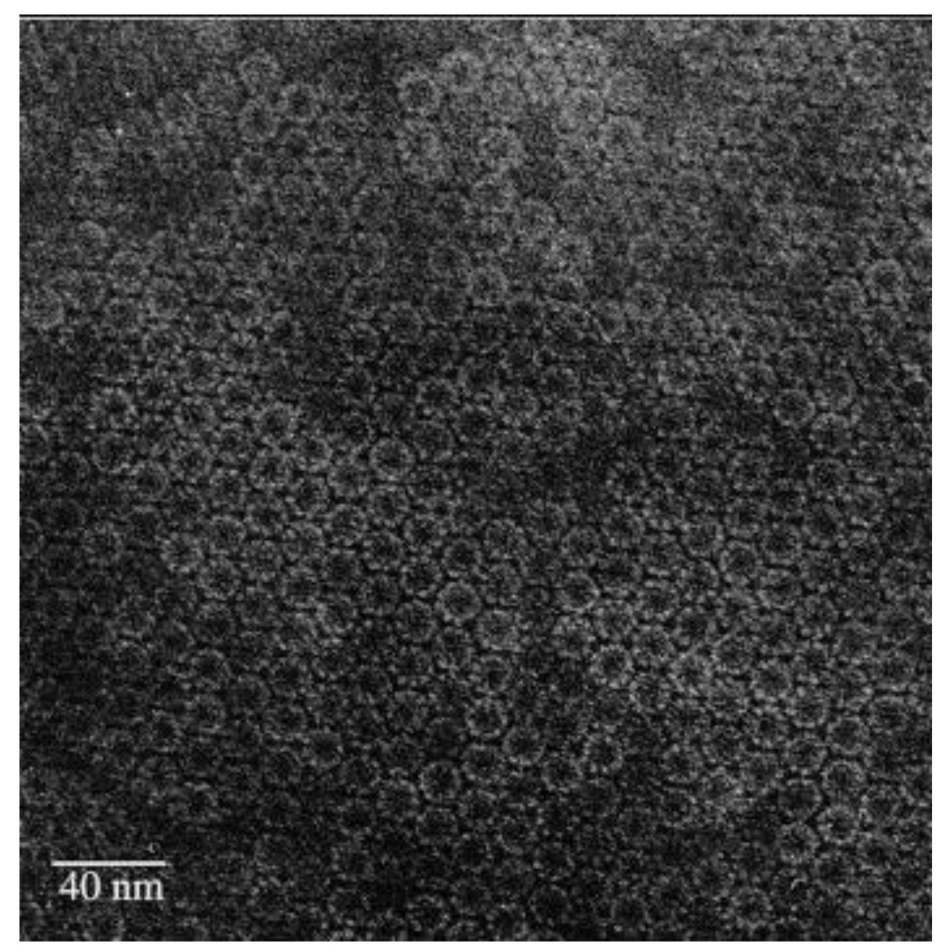

Figure 43. A negatively stained crystalline area of TF55 $\beta$-subunits reconstituted into oligomers. Reprinted with permission from Elsevier, 1998 [277]. 


\section{Catalysis via Biomolecules Anchored Au NPs}

In various reactions, $\mathrm{Au}$ NPs are being used as a catalyst every day. However, it is less understood that $\mathrm{Au}$ NPs are used to catalyze bio-molecular reactions. Two groups shall be considered in this section: first, Au NP catalysis, and second, Au NP ligand catalysis.

\subsection{Catalysis via the Au NPs Itself}

The standard Au NPs catalyzed processes is brought about by the Au NPs themselves being frequently deposited on an oxide; surface characteristics are considered. The provided illustrations clarify how the Au NPs bring the catalysis of biomolecules. Haruta first put forward Au NPs' catalytic capacities, in which Au NPs induced carbon monoxide oxidation. In the following years, Au NPs catalyzed several non-biological reactions (i.e., see Bond et al. [280], Hashmi et al. [281] and Pina et al. [282], for critical reviews). Many of the major characteristics for Au NP catalysis, including: (1) low coordination, are envisaged or understood; by theoretical modeling, Mills and colleagues [283] showed that a small $\mathrm{Au}$ group or irregular Au surfaces are catalytic, since they confine lone $\mathrm{Au}$ atoms with little organization, such as HOMOs, that project into space, rather than relocate; they suggested that it would allow load transfer to the $\mathrm{O}_{2} \pi^{*}$ orbital. (2) Size: size is key, since Au NPs with lower catalytic size are going to have a high catalytic rate [284]; this pattern is not unexpected, since only surface atoms are catalyzed, and given (1), a higher degree of low atoms of coordination across boundaries and deficit is more likely to be present in smaller groups. Furthermore, certain geometries and groups tend to have unique characteristics called "magical number" [285], and particles like Au55 appeared to be closed shelled groups and extremely oxidation impermeable and thus are strong catalysts for oxidation [286]. (3) Scaffold interface: the existence and effect of the interface between the deux is usually examined by the Au NP on an oxide support. The supporting point at which the Au NPs occur was proposed as the oxidation centre. The smaller $\mathrm{Au}$ NPs are more involved due to the greater amount of their support system. The Au which is in contact with the help in the event of $\mathrm{CO}$ oxidation is predicted to be $\mathrm{Au}^{3+}$ and the active site for dioxygen activation is suggested by the cationic $\mathrm{Au}$ [287]. The scaffold is a life molecule like the virus capsid or DNA that is considered in that discussion to have an effect on Au catalytic action. In spite of this, enzyme-catalysts illustrate us that the exact arrangement of atoms and remains is necessary to construct an active-site [288] and sensible to think that the regulated, accurate posing of Au NPs in three dimensional associations can be used for the next catalytic system. When measuring large numbers of Au NPs on the capsid with a high density, supporting catalytic possessions would probably be taken. Obviously, biological molecules usually exist in aqueous solution. In solution, Au NPs often somehow can conduct catalytic tests free of charge [289]. These processes are less well studied, but may be more suitable for biocompatibility issues. (4) Adsorption mode: According to empirical research [290], the adsorption activation on the surface of the Au NPs is carried out by three modes, i.e., end-on, top/bridge/top and bridge/bridge, the strictly activating $\mathrm{O}_{2}$ is better separated with the bridge-bridge surface of the Au NPs (see Della Pina et al. for an overview [282]). There are several examples where the effect of proteins in relation to biological properties was imitated by inorganic nanoparticles [291]. This also involves enzyme catalysis imitation. The glucose oxidase act was copied with $\mathrm{Au}$ NPs (GOx). The GOx is an enzyme that catalyze glucose oxidation into glucolactone, creating $\mathrm{H}_{2} \mathrm{O}_{2}$ simultaneously (see Figure 44A) [292]. This result provides the foundation for numerous blood glucose-meters used by the diabetes patients. In 2000, diabetes was projected to hit $2.8 \%$ by 2030 [293], i.e., an increase from 171 million to 366 million people, and the figure was expected to reach $4.4 \%$ in 2000 . Diabetes is serious, progressively difficult condition. The condition is characterized as high blood sugar either because of inadequate development of insulin (type 1) or because the body is unable to properly use insulin (Type 2). The disease can result in many serious and long-term problems for those it affects [291]. 
A
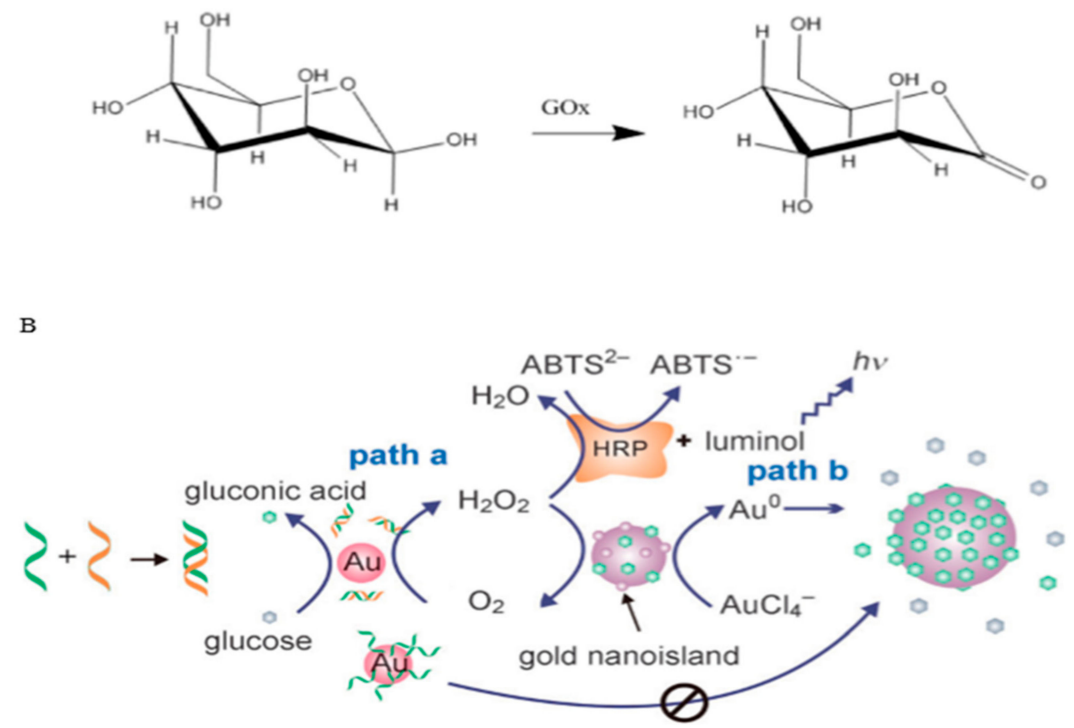

Figure 44. (A) Glucose togluconolactone oxidation catalyzed by glucose oxidase (GOx). (B) Application to the sensor to find DNA hybridization of GOx-like catalytic activity of Au NPs. The Au NPs (red) will catalyze glucose oxidation and produce peroxide under the present pattern. This can be used in the subsequent oxidation of ABTS in a horseradish peroxidose (HRP) colour (path A). Peroxide production also catalyzes $\mathrm{AuCl}_{4}$ to $\mathrm{Au}^{0}$ reduction, which increases the size of Au NPs (path b). (A) Replicated with authorisation from reference [293] Copyright The Biochemical Society. (B) Reproduced with permission from reference [294], Copyright 2011, WILEY-VCH.

Instead of being able to display blood glucose levels precisely, insulin is a real action for diabetes, primarily Type 1 . A characteristic control technique uses GOx for the oxidation of a limited amount of blood. The enzyme is renewed with the oxidation of a moderator to assign the electrons to the cell electrode, thereby producing current. The emitted current size is relative to the glucose sample amount. These machine-based sensors have been extremely successful [295]. Au NPs are well-known to be useful in the equation of glucose, gluconic acid and $\mathrm{H}_{2} \mathrm{O}_{2}$ to catalyze the same oxidation reaction as shown in (1) [296].

$$
\mathrm{O}_{2}+\text { Glucose } \rightarrow \mathrm{H}_{2} \mathrm{O}_{2}+\cdots \text { gluconic acid }
$$

In solution and solid, Au NPs were considered as active catalysts for glucose oxidation. In a solution, an excess catalytic rate of glucose transfer to gluconate was found to be shown in the Au NPs, because the particle's diameter was reduced, up to the lowest $(3.6 \mathrm{~nm})$ diameter measured. Even though catalytic activity was still below the marketable enzyme system [297], it can still be assessed that if smaller particles are used, the rate will progress. Luo et al. [298] further investigated the catalysis and showed that passivation of the surface of Au NPs suppressed the catalyst and associated the role of a surface Au atom. The catalytic activity showed a Km-length of $6.97 \mathrm{mM}$ (comparing GOx's $4.87 \mathrm{mM}$ ) with Michaelis-Menten, whereas the Kcat was twice that much higher with the Au NPs. There was no effect on glucose oxidation in the research of other metal nanoparticles. The authors showed that catalysis of Au NPs was dependent on size, wider $\mathrm{pH}$ and temperature ranges than that of GOx and that this was obviously possible as a powerful part of glucose control devices. In DNA hybridization, the capability of Au NPs to catalyze the oxidation of glucose was used (see Figure 44B) [294]. ABTS2 - (2,2'-azino-bis(3-ethylbenzothiazoline 6-sulfonic acid) otoxides the peroxide produced by the catalytic effects of the Au NPs along with horseback peroxidase (HRP). These consequences are of a blue color [294]. (ds)-DNA is not closely linked to the Au NPs, so the processes do not have a major consequence. However, the single strand DNA exhibits substantial binding, resultant in passivation of the surface resulting in surface passivation. The machine will then use colorimétric 
exams or direct remarks on the size of Au-and/or NP's the plasmonic effects for free DNA or RNA strands (i.e., disease-connected miRNAs) that arose because of the fact that the peroxide prooduced could be employed to lessen $\mathrm{AuCl}_{4}$ to $\mathrm{Au}^{0}$, Au NPs producing enhance particle size, a procedure repressed by attached ssDNA. The last case is the catalytic $\mathrm{Au}$ NPs of biological molecule structural variations. Au NPs tend to catalyze protein reshaping in this situation. The fact that Au NPs can mix with proteins and bring structural variations is well known. Such discrepancies are usually the result of structural and functional loss due to protein denaturation or development of the corona protein acquired around the Au NPs [299-301]. The development of higher-order structures induced by $\mathrm{Au}$ NPs is reduced. In Bacillus species, TRAP is a toroidal protein [302]; the proteinTRAP (trp-RNA binding shrinking protein) was employed. In Bacillus species, TRAP is a toroidal protein. In vivo, TRAP participates in the reaction regulation of the preparation of tryptophan. The crystal structures of both the B TRAPs, stearothermophilus [303], and the B. subtilis [304] were recognized and showed that TRAP has a diameter of about $8 \mathrm{nms}$ and consist of eleven equal monomers each with approximately $8.4 \mathrm{kDa}$. The protein has other features beneficial to bionanosciences because of the exciting and well-defined form. These include high thermostability [305] and the ability to withstand multiple mutations of the surface without affecting overall structure. Up to now, TRAP has been used as part of the floating nanodot gate transistor to generate symmetry-changed structures and to create an automatically assembled nanotube protein [306] as a constituent of a floating nano-dot gate-transistor [307]. The mutation of the lysins at position 35 (37 in B. stearothermophoilus TRAP) to cystein results in the interaction of TRAPs with Au NPs (see Figure 45). It has an ability to tie strongly to $\mathrm{Au}$, because of the cysteine sulfhydryl group. However, the wild protein does not contain cysteines. The TRAP's residue lysine 35 is accessible to the surface and lies in the outer rim of the ring unprotected and a motivating conformative transformation is perceived when mutated into a cysteine and blended to $1.4 \mathrm{~nm}$ Au NPs.

Owing to the unavailability of Au NP, the transmission-electron-microscopy (TEM) perceived TRAP protein appear to be negligible from the non-modified protein, i.e., it looked about $8 \mathrm{~nm}$ in diameter as a small donut-shaped ring (see Figure 45). No important consequence was observed in $1.4 \mathrm{~nm}$ Au NPs applied to the wild type protein. However, the incorporation in the mutant protein of the same Au NPs affected a drastic shift. The mutant-protein sample had no proof of the unique ring-shaped protein after several hours of production at $4{ }^{\circ} \mathrm{C}$ and was replaced with large circular proteins of almost $20 \mathrm{~nm}$ in diameter. Study with cryo-electron tomography of these particles revealed that the produced structures were essentially spherical hollow proteins. The spheres looked like two distinct dimensions (diameter about $17 \mathrm{~nm}$ and $21 \mathrm{~nm}$ ), both depending on the Au concentration (with the superior proteins prevailing at low Au NPs concentration). The similarity between these hollow-formed particles and the governing viral capsids is known as "sphere like capsid" [308]. In addition, although there is sometimes a small cluster of one or more Au NPs inside a protein shell in one location, in other situations, no $\mathrm{Au}$ particles can be presumed to be linked with the shells (though it should be well-known that its difficult to observe $1.4 \mathrm{~nm}$ Au NPs under the TEM). All of a sudden, this implied $\mathrm{Au}$ could behave catalytically. It is definitely important to see how the Au can function through simple templating or gagging effects because it is the divergence between the size of the Au NPs and the following protein shell. The theory of catalysis is maintained by the fact that the same protein remodeling is possible even at low concentrations of $\mathrm{Au}$ NPs. Although the catalytic hypothesis is intriguing, now it is unconfirmed that it is difficult to fully align the small size of Au NPs in the shaped CLSs. Moreover, even though significant numbers of Au NPs tend to lack the CLSs, Au atoms have not finally been lined up. There were intriguing clues from the reaction path [308]. If the reaction is discontinued and seen under TEM, a minute after the Au NPs initiation, numerous stable TRAP rings were extinct before but few CLS are produced and several thread structures appear to be unfolding or disassembling protein in place a large number of thread-like structures. The Au NPs can constitute an infringement of the stable TRAP ring separately 
and (partly) unfoldment in the capsid method of monomer proteins, as previously reported. The processes by which this can happen are still unclear. The exact role of Au NPs in the processing of CLS waste is clearly illuminated. It is strongly suggested that interaction with Au surface is necessary if the reactions only occur in the TRAP cysteine mutant; it is not pure if it is direct, or mediated by oxygen as defined in other catalytic oxidative reactions of Au NPs [309]. Au adatoms on the surface of the Au NPs might be the site of Au-S bond improvement. The Au-S bond improvement site may be gold adatoms on the surface of the Au NPs. The structure of the au-thiol binding has recently been studied and the nature of au-thiol effects has been analyzed remarkably and has been purified and much remains to be agreed upon [310]. The re-modification of TRAP into the CLS is important because, to date, protein transmission with Au-is NP's generally non catalytic and non specific, i.e., it is common to denaturate protein [311-313] or to form a "corona" in Au NPs [314]. Even though ability to reshake proteins is interesting in certain cases, it must be understood that a great deal of work residues to approve is required if the functional outcome is actually catalytic and specifics the mechanism. If this result of protein re-modeling is adequate, then this type of reaction to Au NPs may become a beneficial tool in bio-nanotechnology.

a
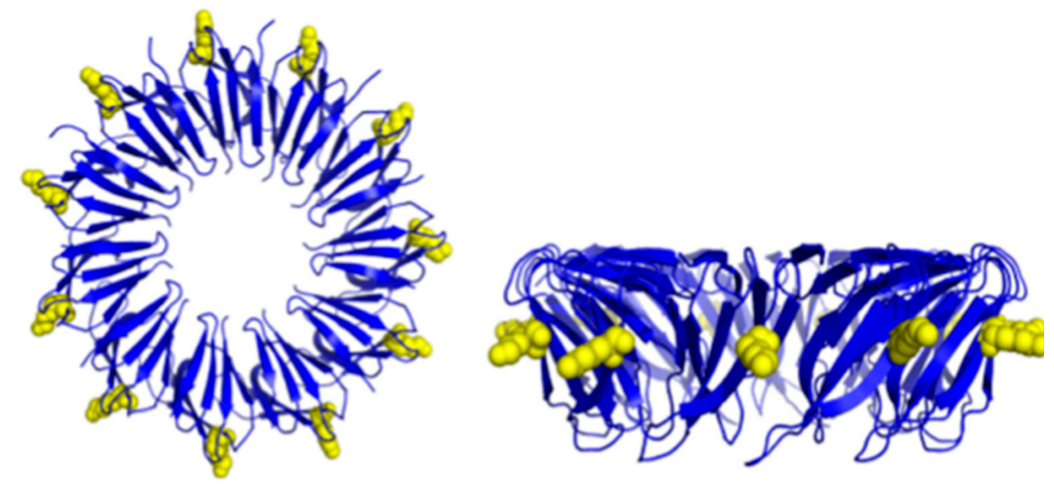

b
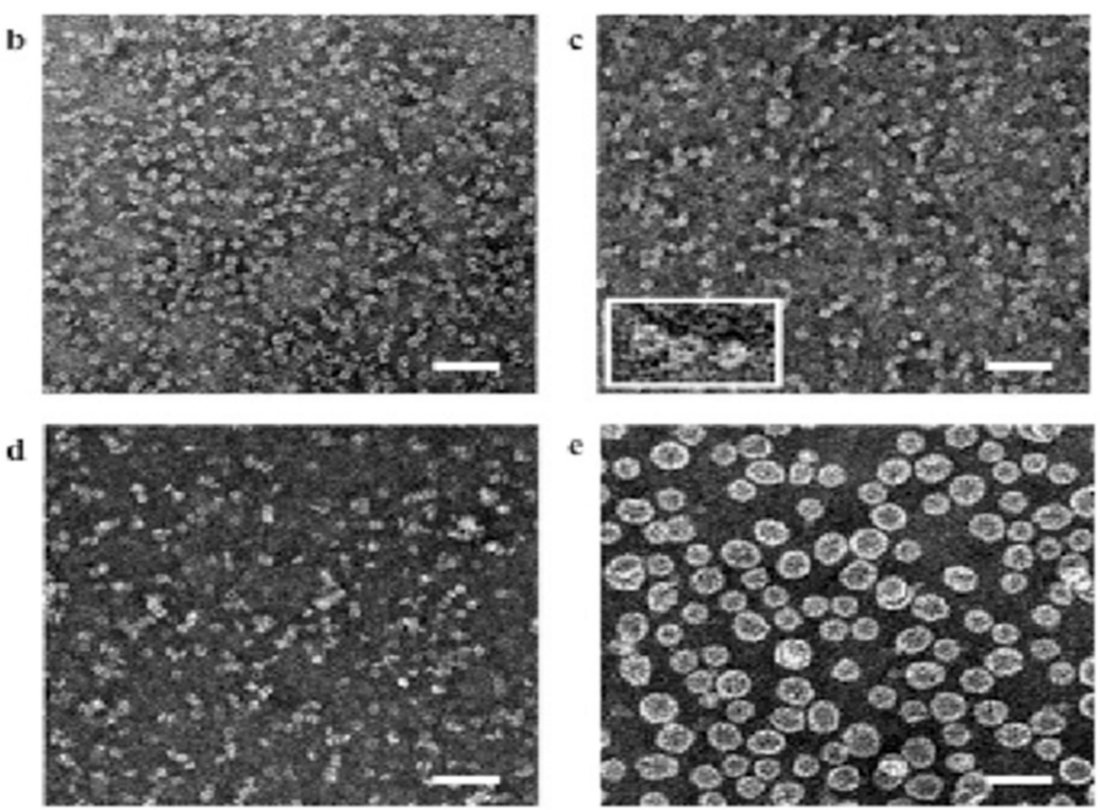

Figure 45. (a) The TRAP crystal structure (pdb 1qaw) revealed in two equally orthogonal views. Reprinted with permission from reference [303] Copyright 1999, Elsevier. The protein with residue at site 35 highlighted in yellow space filling as represented by the cartoon format; (b) TEM micrograph of the purified wild-type TRAP; (c) TEM micrograph of the purified cysteine-mutant TRAP; (d) TEM micrograph of the wild-type TRAP in the Au NPs presence; (e) TEM micrograph of the cysteine-mutant TRAP in the Au NPs presence. Note the form of bulky structures. Scale bars $=40 \mathrm{~nm}$. (b-e) Reprinted with permission from reference [307], Copyright 2012, ACS. 


\subsection{Catalysis via Anchoring Ligands}

The ligands are the functional groups that have been bound to the au-NPs for catalysis. In these reactions, the ligands bound to the Au NPs surface are the active groups responsible for catalysis. The advantage of using Au is that it is simple to keep and just has the advantage of being able to provide a monolayer of a catalytic ligand with high density. A wide variety of ligand monolayer skills have been investigated in Au NPs [315,316]. Biological mechanisms naturally occur in aqueous solutions and thus Au NPs will touch those using Au-colloids in solution without assistance. Fortunately, in a number of processes such Au NPs demonstrate catalytic activity [317]. The breakup of the phosphate link [318] was the catalytic reaction in one case. An in vivo reaction that may be caused by enzymes like restriction enzymes, a reaction which, in vivo, can be brought about by enzymes such as restriction enzymes [319] and topoisomerases [320]. The Au NPs were shaped and coated with a functionally azacrown thiol with a diameter of almost $2.5 \mathrm{~nm}$. This is confined triazacyclonans that can bind Zn-component metals (II). Initially, a hydrolysis reaction was observed, rather than RNA, by using 2-hydroxypropylp-nitrophenyl phosphate (HPNP). The second order HPNP rupture constant was found to be more than 600 times larger than the Au NP rupture. This was understood in part by the influence of indigenous concentrations and the stability of the transitional state. It was also found that the shaped supramolecule would catalyze the same break-up of actual RNA dinucleotides. In addition, extreme kcats and KM values of $6.7 \times 10^{-3} \mathrm{~s}^{-1}$ and $3.1 \times 10^{-4} \mathrm{M}$ were found to be more detailed [321] using triazacyclonane. $\mathrm{Zn}$ (II) as a catalytic unit along with unreacting ligands, respectively. When the mole portion of the catalytic unit was 0.4 of all ligands, the extreme Kcat value was obtained. Theoretical intentions indicate that lonely catalytic units were not slower, and all catalytic units worked in unison. Another example was the formation of ornamented esterase Au NPs [316]. The Au NP scaffold here allows the dipeptide functionality to be attached. In the case of an imidazole or carboxylate group acting as a typical foundation and ordinary acid in the reaction, several copies of histidine and phenylalanine, consisting from dipeptide moiety $\left(\mathrm{HS}-\left(\mathrm{CH}_{2}\right)_{11} \mathrm{CO}-\mathrm{HIsPhe}-\mathrm{OH}\right)$, were involved on the surface of $\mathrm{Au} \mathrm{NP}$, to activate the active site of esterase that is designated to produce the two amino acids [322]. Besides 2, 4-dinitrophenyl butanoate (DNPB) and Z-leucin-p-nitrophenyl ester activated esters, improved particles have been confirmed (Z-Leu-PNP). The results suggested that the covered Au NP was more successful than the no $\mathrm{Au} \mathrm{NP}$ controls, primarily at a low $\mathrm{pH}$, because of the imprisonment on the Au NP surface [316]. The impact was recognized as cooperative. The DNA pinching catalysis was identified by Hsu et al. Hsu et al. [323] Au NPs were again split in the ability to limit ligands to nearby high concentrations. Arylhydrazones were, in this case, bound approximately $13 \mathrm{~nm}$ in diameter on the Au NP surface. These ligands can chip DNA after UV light ( $312 \mathrm{~nm}$ ) has been disclosed and pinnacles have improved compared to the lack of Au NP [324] due to the concentrations of these ligands at local level as a result of prison on the surface of the au-NPs. In other cases, a DNA phosphodiester hydrolysis was obtained by adding BAPA (bis-(2-aminopyridinyl-6-methyl)amine) Zne(II) complexes to a surface approximately $1.8 \mathrm{~nm}$ of diameter Au NP [325]. In this case, a bimetallic site was given with a ligand clustering in combination with a hydrogen bond framework to break through the activated zinc lewis acid (see Figure 46). This has resulted in being 100 times largerthat of the ligand in the absence of Au NPs by breaking down the DNA-model substratum (bis-p-nitrophenyl phosphate, BNP). The splitting of the DNA substratum of the plasmid was also perceived, which, remarkably, resulted in the development of linear DNA products due to numerous break-up reactions [326]. Catalysis has also been confirmed of peptide-based reactions used. Fillon et al. [326] used trimethylammonium-functioning $\mathrm{Au}$ NPs that produced a positively loaded surface. Peptides with negative residues were intended to be in appropriate places connected to the cationic monolayer ligand. Two peptides, the five-heptad alpha helix, were used for two halves. These were intended to consist of one $\mathrm{C}$-terminal thioester and one $\mathrm{N}$-terminal cysteine in order for the two peptide half ligation to occur by indigenous chemical ligation [327]. The ligation rate was 
substantially increased in the event of Au NPs. The catalytic effect is possibly due to the reactive reactants in close proximity to the Au ligand. The peptides have been planned to be helical at acidic $\mathrm{pH}$ only, so that they can only temper and be self-replicated at acidic $\mathrm{pH}$. However, added Au NPs at neutral ph mean that both halves have helicity and have been able to generate ligation product at a neutral $\mathrm{pH}$ [326]. This finding may be of interest to researchers in abiogenesis where it is likely that an auto-replicating peptide has become the first "Darwinian Ancestor" [328].

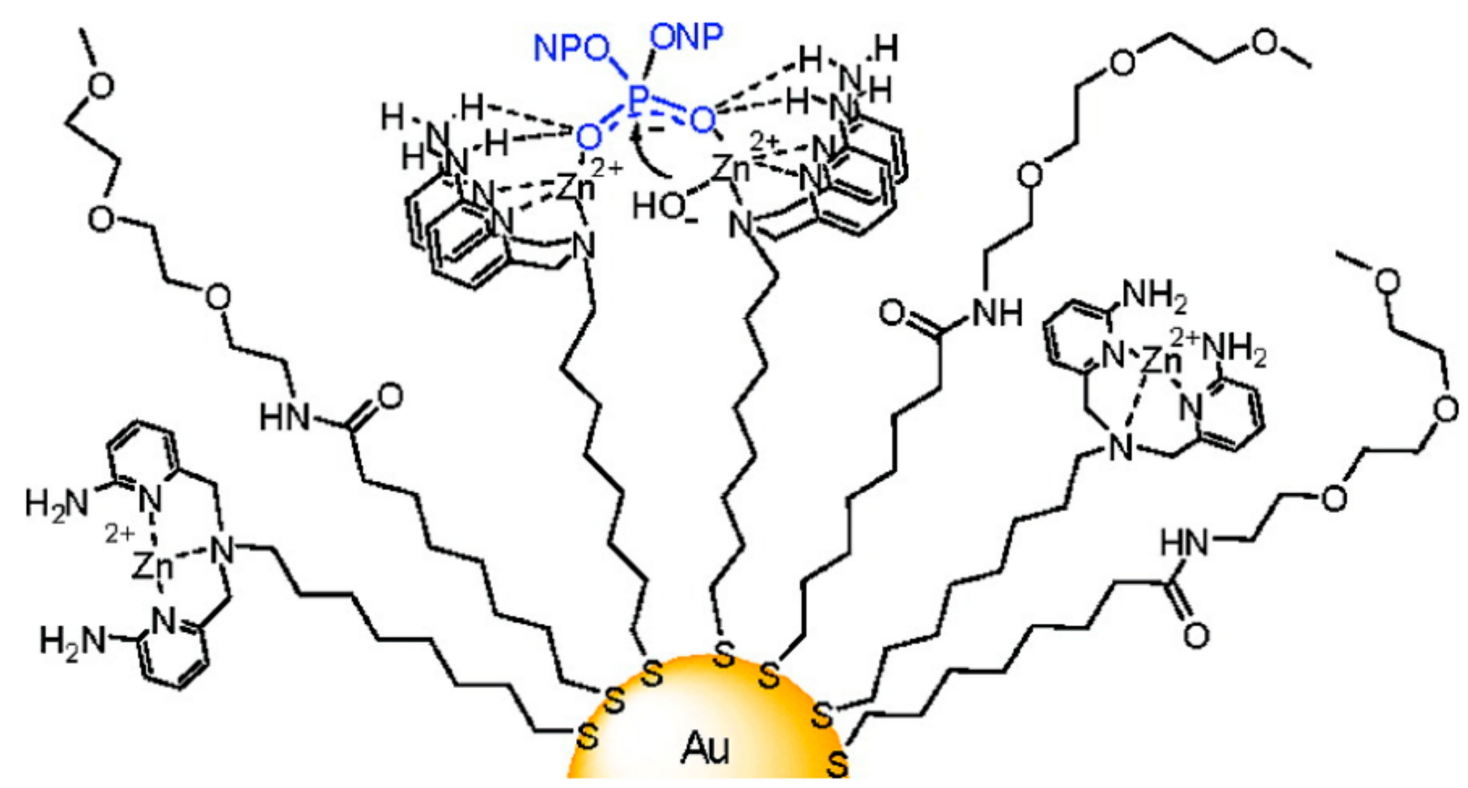

Figure 46. Bimetallic site bunching can build $\mathrm{Zn}$ (II) complexes attached to $2 \mathrm{~nm}$ diameter Au NPs. This site is demonstrated by the scission of a Bis-p-nitrophenyl phosphate DNA model substratum (BNP). Copyright 2008 ACS, reproduced with reference permission [326], Copyright 2008, ACS.

The ligand modified Au NPs have been used in sensors with catalytic characteristics. Bonomi et al. [321,325] developed a transphophorylation method for 2-hydroXypropyl4-nitrophenylphosphate (HPNPP), covering Au NPs with triazacyclonane·Zn (II). This generates p-nitrophenol with absorption of $400 \mathrm{~nm}$ to verify its concentration. This function was broken to provide a sensor that accounts for protease activity, subtilisin A. Because of the positive ligand on the AU-NP surface, the entire oligoanionic peptide substratum was able to bind to the surface of the AU-NPs. The surface peptide stopped the HPNPP reaction. On the contrary, this binding was removed when the enzyme broke down and the reaction with HPNPP resulted in a shift in the signal of absorption. This mechanism was shown to identify the behavior of other enzymes that altered their negative charges. Attachment of the nucleic acids to Au NPs is negligible, as well as extensively recognized all DNA RNA molecules with enzyme activities (DNA zymes and Ribozymes) [329,330]. Detectors/sensors are widely used in conjunction with Au NPs. The paired series of DNA strands are a twin helix, which aggregates AU-NPs which are protected by certain arrangements and changes in color because of plasmon. The addition of DNAzymes, which split the DNA when a particular cofactor occurs, disintegrates the cofactor and creates a colorimetric detector. In this case the DNA strands were incomplete, and $\mathrm{Au}$ NPs linked into aggregates with a color of blue and a DNAzyme. DNAzyme cuts the joint strands in the presence of $\mathrm{UO}^{22+}$ leading to disaggregation of the Au NPs and a color shift to the red in a single uranyl sensor [325]. DNAzyme has been used in different circumstances in conjunction with the Au NP surface (see Liu and Lu review [331]). In comparison to this technique, Zhao et al. [332] the Au NPs were coated with DNAzyme, which created an Au NP aggregation when a cofactor occurred. In these experiments, Au NPs have been updated by inserting a surface ligand consisting of a single DNA (with a 
single RNA) strand and a DNA strand that is incompletely complementary to the " $8-17$ " DNAzyme [333]. In the presence of $\mathrm{Pb}^{2+}[166,334]$, this DNAzyme can cut DNA that contains a single RNA connection. In addition to aggregation, the DNA ligand on the $\mathrm{Au}$ NP is stabilized even at relatively large salt levels, due to the DNA's duration and negative load. The DNAzyme splits the ligand when the $\mathrm{Pb}^{2+}$ cofactor is present, thereby eliminating its stabilisation. The Au NPs then add up to $10 \mathrm{~min}$ at room temperature to change the red to purple color. Enzymes may be bound to the Au NPs as well. Brennan et al. [335] lipase from the lanuginosal thermoplasms could be fixed to an Au NPs diameter of $\sim 14 \mathrm{~nm}$. In that case, a thiolate composed of azide-terminated ligand functioned to the surface of the A-NPs. In order to supply the acetylene group for click-chemical attachment processes for Au NPs, a single available lysine residue was improved to the 30-kDa enzyme. Even if the reaction is actually catalyzed by $\mathrm{Au}$, the ligand may still play an important role because it can restrict the entry of the reactants to the Au surface, as has been shown for example in tests of the capability of Au NPs coated with altered ligands to catalyze glycerol aerobe oxidation [336,337] in large parts of the body (tetrakis hydroxypropyl phosphonium (THPC)).

\subsection{Catalysis via Functionalization of Asymmetric Oligonucleotides}

A magnetic sphere was used as a geometric restriction template for Au NPs functionalisation with two distinct forms of oligonucleotides on the spot (see Figure 47). Functions for asymmetric oligonucleotides in Au NPs were carried out through: (1) magnetic microparticles with $3^{\prime}$ thiol-finished thirty-mer oligonucleotides (MMPs), (1) 3'hydroxyl-modified "extension" oligonucleotides, (2) opposite half of the oligonocleotides in MMPs and (3) A (3) (2) A) Functionalized with iron-oxide cores) magnetic microparticles. The Au NPs and the MMPs with oligonucleotides were functionalized using standard procedures. The complex, (4), was made by connecting three entredent to the 5 -phasphate group of the $\mathrm{Au}$ NPs oligonucleotides by using a beffer ligation, which was changed to oligonucleotide by the MMP. The corresponding T4 DNA ligase was introduced in order to catalyze phosphodiestral bondage between three'-hydroxyl and five'-phosphate, applied to the Au-Np oligonucleotides. There was, therefore, a new $30 \mathrm{mi}$ oligonucleotide bond that could only be hydridized in the Au NP segment using the MMP template [338]. The complex obtained, (5), was separated through magnetic separation from the reaction mixture. The new anisotropically updated Au NPNPs, (6), were isolated from the MMP templates by means of a ligation buffer and heating.

The DNA melting tests include the full return of the enzyme ligation. The Au NPs can be isolated from the MMPs in a reversible manner, raising the temperature above the DNA connector's melting point. The melting point, Tm of the duplex DNA machining unit after ligation is estimated to be $20^{\circ} \mathrm{C}$ over the Tm duplex before the temperature binding and has been determined to be $74.5^{\circ} \mathrm{C}$ (see Figure 48A), contrasting the blue and red lines by observing extinction at $520 \mathrm{~nm}$ (plasmone resonance for Au NPs). With changes of the three strand 30 bp nick structure into a constant two strand 30 bp duplex this big increase in Tm is reliable, until melting SEM particle analysis shows that thousands of particles are committed to each MMP (see Figure 48B). The melting process is mainly characterized after the ligation phase by a sharp molting process that shows the Au NPs that have all hybridized on the MMP surfaces have been connected to the oligonucleotide extension. Dehybridisation in nanopure water, controlled for removal of the $\mathrm{mmp}$ with a magnetic separator, has led to the separation of the ligated Au NPs from the mmpp. The extended strands add asymmetry to the surface structure of the DNA-functional Au NPs, enabling a highly directional programming of the particle set through hybridisation. The 13 and $30 \mathrm{~nm}$ $\mathrm{Au}$ NPs (molar ratio 10:1) were mixed as a waterproof definition and were asymmetric with opposite delay oligonucleotides. Since the Au NPs could only hybridise with delay oligonucleotides, a "cat paw" structure has been developed. 

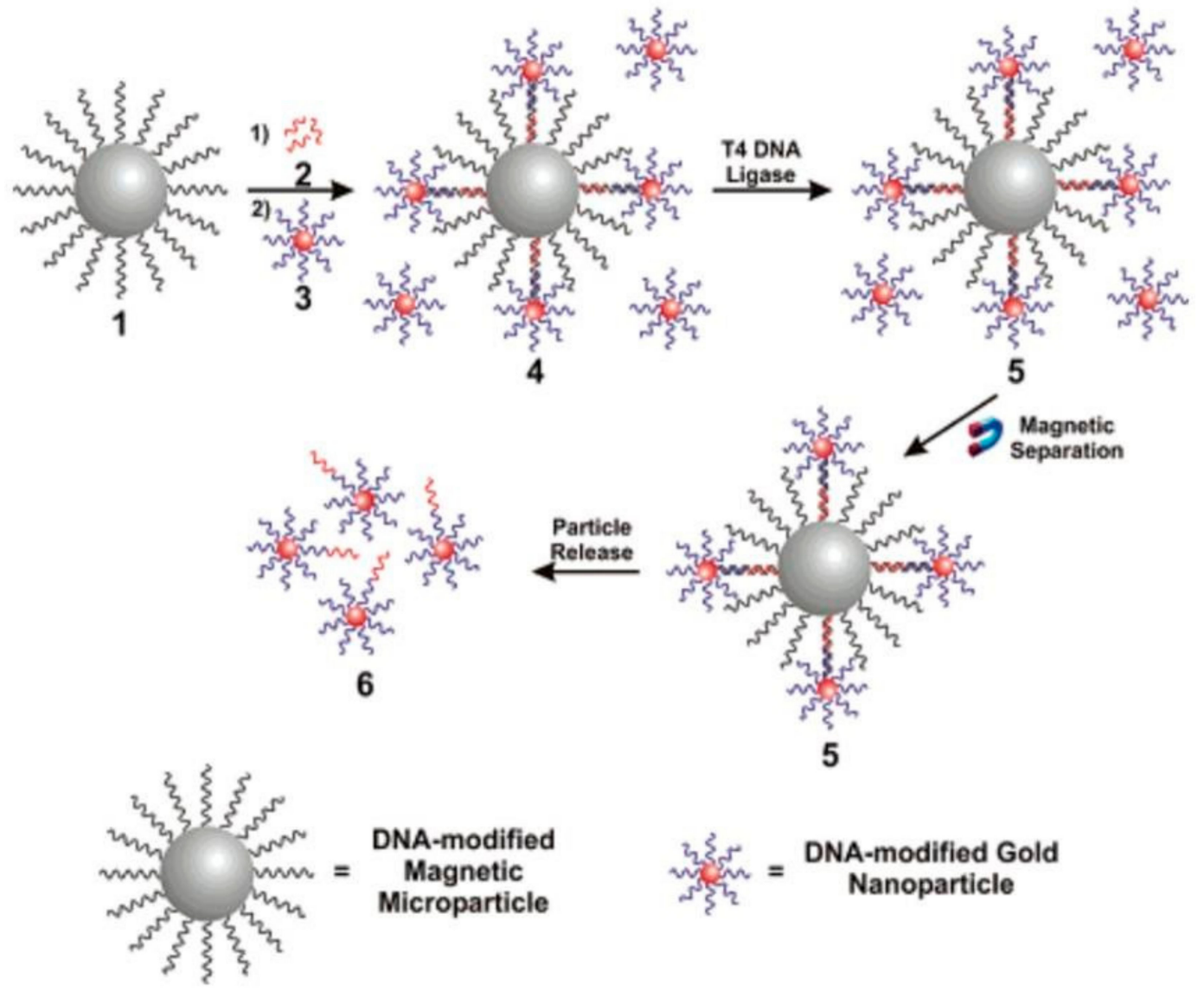

5

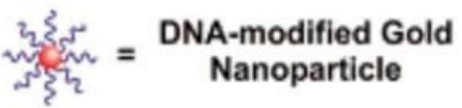

Figure 47. Functionalization of Au NPs with asymmetric oligonucleotides. Reproduced with permission from reference [339], Copyright 2005, ACS.
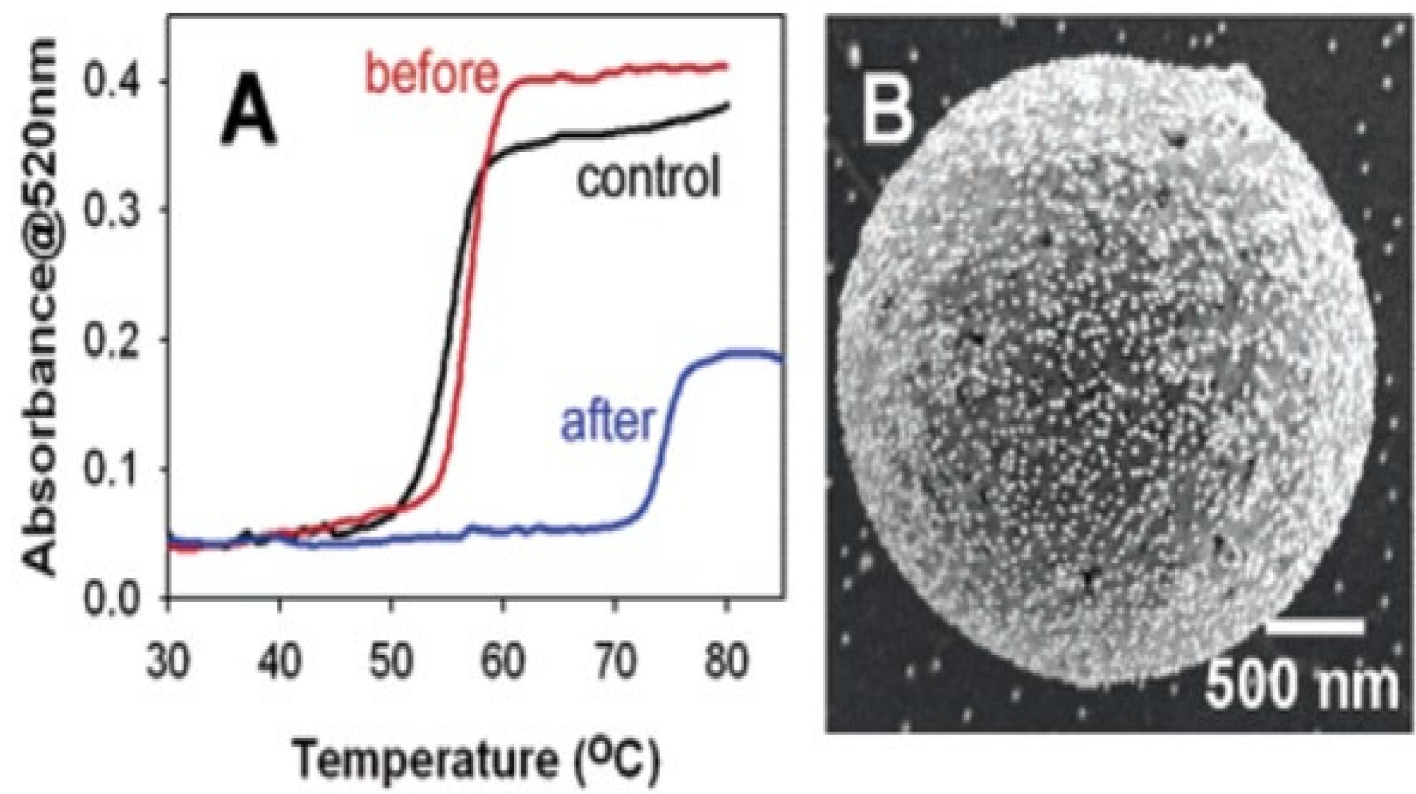

Figure 48. (A) $13 \mathrm{~nm}$ Au NP DNA melting curves hybridized by delay DNA with MMPs. The ligation stage increased dramatically, from $54{ }^{\circ} \mathrm{C}$ to $74.5^{\circ} \mathrm{C}$, before (red), after the melting temperature (blue). The ligation stage showed limited impact in addition to the extension DNA (black curve). (B) The $30 \mathrm{~nm}$ Au NP SEM picture on an MMP's surface. Reproduced with permission from reference [338], Copyright 2005, ACS.

The structures of cat paws propose that $1 / 3$ to $1 / 2$ of the surface of each Au NP of $30 \mathrm{~nm}$ should be asymmetrically controlled with oligonucleotide delay (see Figure 49A,B). 
We react asymmetrically to $13 \mathrm{~nm}$ Au NPs with $30 \mathrm{~nm}$ Au nPs, which are functionalised with the oligonucleotides opposite only to the prolonged $13 \mathrm{~nm}$ Au NPs to further demonstrate the capabilities of this association scheme. The asymmetrical functions of the Au NPNPs, (6), resulted in a settled satellite structure of one $30 \mathrm{~nm}$ Au NPs with an area of $13 \mathrm{~nm} \mathrm{Au}$ NPs not being agregated, but shaped instead. TEM sample analysis reveals that Au NPs is hybridized with six to $1013 \mathrm{~nm}$ Au NPs at almost every $30 \mathrm{~nm}$. Aeration of the sample and the electron beam will significantly distract the DNA duplex state; however, TEM data show that the asymmetry of (6) in the satellite-structure system prevents the sequential oligomerization process. In particular, the complexes in satellite-like nanoparticles are $6 \mathrm{~nm}$ red-shifted, in contrast to what is normally seen for the scattered $30 \mathrm{~nm} \mathrm{Au} \mathrm{NPs,}$ with their surface plasm absorption. This red shift is consistent with Mie's theory and the development of an isotropically functionalized aggregate in comparison to larger polymer structures $[339,340]$.

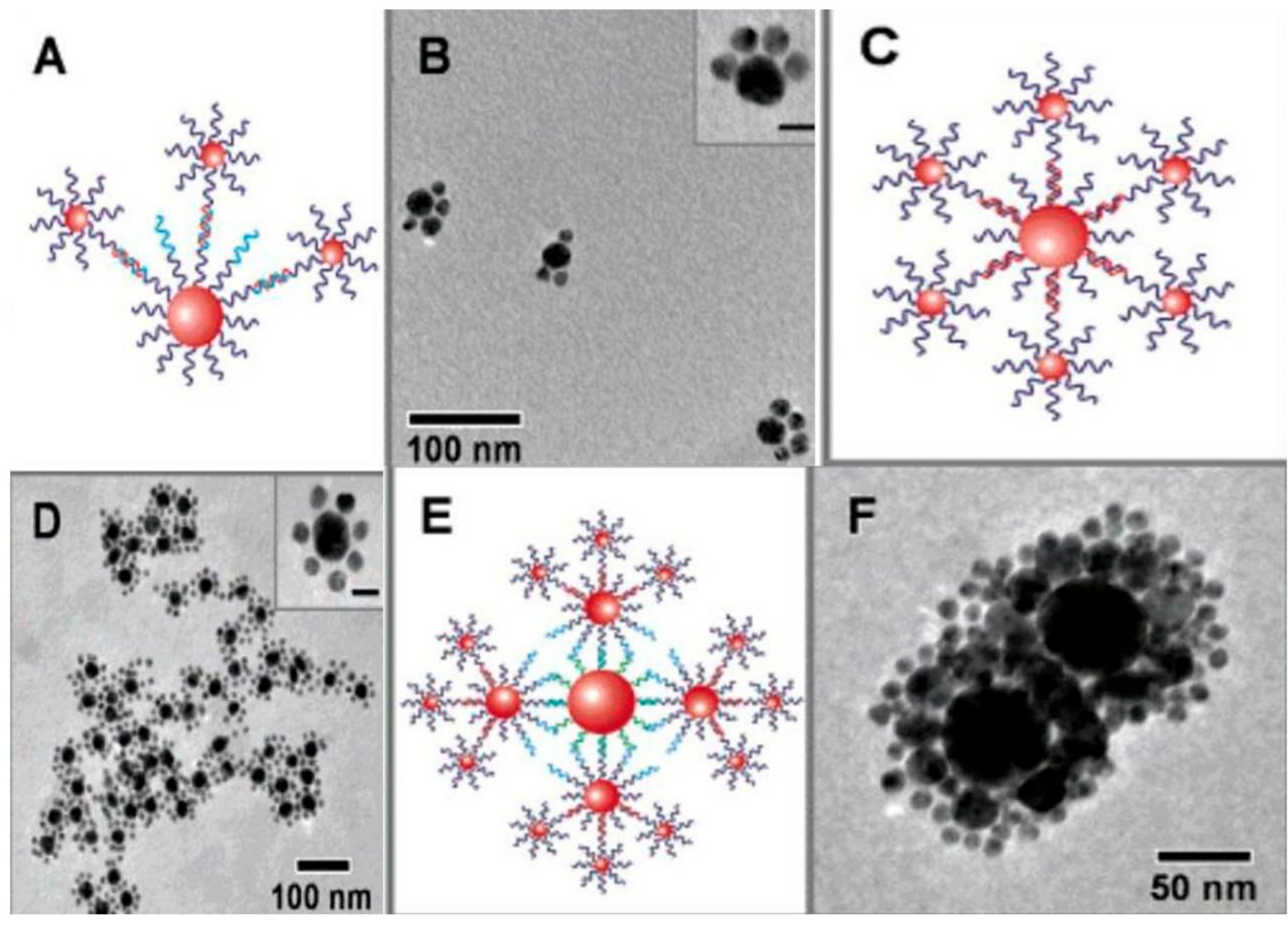

Figure 49. Directional assembly of the dendrimer-like structures of asymmetrically functional Au NPs (A,B) cat paw (C-F). Installation: bar scale) $20 \mathrm{~nm}$. Copyright 2017 RSC, re-printed with reference permission [340].

Satellite structures with an average diameter of $152(10 \mathrm{~nm})$, which is about the diameter of the satellite structure from two different sizes of Au NPs blocks and DNA interconnections are also allowed by dynamic calculation. The asymmetrically functional particles are likely to trigger a denderimer. They have, for example, ynthesized 30 and $60 \mathrm{~nm}$ Au NP satellite structures identical to those in Figure 49C,D. The dendrimer-like structures are hybridized by more oligonucleotides that have not been expanded. A third kind of nanostructure that looks like $30 \mathrm{~nm}$ particles in an asymmetrical $13 \mathrm{Nm}$ Au NP with opposite oligonucleotides (see Figure 49E,F). This three-component structure shows that it can be used with this method and asymmetrical particles to manage the assembly by at least three separate Au NPs in systematically in-expected heterostructures. Asymmetric $\mathrm{Au}$ NPs functionality was demonstrated with oligonucleotides and it was known that DNA operations allow MMPs both to alter DNA modified Au NPs locally and to help detach 
and distill anisotropically functionalized Au NPs. The Au-functionalized NP's in this way display highly directed discrimination with additional nanoparticles for hybridization, enabling the preparation and montage of distinguishing nanoparticles such as satellite, cat paw and dendrimer-like structures. This is a significant aspect towards the construction of valence into nanoparticles, which allow refined nanostructured materials to be carefully planed and preparationed.

\section{Surface Chemistry of Au NPs for Various Applications}

\subsection{Au NP for Health Applications}

7.1.1. Surface Chemistry of Au NPs Enabled Prophylaxis

Au NPs do not induce undesirable immune responses [341-343]; they act as concepts in the packaging of vaccines, to increase the thermal stability of vaccines from hours to months at room temperature (see Figure 50) [344].

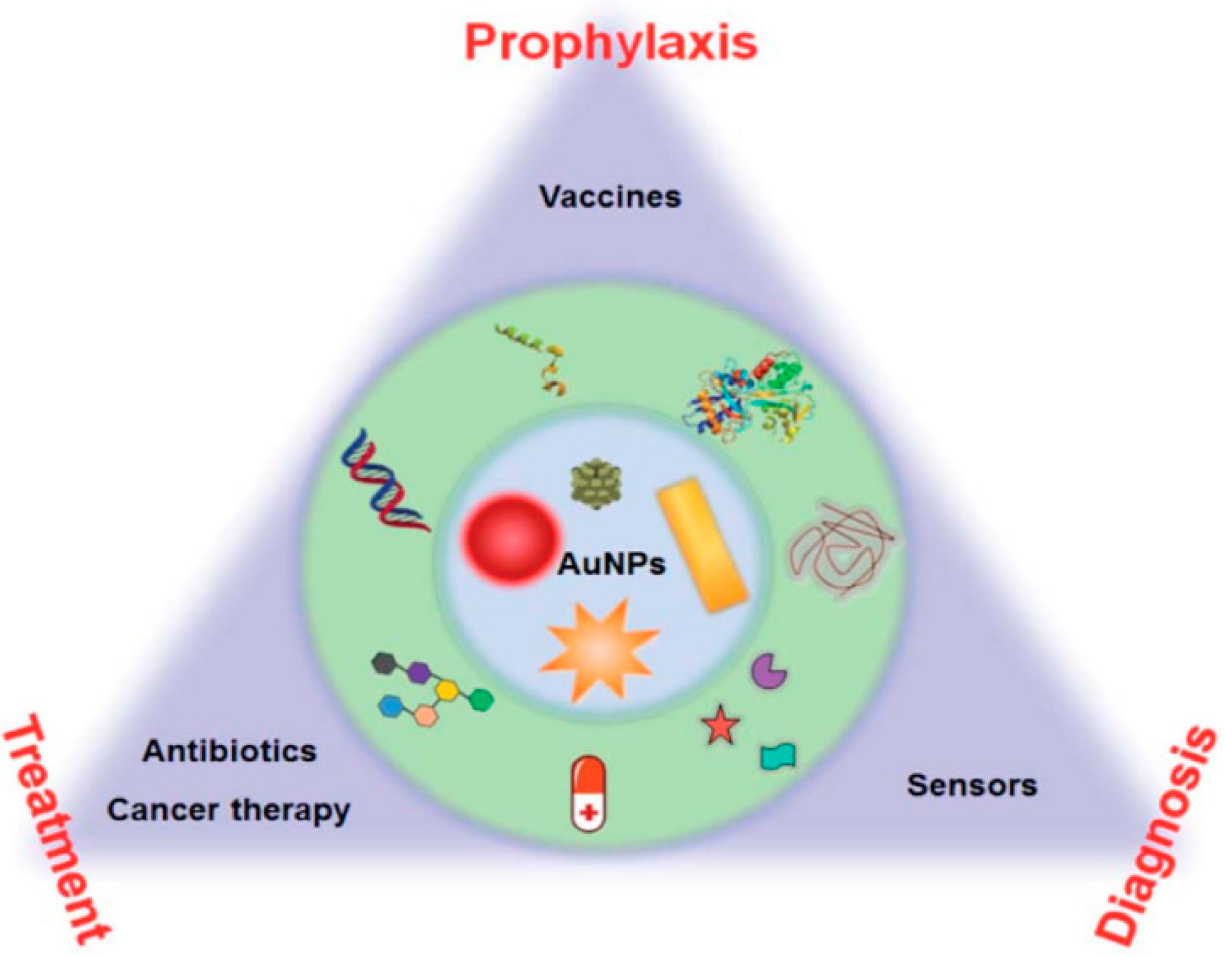

Figure 50. Schematic examples of implementations of prophylaxis-based health-related healthcare applications. Copyright 2016, Nat, reprinted with reference permission [345].

\subsubsection{Au NPs Modified Proteins/Peptides as Vaccines}

Through physicochemically, the Au NPs conjugate with the protein-based antigens, which are widely employed for vaccines, this makes the vaccines fruitless by delaying the antigen place. The Au NPs surface distracts their conjugation with proteins. Several polymers are added in order to enhance Au NPs to preserve and protect the effectiveness of vaccines for the bioactive immobilisation of protein antigen. Poly(4)styreneesulfonic acid (PSS)/PSSco- maleic acid (PSS-MA) is employed in the alteration of Au NPs [346,347]. The Au NPs with PSS-MA can, therefore, simply conjugate virus-envelope proteins which display the epitope to produce exact antibodies [348]. Mainly, PSS-MA can alter Au NP chemical surface properties and greatly decrease cytotoxicity of cetyltrimethyl ammonium bromide (CTAB). A $40 \mathrm{~nm}$ spherical complex of Au NPs proteins produced the maximum degree of antibodies defined in comparison to the other particles. Improved cytokine responses can be increased by factors such as Au-size NPs shape. The orientation of proteins on $\mathrm{Au} \mathrm{NPs}$ that characterize their bioactivity in the electro-static binding interference conjugation. Important experiments were carried out with the application of different processes: 
calorimetry of the isothermic titration, quenching of fuorescence, dynamic light dispersion, liquid chromatography mass spectrometry and spectroscopy of the circular dichroism, X-ray photoelectron spectroscopy and enzymatic activity tests [349-351]. Electrostatic binding also greatly decreases the bioactivity of proteine denaturation. Additional scheme used for antigen Au NPs vaccines has been applied to the tetraethylene glycol spacer between Au NPs and proteins to secure proteins and forbidden denaturation [352] of protein antigens. The examiner has described the method of immobilizing malaria surface proteins with aldolization using glutaraldehyde [353] on Au NPs. The close chemical relation strengthens the complex's stability and can escape the removal of protein antigens in vivo. The result of glutaraldehyde will lead to a high crosslinking of proteins, amidation mediated by chemistry and clicking chemistry of EDC/NHS (coppercatalyzed azide-alkyne cycloaddition). The use of unique peptides as an antigen subunit for Au NPNPs, instead of a whole protein, is another energetic technique for emerging vaccines [346,354-356]. The high attraction between sulfydryl and Au will approve a maximum relation through the morally added use of amore cysteine to a peptide, [357]. In order to adjust Au NPs as a virus vaccine, scientists have used the synthetic foot and mouth virus of cysteine $[358,359]$. In order to adjust $\mathrm{Au}$ NPs as a virus vaccine, scientists have used the synthetic foot and mouth virus of cysteine [342]. The antibody response compared with conservative protein vector Peptide-Au NPs has pressed for three-fold increase. Similarly, the Au NPs modified by cysteine-terminated Matrix-2 protein are amended as the vaccine against an influenza virus [360]. Intra-nasal vaccination experiments demonstrated that the vaccine M2e-Au NP (cytosine-guanine-rich adjuvant $\mathrm{CpG}$ oligonucleotide) would fully accommodate mouse neartest with the infant $A$ virus. Meanwhile, their surface and antigenic act may strongly be changed by the distance between a certain peptide and Au surface. The efficacy of the vaccine can be improved by a long chain thiol or short SH-PEG spacer to increase the dense collection and surface elasticity of peptides on Au NPs [361].

\subsubsection{Au NPs Modified Carbohydrates as Vaccines}

Glycoproteins wrapped in specific glycan chains are piles of protein antigens. These glycans are capable of inducing immunogenic reactions and contribute to the detection of glycanic antibodies [362]. In envelope protein gp120, strongly mannosed glucose was detected by the only 2G12, monoclonal carbohydic antibody, mainly neutralizing Type1 human immuno sufficiency virus (HIV-1). The target antigen for improving the HIV vaccine may be high mannose glycans rather than the entire gp120, with 2G12-like antibodies [363] made. These carbohydrates, however, display poor appeal and are not able to allow Au NPs to achieve their proper output. Researchers improved oligomannosides with a long amphiphilic linker containing thiol [364]. The linker helps the Au NPs forming an Au-S bond bind carbohydrates. The aliphatic fragment of the linker makes self-assembled monolayers with a dense package, but elasticity, availability of unspecific protein adsorption is given by external multifaceted entities and carbohydrate antigenes are approved for immunogenous efficacy. The glycan Au NP products are highly successful in binding neutralization of 2G12 and block 2G12. In contradiction with HIV, Glycan Au NPs will possibly be a beneficial, flexible and multifunctional vaccine. Curred dedication for cancer immunotherapy has now been exhausted in the synthetic cancer vaccines. Breast cancer cells above carbohydrates connected with direct mucin, for example GalNAc (Tn-antigen glycan). Tn-antigen glycan, combined with Au NPs, may produce significant anti-cell titers. In accordance with an in situ reduction method for producing Au NPs, the Tn-antigen-plotted polymer has been applied by researchers to polymers [365] (see Figure 51). Complete titers applaud the strong possession by Au NPs with carbohydrate density for the incidental communications between B-cell stimulation immune reaction and antibody development. 

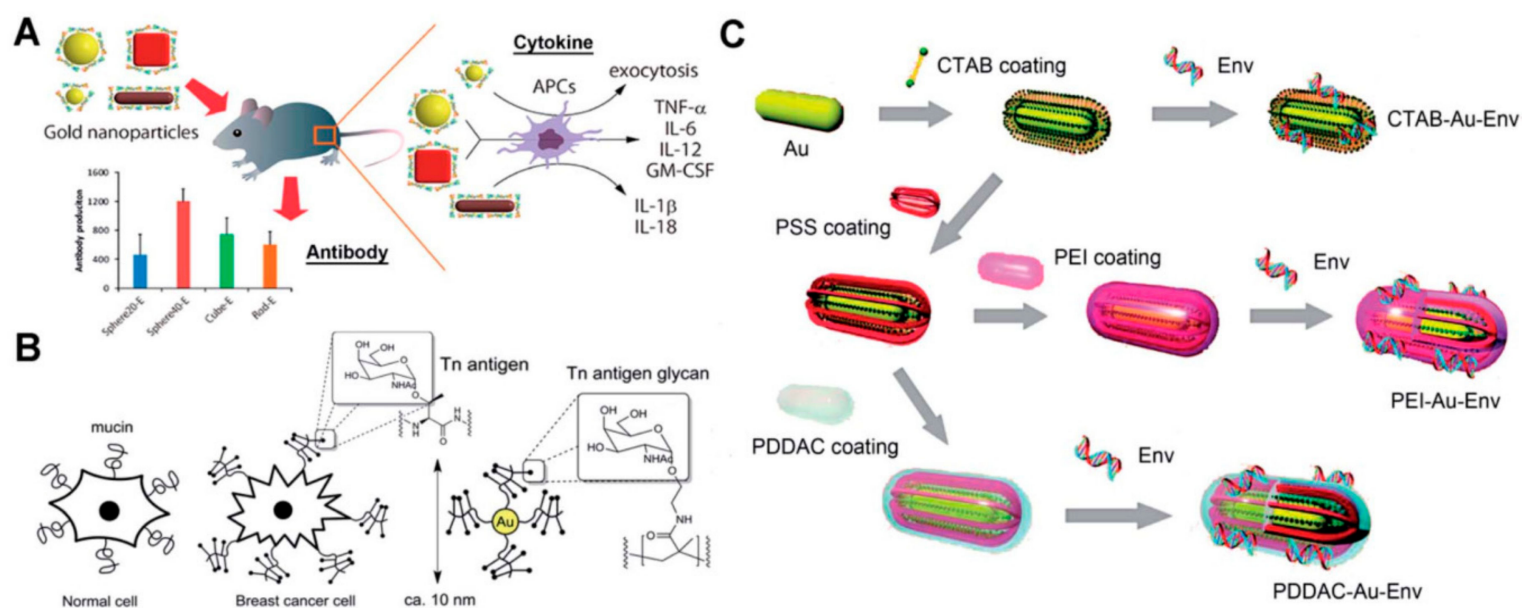

Figure 51. Schematic representation as synthetic vaccines of the different antigen conjugated Au NPs. (A) an antigen-based West Nile virus protein (WNVE) attached to PSS-MA coated Au NP (different sizes and forms) through electrostatic activity directed to virus vaccinations. Copyright 2013 ACS, reproduced with reference permission [348], (B) Tn antigen glycopolymer antigen, covalently modified in Au NPs for cancer vaccines, associated mucinal cells. With reference authorization [366], 2013 ACS Copyright. (C) HIV-1 Env plasmid DNA as an antigen attached to surface rods such as Au NPs for virus vaccines (coated with different polymers). Copyright 2012, ACS, reproduced with reference permission [367], Copyright 2012, ACS.

\subsubsection{Au NPs Modified DNA as Vaccines}

In addition to humoral immune reactions to other vaccines, DNA vaccines can give a longer-term cell immunity [366]. Their development and storage are harmless and low and therefore simultaneous immunization may be done in opposition to many antigens or pathogens. DNA-modified Au NPs like nanocytes can significantly increase antigen cell approval (APCs) and prevent nuclease DNA degradation [367]. A single stranded DNA (SSDNA) is able to bind to Au NPs by means of nucleobase chemisorption. However, double-stranded DNA (dsDNA) is not binding due to the base pairing and mounding of for example a plasmid [294]. The chemical surface changes of the Au NPs are necessary if plasmid DNA is combined with the vaccine. For Au NPNPs' surface coverage, researchers have synthesized low molar mass chitosans (6 kDa, Chito6) [368]. The Chito6-Au NPs was shown to increase HBV-plasmid DNA conjugation. Compared with the bare intensity of the DNA vaccine, intramuscular addition results of the mice showed stronger humor (10 times) and cellular ansughts. In order to detect the effect of Au NP surface chemistry on vaccination, studants have formed rods such as Au NPs (pSS coated, polyethylene/PEI coated, CTAB coated and poly(-diallydimethyl ammonium chloride)/PDDAC) [367]. These formed Au NPs can combine HIV-1 plasmid DNA through physical and static adsorption. Increasing cell approval (APC) and stimulating dendritic-cell maduration (DC) in order to retract and improve immunological reactions, may benefit the immune-genicity of DNA $\mathrm{Au}$ NPs. The vaccine power of DNA-Au NPs has changed significantly due to different surface chemistries. There still is much space to explore the best conjugation of dsDNA and tough immunogenic action in surface chemistry of Au NPs.

\subsubsection{The Au NPs Surface Enables Diagnosis}

Au NPs functionality for metal ions, proteins, nucleic acid, bacteria and cells have been identified diagnosed [196,216,369-372].

\section{Metal Ions Diagnosis}

Metal ions combine chemically and physically into many categories, including thiol, hydroxy, amine and carboxyle. These can bind or remove the surface-functioning ligands after the injection of a sample and influence the assembly of Au NP [294] through modification of DNA oligonucleotides onto Au NPs. The exact reaction between analyte 
ions and chelating ligands is used to collect and do non-accumulate au-NPs for improved colorimetric examinations [165]. The other surface varying steps of the Au NPs can also be used to enhance metal ions such as $\mathrm{Na}^{+}, \mathrm{K}^{+}, \mathrm{Pb}^{2+}$ and $\mathrm{Cd}^{2+}[174,373]$. Au NPs formed with ammonium-group quaternary thiols can perceive mercury (II) sensitively and selectively [224]. $\mathrm{In}_{\mathrm{Hg}}{ }^{2+}$, the altered ligands may be withdrawn, thereby affecting the Au NP aggregation. Thiolates on Au NPs can be photooxidated in sulfonates that speed up the aggregation process under solar light irradiation. In some studies, $\mathrm{Hg}^{2+}$ can displace rhodamine B isothiocyanate from Au NPs, ensuring a fluorescence re-clamation, when using modified Au NPs with poly(ethylene glycol) and rhodamine B [225], $\mathrm{Hg}^{2+}$ can displace rhodamine B isothiocyanate from Au NPs, thus ensuring fluorescence reclamation. The highly sensitive metal ion can be classified as a catalyst by the metal ion that catalyzes various processes. Colorimetric $\mathrm{Cu}^{2+}$ detection is the other application for the alkynes and the azides are catalytized with the ion of copper $[\mathrm{Cu}(\mathrm{I})]$ to create a solid chemical bond following a covalent aggregation of Au NPs (see Figure 52A) [213]. These $\mathrm{Cu}(\mathrm{I})$-catalyzed 1,3-dipolar cycloading (CuAAC) techniques have the following advantages:a highly stable and selective chemical covalent mixture of azides and alkynes [374]. These $\mathrm{Cu}(\mathrm{I})$-catalyzed 1,3-dipolar cycloading (CuAAC) techniques have the following advantages: a highly stable and selective chemical covalent mixture of azides and alkynes. The $\mathrm{Cu}(\mathrm{I})$ catalyst promotes these processes that affirm his high degree of sympathy. In order to distress the catalytic activities of the Au NPs, metal ions may be reduced and deposited on Au NPs forming bimetalic nanoalloys. Such a device can recognize several metal ions, including $\mathrm{Hg}^{2}, \mathrm{~Pb}^{2}$ and $\mathrm{Cr}^{3+}$ ions [375,376] in a selective and sensitive way. The catalytic activities intermediated by metal ion is routinely inspected and multiplex processes imaginable have been completed [372,377].

The improved surfaces of the Au NPs are important for the colorimetrical find of metal ions in certain functional groups. The persuaded aggregation/disaggregation of $\mathrm{Au}$ NPs with metal ions depends on the interface of the improved molecules between metals and the surface. These can be accomplished by changing the surface improved molecules and removing and linking them, thereby altering their status. In the meantime, catalytic processes dependent on metal ions can also be used to boost these sensors. These sensor forms can be highly discriminatory. Other forms of catalytic processes dependent on metal ions are considered overly possible during these investigations. The progress of $\mathrm{Au}$ NP-based sensors for the identification of metal ions can be catalyzed by innovative types of chemistry linking metal ions.

\section{The Surface Functionalized Proteins-Au NPs with Probes}

Proteins, DNA and RNA can selectively be detected by surface functionalized proteins$\mathrm{Au}$ NPs with probes such as antibodies and nucleic acid aptamers. This subject relates primarily to the identification of proteins through the surface of Au NPs. The maximum number of these processes depends on the growth or aggregation of Au NPs mediated by the antibody functionalized Au NPs and the application enzym: protein-protein interaction [378], protein-aptamer interaction [379] and protein-carbohydrate interaction [380] are commonly used to modify and detect proteins easily and sensibly. Such approaches are simple and successful but lack universality. Au NPs with alkynes and azides were functionalized to integrate Au NPs into the old but potent enzyme-linked immuno-sorbent examination (ELISA). CuAAC exists on the surface of Au NPs, resulting in Au NPs being aggregated (see Figure 52B) [215]. They are commonly used to modify and detect proteins easily and sensibly. Such approaches are simple and successful but lack universality. Au NPs with alkynes and azides were functionalized to integrate Au NPs into the old but potent enzyme-linked immuno-sorbent examination (ELISA). CuAAC exists on the surface of Au NPs, resulting in Au NPs being aggregated (see Figure 52B) [381]. Compared to the predictable enzyme related immuno-sorbent assay, these colorimetric immuno-assays show greater sympathy and power (ELISA). Numerous enzyme mimic activities are exhibited by $\mathrm{Au}$ NPs that can substitute natural enzymes for bio-marker recognition. Surface chemistry 
may prevent or increase the enzyme-mimicking behaviour of Au NPs. No peroxidase action is performed by CTAB modified Au NPs prepared via the seed growth process. Silver ions $\left(\mathrm{Ag}^{+}\right)$have a strong surface attraction of $\mathrm{Au} \mathrm{NPs}$, thereby disrupting the CTAB membrane and enhancing the activity of peroxidase (Ag+ gate peroxidase activity of $\mathrm{Au}$ NPs (Figure 52C)) [382]. Acetylcholine sterase (AChE) can hydrolyze acetyl-thiocholine to thiocholine as a critical neurological disease biomarker. The thiocholine generated can organise $\mathrm{Ag}^{+}$and prevent the action of $\mathrm{Ag}^{+}$gate peroxidase by Au NPs. For fluorescence detection of acetyl-choline in blood models, there was a related research study using bimetallic $\mathrm{Au} @ \mathrm{Ag}^{0}$ core-shell nanoparticle-referred peroxidase activity [383].

A

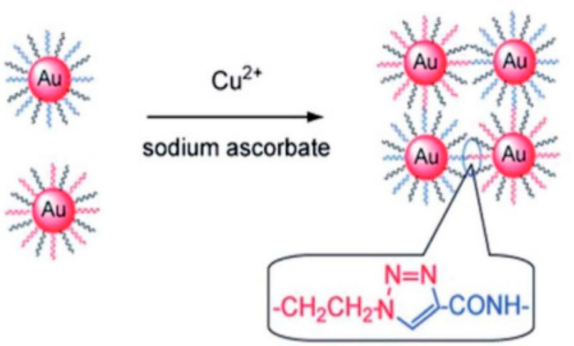

C

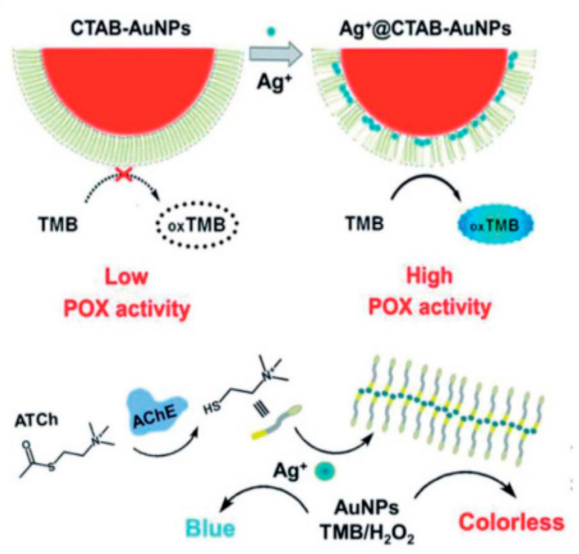

E

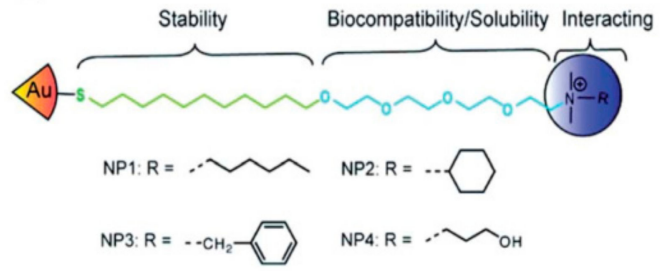

B

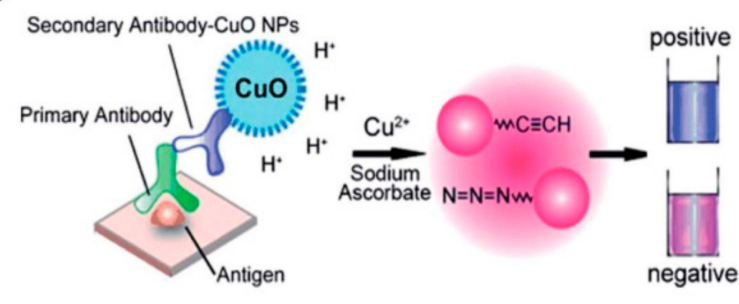

D
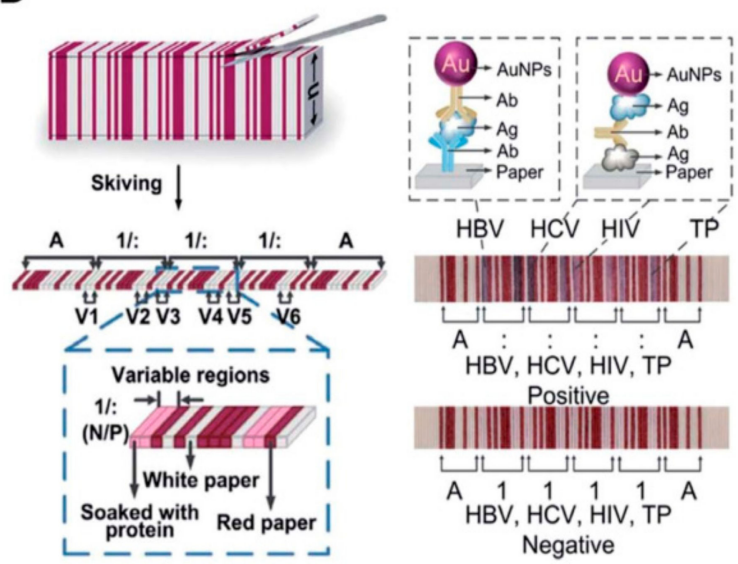

$\begin{array}{llllll}10^{7} \quad 10^{6} & 10^{5} & 10^{4} & 10^{3} & 10^{2} & \text { (bacteria/mL) }\end{array}$

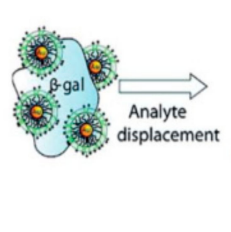

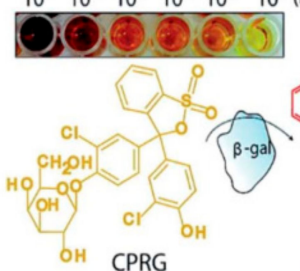

OH CPRG

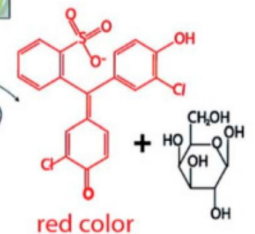

Figure 52. Au NPs allow sensing and diagnostic surface chemistry. (A) The 1,3-diogravic azide and alkyne (CuAAC) catalyzed $\mathrm{Cu}(\mathrm{I})$ occurs at the Au NP surface, which is the product of Au NPs being aggregated. Copyright 2008 Wiley-VCH, reproduced with reference permission [213] (B) CuAAC-based colorimetric immunoassay occurs on an Au NP surface. Copyright 2011 Wiley-VCH, reproduced with reference permission [215] (C) Silver ions ( $\left.\mathrm{Ag}^{+}\right)$interrupt the CTAB membrane and regenerate $\mathrm{Au} \mathrm{NP}$ peroxidase activity ( $\mathrm{Ag}^{+}$gate peroxidase). Reproduced by reference authorisation [382] Wiley-VCH copyright 2018. (D) Set sheets of paper for multiplexed immuno-assays shall be shirked in paper barcode chips (PBCs). Copyright 2017 American Association for the Advancement of Sciences, reproduced with reference permission [384] (E) Au NPs are electrostatically associated with b-gal ammonium quaternary ligands. Bacteria analyte transfer b-gal and repairs activity of the enzymes. If a naked-eye reading is used, the evacuated b-Gal can turn a pale-yellow chromogenous substrate into a red product. Copyright 2011, ACS, reproduced with reference permission [371], Copyright 2011, ACS. 
Based on its distinctive visual characteristics, the most fruitful applications of Au NPs are sensing uses. The high elimination coefficients of Au NPs are used by these types of colorimetric sensors and can give naked-eye readouts with a low detection limit. The act of recognition is interrupted by surface features of Au NPs for most of these applications. LFAs can transform from qualitative inspection to quantitative recognition with improved sensitivity by functionalizing Au NPs with signal assessments such as enzymes and fluorescent molecules [385]. For appropriate processes, components can be lyophilized on the LFA strip [386]. A paper-based barcode assay system based on barcode scanning will provide a more objective and reliable reading separately from the illustrative, fluorescent and chemi-luminescent reading of LFAs [384]. For multiplexed immuno-assays, the fixed sheets of paper are shirked into paper based barcode chips (PBCs) (see Figure 52D) [387]. This explores the platform that enables various biomarkers to be discovered and provides multiplexed implications within several minutes. For point-of-care finding of proteins in resource restricted sets, colorimetric sensors are suitable. The optical and surface characteristics of Au NPs provide a perfect stage for these sensors to advance. Although Au NP-based assays include a moral recognition act, the following fragments will advance these types of examinations: full automation, improved presentation of results and prolonged stability. Surface chemistry gives Au NPs the opportunity to identify targets while also increasing old sensor analytical recitals (such as sensitivity and selectivity). When combined with other innovations, such as microfluidics, Au NPs-based inspections may have provided an automated operation recital. In this area, we believe there is still much progress to be made, particularly in the surface chemistry of Au NPs.

\section{Recognition of Bacteria, Viruses and Cells via Au NPs}

$\mathrm{Au}$ NPs have been widely used for recognizing bacteria and cells [388]. The chemistry of the Au surface gives the opportunity to locate bacteria or cells. Extreme processes depend on antibodies and on Au NP evaluations built to detain bacteria and cells. For these applications, Au NPs enhanced with modified small molecules may also be used. The interaction between the viral hemagglutinin (HA) protein and the host glycan receptors can be tested by glycan functionalized Au NPs (Au NPs). To identifyavian infuenza visually, this technique is used [389]. Au NPs may be reserved for trimeric HAs or viruses, resulting in gAu NP aggregation. This naked-eye readout takes care of the quick and in-depth detection of harmful viruses. Quaternary ammonium ligands are functionalized with Au NPs, which can electrostatically stabilise b-galactosidase (b-Gal, see Figure 52E) [371]. Quaternary ammonium ligands can simultaneously stabilise Au NPs and increase their solubility and biocompatibility. The functionalized Au NPs of the quaternary ammonium ligand will resist the action of $b-G a l$. When the anionic-surface analyte bacteria were applied to the Au NPs, b-Gal transferred to the Au NPs surface and mended the enzyme effort. For the naked-eye readouts, the moved $b$-Gal will alter a pale yellow chromogenic substrate into a red substance. Compared to the $\mathrm{Au}-\mathrm{S}$ bond, physical adsorption between amino groups and $\mathrm{Au}$ is weak; however, the likelihood of colorimetric bacterial finding is given by this method. In a one-pot reaction, modified Au NPs are prepared from D-AlanylDalanine (DADA) [390]. In extremely acid or alkaline conditions, the resulting Au NPs remain stable. DADA will recognise peptidoglycan on the bacterial surface in the bacteria society, influencing the aggregation of Au NPs due to the lack of DADA shield. This colorimetric assay will distinguish between Staphylococcus aureus and Staphylococcus aureus immune to methicillin from ascite models in patients. In fact, colorimetric sensors based on physical adsorption and chemical crosslinking are largely detected based on $\mathrm{Au}$ NPs. For colorimetric sensors, molecular forces and chemical processes between target analytes and Au NPs are vital. We can precisely control and scheme the surface characteristics of Au NPs through surface chemistry and increase their utility. Other biochemical processes can also be used for surface modification of Au NPs, separately from antibodies and molecules we have designed beyond. The integration of finding and behaviour into one combined platform; meanwhile, this demonstrates disproportionate 
value in the provision of bacterial sicknesses. The possibility of these uses is discussed by the surface chemistry of Au NPs.

\subsubsection{The Surface Chemistry of Au NPs Enables Treatment}

Functionalized Au NPs as Antibiotics

Although bare Au NPs have no antibacterial properties, surface functionalized $\mathrm{Au}$ NPs are used as antibiotics with enhanced antibacterial movement compared to current antibiotics or medicines with medications, vaccines and antibiotics [391,392]. Nanoparticles can deliver antibiotics or drugs to bacteria competently and increase the concentration of drugs in targeted bacteria, thus refining the antibacterial properties of old antibiotics. Maximal experiments on antibiotic-dependent nanoparticles are based on antibiotic or drug functionalization. Recent research shows that Au's surface chemistry offers strong antibacterial features for Au NPs. Surface modification by the Au-S bond of certain nonantibacterial molecules on Au NPs will convey antibacterial features to these Au NPs. In 2010, amino-replaced pyrimidine, such as 4,6-diamino-2-pyrimidinethiol (DAPT), functionalized Au NPs (Au-DAPT) have been investigated as antibacterial agents targeting multi-drug resistant Gram-negative bacteria, but none of the components have antibacterial potential on their own. The positively charged DAPT groups will increase the permeability of Au NPs to the bacteria's outer membrane, thereby refining the beneficial efficacy. By modifying the membrane strength and avoiding the subunit of ribosomes, the Au-DAPT exerts its antibacterial activity [393]. Numerous other accounts also record strong antibacterial activity of cationic and hydrophobic functionalized Au NPs [394-396]. The synergistic properties of non-antibiotic drugs and DAPT on Au NPs in conflict with superbugs are additional stimulating findings [397]. The Au-improved NPs' antibacterial activity is associated with both GP-positive and Gram-negative bacteria, consisting of superbug-driven medication. This is after co-functionality with non-antibiotic drugs and DAPT.

$\mathrm{Co}$-functioning of $\mathrm{Au}$ NPs with antimicrobials and other functional components is a common procedure that offers more dominant biomedical antibiotics based on $\mathrm{Au}$ NPs [398]. Further reading reveals that N-heterocyclic molecules also have the same effect on [399]. The Au NPs show broad spectrum antibacterial actions in contradiction to even superbugs that repel most antibiotics by surface functionalization with an $\mathrm{N}$-heterocyclic molecule. Physical adsorption is also used as a surface modification technique for Au NPs separately from using the $\mathrm{Au}-\mathrm{S}$ bond. Via physical adsorption between amino groups and $\mathrm{Au}$ NPs, pharmaceutical intermediates such as 7-amino-cephalosporanic acid (7-ACA), 6-amino-penicillanic acid (6-APA) and 7-amino-desacetoxycephalosporanic acid (7-ADCA) will adsorb Au NPs on the surface [400]. Via cell membrane interruption and lysis of bacterial cells, these pharmaceutical intermediate functionalized Au NPs will defend against Gram-negative bacteria consisting of both laboratory antibiotic-sensitive strains and clinical multidrug-resistant separates. Numerous amino-saccharides have similar structures to the bacterial peptide-glycan. The functionalized Au NPs (Au-GluN) of Dglucosamine $(\mathrm{GluN})$ will disrupt bacterial peptidoglycan, leading to the loss of bacteria [401]. Further research shows that we can find a small range of antibiotics by functionalizing Au NPs with amino-saccharides [402]. The Au-GluN will move through the cell wall and change the cell membrane's structure to convince the cell to lose bacteria.

Some Au NP antibacterial coverings and coatings are advanced to thoroughly discover the use of Au NPs-based antibiotics. Helical healing issues arise in bacterial poisons. Strong bacterial poisons can also contribute to sepsis approximately. Electrospinning allows nano-sized polymer fibers to be synthesized according to human requirements. In Poly(3-caprolactone)/gelatin solution under electrospinning, we doped intermediate pharmaceutical Au NPs to [401]. Relative to physical adsorption techniques, the electrospun nanofibrous scaffolds provide a homogeneous coating of nanomaterials on fibres. With the humiliation of poly(3-caprolactone)/gelatin nanofibrous scaffolds, anti-bacterial Au NPs can be consistently free to provide constant antimicrobial capacity. This mechanism can also be recognised by co-electrospinning Au NPs and poly-poly-capped indole derivative 
co-electrospinning (-lactic-co-glycolicacid) [403]. Indole does not show any antibacterial activity as a major derivative in the food and drug industries. The resulting AI-IDs demonstrate strong antibacterial activity in contradiction to multidrug resistant superbugsafter physical adsorption of indole derivatives onto Au NPs (AI-IDs). Bacterial cellulose (BC), a popular biomaterial treatment for tissue engineering, shows advantages of high biocompatibility: the capacity to absorb water and mechanical strength. When a BC membrane is saturated in an Au-DAPT solution, through physical adsorption, Au-DAPT will adsorb on the BC membrane, which has implications for wound healing in an antibacterial dressing (BC-Au-DAPT nanocomposites) [404]. The BC-Au-DAPT freeze-dried nanocomposites are highly stable and, after one year of storage, maintain their antibacterial action. Another use of antibiotics based on Au NPs is their use as antibacterial coatings for medical strategies. The surface of the medical devices is negatively emotional later in the plasma action. Positively charged surface Au-DAPT will adsorb stably on the surface of medical devices by electrostatic self-assembly [405]. Thus, the immobilised Au-DAPT will destroy antimicrobial constituents if nosocomial medical device contamination is a secure but proficient antibacterial method. Au NPs smaller than $2 \mathrm{~nm}$, often referred to as Au nanoclusters (Au-NCs), have confirmed antibacterial activity when quaternary ammonium (QA) salts are functionalized [406]. For bonding Au-NCs, one end of the QA has a sulfydryl group and the other end has positively charged ligands that can increase membrane permeability and decrease bacteria drug efflux. In comparison to multidrug-resistant Gram-positive bacteria, these types of antibiotics can battle in vivo and have no detectable cells. The time of circulation is equivalent to that of vancomycin (a widely used antibiotic), which confirms its efficacy as an antibacterial agent. Additional work using antimicrobial peptide selfassembly on Au nanodots demonstrates superior antimicrobial activity by decomposing the bacterial membrane [407]. Meanwhile, on the basis of Au-S bond-based conjugation of Au NPs and the unique thiol molecules, the outstanding antibacterial capacity is built. Millimola quantities of glutathione can competitively bind to Au NP surfaces via the $\mathrm{Au}-\mathrm{S}$ bond in the blood stream and cells. The disinterest caused by the movement of the particular thiol molecules will significantly reduce the Au NPs complex's antibacterial capability. The stronger chemical bond to conjugate Au NPs and particular molecules is likely to avoid invalidation caused by glutathione movement. By developing an Au-Se bond instead of an Au-S bond, researchers used selenol modified peptides with dye to synthesise fuorescent Au NPs [408]. The more stable Au-Se bonds are able to speechless thiol compound intervention. The carbene-Au bond can also be employed for bridging functional N-heterocyclic carbene molecules and Au NPs [409]. In comparison to thermal injury, the N-heterocyclic carbene-improved Au NPs / Au-NCs display high fluorescence quantum output and high stability [410-412]. Based on the same value, both the Au-Se bond and the stunned thiol interference should be capable of the more stable carbene-Au bond. Au's surface chemistry confers strong antibacterial properties on Au NPs. These forms of antibiotics can kill superbugs and reduce the resistance of bacteria to drugs. We believe that surface functionalization of $\mathrm{Au}$ NPs with other types of molecules, apart from pyrimidine, such as N-heterocyclic molecules, aminosaccharides, indoles, pharmaceutical intermediates and QA salts, will allow the improvement of new antimicrobial tools. In the meantime, more studies on the properties of dissimilar sizes, shapes and surface goods of Au NPs on antimicrobial properties are still needed.

\section{Functionalized Au NPs for Cancer Therapy}

One of the greatest deadly human illnesses is cancer. Because of the range of such a chronic illness, distinction of personal appearance and lack of effective miracle drugs, cancer therapy is still a persistent scientific test. The most real mode of cancer action is currently surgery in combination with radiotherapy and chemotherapy. However, it is vital to advance new treatment plans and extremely specific medicines for the simply invasive and metastatic products of cancer cells and the increasing drug confrontation. Nanomedicine tackles unique mechanisms and has emerged as an encouraging plan to 
improve old therapeutic efficacy. It may advance the differential improvement for imageguided therapy and cancer-specific delivery of chemotherapeutic agents for joint therapy by promoting the target selection of cancer. Among various nanomaterials studied for cancer therapeutic uses, Au NPs were mostly studied taking advantage of their distinctive chemical, electrical and optical characteristics and outstanding biocompatible characteristics, as well as the ease of synthetic activity and precise rheostat over their physicochemical characteristics [7,413,414]. The high attractiveness of Au NPs to bind thiols, amines and polymers offers an effective way of presenting reactive functional groups that can be used for targeting and conjugating therapeutic agents (e.g., antibodies, peptides, aptamers and carbohydrates, drugs, radionuclides, photosensitizers, siRNA and genes). The high X-ray and near-infrared (NIR) light absorption coefficient will openly award Au NPs for cancer therapy with the effect of radio-sensitization and photothermal control. Multifunctional nanomedicine can achieve distinctive characteristics that can not be accomplished with $\mathrm{Au}$ NPs alone by combining Au NPs into other nanoplatforms, such as liposomes. In the development of useful nanomedicines that are specialised in multi-modal therapeutic uses in cancer care, Au NPs are talented. In vivo, Au NPs demonstrate the effect of radiation dose improvement and have created great interest in the field of radio-sensitization based on Au NPs for oncology. The survival of mice with subcutaneous EMT-6 mammary carcinoma can be substantially improved by X-ray irradiation and activity with Au NPs [415]. In addition, researchers have discovered novel methods of radio-sensitization for chemical improvement (DNA damage and radical production) and biological improvement in physical improvement (ROS-induced oxidative stress, inhibition of DNA healing and cell cycle disruption) [416]. The effectiveness of cellular approval can greatly enhance the therapeutic effect. For Au NP dependent nanomedicines, the size and surface charges are significantly affected to monitor the efficacy of their cellular uptake [417]. The selective accumulation at tumour sites was aided by circulating Au NPs with enhanced porous design and retention (EPR) effect assistance. The cellular absorption of highly negatively charged surface Au NPs is very difficult. To increase cellular uptake, neutral or positively charged surfaces are helpful [418,419]. Researchers have researched the size-dependent radio-sensitization of Au NPs using an U14 tumour bearing mouse model by surface functionalization with PEG-SH coatings [420]. Compared to smaller or larger PEG-Au NPs, PEG-Au NPs (10-30 nm) demonstrated higher radio-sensitivity for radiotherapy. By modifying the surface chemistry of Au NPs with coating molecules, with improved Au NP build-up in tumours, researchers may produce separate mutual radio-sensitization. Metabolizable ultra-small Au NPs with a biocompatible covering ligand (glutathione, GSH) (core size $1.5 \mathrm{~nm}$ ) strongly enhance cancer radio-therapy [421]. Through the enhanced EPR effect, the GSH-Au NPs can be collected in growths differently. GSH-Au NPs can be professionally cleared by the kidneys following treatment, which eliminates any potential side effects. Photo-thermal therapy uses NIR light/laser as the energy source to produce high temperatures to destroy cancer cells instead of using harmful high-energy rays and pollutants (radiotherapy). Plasmonic Au nanoshells have been identified in previous studies to demonstrate photo-thermal properties and are thus used for near-infrared thermal treatment of tumours [422,423]. Rod-like Au NPs with a tunable LSPR band associated with the nanoshells compared to the nanoshells, rod-like Au NPs with a tunable LSPR band between 650 and $950 \mathrm{~nm}$ (falling in the NIR region) are considered to have great potential for beneficial nanomedicine [424-426]. In addition, multi-functional nanomedicines can achieve a combined scheme to advance specific cancer treatment by combining the photo-thermal effect with pre-loading anti-cancer drugs or photosensitizers. The surface chemistry of Au NPs at this stage reveals a crucial role in regulating the loading efficacy of these therapeutic molecules [427]. Investigators have used mPEG-SH and a positively charged peptide (RRLAC) to alter rod-like Au NPs to modify the surface chemistry of Au NPs. When conjugated with RRLAC, the PEG on Au NP surfaces can help to stabilise and elude aggregation. Supplementary conjugate photosensitizers (aluminium phthalocyanine tetrasulfonate, AlPcS4) can be applied to the positively charged RRLAC by charge-charge 
forces. The disclosure of $810 \mathrm{~nm}$ laser irradiation will quickly produce a photothermal effect, as well as an indication of the reactive AlPcS4 discharge. The second $670 \mathrm{~nm}$ laser irradiation exposure generates the output for photodynamic therapy of a singlet oxygen generator (SOG). Joining the photo-thermal therapy and photo-dynamics therapy can expressively increase anti-cancer therapeutic properties. To improve the loading proficiency of therapeutic molecules, mesoporous silica $\left(\mathrm{SiO}_{2}\right)$ was used to increase the surface chemistry of Au NPs [428] to boost the loading skills of therapeutic molecules. As a new cancer theranosis agent [429] investigators have developed $\mathrm{SiO}_{2}$-coated rod-like $\mathrm{Au} \mathrm{NPs}$ $\left(\mathrm{Au} @ \mathrm{SiO}_{2}\right)$. The $\mathrm{SiO}_{2}$ large surface area and large cavity will greatly advance the loading of chemotherapy medications.

\subsection{Other Applications}

\subsubsection{Gold Nanoparticles as a Catalyst}

The gold nanoparticles are a good catalyst and have brings about the catalysis of many dyes degradation wether the gold nanoparticles synthesized by the chemical method or physical method. The gold nanoparticle is a precious metal for coinage, jewelry and other arts since antiquity. Chemically, gold is a transition metal gorgeous in coordination numbers. However, relative to other metals Bulk $\mathrm{Au}$ is a renowned chemically inert material and has been reflected as an unwell active catalyst in numerous reactions. Though $\mathrm{Au}$ NPs exhibit high catalytic characteristics for several reactions [430,431]. The finding of the outstanding catalytic properties of Au NPs was described for the first time by Haruta et al. in 1989, who showed that Au NPs can be very active in the elevation of the oxidation of carbon monoxide (CO) at little temperature [432]. Succeeding this discovery, a sample of aerobic oxidation reactions catalyzed by Au NPs have been described. For example, the oxidation of alcohols to aldehydes [433], carboxylic acids [430] or esters [434], the oxidation of aldehydes to esters [30] or acids [435], epoxidations of olefins and the oxidation of amines to amides [436] have been examined. Newly, the catalytic use of Au NPs in careful hydrogenation has been extensively testified as well [437]. Specifically, it was revealed that with the incidence of a Au NP catalyst, replaced anilines and connected products can be formed proficiently at low temperature from their nitrogenated compounds, which are normally toxic organic by-products formed unenviably throughout the industrial developed method of chemicals, such as agrochemicals, dyes and pharmaceuticals [438]. For instance, 4-nitrophenol (4-NP), a toxic nitro acromatic chemical, has a postponed interaction with blood and then forms methaemoglobin tempting methemoglobinemia, potentially producing cyanosis, confusion and unconsciousness to human body. When swallowed, it causes abdominal pain and vomiting. Instead, 4-aminophenol (4-AP), a hydrogenation yield of 4-NP, is a very beneficial material which can be employed as an significant intermediate in pharmaceutical preparation of analgesic and antipyretic drugs [439] as antioxidant in plastics fabrication [438]. More significantly, this reaction is highly helpful for the moving environmental pollutants into beneficial chemicals. With $\mathrm{Au}$ NPs, unsaturated chemicals can also be selectively hydrogenated. For example, acetylene and 1, 3-butadiene can be moderately hydrogenated into ethylene [440] and butenes correspondingly. The chief benefit of Au NP catalysts is their capability to selectively catalyze the hydrogenation of the $\mathrm{C}=\mathrm{O}$ group of $\alpha, \beta$-unsaturated aldehydes manufacturing allyl-type unsaturated alcohols [441]. The reduction of 4-NP to 4-AP on Au NPs with sodium borohydride $\left(\mathrm{BH}_{4}^{-}\right)$in aqueous solution has also been thoroughly employed as a style reaction to inspect the catalytic presentation of noble metal NPs, specially Au NPs synthesized from the reduction of auric acid via wet chemical methods [442]. For instance, Pal and coworkers found that a reduction in Au NP size primes to the increase of decrease rate [443]. The alteration of Au NP surfaces with diverse functional groups testified that the surface structure also mainly effects the catalytic characteristics of Au NPs [444].

The catalytic mechanism for reduction of 4-NP by Au NPs@vesicles catalyst to form 4-AP in the presence of $\mathrm{NaBH}_{4}$ is shown in Figure 53. Here in the mechanism, when $\mathrm{Au} \mathrm{NPs} @$ vesicles was used for catalytic reduction, $\mathrm{BH}_{4}{ }^{-}$and 4-NP are first spread from 
aqueous solution to the Au surface and then the Au NPs on vesicle function as catalysts for the transfer of electrons from $\mathrm{BH}_{4}{ }^{-}$to nitrophenols. Too much borohydride $\mathrm{BH}_{4}{ }^{-}$ion from $\mathrm{NaBH}_{4}$ adsorbed and then shifted a hydride to the surface of Au NPs@vesicles, subsequent in the improvement of Au-hydride bonds. At the same time, adsorption of 4-NP ion onto the Au NPs@vesicles takes place. The nitro group of 4-NP molecules slopes to attract hydrogen and electrons from the Au-hydride complex [445]. Finally, 4-aminophenol as the final product is formed via various steps of hydrodeoxygenation reactions.

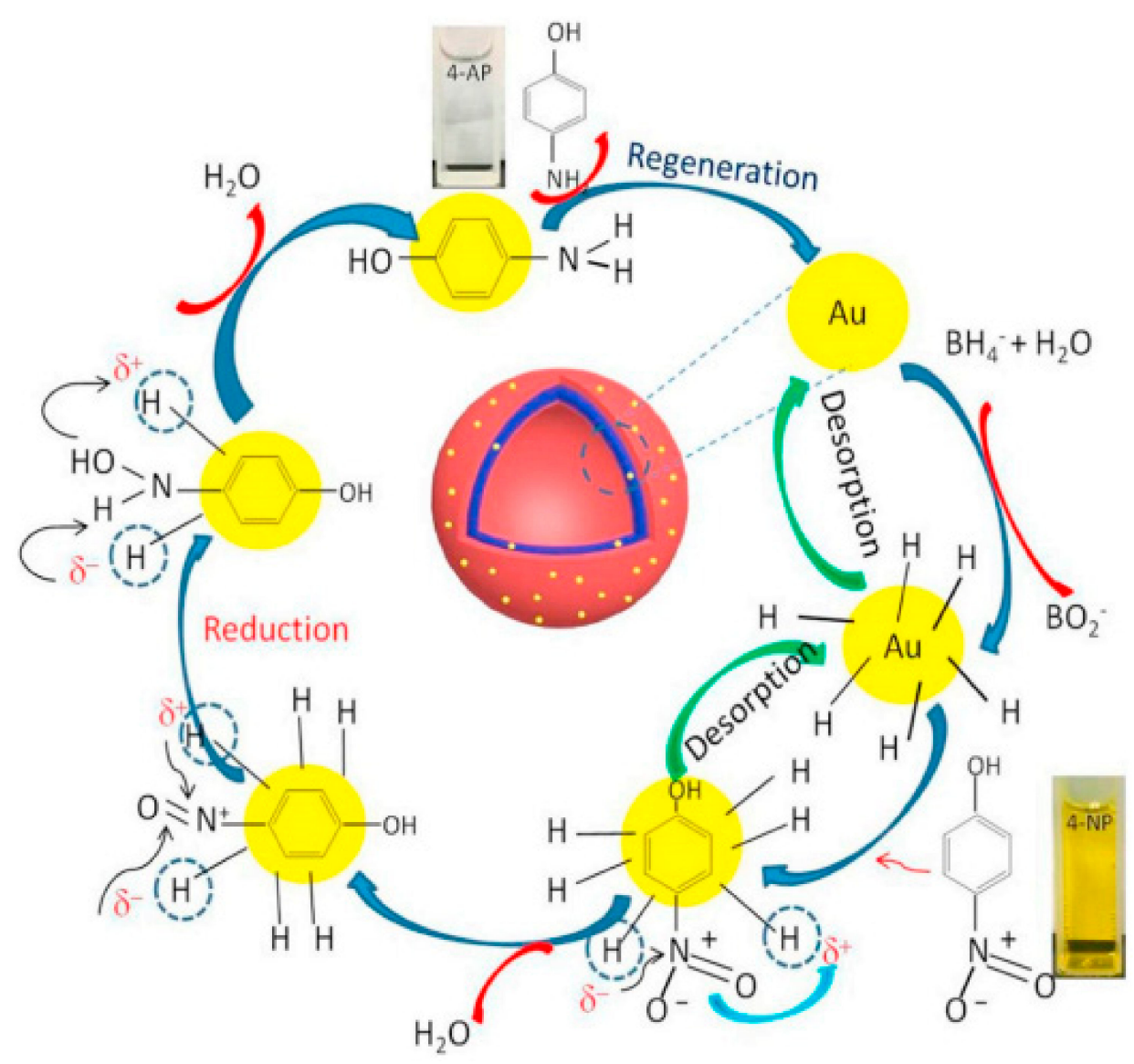

Figure 53. The catalytic mechanism for reduction of 4-NP by Au NPs@vesicles catalyst. Reproduced with permission from Elsevier [446], Copyright 2019, Materials Science \& Engineering C.

A brief overview of the catalytic activities of the gold nanoparticles, studied so far, is summarized here. Sujoy et al. [447] synthesized gold nanoaprticles, sizes ranging from 5 to $65 \mathrm{~nm}$, by green synthesis method of protein extract of Rhizopus oryzae and produced the gold nano-bioconjugates (AuNBC). The synthesized gold nano-bioconjugates (AuNBC) showed excellent stability of the different parameters like ionic strength, $\mathrm{pH}$ and temperature arose from the electrostatic repulsion of the negative charge of the conjugate proteins. Catalytic reduction of p-NP by NaBH4 in presence of AuNBCs and time-dependent UVVis absorption are shown in Figure 54. These gold nano-bioconjugates (AuNBC) have showed excellent catalytic activity compared to that observed through the reduction of p-nitrophenol by borohydride and Au NPs synthesized through the conventional methods. It was also found that, without AuNBC addition, the absorbance at $400 \mathrm{~nm}$ did not change with time, showing that the p-NP reduction did not proceed in lack of catalyst. The good catalytic performance of Au NPs could be assigned to the additional stability and functionalization provided by the protein extract used as biological reducing agent. 

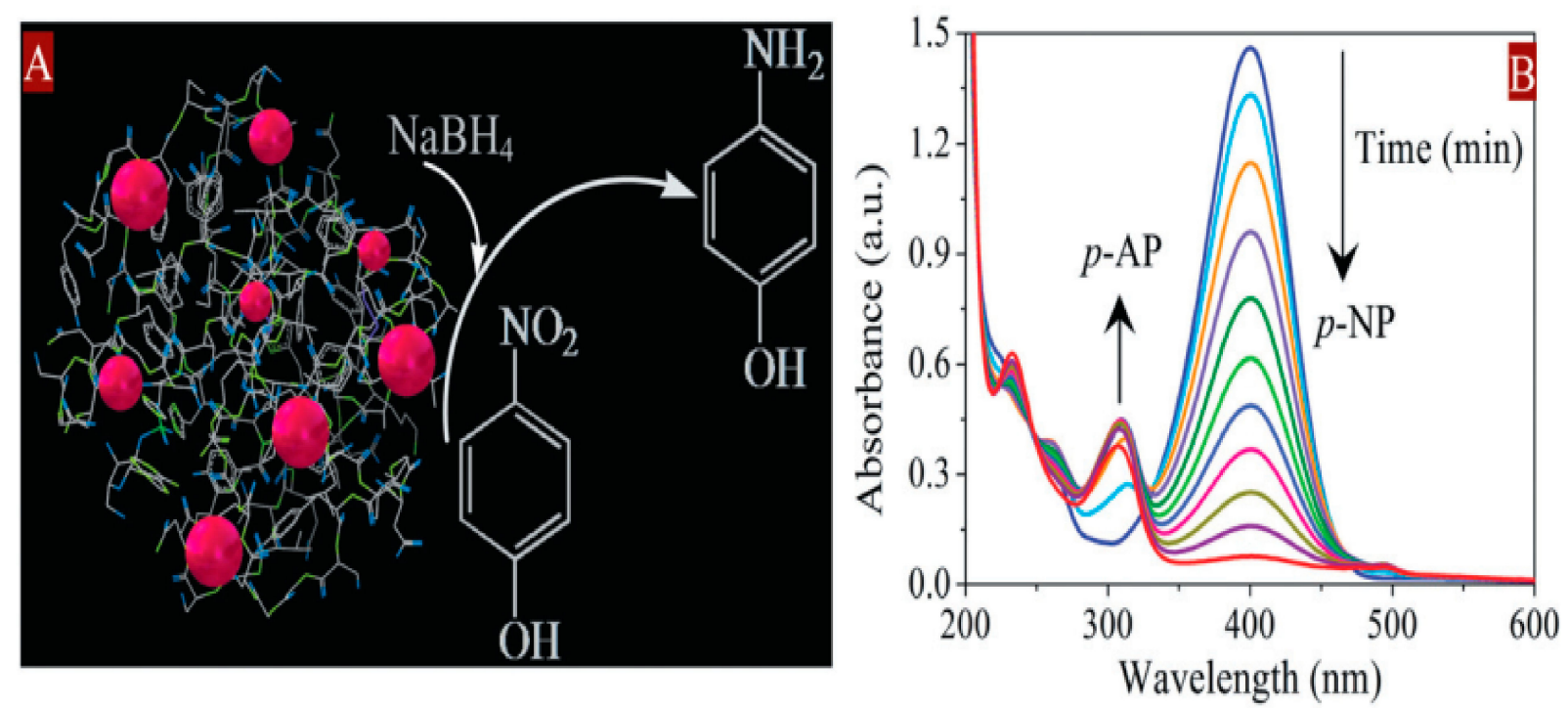

Figure 54. Catalytic activity of AuNBC (A) for p-NP reduction. Time dependent UV-vis absorption spectra (B) for catalytic reduction of $\mathrm{p}-\mathrm{NP}$ by NaBH4 in presence of AuNBCs. Condition used throughout: $[\mathrm{p}-\mathrm{NP}]=1.0 \times 10^{-4} \mathrm{M}$; $[\mathrm{NaBH} 4]=1.0 \times 10^{-2} \mathrm{M}$; [AuNBCs] $=0.0101 \mathrm{mM}, \mathrm{T}=20^{\circ} \mathrm{C}$. Reproduced with permission from Green Chem. [447], Copyright 2012, RSC.

Mamatha et al. [448] studied the application of Au NPs toward the catalytic degradation of organic dyes. An overall observation of their results indicated that the gold nanoaprticles ( $\mathrm{Au}$ NP B. sensitivum), prepared via green synthesis process using the leaves of the plant Biophytumsensitivumas with $1 \mathrm{mM}$ solution of $\mathrm{HAuCl}_{4} \cdot 3 \mathrm{H}_{2} \mathrm{O}$ in 1:9 ratio, showed good catalytic activities toward the degradation of organic azo dye, congo red in water under ordinary experimental setup. This better catalytic imfact can not only be due to their smaller size (size $<100 \mathrm{~nm}$ ) but also to their better morphology. Likewise, Indramani et al. [449] synthesised the gold nanoparticles while using extracts of Sansevieria roxburghiana leaf and the chloroauric acid as reducing and stabilizing agents. The particles so obtained were checked for their catalytic efficiency by confrirming fom the degradation of degradation of organic pollutants such as 4-nitrophenol, acridine orange, congo red, bromothymol blue, phenol red and methylene blue. It was found that the gold NPs showed good efficiency toward various pollutants in water. Further, an overall investigation of the previous work shows that Au NPs prepared through the green/biological approach have better performance as compared those prepared while conventional chemical procedures.

\subsubsection{Gold Nanoparticles as A Photocatalyst}

Typically, the photocatalytic activity can be estimated employing the external quantum yield $\left(\phi_{\mathrm{ex}}\right)$ which can be calculated as:

$$
\phi_{\mathrm{ex}}=[\mathrm{n} \times(\text { number of product molecules }) / \text { number of incident photons }]
$$

where $n=2$ for the HER (product $=\mathrm{H}_{2}$ ) and the 2 electron ORR (product $=\mathrm{H}_{2} \mathrm{O}_{2}$ ) and $n=4$ for the WOR $\left(\mathrm{O}_{2}\right)$. In the light of the previous literature, it can be studied that Au NPs in their non-composities/supported are rarely used as photocatalyst; however, an appreciable amount of work shows that Au NPs in their composities/supported version with other materials are studied for their photocatalytic purposes as well.

\section{Au NPs for Water Splitting}

Moskovits and his coworkers were the first to display plasmonic overall water splitting while employing $\mathrm{TiO}_{2}$ capped $\mathrm{Au}$ nanorods with $\mathrm{Pt}$ as the hydrogen evolution catalyst on $\mathrm{TiO}_{2}$ and a Co-based oxygen evolution catalyst on $\mathrm{Au}\left(\phi_{\mathrm{ex}}=0.1 \%\right)$ [450]. The $\mathrm{Au} / \mathrm{TiO}_{2}-$ $\mathrm{NiOx}$ plasmonic photocatalyst has also been described to be proficient of splitting water 
with $\phi_{\text {ex }}=0.013 \%$ at $\mathrm{hv}=2.1 \mathrm{eV}$ [451]. As revealed in the scheme 2 (Figure 55), the low CB minimum of $\mathrm{TiO}_{2}\left(\mathrm{ECBM}=-4.1 \mathrm{eV}\right.$ for rutile $\mathrm{TiO}_{2}$ and $3.9 \mathrm{eV}$ for anatase $\mathrm{TiO}_{2}$ at $\mathrm{pH} 7$ vs. vacuum level) which is inadequate for the $\mathrm{HER}(\mathrm{ECBM}=-4.02 \mathrm{eV}$ at $\mathrm{pH}$ 7) is mostly accountable for the partial proficiencies. Instead, $\mathrm{CdS}$ takes a plentiful greater $\mathrm{CB}$ minimum (ECBM = -3.28 eV) [452]. Peculiar asymmetrical nanohybrids mentioned to as half-cut $\mathrm{Au}$ (core) and $\mathrm{CdS}$ (shell) nano eggs without and with a hetero epitaxial junction (HC-Au@CdS and HC-Au@\#CdS) were prepared employing a modified photodeposition technique [453]. Water splitting experiments were carried out under irradiation of red-light [454]. Figure 56a,b displays the contrast of the photocatalytic activity of the nanohybrids and the single components. In this circumstance, the Au particle size was secured at $5.5 \mathrm{~nm}$. Au and CdS are nearly quiet; however, the physical mixture displays little action, non-heteroepitaxial junction HC-Au@CdS had a much greater action. The photocatalytic activity enhances by about one-order of magnitude with an increase in the size of Au particle from $5.5 \mathrm{~nm}$ to $12.1 \mathrm{~nm}$ and $\phi_{\mathrm{ex}}=0.24 \%$ at $\mathrm{h} v=1.9 \mathrm{eV}$ has been attained; this behavior can be seen from Figure 56b. Additionally, even after three recurrences of the 3 day reaction, no decay in the action was detected with the constant stoichiometric production of $\mathrm{H}_{2}$ and $\mathrm{O}_{2}$.

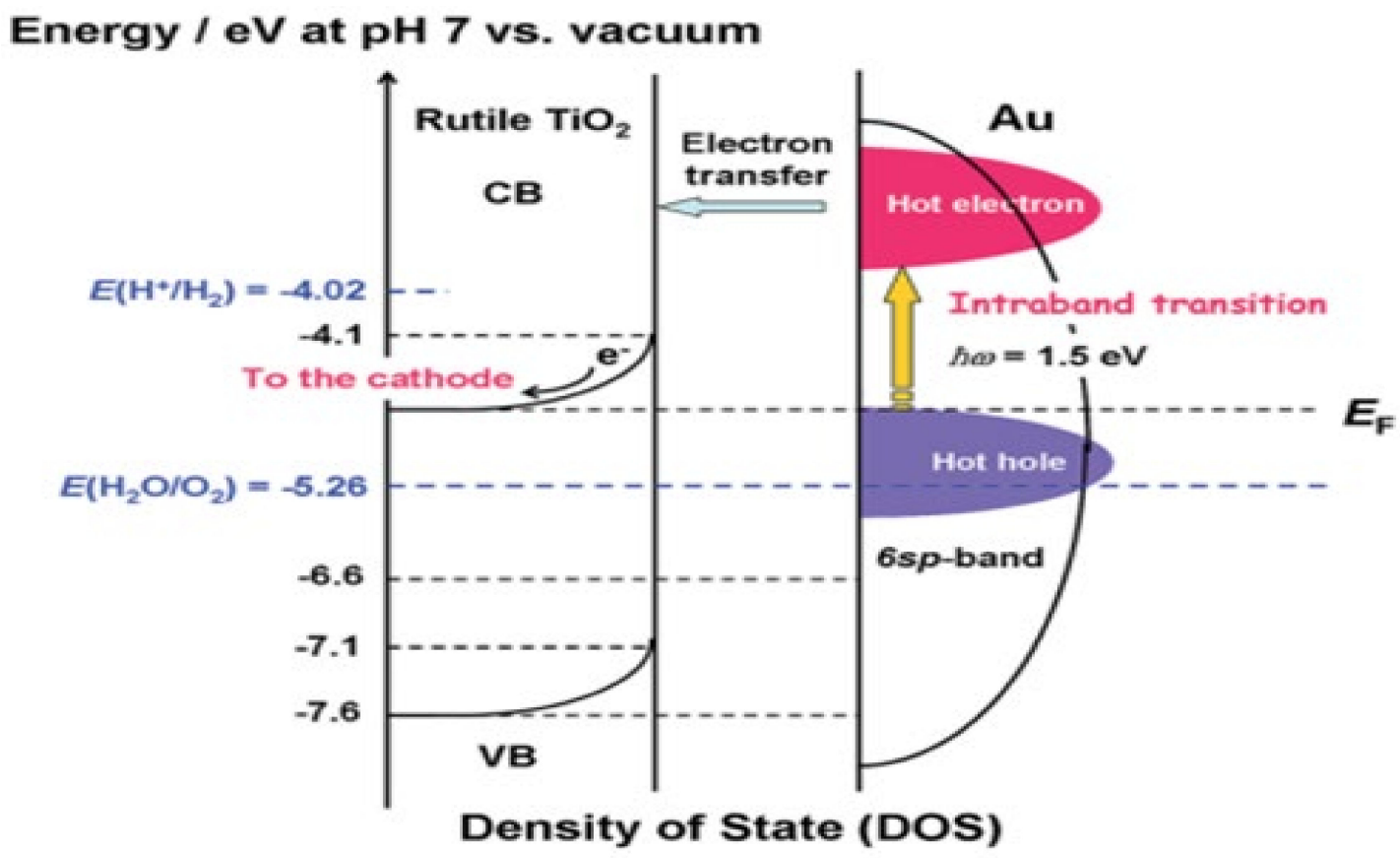

Figure 55. Schematic representation of the energy diagram of the $\mathrm{Au} / \mathrm{TiO}_{2}$ plasmonic electrode with the density of states of $\mathrm{TiO}_{2}$ and energy distribution of the hot carriers in Au NPs with the intraband transition through the surface plasmon decay. Reproduced with permission from Nano scale Adv. [455], Copyright 2019, RSC.

Figure 57 demonstrates the basic reaction scheme of water splitting by the HCAu@\#CdS plasmonic photocatalyst. HCAu@\#CdS capably absorbs sunlight. The hotelectrons produced through the LSPR excitation can be efficiently inserted into the CB of $\mathrm{CdS}$ over the large area and high-quality junction. The high-energy electrons in the CB of $\mathrm{CdS}$ allow a smooth HER, while the hot holes absent in Au NPs oxidize water with the help of the electrocatalytic activity for the WOR [456]. Significantly, selective excitation of the Au NP-LSPR overwhelms the photodissolution of CdS [457] so far hindering its employ as a water splitting photocatalyst. Tan and co-workers have newly synthesized a Pt NP-loaded $\mathrm{TiO}_{2}$ hierarchical nano-design $\left(\mathrm{Pt} / \mathrm{TiO}_{2}-\mathrm{HA}\right)$, presenting a great level of visible-light action for overall water splitting $\left(\phi_{\mathrm{ex}}=0.23 \%\right.$ at $\left.\mathrm{hv}=2.25 \mathrm{eV}\right)$. These authors also suggested the $\mathrm{HET}$ mechanism for the $\mathrm{Pt} / \mathrm{TiO}_{2}-\mathrm{HA}$-photocatalyzed water splitting phenomenon. 
a)

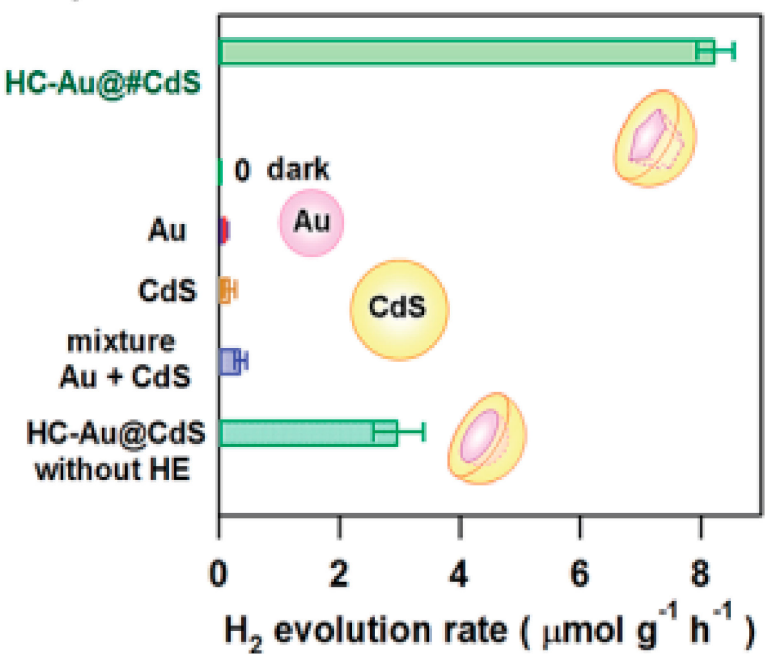

b)

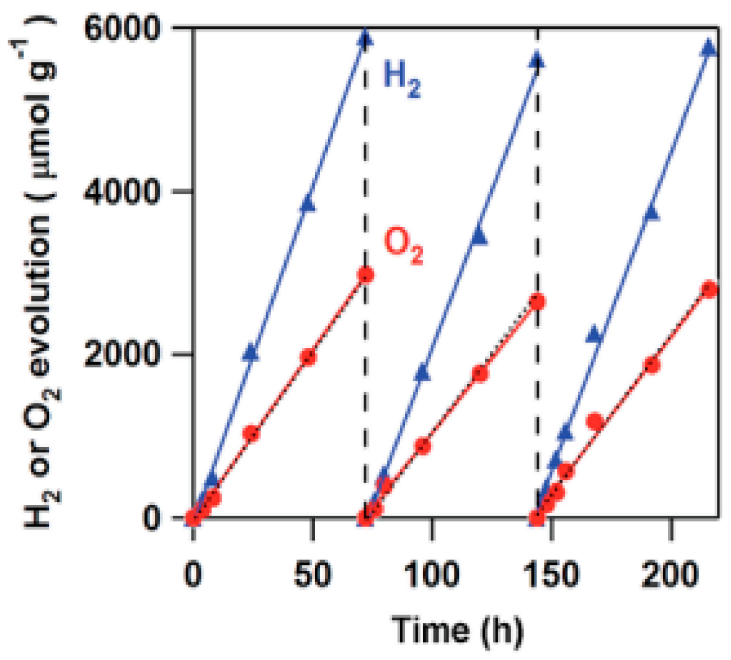

Figure 56. (a) The comparison of the $\mathrm{H}_{2}$ evolution rate for $\mathrm{HC}-\mathrm{Au}\left(\mathrm{d}_{\mathrm{Au}}=5.5 \mathrm{~nm}\right) @ \# \mathrm{CdS}(/ \mathrm{CdS}=1.9 \mathrm{~nm}), \mathrm{Au}$ colloid, CdS and a mixture of $\mathrm{Au}$ and $\mathrm{CdS}$ under red-light illumination $(\lambda \mathrm{ex}=640 \mathrm{~nm})$ or dark conditions. (b) Repeated water splitting by $\mathrm{HC}-\mathrm{Au}\left(\mathrm{d}_{\mathrm{Au}}=12.1 \mathrm{~nm}\right) @ \# \mathrm{CdS}(/ \mathrm{CdS}=2.1 \mathrm{~nm})$ under red-light illumination. Figures are taken from ref. Reproduced with permission from Nano scale Adv. [454], Copyright 2019, RSC.



\section{Electrocatalytic activity} for WOR

Figure 57. Schematic representation of water splitting by the $\mathrm{HCAu} @ \mathrm{AdS} / \mathrm{TiO}_{2}$ plasmonic photocatalyst. Reproduced with permission from Nano scale Adv. [455], Copyright 2019, RSC.

Role of Au NPs in Redox/Chemical synthesis

$\mathrm{Au} / \mathrm{TiO}_{2}$ plasmonic photocatalysts have been used to numerous significant oxidations [458]. This is possibly since the electrocatalytic activity of Au NPs for the reduction reaction cannot be used in the usual HET-type $\mathrm{Au} / \mathrm{TiO}_{2}$ plasmonic photocatalyst, where $\mathrm{Au}$ and $\mathrm{TiO}_{2}$ acted as oxidation and reduction positions, respectively. It was revealed that visible-light irradiation of small- $\left(\mathrm{d}_{\mathrm{Au}} \approx 2 \mathrm{~nm}\right)$ and large- $\left(\mathrm{d}_{\mathrm{Au}} \approx 10 \mathrm{~nm}\right)$ Au NP-loaded $\mathrm{TiO}_{2}$ stated to as bimodal (BM)-Au/ $\mathrm{TiO}_{2}$ prompts the interfacial electron transfer from small $\mathrm{Au}$ NPs to large $\mathrm{Au}$ NPs via the $\mathrm{CB}$ of $\mathrm{TiO}_{2}$ [459]. This phenomenon was efficient in relations of the entropic energetic force for the interfacial electron transfer [436]. A comparison showed that $\mathrm{BM}-\mathrm{Au} / \mathrm{TiO}_{2}$ shows much greater activity than small- $\mathrm{Au} / \mathrm{TiO}$ and large- $\mathrm{Au} / \mathrm{TiO}_{2}$. It is well known that $\mathrm{H}_{2} \mathrm{O}_{2}$ strongly adsorbs on $\mathrm{TiO}_{2}$ to form a surface com- 
plex (Tis- $\mathrm{OH}+\mathrm{H}_{2} \mathrm{O}_{2}$ /Tis- $\mathrm{OOH}+\mathrm{H}_{2} \mathrm{O}$ ). The surface complex experiences reductive decay by the CB-electrons in $\mathrm{TiO}_{2}$ in a manner: Tis- $\mathrm{OOH}+2 \mathrm{H}++2 \mathrm{eCB} / \mathrm{Tis}-\mathrm{OH}+\mathrm{H}_{2} \mathrm{O}$ [460]. Formerly, it is also significant to overwhelm this breakdown way to enhance the income of $\mathrm{H}_{2} \mathrm{O}_{2}$. An operative method is the surface-fluorination of $\mathrm{TiO}_{2}$ [461,462] permitting the generation of $\mathrm{H}_{2} \mathrm{O}_{2}$ at a millimolar level under UV-light irradiation. [463]. Then, the consequence of the surface modification of $\mathrm{BM}-\mathrm{Au} / \mathrm{TiO}_{2}$ with carbonate ions (BM$\mathrm{Au} / \mathrm{TiO}_{2}-\mathrm{CO}_{3}{ }^{2-}$ ) on the photocatalytic activity was inspected. It was also found that the surface modification radically enhances the photocatalytic activity and the $\phi_{\text {ex }}$ reached $5.4 \%$ at $\mathrm{hv}=2.3 \mathrm{eV}$. In the $\mathrm{H}_{2} \mathrm{O}_{2}$ preparation from water and $\mathrm{O}_{2}$ employing semiconductor photocatalysts, a $\phi_{\text {ex }}$ value of $5.4 \%$ at $\mathrm{h} v=2.95 \mathrm{eV}$ was testified for $\mathrm{Au} / \mathrm{BiVO}_{4}$ [464]. Significantly, Shiraishi and coworkers have found that $\mathrm{g}-\mathrm{C}_{3} \mathrm{~N}_{4}$ owns an tremendously high discrimination of $90 \%$ for electrochemical $\mathrm{H}_{2} \mathrm{O}_{2}$ creation [465], and the proficiency of photocatalytic $\mathrm{H}_{2} \mathrm{O}_{2}$ preparation was significantly enhanced by employing it as the photocatalyst $\left(\phi_{\mathrm{ex}}=2.6 \%\right.$ at $\left.\mathrm{h} v=2.95 \mathrm{eV}\right)$ [466]. In a porous defective $\mathrm{g}-\mathrm{C}_{3} \mathrm{~N}_{4}$ photocatalytic system, a tremendously high $\phi_{\text {ex }}$ value of $16 \%$ was attained in the company of 2-propanol as an electron donor at $\hbar \mathrm{u}=3.26 \mathrm{eV} .13$ The great photocatalytic activity of $\mathrm{BM}-\mathrm{Au} / \mathrm{TiO}_{2}$ for $\mathrm{H}_{2} \mathrm{O}_{2}$ preparation from water and $\mathrm{O}_{2}$ can be reorganised as given in Figure 58. This type of mechanism can be applied to other redox synthesis. Visible-light irradiation of $\mathrm{BM}-\mathrm{Au} / \mathrm{TiO}_{2}$ contributes rise to the net electron transport from small Au NPs to large Au NPs, gathering electrons and holes in large and small Au NPs, correspondingly. Consequently, water is oxidized on small Au NPs, while the two-electron ORR happens on large Au NPs. Finally, the greater photocatalytic activity of $\mathrm{BM}-\mathrm{Au} / \mathrm{TiO}_{2}$ for $\mathrm{H}_{2} \mathrm{O}_{2}$ preparation can stem from the effective charge departure via the interfacial electron transfer from small Au NPs to large $\mathrm{Au}$ NPs, the previous one shows outstanding electrocatalytic activity for the WOR and the little catalytic activity of the small and large Au NPs for $\mathrm{H}_{2} \mathrm{O}_{2}$ breakdown [467]. Further, the surface modification with $\mathrm{CO}_{3}{ }^{2-}$ ions of $\mathrm{BM}-\mathrm{Au} / \mathrm{TiO}_{2}$ is effective in suppressing the reductive decomposition of $\mathrm{H}_{2} \mathrm{O}_{2}$ to increase its yield. From a viewpoint of organic synthesis, $\mathrm{BM}-\mathrm{Au} / \mathrm{TiO}_{2}$ has also paved a way for the application of plasmonic photocatalysts in reductive chemical transformations. For instance, $\mathrm{BM}-\mathrm{Au} / \mathrm{TiO}_{2}$ showed a great level of visible-light activity for the one-step preparation of azobenzenes from nitrobenzenes at $25{ }^{\circ} \mathrm{C}$ with a high yield of $>95 \%$ and selectivity $>99 \%$, whereas unimodal $\mathrm{Au} / \mathrm{TiO}_{2}$ is photocatalytically less active [459].

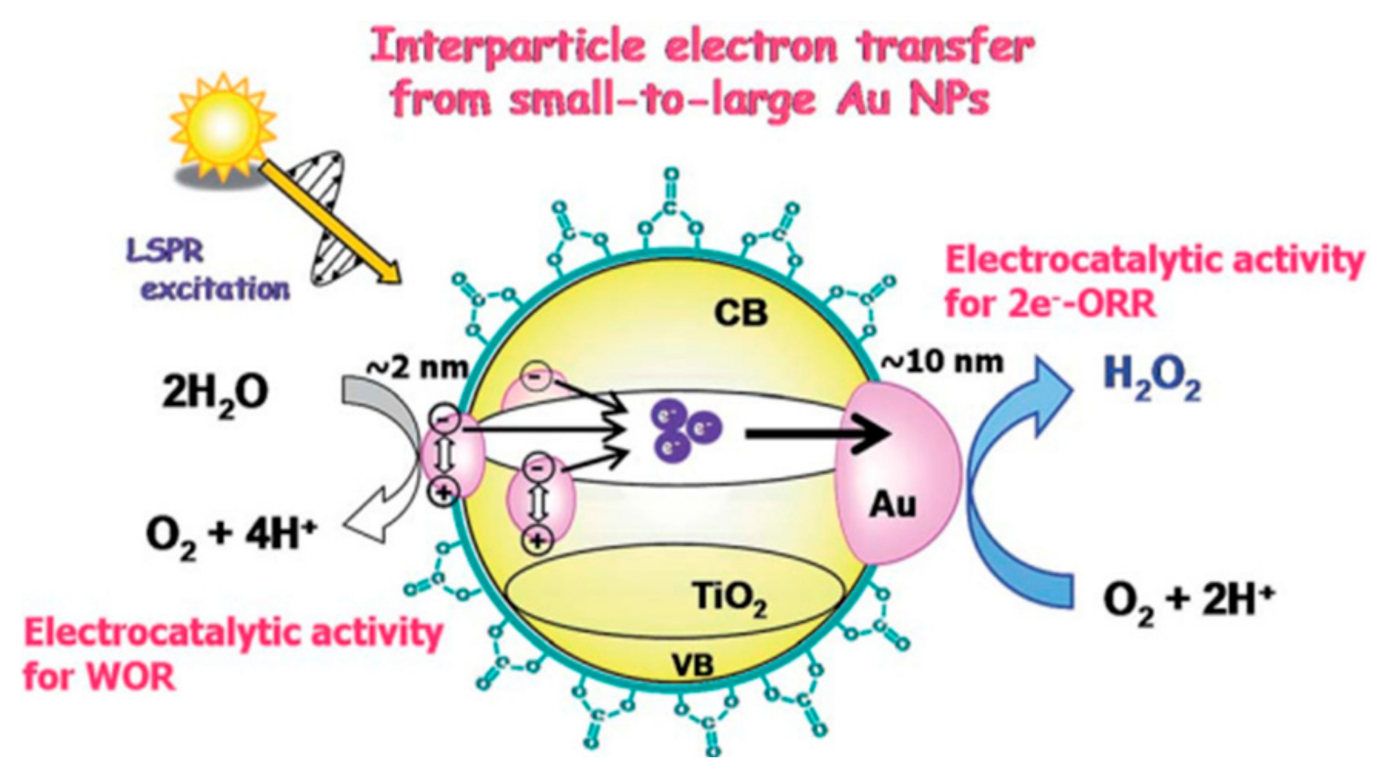

Figure 58. A schematic representation of $\mathrm{H}_{2} \mathrm{O}_{2}$ from water and $\mathrm{O}_{2}$ by the $\mathrm{BM}-\mathrm{Au} / \mathrm{TiO}_{2}$ plasmonic photocatalyst. Reproduced with permission from Nano scale Adv. [455], Copyright 2019, RSC. 


\subsubsection{Use of Au NPs in Sensing}

The communication of light at the surface of the noble metal film stimulates surface electromagnetic waves and sets them to resonate with incident light wave, subsequent in the absorption of the light. This phenomenon is recognized as surface plasmon resonance (SPR) [468] and rest on the refractive index of the interfacial region. Metal nanoparticles, such as gold [469] and silver, display localized surface plasmon resonance (LSPR) at specific instance wavelengths, producing strong light scattering and the arrival of intense surface plasmon absorption bands. The intensity and frequency of the absorption band is typical of the specific metal nanoparticles and extremely reliant on their size and shape, as well as the surrounding environment [470] Using this phenomenon, many LSPR-based chemical and biological sensors have been advanced [471]. However, many biosensors have been advanced by means of silver nanoparticles [472]. Here, we summarize some of sensing properties of the Au NPs reported elsewhere [473].

\section{Sensors Based on Change in LSPR Absorption of Au NPs}

The overall value behind LSPR-based sensors is the wavelength shift in the LSPR spectrum rising from local dielectric variations produced by analyte adsorption. Some studies based on LSPR have been conducted both in solution phase $[474,475]$ and on surfaces coated with nanoparticle monolayers [476,477]. Solutions phase study shows that absorption maxima of LSPR was red shifted when Au NPs functionalized with monoclonal antibodies interrelated with analytes. Furthermore, the wavelength shift was made to be proportional to the quantity of ligands [478]. Likewise, most of Au NP-based SPR sensors were reported by arresting nanoparticles onto surfaceof solids [479]. The addition of Au NPs onto the sensing surface delivers an actual method to enhance the sensitivity of SPR sensors is because of the high dielectric constants of Au NPs and the electromagnetic coupling between Au NPs and the metal film on the surface. For instant, a gold film-coated chip was employed to identify dopamine in nano molar concentration by immobilizing an MIP gel with embedded Au NPs [480]. Numerous substrates, such as quartz, optical fibers, ITO glass and sol-gel matrix, have been employed for supports for Au NPs, permitting the finding of many analytes, such as human serum albumin, BSA, human IgG, streptavidin, interleukin-1 $\beta$ and propanethiol [481]. Currently, Au NPs encapsulated by hydroxyl/thiolfunctionalized fourth production PAMAM dendrimer were immobilized onto maleimide terminated SAMs to notice insulin. The subsequent Au NP-modified dendrimer surface has high stability and increased sensitivity with a recognition limit of $0.5 \mathrm{pM}$. These sensors showed good activity by analyzing human serum samples from normal and diabetic patients with decent association to standard approaches [482]. The aggregation of $\mathrm{Au}$ NPs brings an alteration to surface absorption band that give rise to a visible color change. Exploiting this value, Mirkin et al. advanced a colorimetric sensor for DNA hybridization examination employing oligonucletide functionalized Au NPs both in dispersions and on surface. Other SPR grounded sensors employing aggregation of $\mathrm{Au}$ NPs have been described drawing attention to proteins (via antigen-antibody or biotinstreptavidin interaction) and lectin. Au NPs of mediated SPR signal amplifcation have been employed to enhance the spreading SPR spectroscopic signals and hence improved sensor sensitivity $[483,484]$. The signal amplification was described by the electronic coupling contact of the spreading surface plasmons with localized surface plasmons of Au NPs and be dependent on many factors such as size, shape and the distance from the metal fabricating [485].

\section{Sensing of Proteins}

Protein sensing via antigen-antibody interaction can be noticed using Au NP-amplified SPR phenomena. For example, Natan et al. have described a Au NP-enhanced SPR immune sensing system by means of either antigen or secondary antibody functionalized Au NPs as signal enhancers [486]. In an instance of this sandwich approach, a gold film coated with Fc specific monoclonal goat antihuman $\operatorname{IgG}(\alpha-h-\operatorname{Ig} G(\mathrm{Fc}))$ produces a small plasmon 
shift upon addition of human IgG and the second free antibody. The plasmon shift, though, enhances 28-fold relative to an unamplified examine when the secondary free antibody is substituted by an electrostatic conjugate between Au NPs and $\alpha$-h-IgG(Fc). Using this technique, picomolar recognition of human $\operatorname{IgG}$ is attained. Likewise, numerous competitive and sandwich immune assays have been advanced employing Au NP-increased SPR signals to notice human tissue inhibitor of metalloproteinases-2, [487] antiglutamic acid decarboxylase antibody, allergen, TNT, human IgE and testosterone [488]. The sensitivity of these assays can be increased employing fluorescence-labeled antibodies decorated with $\mathrm{Au}$ NPs, gives rise to the method called localized surface plasmon resonance coupled fluorescence fiber optic sensor [489,490].

Sensing of Oligonucleotides for Inhirtence Tracing

The sensitivity of oligonucleotide detection can be amended by employing Au NP amplified SPR [491]. Keating et al. advanced a sandwich method where 12-mer oligonucleotides were first related covalently onto a gold substrate shadowed by hybridization of one-half of the target DNA molecules. Then, an arrangement opposite to the other half of the target was added with or without tagging of Au NPs. The Au NP-tagged surface confirmed a 10-fold enhance in an angle shift, concomitant with a 1000-fold development in sensitivity and a $\sim 10 \mathrm{pM}$ recognition limit for the target 24-mer oligonucleotide [492]. For example, Zhou et al. revealed that an intermediate carboxylated dextran layer between gold film and the immobilized DNA molecules efficiently removes the nonspecific adsorption of oligonucleotide functionalized Au NPs (see Figure 59); this method can result in the detection of 39-mer DNA at femto molar level [493]. The SPR measurements were carried out by injecting the oligonucleotide functionalized Au NPs into the flow cell housing sensors covered with various duplexes or capture probes. The intermediate dextran layer reduces the nonspecific adsorption of Au NPs, improving detection sensitivity. In an illustrative study, real time multicolor DNA detection has attained developing Au NP-amplified diffraction, where ssDNA modified Au NPs and micropatterned chemoresponsive diffraction gratings were employed to interrogate simultaneously at multiple laser wave lengths [494].

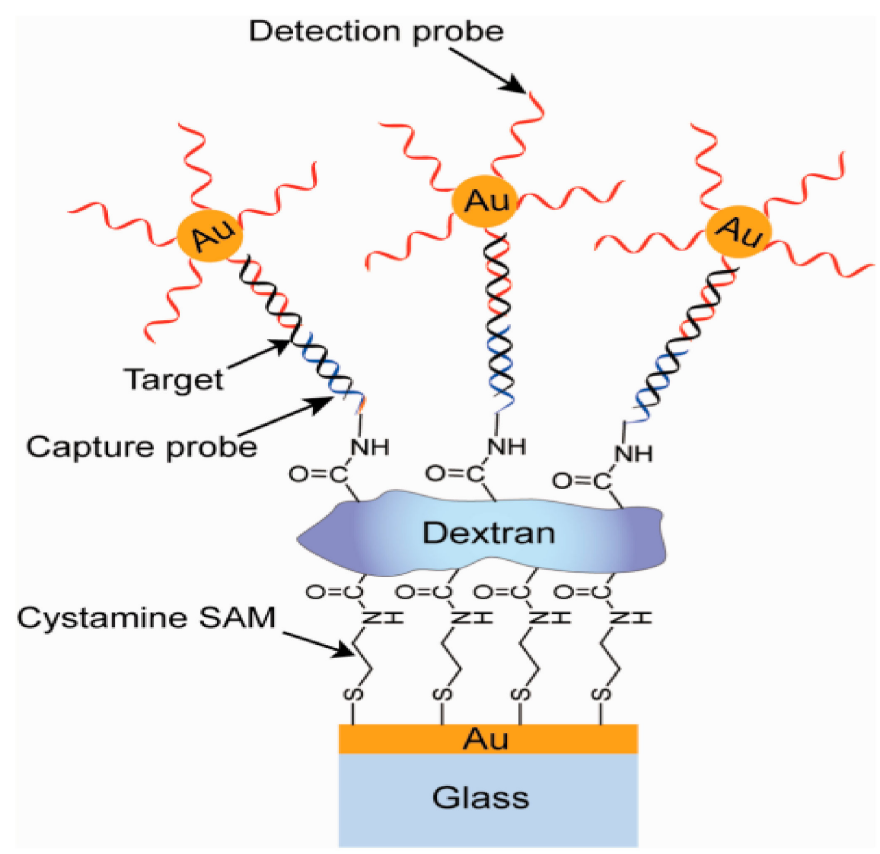

Figure 59. Schematic representation of sandwich DNA detection assay via Au NP mediated SPR signal amplification. Reproduced with permission from Anal. Biochem. [493] Copyright 2006, Elevier B.V. 


\section{Biosensors Bassed on Au NP SPR Scattering Approach}

The plasmon resonance scattering phenomenon of Au NPs can offer a beneficial instrument for sensor design by inspecting the variations in LSPR absorption of Au NPs [495]. The plasmon scattering of $36 \mathrm{~nm}$ diameter Au NPs is 10-100 times stronger than dyes or quantum dots. Using this nano-scale phenomena, numerous groups have advanced immune assays to detect human IgG [496], kanamycin and lysozyme in human urine [497]. For instant, Ren and colleagues have advanced an extremely careful and sensitive similar immune assay and DNA hybridization assay using plasmon scattering of single Au NP evaluation. The sandwich immuneo assay was employed to notice cancer biomarkers for example CEA, AFP in femtomolar range and aptamer recognition for thrombin as low as $2.72 \mathrm{pM}$ [498]. Recently, Ling et al. described an LSPR light scattering sensor for $\mathrm{Ag}^{+}$with unmodified Au NPs using the specific respect characteristic of $\mathrm{Ag}^{+}$with a cytosine-cytosine gap base pair. The addition of $\mathrm{Ag}^{+}$eliminates the oligonucleotide from the Au NP surface producing aggregation concomitant with dramatic increase of LSPR scattering strength The LSPR light scattering intensity was proportional to concentration of $\mathrm{Ag}^{+}$with a limit of detection of $62 \mathrm{nM}$ [499]. El Sayed et al. confirmed a biosensor method employing SPR scattering images and SPR absorption spectra from anti-EGFR functionalized Au NPs (Figure 60) for the diagnosis of oral epithelial cancer cells in vitro [500]. They oserved that anti-EGFR functionalized Au NPs bind 600\% stronger to oral malignant cells HOC 313 clone 8 and HSC 3 than normal cell HaCaT; this results in a sharper SPR absorption band with a clear red shift.
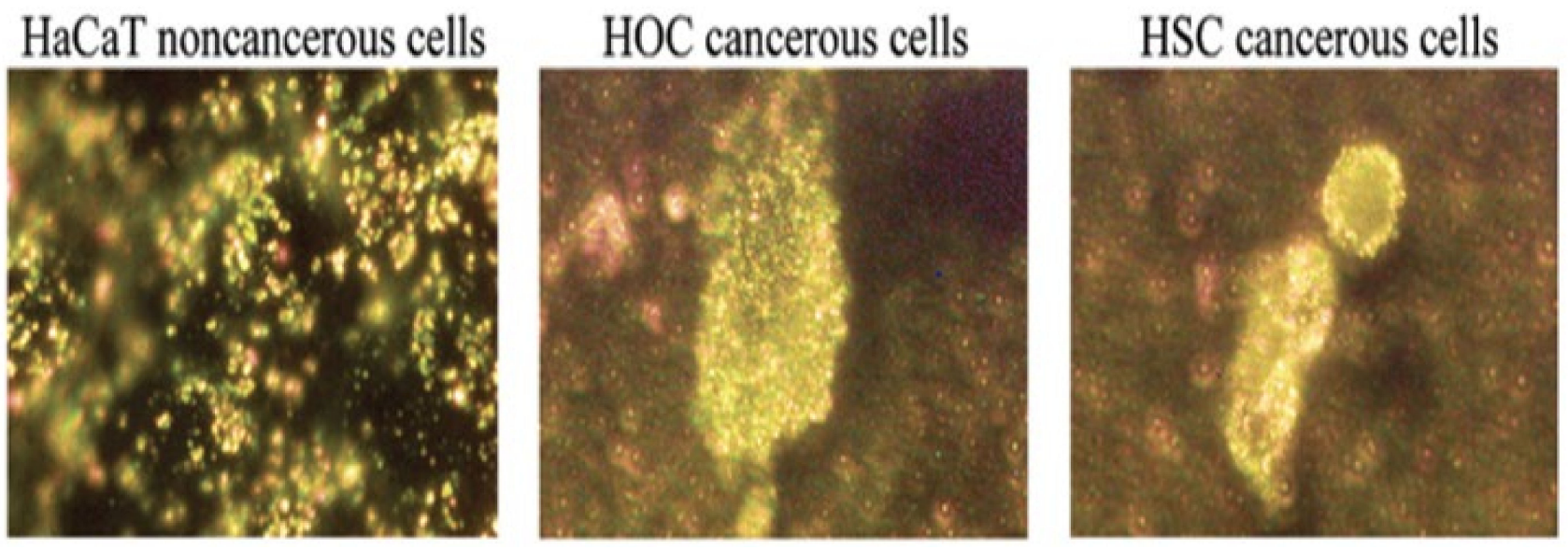

Figure 60. SPR light scattering images to distinguish between normal cells (left panel, HaCaT) and cancerous cells (middle and right panel, HOC and HSC) after incubation with anti-EGFR conjugated Au NPs. The anti-EGFR conjugated Au NPs bind specifically to the surface of cancer cells resulting in a sharper SPR band with a red-shifted maxima. Reproduced with permission from Nano Letter [500]. Copyright 2005, American Chemical Society.

\section{Conclusions and Outlook}

Due to the diverse nature and better stability against photo-corrosion and high redox ability of Au-based material, it has many potential applications in catalysis, oil hydro refining, drug carrier and electrode materials, solar cells, organic synthesis, water and air purification, cancer therapy, cathodic corrosion protection and self-cleaning antibacterial materials. It is concluded that such potential applications can further be enhanced by applying various new stratigies for the modification and hybridization of Au nanostructes with various organic, biochemical and inorganic materials, ranging from conventional molecules to polymeric macromolecules. A detailed understanding of the correlations between its modified structures/natures and its catalytic activity in a particular reaction still needs to be addressed. A combination of theoretical modelling and experimental modification procedures can open new windows in the advancement of Au-based nanostructures. This may be helpful both from academic and applied point of views. 
Author Contributions: Conceptualization, M.Y., M.H., A.K. and H.U. (Habib Ullah ${ }^{5}$ ); validation, M.H., A.K., A.A.T., H.U. (Habib Ullah ${ }^{5}$ ), M.U. and H.U. (Habib Ullah ${ }^{4}$ ); writing-original draft preparation, M.Y.; writing-review and editing, M.H., A.K. and H.U. (Habib Ullah ${ }^{5}$ ); visualization, M.H., A.K., A.A.T., H.U. (Habib Ullah ${ }^{4}$ ), M.U. and H.U. (Habib Ullah ${ }^{5}$ ); supervision, M.H., A.K. and H.U. (Habib Ullah ${ }^{5}$ ); All authors have read and agreed to the published version of the manuscript.

Funding: This research received no external funding.

Acknowledgments: We are thankful to the Engineering and Physical Science Research Council, UK (EPSRC under research grant no. EP/V049046/1 and EP/T025875/, and Saudi Aramco Chair Programme (ORCP2390) for financial support.

Conflicts of Interest: The authors declare no conflict of interest.

\section{References}

1. Wiesner, M.; Bottero, J.-Y. Environmental Nanotechnology; McGraw-Hill Professional Publishing New York: New York, NY, USA, 2007.

2. Di Guglielmo, C.; López, D.R.; De Lapuente, J.; Mallafre, J.M.L.; Suàrez, M.B. Embryotoxicity of cobalt ferrite and gold nanoparticles: A first in vitro approach. Reprod. Toxicol. 2010, 30, 271-276.

3. Eck, D.; Helm, C.A.; Wagner, N.J.; Vaynberg, K.A. Plasmon resonance measurements of the adsorption and adsorption kinetics of a biopolymer onto gold nanocolloids. Langmuir 2001, 17, 957-960.

4. Edwards, P.P.; Thomas, J.M. Gold in a metallic divided state-from faraday to present-day nanoscience. Angew. Chem. Int. Ed. 2007, 46, 5480-5486.

5. Kang, B.; Mackey, M.A.; El-Sayed, M.A. Nuclear targeting of gold nanoparticles in cancer cells induces DNA damage, causing cytokinesis arrest and apoptosis. J. Am. Chem. Soc. 2010, 132, 1517-1519.

6. Shang, C.; Liu, Z.-P. Origin and activity of gold nanoparticles as aerobic oxidation catalysts in aqueous solution. J. Am. Chem. Soc. 2011, 133, 9938-9947.

7. Abadeer, N.S.; Murphy, C.J. Recent progress in cancer thermal therapy using gold nanoparticles. J. Phys. Chem. C 2016, 120, 4691-4716.

8. Saleh, T.A. Spectroscopy: Between Modeling, Simulation and Practical Investigation. Spectr. Anal. Rev. 2014, 2, 1-2.

9. Saleh, T.A. Sensing of chlorpheniramine in pharmaceutical applications by sequential injector coupled with potentiometer. J. Pharm. Anal. 2011, 1, 246-250.

10. Kelly, K.L.; Coronado, E.; Zhao, L.L.; Schatz, G.C. The optical properties of metal nanoparticles: The influence of size, shape, and dielectric environment. J. Phys. Chem. B 2003, 107, 668-677.

11. Takami, A.; Kurita, H.; Koda, S. Laser-induced size reduction of noble metal particles. J. Phys. Chem. B 1999, 103, $1226-1232$.

12. Chen, D. Design, synthesis and properties of highly functional nanostructured photocatalysts. Recent Pat. Nanotechnol. 2008, 2, 183-189.

13. Balamurugan, B.; Maruyama, T. Evidence of an enhanced interband absorption in Au nanoparticles: Size-dependent electronic structure and optical properties. Appl. Phys. Lett. 2005, 87, 143105.

14. Chen, X.; Zheng, Z.; Ke, X.; Jaatinen, E.; Xie, T.; Wang, D.; Guo, C.; Zhao, J.; Zhu, H. Supported silver nanoparticles as photocatalysts under ultraviolet and visible light irradiation. Green Chem. 2010, 12, 414-419.

15. Litter, M.I. Heterogeneous photocatalysis: Transition metal ions in photocatalytic systems. Appl. Catal. B Environ. 1999, 23, 89-114.

16. Link, S.; El-Sayed, M. Steady state and time resolved optical properties of metallic nanoparticles the surface plasmon absorption as an analytical tool to inverstigate particle properties. Int. Rev. Phys. Chem. 2000, 19, 409.

17. Furube, A.; Du, L.; Hara, K.; Katoh, R.; Tachiya, M. Ultrafast plasmon-induced electron transfer from gold nanodots into TiO 2 nanoparticles. J. Am. Chem. Soc. 2007, 129, 14852-14853.

18. Grirrane, A.; Corma, A.; García, H. Gold-catalyzed synthesis of aromatic azo compounds from anilines and nitroaromatics. Science 2008, 322, 1661-1664.

19. Khumaeni, A.; Budi, W.S.; Sutanto, H. Synthesis and characterization of high-purity gold nanoparticles by laser ablation method using low-energy Nd: YAG laser 1064 nm. J. Phys. Conf. Ser. 2017, 909, 012037.

20. Eskandari-Nojedehi, M.; Jafarizadeh-Malmiri, H.; Rahbar-Shahrouzi, J. Hydrothermal green synthesis of gold nanoparticles using mushroom (Agaricus bisporus) extract: Physico-chemical characteristics and antifungal activity studies. Green Process. Synth. 2018, 7, 38-47.

21. Gutierrez-Wing, C.; Esparza, R.; Vargas-Hernandez, C.; Garcia, M.F.; Jose-Yacaman, M. Microwave-assisted synthesis of gold nanoparticles self-assembled into self-supported superstructures. Nanoscale 2012, 4, 2281-2287.

22. Sakai, T.; Enomoto, H.; Sakai, H.; Abe, M. Hydrogen-assisted fabrication of spherical gold nanoparticles through sonochemical reduction of tetrachloride gold (III) ions in water. Ultrason. Sonochem. 2014, 21, 946-950.

23. Hussain, R.K.; Abdulghani, A.J. Synthesis of gold nanoparticles via chemical reduction of Au (III) ions by isatin in aqueous solutions: Ligand concentrations and pH effects. Baghdad Sci. J. 2014, 11, 1201-1216.

24. Sadeghi, B.; Mohammadzadeh, M.; Babakhani, B. Green synthesis of gold nanoparticles using Stevia rebaudiana leaf extracts: Characterization and their stability. J. Photochem. Photobiol. B Biol. 2015, 148, 101-106. 
25. Zhu, L.; Zhang, C.; Guo, C.; Wang, X.; Sun, P.; Zhou, D.; Chen, W.; Xue, G. New insight into intermediate precursors of Brust-Schiffrin gold nanoparticles synthesis. J. Phys. Chem. C 2013, 117, 11399-11404.

26. Hoo, X.-F.; Razak, K.A.; Ridhuan, N.S.; Nor, N.M.; Zakaria, N.D. Synthesis of tunable size gold nanoparticles using seeding growth method and its application in glucose sensor. AIP Conf. Proc. 2017, 1877, 030001.

27. Ruivo, A.; Ventura, M.; da Silva, M.G.; Laia, C. Synthesis of gold nanoparticles in sol-gel glass porogens containing [bmim][BF 4] ionic liquid. J. Sol-Gel Sci. Technol. 2013, 68, 234-244.

28. Masanori Sakamoto, M.F.; Majima, T. Light as a construction tool of metal nanoparticles: Synthesis and mechanism. J. Photochem. Photobiol. C 2009, 10, 33-56.

29. Mian Rong Lee, H.K.L.; Yijie Yang, C.S.L.; Koh, C.L.L.; Lee, Y.H.; Phang, I.Y.; Ling, X.Y. Direct Metal Writing and Precise Positioning of Gold Nanoparticles within Microfluidic Channels for SERS Sensing of Gaseous Analytes. Acs Appl. Mater. Interfaces 2017, 9, 45 .

30. Izquierdo-Lorenzo, I.; Jradi, S.; Adam, P.-M. Direct laser writing of random Au nanoparticle three-dimensional structures for highly reproducible micro-SERS measurements. R. Soc. Chem. 2014, 4, 4128-4133.

31. Ritacco, T.; Pagliusi, P.; Giocondo, M. Insight into diffusive and convective processes affecting gold nanoparticles microclustering by multiphoton photoreduction. Colloids Surf. A Phys. Eng. Asp. 2021, 610, 125927.

32. Chen, Y.-S.; Hung, Y.-C.; Liau, I.; Huang, G.S. Assessment of the in vivo toxicity of gold nanoparticles. Nanoscale Res. Lett. 2009, 4, 858.

33. Colvin, V.L. The potential environmental impact of engineered nanomaterials. Nat. Biotechnol. 2003, 21, 1166-1170.

34. Shedbalkar, U.; Singh, R.; Wadhwani, S.; Gaidhani, S.; Chopade, B. Microbial synthesis of gold nanoparticles: Current status and future prospects. Adv. Colloid Interface Sci. 2014, 209, 40-48.

35. Thakkar, K.N.; Mhatre, S.S.; Parikh, R.Y. Biological synthesis of metallic nanoparticles. Nanomed. Nanotechnol. Biol. Med. 2010, 6, 257-262.

36. Dass, A.; Guo, R.; Tracy, J.B.; Balasubramanian, R.; Douglas, A.D.; Murray, R.W. Gold nanoparticles with perfluorothiolate ligands. Langmuir 2008, 24, 310-315.

37. Kroto, H.; Heath, J.O.; Brien, S.C.; Curl, R.F.; Smalley, R.E. C60 Buckminsterfullerene. Nature 1985, 318, 162-163.

38. Nakamura, E.; Isobe, H. Functionalized fullerenes in water. The first 10 years of their chemistry, biology, and nanoscience. Acc. Chem. Res. 2003, 36, 807-815.

39. Brust, M.; Kiely, C.J.; Bethell, D.; Schiffrin, D.J. C60 mediated aggregation of gold nanoparticles. J. Am. Chem. Soc. 1998, 120, 12367-12368.

40. Fujihara, H.; Nakai, H. Fullerenethiolate-functionalized gold nanoparticles: A new class of surface-confined metal-c60 nanocomposites. Langmuir 2001, 17, 6393-6395.

41. Sudeep, P.; Ipe, B.I.; Thomas, K.G.; George, M.; Barazzouk, S.; Hotchandani, S.; Kamat, P.V. Fullerene-functionalized gold nanoparticles. A self-assembled photoactive antenna-metal nanocore assembly. Nano Lett. 2002, 2, $29-35$.

42. Shon, Y.-S.; Choo, H. [60]Fullerene-linked gold nanoparticles: Synthesis and layer-by-layer growth on a solid surface. Chem. Commun. 2002, 2560-2561. [CrossRef]

43. Liu, J.; Alvarez, J.; Ong, W.; Kaifer, A.E. Network aggregates formed by C60 and gold nanoparticles capped with $\gamma$-cyclodextrin hosts. Nano Lett. 2001, 1, 57-60.

44. Hasobe, T.; Imahori, H.; Kamat, P.V.; Fukuzumi, S. Quaternary self-organization of porphyrin and fullerene units by clusterization with gold nanoparticles on $\mathrm{SnO}_{2}$ electrodes for organic solar cells. J. Am. Chem. Soc. 2003, 125, 14962-14963.

45. Islam, M.T.; Molugu, S.K.; Cooke, P.H.; Noveron, J.C. Fullerene stabilized gold nanoparticles. New J. Chem. 2015, $39,5923-5926$.

46. Frare, M.; Pilot, R.; De Filippo, C.; Weber, V.; Signorini, R.; Maggini, M.; Bozio, R. Fullerene functionalized gold nanoparticles for optical limiting of continuous wave lasers. Appl. Phys. B 2019, 125, 1-12.

47. Plesek, J. Potential applications of the boron cluster compounds. Chem. Rev. 1992, 92, 269-278.

48. Hawthorne, M.F.; Maderna, A. Applications of Radiolabeled Boron Clusters to the Diagnosis and Treatment of Cancer. Chem. Rev. 1999, 99, 3421-3434.

49. Barbera, G.; Vaca, A.; Teixidor, F.; Sillanpää, R.; Kivekäs, R.; Vinas, C. Designed synthesis of new ortho-carborane derivatives: From mono-to polysubstituted frameworks. Inorg. Chem. 2008, 47, 7309-7316.

50. Dash, B.P.; Satapathy, R.; Maguire, J.A.; Hosmane, N.S. Synthesis of a new class of carborane-containing star-shaped molecules via silicon tetrachloride promoted cyclotrimerization reactions. Org. Lett. 2008, 10, 2247-2250.

51. Smith, H., Jr.; Obenland, C.; Papetti, S. A New Series of Organoboranes. IX. The Preparation and Some Reactions of SulfurCarborane Derivatives. Inorg. Chem. 1966, 5, 1013-1015.

52. Plešek, J.; Heřmánek, S. Syntheses and properties of substituted icosahedral carborane thiols. Collect. Czechoslov. Chem. Commun. 1981, 46, 687-692.

53. Baše, T.; Bastl, Z.; Plzák, Z.; Grygar, T.; Plešek, J.; Carr, M.J.; Malina, V.; Šubrt, J.; Bohácek, J.; Večerníková, E. Carboranethiolmodified gold surfaces. A study and comparison of modified cluster and flat surfaces. Langmuir 2005, 21, 7776-7785.

54. Baše, T.S.; Bastl, Z.; Šlouf, M.; Klementová, M.; Šubrt, J.; Vetushka, A.; Ledinský, M.; Fejfar, A.; Machácek, J.; Carr, M.J. Gold micrometer crystals modified with carboranethiol derivatives. J. Phys. Chem. C 2008, 112, 14446-14455.

55. Grzelczak, M.P.; Danks, S.P.; Klipp, R.C.; Belic, D.; Zaulet, A.; Kunstmann-Olsen, C.; Bradley, D.F.; Tsukuda, T.; Viñas, C.; Teixidor, F. Ion transport across biological membranes by carborane-capped gold nanoparticles. Acs Nano 2017, 11, 12492-12499. 
56. Wang, J.; Chen, L.; Ye, J.; Li, Z.; Jiang, H.; Yan, H.; Stogniy, M.Y.; Sivaev, I.B.; Bregadze, V.I.; Wang, X. Carborane derivative conjugated with gold nanoclusters for targeted cancer cell imaging. Biomacromolecules 2017, 18, 1466-1472.

57. Bogireddy, N.; Pal, U.; Gomez, L.M.; Agarwal, V. Size controlled green synthesis of gold nanoparticles using Coffea arabica seed extract and their catalytic performance in 4-nitrophenol reduction. Rsc Adv. 2018, 8, 24819-24826.

58. Schmid, G.; Pugin, R.; Malm, J.O.; Bovin, J.O. Silsesquioxanes as ligands for gold clusters. Eur. J. Inorg. Chem. 1998, $1998,813-817$.

59. Carroll, J.B.; Frankamp, B.L.; Rotello, V.M. Self-assembly of gold nanoparticles through tandem hydrogen bonding and polyoligosilsequioxane (POSS)-POSS recognition processes. Chem. Commun. 2002, 1892-1893.

60. Carroll, J.B.; Frankamp, B.L.; Srivastava, S.; Rotello, V.M. Electrostatic self-assembly of structured gold nanoparticle/polyhedral oligomeric silsesquioxane (POSS) nanocomposites. J. Mater. Chem. 2004, 14, 690-694.

61. Zhang, Y.; Hao, J.; Xu, X.; Chen, X.; Wang, J. Protein Corona-Triggered Catalytic Inhibition of Insufficient POSS Polymer-Caged Gold Nanoparticles for Sensitive Colorimetric Detection of Metallothioneins. Anal. Chem. 2019, 92, $2080-2087$.

62. Xia, S.; Yang, Y.; Zhu, W.; Lü, C. Quaternized polyhedral oligomeric silsesquioxanes stabilized Pd nanoparticles as efficient nanocatalysts for reduction reaction. Colloids Surf. A Physicochem. Eng. Asp. 2020, 585, 124110.

63. Hanske, C.; Sanz-Ortiz, M.N.; Liz-Marzán, L.M. Silica-Coated Plasmonic Metal Nanoparticles in Action. Adv. Mater. 2018, 30, 1707003.

64. Chapman, B.S.; Wu, W.-C.; Li, Q.; Holten-Andersen, N.; Tracy, J.B. Heteroaggregation approach for depositing magnetite nanoparticles onto Silica-overcoated gold nanorods. Chem. Mater. 2017, 29, 10362-10368.

65. Liz-Marzán, L.M.; Giersig, M.; Mulvaney, P. Synthesis of nanosized gold-silica core-shell particles. Langmuir 1996, 12, 4329-4335.

66. Han, Y.; Jiang, J.; Lee, S.S.; Ying, J.Y. Reverse microemulsion-mediated synthesis of silica-coated gold and silver nanoparticles. Langmuir 2008, 24, 5842-5848.

67. Casavola, M.; Buonsanti, R.; Caputo, G.; Cozzoli, P.D. Colloidal strategies for preparing oxide-based hybrid nanocrystals. Eur. J. Inorg. Chem. 2008, 2008, 837-854.

68. Rodríguez-Fernández, J.; Pastoriza-Santos, I.; Perez-Juste, J.; García de Abajo, F.J.; Liz-Marzan, L.M. The effect of silica coating on the optical response of sub-micrometer gold spheres. J. Phys. Chem. C 2007, 111, 13361-13366.

69. Ruach-Nir, I.; Bendikov, T.A.; Doron-Mor, I.; Barkay, Z.; Vaskevich, A.; Rubinstein, I. Silica-stabilized gold island films for transmission localized surface plasmon sensing. J. Am. Chem. Soc. 2007, 129, 84-92.

70. Liu, G.; Ji, H.; Yang, X.; Wang, Y. Synthesis of a Au/silica/polymer trilayer composite and the corresponding hollow polymer microsphere with a movable Au core. Langmuir 2008, 24, 1019-1025.

71. Tovmachenko, O.G.; Graf, C.; van den Heuvel, D.J.; van Blaaderen, A.; Gerritsen, H.C. Fluorescence enhancement by metalcore/silica-shell nanoparticles. Adv. Mater. 2006, 18, 91-95.

72. Kamata, K.; Lu, Y.; Xia, Y. Synthesis and characterization of monodispersed core-shell spherical colloids with movable cores. J. Am. Chem. Soc. 2003, 125, 2384-2385.

73. Iijima, S. Synthesis of carbon nanotubes. Nature 1991, 354, 56-58.

74. Andrews, R.; Jacques, D.; Qian, D.; Rantell, T. Multiwall carbon nanotubes: Synthesis and application. Acc. Chem. Res. 2002, 35, 1008-1017.

75. Koo, W.-T.; Kim, Y.; Savagatrup, S.; Yoon, B.; Jeon, I.; Choi, S.-J.; Kim, I.-D.; Swager, T.M. Porous Ion Exchange Polymer Matrix for Ultrasmall Au Nanoparticle-Decorated Carbon Nanotube Chemiresistors. Chem. Mater. 2019, 31, 5413-5420.

76. Deshmukh, S.P.; Dhodamani, A.G.; Patil, S.M.; Mullani, S.B.; More, K.V.; Delekar, S.D. Interfacially Interactive Ternary SilverSupported Polyaniline/Multiwalled Carbon Nanotube Nanocomposites for Catalytic and Antibacterial Activity. Acs Omega 2019, $5,219-227$.

77. LeeáTan, K. Growth of Pd, Pt, Ag and Au nanoparticles on carbon nanotubes. J. Mater. Chem. 2001, 11, $2378-2381$.

78. Han, L.; Wu, W.; Kirk, F.L.; Luo, J.; Maye, M.M.; Kariuki, N.N.; Lin, Y.; Wang, C.; Zhong, C.-J. A direct route toward assembly of nanoparticle-carbon nanotube composite materials. Langmuir 2004, 20, 6019-6025.

79. Sainsbury, T.; Ikuno, T.; Okawa, D.; Pacile, D.; Frechet, J.M.; Zettl, A. Self-assembly of gold nanoparticles at the surface of amine-and thiol-functionalized boron nitride nanotubes. J. Phys. Chem. C 2007, 111, 12992-12999.

80. Jiang, K.; Eitan, A.; Schadler, L.S.; Ajayan, P.M.; Siegel, R.W.; Grobert, N.; Mayne, M.; Reyes-Reyes, M.; Terrones, H.; Terrones, M. Selective attachment of gold nanoparticles to nitrogen-doped carbon nanotubes. Nano Lett. 2003, 3, $275-277$.

81. Correa-Duarte, M.A.; Liz-Marzán, L.M. Carbon nanotubes as templates for one-dimensional nanoparticle assemblies. J. Mater. Chem. 2006, 16, 22-25.

82. Fu, Q.; Lu, C.; Liu, J. Selective coating of single wall carbon nanotubes with thin $\mathrm{SiO}_{2}$ layer. Nano Lett. $2002,2,329-332$.

83. Bottini, M.; Magrini, A.; Rosato, N.; Bergamaschi, A.; Mustelin, T. Dispersion of pristine single-walled carbon nanotubes in water by a thiolated organosilane: Application in supramolecular nanoassemblies. J. Phys. Chem. B 2006, 110, 13685-13688.

84. Ou, Y.-Y.; Huang, M.H. High-density assembly of gold nanoparticles on multiwalled carbon nanotubes using 1-pyrenemethylamine as interlinker. J. Phys. Chem. B 2006, 110, 2031-2036.

85. Hill, C.L. Introduction: Polyoxometalates multicomponent molecular vehicles to probe fundamental issues and practical problems. Chem. Rev. 1998, 98, 1-2.

86. Wang, Y.; Neyman, A.; Arkhangelsky, E.; Gitis, V.; Meshi, L.; Weinstock, I.A. Self-assembly and structure of directly imaged inorganic-anion monolayers on a gold nanoparticle. J. Am. Chem. Soc. 2009, 131, 17412-17422. 
87. Wang, Y.; Zeiri, O.; Sharet, S.; Weinstock, I.A. Role of the alkali-metal cation size in the self-assembly of polyoxometalatemonolayer shells on gold nanoparticles. Inorg. Chem. 2012, 51, 7436-7438.

88. Sutter, S.; Trepka, B.; Siroky, S.; Hagedorn, K.; Theiß, S.; Baum, P.; Polarz, S. Light-Triggered Boost of Activity of Catalytic Bola-Type Surfactants by a Plasmonic Metal-Support Interaction Effect. Acs Appl. Mater. Interfaces 2019, 11, 15936-15944.

89. Solarska, R.; Bienkowski, K.; Zoladek, S.; Majcher, A.; Stefaniuk, T.; Kulesza, P.J.; Augustynski, J. Enhanced water splitting at thin film tungsten trioxide photoanodes bearing plasmonic gold-polyoxometalate particles. Angew. Chem. Int. Ed. 2014, 53, 14196-14200.

90. Dolbecq, A.; Dumas, E.; Mayer, C.R.; Mialane, P. Hybrid organic-inorganic polyoxometalate compounds: From structural diversity to applications. Chem. Rev. 2010, 110, 6009-6048.

91. Mayer, C.R.; Neveu, S.; Cabuil, V. A nanoscale hybrid system based on gold nanoparticles and heteropolyanions. Angew. Chem. Int. Ed. 2002, 41, 501-503.

92. Hegde, S.; Joshi, S.; Mukherjee, T.; Kapoor, S. Formation of gold nanoparticles via a thiol functionalized polyoxometalate. Mater. Sci. Eng. C 2013, 33, 2332-2337.

93. Martín, S.; Takashima, Y.; Lin, C.-G.; Song, Y.-F.; Miras, H.N.; Cronin, L. Integrated synthesis of gold nanoparticles coated with polyoxometalate clusters. Inorg. Chem. 2019, 58, 4110-4116.

94. Tomane, S.; López-Maya, E.; Boujday, S.; Humblot, V.; Marrot, J.; Rabasso, N.; Castells-Gil, J.; Sicard, C.; Dolbecq, A.; Mialane, P. Correction: One-pot synthesis of a new generation of hybrid bisphosphonate polyoxometalate gold nanoparticles as antibiofilm agents. Nanoscale Adv. 2019, 1, 4173.

95. Wilton-Ely, J.D. The surface functionalisation of gold nanoparticles with metal complexes. Dalton Trans. 2008, 25-29. [CrossRef]

96. Stiles, R.L.; Balasubramanian, R.; Feldberg, S.W.; Murray, R.W. Anion-induced adsorption of ferrocenated nanoparticles. J. Am. Chem. Soc. 2008, 130, 1856-1865.

97. Wolfe, R.L.; Balasubramanian, R.; Tracy, J.B.; Murray, R.W. Fully ferrocenated hexanethiolate monolayer-protected gold clusters. Langmuir 2007, 23, 2247-2254.

98. Chen, S.; Murray, R.W. Arenethiolate monolayer-protected gold clusters. Langmuir 1999, 15, 682-689.

99. Li, D.; Zhang, Y.; Jiang, J.; Li, J. Electroactive gold nanoparticles protected by 4-ferrocene thiophenol monolayer. J. Colloid Interface Sci. 2003, 264, 109-113.

100. Li, D.; Zhang, Y.; Li, J. Electrochemical study of 4-ferrocene thiophenol monolayers assembled on gold nanoparticles. Microelectron. Eng. 2003, 66, 91-94.

101. Ingram, R.S.; Hostetler, M.J.; Murray, R.W. Poly-hetero- $\omega$-functionalized alkanethiolate-stabilized gold cluster compounds. J. Am. Chem. Soc. 1997, 119, 9175-9178.

102. Green, S.J.; Stokes, J.J.; Hostetler, M.J.; Pietron, J.; Murray, R.W. Three-dimensional monolayers: Nanometer-sized electrodes of alkanethiolate-stabilized gold cluster molecules. J. Phys. Chem. B 1997, 101, 2663-2668.

103. Ornelas, C.; Méry, D.; Cloutet, E.; Aranzaes, J.R.; Astruc, D. Cross olefin metathesis for the selective functionalization, ferrocenylation, and solubilization in water of olefin-terminated dendrimers, polymers, and gold nanoparticles and for a divergent dendrimer construction. J. Am. Chem. Soc. 2008, 130, 1495-1506.

104. Labande, A.; Astruc, D. Colloids as redox sensors: Recognition of $\mathrm{H}_{2} \mathrm{PO}^{4-}$ and $\mathrm{HSO}^{4-}$ by amidoferrocenylalkylthiol-gold nanoparticles. Chem. Commun. 2000, 124, 1007-1008.

105. Wang, Y.; Salmon, L.; Ruiz, J.; Astruc, D. Metallodendrimers in three oxidation states with electronically interacting metals and stabilization of size-selected gold nanoparticles. Nat. Commun. 2014, 5, 1-12.

106. Labande, A.; Ruiz, J.; Astruc, D. Supramolecular gold nanoparticles for the redox recognition of oxoanions: Syntheses, titrations, stereoelectronic effects, and selectivity. J. Am. Chem. Soc. 2002, 124, 1782-1789.

107. Horikoshi, T.; Itoh, M.; Kurihara, M.; Kubo, K.; Nishihara, H. Synthesis, redox behavior and electrodeposition of biferrocenemodified gold clusters. J. Electroanal. Chem. 1999, 473, 113-116.

108. Yamada, M.; Tadera, T.; Kubo, K.; Nishihara, H. Electrochemical deposition of biferrocene derivative-attached gold nanoparticles and the morphology of the formed film. J. Phys. Chem. B 2003, 107, 3703-3711.

109. Dong, T.-Y.; Shih, H.-W.; Chang, L.-S. Synthesis and redox behavior of biferrocenyl-functionalized ruthenium (II) terpyridine gold clusters. Langmuir 2004, 20, 9340-9347.

110. Daniel, M.-C.; Ruiz, J.; Nlate, S.; Palumbo, J.; Blais, J.-C.; Astruc, D. Gold nanoparticles containing redox-active supramolecular dendrons that recognize H2PO4- ${ }^{-}$. Chem. Commun. 2001, 2000-2001.

111. Daniel, M.-C.; Ruiz, J.; Nlate, S.; Blais, J.-C.; Astruc, D. Nanoscopic assemblies between supramolecular redox active metallodendrons and gold nanoparticles: Synthesis, characterization, and selective recognition of $\mathrm{H} 2 \mathrm{PO}^{4-}$, $\mathrm{HSO}^{4-}$, and adenosine- $5^{\prime}-$ triphosphate (ATP2-) anions. J. Am. Chem. Soc. 2003, 125, 2617-2628.

112. Astruc, D.; Daniel, M.-C.; Ruiz, J. Dendrimers and gold nanoparticles as exo-receptors sensing biologically important anions. Chem. Commun. 2004, 2637-2649.

113. Liu, F.; Liu, X.; Astruc, D.; Gu, H. Dendronized triazolyl-containing ferrocenyl polymers as stabilizers of gold nanoparticles for recyclable two-phase reduction of 4-nitrophenol. J. Colloid Interface Sci. 2019, 533, 161-170.

114. Opuchlik, L.J.; Pawłowska, J.; Sęk, S.; Bilewicz, R. Ferrocenylated gold nanoparticles self-assemble at carbon surfaces to form stable films. J. Electroanal. Chem. 2018, 825, 22-29. 
115. Vitale, F.; Vitaliano, R.; Battocchio, C.; Fratoddi, I.; Piscopiello, E.; Tapfer, L.; Russo, M.V. Synthesis and characterization of gold nanoparticles stabilized by palladium (II) phosphine thiol. J. Organomet. Chem. 2008, 693, 1043-1048.

116. Bartz, M.; Küther, J.; Seshadri, R.; Tremel, W. Colloid-Bound Catalysts for Ring-Opening Metathesis Polymerization: A Combination of Homogenous and Heterogeneous Properties. Angew. Chem. Int. Ed. 1998, 37, 2466-2468.

117. Wang, S.; Sim, W.-S. Au nanoparticles encapsulated in Ru carbonyl carboxylate shells. Langmuir 2006, 22, 7861-7866.

118. Belser, T.; Stöhr, M.; Pfaltz, A. Immobilization of rhodium complexes at thiolate monolayers on gold surfaces: Catalytic and structural studies. J. Am. Chem. Soc. 2005, 127, 8720-8731.

119. Kumar, S.S.; Joseph, J.; Phani, K.L. Novel method for deposition of Gold-Prussian blue nanocomposite films induced by electrochemically formed gold nanoparticles: Characterization and application to electrocatalysis. Chem. Mater. 2007, 19, $4722-4730$.

120. Crespilho, F.N.; Zucolotto, V.; Brett, C.M.; Oliveira, O.N.; Nart, F.C. Enhanced charge transport and incorporation of redox mediators in layer-by-layer films containing PAMAM-encapsulated gold nanoparticles. J. Phys. Chem. B 2006, 110, 17478-17483.

121. Qiu, J.-D.; Peng, H.-Z.; Liang, R.-P.; Li, J.; Xia, X.-H. Synthesis, characterization, and immobilization of Prussian blue-modified Au nanoparticles: Application to electrocatalytic reduction of $\mathrm{H}_{2} \mathrm{O}_{2}$. Langmuir 2007, 23, 2133-2137.

122. Dumur, F.; Dumas, E.; Mayer, C.R. Functionalization of Gold Nanoparticles by Inorganic Entities. Nanomaterials 2020, $10,548$.

123. Toma, S.H.; Bonacin, J.A.; Araki, K.; Toma, H.E. Controlled Stabilization and Flocculation of Gold Nanoparticles by Means of 2-Pyrazin-2-ylethanethiol and Pentacyanidoferrate (II) Complexes. Eur. J. Inorg. Chem. 2007, 2007, 3356-3364.

124. Nunes, F.S.; Bonifácio, L.D.S.; Araki, K.; Toma, H.E. Interaction of 2-and 4-mercaptopyridine with pentacyanoferrates and gold nanoparticles. Inorg. Chem. 2006, 45, 94-101.

125. Cheng, P.P.H.; Silvester, D.; Wang, G.; Kalyuzhny, G.; Douglas, A.; Murray, R.W. Dynamic and static quenching of fluorescence by 1-4 nm diameter gold monolayer-protected clusters. J. Phys. Chem. B 2006, 110, 4637-4644.

126. Brewer, S.H.; Glomm, W.R.; Johnson, M.C.; Knag, M.K.; Franzen, S. Probing BSA binding to citrate-coated gold nanoparticles and surfaces. Langmuir 2005, 21, 9303-9307.

127. Xu, X.-H.N.; Huang, S.; Brownlow, W.; Salaita, K.; Jeffers, R.B. Size and temperature dependence of surface plasmon absorption of gold nanoparticles induced by tris (2, 2'-bipyridine) ruthenium (II). J. Phys. Chem. B 2004, 108, 15543-15551.

128. Pramod, P.; Sudeep, P.; Thomas, K.G.; Kamat, P.V. Photochemistry of ruthenium trisbipyridine functionalized on gold nanoparticles. J. Phys. Chem. B 2006, 110, 20737-20741.

129. Jebb, M.; Sudeep, P.; Pramod, P.; Thomas, K.G.; Kamat, P.V. Ruthenium (II) trisbipyridine functionalized gold nanorods. Morphological changes and excited-state interactions. J. Phys. Chem. B 2007, 111, 6839-6844.

130. Sun, X.; Du, Y.; Dong, S.; Wang, E. Method for effective immobilization of Ru (bpy) $3^{2+}$ on an electrode surface for solid-state electrochemiluminescene detection. Anal. Chem. 2005, 77, 8166-8169.

131. Liu, J.; Lu, Y. Stimuli-responsive disassembly of nanoparticle aggregates for light-up colorimetric sensing. J. Am. Chem. Soc. 2005, $127,12677-12683$.

132. Kuwahara, Y.; Akiyama, T.; Yamada, S. Construction of gold nanoparticle-ruthenium (II) tris (2, 2'-bipyridine) self-assembled multistructures and their photocurrent responses. Thin Solid Film. 2001, 393, 273-277.

133. Akiyama, T.; Inoue, K.; Kuwahara, Y.; Terasaki, N.; Niidome, Y.; Yamada, S. Particle-size effects on the photocurrent efficiency of nanostructured assemblies consisting of gold nanoparticles and a ruthenium complex-viologen linked thiol. J. Electroanal. Chem. 2003, 550, 303-307.

134. Akiyama, T.; Inoue, K.; Kuwahara, Y.; Niidome, Y.; Terasaki, N.; Nitahara, S.; Yamada, S. Facile fabrication of morphologycontrolled gold nanoparticle architectures by electrolyte-induced agglomeration and their photoelectrochemical applications. Langmuir 2005, 21, 793-796.

135. Lahav, M.; Heleg-Shabtai, V.; Wasserman, J.; Katz, E.; Willner, I.; Dürr, H.; Hu, Y.-Z.; Bossmann, S.H. Photoelectrochemistry with Integrated Photosensitizer-Electron Acceptor and Au-Nanoparticle Arrays. J. Am. Chem. Soc. 2000, 122, $11480-11487$.

136. Shipway, A.N.; Lahav, M.; Willner, I. Nanostructured gold colloid electrodes. Adv. Mater. 2000, 12, $993-998$.

137. Chen, S.; Pei, R.; Zhao, T.; Dyer, D.J. Gold Nanoparticle Assemblies by Metal Ion-Pyridine Complexation and Their Rectified Quantized Charging in Aqueous Solutions. J. Phys. Chem. B 2002, 106, 1903-1908.

138. Soller, T.; Ringler, M.; Wunderlich, M.; Klar, T.; Feldmann, J.; Josel, H.-P.; Markert, Y.; Nichtl, A.; Kürzinger, K. Radiative and nonradiative rates of phosphors attached to gold nanoparticles. Nano Lett. 2007, 7, 1941-1946.

139. Slim, M.; Durisic, N.; Grutter, P.; Sleiman, H.F. DNA-Protein Noncovalent Cross-Linking: Ruthenium Dipyridophenazine Biotin Complex for the Assembly of Proteins and Gold Nanoparticles on DNA Templates. ChemBioChem 2007, 8, 804-812.

140. Shultz, D.A.; Kumar, R.K.; Bin-Salamon, S.; Kirk, M.L. Valence tautomerization and exchange coupling in a cobaltnitronylnitroxide-semiquinone complex. Polyhedron 2005, 24, 2876-2879.

141. Barmin, R.A.; Rudakovskaya, P.G.; Gusliakova, O.I.; Sindeeva, O.A.; Prikhozhdenko, E.S.; Maksimova, E.A.; Obukhova, E.N.; Chernyshev, V.S.; Khlebtsov, B.N.; Solovev, A.A.; et al. Air-Filled Bubbles Stabilized by Gold Nanoparticle/Photodynamic Dye Hybrid Structures for Theranostics. Nanomaterials 2021, 11, 415.

142. Mayer, C.R.; Dumas, E.; Miomandre, F.; Méallet-Renault, R.; Warmont, F.; Vigneron, J.; Pansu, R.; Etcheberry, A.; Sécheresse, F. Polypyridyl ruthenium complexes as coating agent for the formation of gold and silver nanocomposites in different media. Preliminary luminescence and electrochemical studies. New J. Chem. 2006, 30, 1628-1637. 
143. Pérez León, C.; Kador, L.; Peng, B.; Thelakkat, M. Influence of the solvent on the surface-enhanced Raman spectra of ruthenium (II) bipyridyl complexes. J. Phys. Chem. B 2005, 109, 5783-5789.

144. Huang, W.; Tanaka, H.; Ogawa, T. Effects of Metal-Ion Complexation for the Self-Assembled Nanocomposite Films Composed of Gold Nanoparticles and 3, 8-Bis (terthiophenyl) phenanthroline-Based Dithiols Bridging $1 \mu \mathrm{m}$ Gap Gold Electrodes: Morphology, Temperature Dependent Electronic Conduction, and Photoresponse. J. Phys. Chem. C 2008, 112, 11513-11526.

145. Ono, F.; Kanemasa, S.; Tanaka, J. Reusable nano-sized chiral bisoxazoline catalysts. Tetrahedron Lett. 2005, 46, 7623-7626.

146. Hone, D.C.; Walker, P.I.; Evans-Gowing, R.; FitzGerald, S.; Beeby, A.; Chambrier, I.; Cook, M.J.; Russell, D.A. Generation of cytotoxic singlet oxygen via phthalocyanine-stabilized gold nanoparticles: A potential delivery vehicle for photodynamic therapy. Langmuir 2002, 18, 2985-2987.

147. Beer, P.D.; Cormode, D.P.; Davis, J.J. Zinc metalloporphyrin-functionalised nanoparticle anion sensors. Chem. Commun. 2004, 414-415.

148. Ozawa, H.; Kawao, M.; Tanaka, H.; Ogawa, T. Synthesis of dendron-protected porphyrin wires and preparation of a onedimensional assembly of gold nanoparticles chemically linked to the $\pi$-conjugated wires. Langmuir 2007, $23,6365-6371$.

149. Aubin-Tam, M.-E.; Hamad-Schifferli, K. Gold nanoparticle-cytochrome C complexes: The effect of nanoparticle ligand charge on protein structure. Langmuir 2005, 21, 12080-12084.

150. Sheet, D.; Bera, A.; Jana, R.D.; Paine, T.K. Oxidizing Ability of a Dioxygen-Activating Nonheme Iron (II)-Benzilate Complex Immobilized on Gold Nanoparticles. Inorg. Chem. 2019, 58, 4828-4841.

151. Huang, C.C.; Yang, Z.; Lee, K.H.; Chang, H.T. Synthesis of highly fluorescent gold nanoparticles for sensing mercury (II). Angew. Chem. 2007, 119, 6948-6952.

152. Huang, C.-C.; Chang, H.-T. Parameters for selective colorimetric sensing of mercury (II) in aqueous solutions using mercaptopropionic acid-modified gold nanoparticles. Chem. Commun. 2007, 1215-1217.

153. Zamborini, F.P.; Hicks, J.F.; Murray, R.W. Quantized double layer charging of nanoparticle films assembled using carboxylate/ $\left(\mathrm{Cu}^{2+}\right.$ or $\left.\mathrm{Zn}^{2+}\right) /$ carboxylate bridges. J. Am. Chem. Soc. 2000, 122, 4514-4515.

154. Zamborini, F.P.; Leopold, M.C.; Hicks, J.F.; Kulesza, P.J.; Malik, M.A.; Murray, R.W. Electron hopping conductivity and vapor sensing properties of flexible network polymer films of metal nanoparticles. J. Am. Chem. Soc. 2002, 124, 8958-8964.

155. Hicks, J.F.; Zamborini, F.P.; Murray, R.W. Dynamics of electron transfers between electrodes and monolayers of nanoparticles. J. Phys. Chem. B 2002, 106, 7751-7757.

156. Sheibley, D.; Tognarelli, D.; Szymanik, R.; Leopold, M.C. Ultra-fast formation and characterization of stable nanoparticle film assemblies. J. Mater. Chem. 2005, 15, 491-498.

157. Xu, R.; Wang, D.; Zhang, J.; Li, Y. Shape-dependent catalytic activity of silver nanoparticles for the oxidation of styrene. Chem. Asian J. 2006, 1, 888-893.

158. Wanunu, M.; Popovitz-Biro, R.; Cohen, H.; Vaskevich, A.; Rubinstein, I. Coordination-based gold nanoparticle layers. J. Am. Chem. Soc. 2005, 127, 9207-9215.

159. Mayer, C.R.; Cucchiaro, G.; Jullien, J.; Dumur, F.; Marrot, J.; Dumas, E.; Sécheresse, F. Functionalization of gold nanoparticles by iron (III) complexes derived from Schiff base ligands. Eur. J. Inorg. Chem. 2008, 2008, 3614-3623.

160. Guo, C.; Boullanger, P.; Jiang, L.; Liu, T. Highly sensitive gold nanoparticles biosensor chips modified with a self-assembled bilayer for detection of Con A. Biosens. Bioelectron. 2007, 22, 1830-1834.

161. Türkekul, K.; Üzer, A.; Can, Z.; Erçă̆, E.; Apak, R. Colorimetric Sensing of the Insensitive Energetic Material 3-Nitro-1, 2, 4-triazol-5-one (NTO) Using 1-Cysteine Stabilized Gold Nanoparticles and Copper (II). Anal. Lett. 2019, 52, $2809-2821$.

162. Abad, J.M.; Mertens, S.F.; Pita, M.; Fernández, V.M.; Schiffrin, D.J. Functionalization of thioctic acid-capped gold nanoparticles for specific immobilization of histidine-tagged proteins. J. Am. Chem. Soc. 2005, 127, 5689-5694.

163. Reynolds, A.J.; Haines, A.H.; Russell, D.A. Gold glyconanoparticles for mimics and measurement of metal ion-mediated carbohydrate-carbohydrate interactions. Langmuir 2006, 22, 1156-1163.

164. Si, S.; Kotal, A.; Mandal, T.K. One-dimensional assembly of peptide-functionalized gold nanoparticles: An approach toward mercury ion sensing. J. Phys. Chem. C 2007, 111, 1248-1255.

165. Lee, J.S.; Han, M.S.; Mirkin, C.A. Colorimetric detection of mercuric ion $\left(\mathrm{Hg}^{2+}\right)$ in aqueous media using DNA-functionalized gold nanoparticles. Angew. Chem. Int. Ed. 2007, 46, 4093-4096.

166. Liu, J.; Lu, Y. A colorimetric lead biosensor using DNAzyme-directed assembly of gold nanoparticles. J. Am. Chem. Soc. 2003, 125, 6642-6643.

167. Liu, J.; Lu, Y. Accelerated color change of gold nanoparticles assembled by DNAzymes for simple and fast colorimetric $\mathrm{Pb}^{2+}$ detection. J. Am. Chem. Soc. 2004, 126, 12298-12305.

168. Liu, J.; Lu, Y. Optimization of a $\mathrm{Pb}^{2+}$-directed gold nanoparticle/DNAzyme assembly and its application as a colorimetric biosensor for $\mathrm{Pb}^{2+}$. Chem. Mater. 2004, 16, 3231-3238.

169. Liu, J.; Lu, Y. Colorimetric $\mathrm{Cu}^{2+}$ detection with a ligation DNAzyme and nanoparticles. Chem. Commun. 2007, 4872-4874. [CrossRef]

170. Marubayashi, K.; Takizawa, S.; Kawakusu, T.; Arai, T.; Sasai, H. Monolayer-Protected Au Cluster (MPC)-Supported Ti- BINOLate Complex. Org. Lett. 2003, 5, 4409-4412.

171. Rogers, N.J.; Claire, S.; Harris, R.M.; Farabi, S.; Zikeli, G.; Styles, I.B.; Hodges, N.J.; Pikramenou, Z. High coating of Ru (II) complexes on gold nanoparticles for single particle luminescence imaging in cells. Chem. Commun. 2014, 50, 617-619. 
172. Thangavel, N.; Jayakumar, I.; Ravichandran, M.; Ganesan, V.V.; Nair, B.U. Photocrosslinking of collagen using Ru (II)-polypyridyl complex functionalized gold nanoparticles. Spectrochim. Acta Part A Mol. Biomol. Spectrosc. 2019, 215, $196-202$.

173. Cox, J.A.; Kittredge, K.W.; Ca, D.V. Measurement platforms fabricated by layer-by-layer assembly of crown ether functionalized gold nanoclusters. J. Solid State Electrochem. 2004, 8, 722-726.

174. Lin, S.Y.; Wu, S.H.; Chen, C.h. A simple strategy for prompt visual sensing by gold nanoparticles: General applications of interparticle hydrogen bonds. Angew. Chem. 2006, 118, 5070-5073.

175. Wang, C.; Yu, C. Detection of chemical pollutants in water using gold nanoparticles as sensors: A review. Rev. Anal. Chem. 2013, 32, 1-14.

176. Yamauchi, A.; Hayashita, T.; Nishizawa, S.; Watanabe, M.; Teramae, N. Benzo-15-crown-5 fluoroionophore/ $\gamma$-cyclodextrin complex with remarkably high potassium ion sensitivity and selectivity in water. J. Am. Chem. Soc. 1999, 121, $2319-2320$.

177. Xia, W.-S.; Schmehl, R.H.; Li, C.-J. A highly selective fluorescent chemosensor for $\mathrm{K}^{+}$from a bis-15-crown-5 derivative. J. Am. Chem. Soc. 1999, 121, 5599-5600.

178. Siddique, S.; Chow, J.C.L. Gold Nanoparticles for Drug Delivery and Cancer Therapy. Appl. Sci. 2020, $10,3824$.

179. Lin, S.-Y.; Liu, S.-W.; Lin, C.-M.; Chen, C.-h. Recognition of potassium ion in water by 15 -crown-5 functionalized gold nanoparticles. Anal. Chem. 2002, 74, 330-335.

180. Pompano, R.R.; Wortley, P.G.; Moatz, L.M.; Tognarelli, D.; Kittredge, K.W.; Leopold, M.C. Crown ether-metal "sandwiches" as linking mechanisms in assembled nanoparticle films. Thin Solid Film. 2006, 510, 311-319.

181. Lin, S.-Y.; Chen, C.-h.; Lin, M.-C.; Hsu, H.-F. A cooperative effect of bifunctionalized nanoparticles on recognition: Sensing alkali ions by crown and carboxylate moieties in aqueous media. Anal. Chem. 2005, 77, 4821-4828.

182. Gao, J.; Fu, J.; Lin, C.; Lin, J.; Han, Y.; Yu, X.; Pan, C. Formation and photoluminescence of silver nanoparticles stabilized by a two-armed polymer with a crown ether core. Langmuir 2004, 20, 9775-9779.

183. Kuang, H.; Chen, W.; Yan, W.; Xu, L.; Zhu, Y.; Liu, L.; Chu, H.; Peng, C.; Wang, L.; Kotov, N.A. Crown ether assembly of gold nanoparticles: Melamine sensor. Biosens. Bioelectron. 2011, 26, 2032-2037.

184. Sung, S.; Holmes, H.; Wainwright, L.; Toscani, A.; Stasiuk, G.J.; White, A.J.; Bell, J.D.; Wilton-Ely, J.D. Multimetallic complexes and functionalized gold nanoparticles based on a combination of d-and f-elements. Inorg. Chem. 2014, 53, $1989-2005$.

185. Truman, L.K.; Bradberry, S.J.; Comby, S.; Kotova, O.; Gunnlaugsson, T. Surface-Modified Gold Nanoparticles Possessing Two-Channel Responsive EuIII/TbIII Cyclen Complexes as Luminescent Logic Gate Mimics. ChemPhysChem 2017, 18, 1746-1751.

186. Lewis, D.J.; Day, T.M.; MacPherson, J.V.; Pikramenou, Z. Luminescent nanobeads: Attachment of surface reactive Eu (III) complexes to gold nanoparticles. Chem. Commun. 2006, 1433-1435. [CrossRef]

187. Massue, J.; Quinn, S.J.; Gunnlaugsson, T. Lanthanide luminescent displacement assays: The sensing of phosphate anions using $\mathrm{Eu}$ (III)-Cyclen-conjugated gold nanoparticles in aqueous solution. J. Am. Chem. Soc. 2008, 130, 6900-6901.

188. Ipe, B.I.; Yoosaf, K.; Thomas, K.G. Functionalized gold nanoparticles as phosphorescent nanomaterials and sensors. J. Am. Chem. Soc. 2006, 128, 1907-1913.

189. Alric, C.; Taleb, J.; Le Duc, G.; Mandon, C.; Billotey, C.; Le Meur-Herland, A.; Brochard, T.; Vocanson, F.; Janier, M.; Perriat, P. Gadolinium chelate coated gold nanoparticles as contrast agents for both X-ray computed tomography and magnetic resonance imaging. J. Am. Chem. Soc. 2008, 130, 5908-5915.

190. Debouttière, P.J.; Roux, S.; Vocanson, F.; Billotey, C.; Beuf, O.; Favre-Réguillon, A.; Lin, Y.; Pellet-Rostaing, S.; Lamartine, R.; Perriat, P. Design of gold nanoparticles for magnetic resonance imaging. Adv. Funct. Mater. 2006, 16, $2330-2339$.

191. Moriggi, L.; Cannizzo, C.; Dumas, E.; Mayer, C.R.; Ulianov, A.; Helm, L. Gold nanoparticles functionalized with gadolinium chelates as high-relaxivity MRI contrast agents. J. Am. Chem. Soc. 2009, 131, 10828-10829.

192. Liu, J.; Xiong, Z.; Zhang, J.; Peng, C.; Klajnert-Maculewicz, B.; Shen, M.; Shi, X. Zwitterionic gadolinium (III)-complexed dendrimer-entrapped gold nanoparticles for enhanced computed tomography/magnetic resonance imaging of lung cancer metastasis. Acs Appl. Mater. Interfaces 2019, 11, 15212-15221.

193. Martins, J.; Ferreira, P.M.; Geraldes, C.F.; Helm, L.; Prata, M.I.; Rodrigues, S.P.; Rodrigues, T.B.; Calle, D.; López-Larrubia, P.; Cerdan, S. Gold nanoparticles functionalised with fast water exchanging $\mathrm{Gd}^{3+}$ chelates: Linker effects on the relaxivity. Dalton Trans. 2015, 44, 4016-4031.

194. Haiss, W.; Thanh, N.T.; Aveyard, J.; Fernig, D.G. Determination of size and concentration of gold nanoparticles from UV-Vis spectra. Anal. Chem. 2007, 79, 4215-4221.

195. Perrault, S.D.; Chan, W.C. Synthesis and surface modification of highly monodispersed, spherical gold nanoparticles of 50-200 nm. J. Am. Chem. Soc. 2009, 131, 17042-17043.

196. Saha, K.; Agasti, S.S.; Kim, C.; Li, X.; Rotello, V.M. Gold nanoparticles in chemical and biological sensing. Chem. Rev. 2012, 112, 2739-2779.

197. Li, K.; Wang, K.; Qin, W.; Deng, S.; Li, D.; Shi, J.; Huang, Q.; Fan, C. DNA-directed assembly of gold nanohalo for quantitative plasmonic imaging of single-particle catalysis. J. Am. Chem. Soc. 2015, 137, 4292-4295.

198. Young, K.L.; Ross, M.B.; Blaber, M.G.; Rycenga, M.; Jones, M.R.; Zhang, C.; Senesi, A.J.; Lee, B.; Schatz, G.C.; Mirkin, C.A. Using DNA to design plasmonic metamaterials with tunable optical properties. Adv. Mater. 2014, 26, 653-659.

199. Xu, X.; Han, M.S.; Mirkin, C.A. A gold-nanoparticle-based real-time colorimetric screening method for endonuclease activity and inhibition. Angew. Chem. Int. Ed. 2007, 46,3468-3470. 
200. Zhao, Q.; Huang, H.; Zhang, L.; Wang, L.; Zeng, Y.; Xia, X.; Liu, F.; Chen, Y. Strategy to fabricate naked-eye readout ultrasensitive plasmonic nanosensor based on enzyme mimetic gold nanoclusters. Anal. Chem. 2016, 88, 1412-1418.

201. Cui, H.-F.; Xu, T.-B.; Sun, Y.-L.; Zhou, A.-W.; Cui, Y.-H.; Liu, W.; Luong, J.H. Hairpin DNA as a biobarcode modified on gold nanoparticles for electrochemical DNA detection. Anal. Chem. 2015, 87, 1358-1365.

202. Colombo, M.; Mazzucchelli, S.; Collico, V.; Avvakumova, S.; Pandolfi, L.; Corsi, F.; Porta, F.; Prosperi, D. Protein-Assisted One-Pot Synthesis and Biofunctionalization of Spherical Gold Nanoparticles for Selective Targeting of Cancer Cells. Angew. Chem. Int. Ed. 2012, 51, 9272-9275.

203. Sun, J.; Xianyu, Y.; Jiang, X. Point-of-care biochemical assays using gold nanoparticle-implemented microfluidics. Chem. Soc. Rev. 2014, 43, 6239-6253.

204. Chen, Y.; Xianyu, Y.; Jiang, X. Surface Modification of Gold Nanoparticles with Small Molecules for Biochemical Analysis. Acc. Chem. Res. 2017, 50, 310-319.

205. Liu, D.; Wang, Z.; Jiang, X. Gold nanoparticles for the colorimetric and fluorescent detection of ions and small organic molecules. Nanoscale 2011, 3, 1421-1433.

206. Li, N.; Binder, W.H. Click-chemistry for nanoparticle-modification. J. Mater. Chem. 2011, 21, 16717-16734.

207. Xie, R.; Hong, S.; Feng, L.; Rong, J.; Chen, X. Cell-selective metabolic glycan labeling based on ligand-targeted liposomes. J. Am. Chem. Soc. 2012, 134, 9914-9917.

208. Colombo, M.; Sommaruga, S.; Mazzucchelli, S.; Polito, L.; Verderio, P.; Galeffi, P.; Corsi, F.; Tortora, P.; Prosperi, D. Site-specific conjugation of ScFvs antibodies to nanoparticles by bioorthogonal strain-promoted alkyne-nitrone cycloaddition. Angew. Chem. 2012, 124, 511-514.

209. Lin, L.; Tian, X.; Hong, S.; Dai, P.; You, Q.; Wang, R.; Feng, L.; Xie, C.; Tian, Z.Q.; Chen, X. A bioorthogonal Raman reporter strategy for SERS detection of glycans on live cells. Angew. Chem. Int. Ed. 2013, 52, 7266-7271.

210. Ramil, C.P.; Lin, Q. Bioorthogonal chemistry: Strategies and recent developments. Chem. Commun. 2013, 49, 11007-11022.

211. Alonso, F.; Moglie, Y.; Radivoy, G. Copper nanoparticles in click chemistry. Acc. Chem. Res. 2015, 48, $2516-2528$.

212. Wang, Q.; Chan, T.R.; Hilgraf, R.; Fokin, V.V.; Sharpless, K.B.; Finn, M. Bioconjugation by copper (I)-catalyzed azide-alkyne [ $\left.{ }^{3+} 2\right]$ cycloaddition. J. Am. Chem. Soc. 2003, 125, 3192-3193.

213. Zhou, Y.; Wang, S.; Zhang, K.; Jiang, X. Visual detection of copper (II) by azide-and alkyne-functionalized gold nanoparticles using click chemistry. Angew. Chem. Int. Ed. 2008, 47, 7454-7456.

214. Zhu, K.; Zhang, Y.; He, S.; Chen, W.; Shen, J.; Wang, Z.; Jiang, X. Quantification of proteins by functionalized gold nanoparticles using click chemistry. Anal. Chem. 2012, 84, 4267-4270.

215. Qu, W.; Liu, Y.; Liu, D.; Wang, Z.; Jiang, X. Copper-Mediated Amplification Allows Readout of Immunoassays by the Naked Eye. Angew. Chem. Int. Ed. 2011, 50, 3442-3445.

216. Xianyu, Y.; Wang, Z.; Jiang, X. A plasmonic nanosensor for immunoassay via enzyme-triggered click chemistry. Acs Nano 2014, 8 , 12741-12747.

217. Xianyu, Y.; Wang, Z.; Sun, J.; Wang, X.; Jiang, X. Colorimetric logic gates through molecular recognition and plasmonic nanoparticles. Small 2014, 10, 4833-4838.

218. Zhang, L.; Feng, Q.; Wang, J.; Sun, J.; Shi, X.; Jiang, X. Microfluidic synthesis of rigid nanovesicles for hydrophilic reagents delivery. Angew. Chem. 2015, 127, 4024-4028.

219. Xianyu, Y.; Xie, Y.; Wang, N.; Wang, Z.; Jiang, X. A dispersion-dominated chromogenic strategy for colorimetric sensing of glutathione at the nanomolar level using gold nanoparticles. Small 2015, 11, 5510-5514.

220. Guo, Y.; Zhang, Y.; Shao, H.; Wang, Z.; Wang, X.; Jiang, X. Label-free colorimetric detection of cadmium ions in rice samples using gold nanoparticles. Anal. Chem. 2014, 86, 8530-8534.

221. Liu, D.; Chen, W.; Sun, K.; Deng, K.; Zhang, W.; Wang, Z.; Jiang, X. Resettable, multi-readout logic gates based on controllably reversible aggregation of gold nanoparticles. Angew. Chem. 2011, 123, 4189-4193.

222. Chen, W.; Cao, F.; Zheng, W.; Tian, Y.; Xianyu, Y.; Xu, P.; Zhang, W.; Wang, Z.; Deng, K.; Jiang, X. Detection of the nanomolar level of total $\mathrm{Cr}[(\mathrm{III})$ and $(\mathrm{VI})]$ by functionalized gold nanoparticles and a smartphone with the assistance of theoretical calculation models. Nanoscale 2015, 7, 2042-2049.

223. Zheng, W.; Li, H.; Chen, W.; Ji, J.; Jiang, X. Recyclable colorimetric detection of trivalent cations in aqueous media using zwitterionic gold nanoparticles. Anal. Chem. 2016, 88, 4140-4146.

224. Liu, D.; Qu, W.; Chen, W.; Zhang, W.; Wang, Z.; Jiang, X. Highly sensitive, colorimetric detection of mercury (II) in aqueous media by quaternary ammonium group-capped gold nanoparticles at room temperature. Anal. Chem. 2010, 82, 9606-9610.

225. Liu, D.; Wang, S.; Swierczewska, M.; Huang, X.; Bhirde, A.A.; Sun, J.; Wang, Z.; Yang, M.; Jiang, X.; Chen, X. Highly robust, recyclable displacement assay for mercuric ions in aqueous solutions and living cells. Acs Nano 2012, 6, 10999-11008.

226. Liu, D.; Chen, W.; Tian, Y.; He, S.; Zheng, W.; Sun, J.; Wang, Z.; Jiang, X. A highly sensitive gold-nanoparticle-based assay for acetylcholinesterase in cerebrospinal fluid of transgenic mice with Alzheimer's disease. Adv. Healthc. Mater. 2012, 1, 90-95.

227. Liu, D.; Chen, W.; Wei, J.; Li, X.; Wang, Z.; Jiang, X. A highly sensitive, dual-readout assay based on gold nanoparticles for organophosphorus and carbamate pesticides. Anal. Chem. 2012, 84, 4185-4191.

228. Watson, J.D.; Crick, F.H. Molecular structure of nucleic acids: A structure for deoxyribose nucleic acid. Nature 1953, 171, 737-738.

229. Alivisatos, A.P.; Johnsson, K.P.; Peng, X.; Wilson, T.E.; Loweth, C.J.; Bruchez, M.P.; Schultz, P.G. Organization of'nanocrystal molecules' using DNA. Nature 1996, 382, 609-611. 
230. Pinto, Y.Y.; Le, J.D.; Seeman, N.C.; Musier-Forsyth, K.; Taton, T.A.; Kiehl, R.A. Sequence-encoded self-assembly of multiplenanocomponent arrays by 2D DNA scaffolding. Nano Lett. 2005, 5, 2399-2402.

231. Zheng, J.; Constantinou, P.E.; Micheel, C.; Alivisatos, A.P.; Kiehl, R.A.; Seeman, N.C. Two-dimensional nanoparticle arrays show the organizational power of robust DNA motifs. Nano Lett. 2006, 6, 1502-1504.

232. Sharma, J.; Chhabra, R.; Liu, Y.; Ke, Y.; Yan, H. DNA-templated self-assembly of two-dimensional and periodical gold nanoparticle arrays. Angew. Chem. 2006, 118, 744-749.

233. Lee, J.H.; Wernette, D.P.; Yigit, M.V.; Liu, J.; Wang, Z.; Lu, Y. Site-specific control of distances between gold nanoparticles using phosphorothioate anchors on DNA and a short bifunctional molecular fastener. Angew. Chem. 2007, 119, 9164-9168.

234. Ludueña, R.F.; Roach, M.C.; Trcka, P.P.; Weintraub, S. N, N-Bis ( $\alpha$-iodoacetyl)-2, $2^{\prime}$-dithiobis (ethylamine), a reversible crosslinking reagent for protein sulfhydryl groups. Anal. Biochem. 1981, 117, 76-80.

235. Ozbay, E. Plasmonics: Merging photonics and electronics at nanoscale dimensions. Science 2006, 311, $189-193$.

236. Liu, Z.; Searson, P.C. Single nanoporous gold nanowire sensors. J. Phys. Chem. B 2006, 110, 4318-4322.

237. Chirea, M.; Freitas, A.; Vasile, B.S.; Ghitulica, C.; Pereira, C.M.; Silva, F. Gold nanowire networks: Synthesis, characterization, and catalytic activity. Langmuir 2011, 27, 3906-3913.

238. Bai, X.; Gao, Y.; Liu, H.-G.; Zheng, L. Synthesis of amphiphilic ionic liquids terminated gold nanorods and their superior catalytic activity for the reduction of nitro compounds. J. Phys. Chem. C 2009, 113, 17730-17736.

239. Richter, J. Metallization of DNA. Phys. E Low Dimens. Syst. Nanostruct. 2003, 16, 157-173.

240. Ongaro, A.; Griffin, F.; Beecher, P.; Nagle, L.; Iacopino, D.; Quinn, A.; Redmond, G.; Fitzmaurice, D. DNA-templated assembly of conducting gold nanowires between gold electrodes on a silicon oxide substrate. Chem. Mater. 2005, 17, $1959-1964$.

241. Kim, H.J.; Roh, Y.; Hong, B. Selective formation of a latticed nanostructure with the precise alignment of DNA-templated gold nanowires. Langmuir 2010, 26, 18315-18319.

242. Harnack, O.; Ford, W.E.; Yasuda, A.; Wessels, J.M. Tris (hydroxymethyl) phosphine-capped gold particles templated by DNA as nanowire precursors. Nano Lett. 2002, 2, 919-923.

243. Yonezawa, T.; Onoue, S.-Y.; Kimizuka, N. Metal coating of DNA molecules by cationic, metastable gold nanoparticles. Chem. Lett. 2002, 31, 1172-1173.

244. Patolsky, F.; Weizmann, Y.; Lioubashevski, O.; Willner, I. Au-nanoparticle nanowires based on DNA and polylysine templates. Angew. Chem. Int. Ed. 2002, 41, 2323-2327.

245. Haruta, M. Catalysis of gold nanoparticles deposited on metal oxides. Cattech 2002, 6, 102-115.

246. Fu, T.J.; Seeman, N.C. DNA double-crossover molecules. Biochemistry 1993, 32, 3211-3220.

247. Ding, B.; Sha, R.; Seeman, N.C. Pseudohexagonal 2D DNA crystals from double crossover cohesion. J. Am. Chem. Soc. 2004, 126, 10230-10231.

248. Le, J.D.; Pinto, Y.; Seeman, N.C.; Musier-Forsyth, K.; Taton, T.A.; Kiehl, R.A. DNA-templated self-assembly of metallic nanocomponent arrays on a surface. Nano Lett. 2004, 4, 2343-2347.

249. Mastroianni, A.J.; Claridge, S.A.; Alivisatos, A.P. Pyramidal and chiral groupings of gold nanocrystals assembled using DNA scaffolds. J. Am. Chem. Soc. 2009, 131, 8455-8459.

250. Chen, J.; Seeman, N.C. Synthesis from DNA of a molecule with the connectivity of a cube. Nature 1991, 350, 631-633.

251. Erben, C.M.; Goodman, R.P.; Turberfield, A.J. A self-assembled DNA bipyramid. J. Am. Chem. Soc. 2007, 129, $6992-6993$.

252. Zhang, Y.; Seeman, N.C. Construction of a DNA-truncated octahedron. J. Am. Chem. Soc. 1994, 116, $1661-1669$.

253. Riordan, T.H. A Brief Overview of DNA Origami and Its Applications; Duke University: Durham, NC, USA.

254. Andersen, E.S.; Dong, M.; Nielsen, M.M.; Jahn, K.; Subramani, R.; Mamdouh, W.; Golas, M.M.; Sander, B.; Stark, H.; Oliveira, C.L. Self-assembly of a nanoscale DNA box with a controllable lid. Nature 2009, 459, 73-76.

255. Kuzuya, A.; Komiyama, M. Design and construction of a box-shaped 3D-DNA origami. Chem. Commun. 2009, $4182-4184$.

256. Kuzyk, A.; Schreiber, R.; Fan, Z.; Pardatscher, G.; Roller, E.-M.; Högele, A.; Simmel, F.C.; Govorov, A.O.; Liedl, T. DNA-based self-assembly of chiral plasmonic nanostructures with tailored optical response. Nature 2012, 483, 311-314.

257. Yang, C.; Manocchi, A.K.; Lee, B.; Yi, H. Viral templated palladium nanocatalysts for dichromate reduction. Appl. Catal. B Environ. 2010, 93, 282-291.

258. Nam, Y.S.; Magyar, A.P.; Lee, D.; Kim, J.-W.; Yun, D.S.; Park, H.; Pollom, T.S.; Weitz, D.A.; Belcher, A.M. Biologically templated photocatalytic nanostructures for sustained light-driven water oxidation. Nat. Nanotechnol. 2010, 5, 340-344.

259. Scheibel, T.; Parthasarathy, R.; Sawicki, G.; Lin, X.-M.; Jaeger, H.; Lindquist, S.L. Conducting nanowires built by controlled self-assembly of amyloid fibers and selective metal deposition. Proc. Natl. Acad. Sci. USA 2003, 100, 4527-4532.

260. Glover, J.R.; Kowal, A.S.; Schirmer, E.C.; Patino, M.M.; Liu, J.-J.; Lindquist, S. Self-seeded fibers formed by Sup35, the protein determinant of $\left[\mathrm{PSI}^{+}\right]$, a heritable prion-like factor of S. cerevisiae. Cell 1997, 89, 811-819.

261. Steinmetz, N.F.; Evans, D.J. Utilisation of plant viruses in bionanotechnology. Org. Biomol. Chem. 2007, 5, $2891-2902$.

262. Lin, T.; Chen, Z.; Usha, R.; Stauffacher, C.V.; Dai, J.-B.; Schmidt, T.; Johnson, J.E. The refined crystal structure of cowpea mosaic virus at $2.8 \AA$ resolution. Virology 1999, 265, 20-34.

263. Steinmetz, N.F.; Lomonossoff, G.P.; Evans, D.J. Cowpea mosaic virus for material fabrication: Addressable carboxylate groups on a programmable nanoscaffold. Langmuir 2006, 22, 3488-3490.

264. Steinmetz, N.F.; Lomonossoff, G.P.; Evans, D.J. Decoration of cowpea mosaic virus with multiple, redox-active, organometallic complexes. Small 2006, 2, 530-533. 
265. Blum, A.S.; Soto, C.M.; Wilson, C.D.; Cole, J.D.; Kim, M.; Gnade, B.; Chatterji, A.; Ochoa, W.F.; Lin, T.; Johnson, J.E. Cowpea mosaic virus as a scaffold for 3-D patterning of gold nanoparticles. Nano Lett. 2004, 4, 867-870.

266. Zafeiratos, S.; Piccinin, S.; Teschner, D. Alloys in catalysis: Phase separation and surface segregation phenomena in response to the reactive environment. Catal. Sci. Technol. 2012, 2, 1787-1801.

267. Evans, D.J. The bionanoscience of plant viruses: Templates and synthons for new materials. J. Mater. Chem. 2008, 18, 3746-3754.

268. Shenton, W.; Douglas, T.; Young, M.; Stubbs, G.; Mann, S. Inorganic-organic nanotube composites from template mineralization of tobacco mosaic virus. Adv. Mater. 1999, 11, 253-256.

269. Knez, M.; Kadri, A.; Wege, C.; Gösele, U.; Jeske, H.; Nielsch, K. Atomic layer deposition on biological macromolecules: Metal oxide coating of tobacco mosaic virus and ferritin. Nano Lett. 2006, 6, 1172-1177.

270. Dujardin, E.; Peet, C.; Stubbs, G.; Culver, J.N.; Mann, S. Organization of metallic nanoparticles using tobacco mosaic virus templates. Nano Lett. 2003, 3, 413-417.

271. Lee, S.-Y.; Royston, E.; Culver, J.N.; Harris, M.T. Improved metal cluster deposition on a genetically engineered tobacco mosaic virus template. Nanotechnology 2005, 16, S435.

272. Lim, J.-S.; Kim, S.-M.; Lee, S.-Y.; Stach, E.A.; Culver, J.N.; Harris, M.T. Formation of Au/Pd alloy nanoparticles on TMV. J. Nanomater. 2010, 2010, 620505.

273. Khan, A.A.; Fox, E.K.; Górzny, M.Ł.; Nikulina, E.; Brougham, D.F.; Wege, C.; Bittner, A.M. pH control of the electrostatic binding of gold and iron oxide nanoparticles to tobacco mosaic virus. Langmuir 2013, 29, 2094-2098.

274. Guli, M.; Lambert, E.M.; Li, M.; Mann, S. Template-Directed Synthesis of Nanoplasmonic Arrays by Intracrystalline Metalization of Cross-Linked Lysozyme Crystals. Angew. Chem. 2010, 122, 530-533.

275. Wei, H.; Wang, Z.; Zhang, J.; House, S.; Gao, Y.-G.; Yang, L.; Robinson, H.; Tan, L.H.; Xing, H.; Hou, C. Time-dependent, protein-directed growth of gold nanoparticles within a single crystal of lysozyme. Nat. Nanotechnol. 2011, 6, 93.

276. Wei, H.; Lu, Y. Catalysis of gold nanoparticles within lysozyme single crystals. Chem. Asian J. 2012, 7, 680-683.

277. McMillan, R.A.; Paavola, C.D.; Howard, J.; Chan, S.L.; Zaluzec, N.J.; Trent, J.D. Ordered nanoparticle arrays formed on engineered chaperonin protein templates. Nat. Mater. 2002, 1, 247-252.

278. Trent, J.D.; Kagawa, H.K.; Yaoi, T.; Olle, E.; Zaluzec, N.J. Chaperonin filaments: The archaeal cytoskeleton? Proc. Natl. Acad. Sci. USA 1997, 94, 5383-5388.

279. Ellis, M.J.; Knapp, S.; Koeck, P.J.; Fakoor-Biniaz, Z.; Ladenstein, R.; Hebert, H. Two-Dimensional Crystallization of the Chaperonin TF55 from the Hyperthermophilic ArchaeonSulfolobus solfataricus. J. Struct. Biol. 1998, 123, 30-36.

280. Bond, G.; Louis, C.; Thompson, D. Catalysis by Gold Imperial; College Press: Joplin, MO, USA, 2006.

281. Hashmi, A.S.K.; Hutchings, G.J. Gold catalysis. Angew. Chem. Int. Ed. 2006, 45, 7896-7936.

282. Della Pina, C.; Falletta, E.; Rossi, M. Update on selective oxidation using gold. Chem. Soc. Rev. 2012, 41, 350-369.

283. Mills, G.; Gordon, M.S.; Metiu, H. Oxygen adsorption on Au clusters and a rough Au (111) surface: The role of surface flatness, electron confinement, excess electrons, and band gap. J. Chem. Phys. 2003, 118, 4198-4205.

284. Bond, G.C.; Thompson, D.T. Catalysis by gold. Catal. Rev. 1999, 41, 319-388.

285. Wales, D.J. Structure, dynamics, and thermodynamics of clusters: Tales from topographic potential surfaces. Science 1996, 271, 925-929.

286. Boyen, H.-G.; Kästle, G.; Weigl, F.; Koslowski, B.; Dietrich, C.; Ziemann, P.; Spatz, J.P.; Riethmüller, S.; Hartmann, C.; Möller, M. Oxidation-resistant gold-55 clusters. Science 2002, 297, 1533-1536.

287. Bond, G.C.; Thompson, D.T. Gold-catalysed oxidation of carbon monoxide. Gold Bull. 2000, 33, 41-50.

288. Benkovic, S.J.; Hammes-Schiffer, S. A perspective on enzyme catalysis. Science 2003, 301, 1196-1202.

289. Comotti, M.; Della Pina, C.; Matarrese, R.; Rossi, M. The catalytic activity of "naked" gold particles. Angew. Chem. Int. Ed. 2004, $43,5812-5815$.

290. Boronat, M.; Corma, A. Oxygen activation on gold nanoparticles: Separating the influence of particle size, particle shape and support interaction. Dalton Trans. 2010, 39, 8538-8546.

291. Kotov, N.A. Inorganic nanoparticles as protein mimics. Science 2010, 330, 188-189.

292. Witt, S.; Wohlfahrt, G.; Schomburg, D.; Hecht, H.-J.; Kalisz, H.M. Conserved arginine-516 of Penicillium amagasakiense glucose oxidase is essential for the efficient binding of $\beta$-D-glucose. Biochem. J. 2000, 347, 553-559.

293. Wild, S.; Roglic, G.; Green, A.; Sicree, R.; King, H. Global prevalence of diabetes: Estimates for the year 2000 and projections for 2030. Diabetes Care 2004, 27, 1047-1053.

294. Zheng, X.; Liu, Q.; Jing, C.; Li, Y.; Li, D.; Luo, W.; Wen, Y.; He, Y.; Huang, Q.; Long, Y.T. Catalytic gold nanoparticles for nanoplasmonic detection of DNA hybridization. Angew. Chem. 2011, 123, 12200-12204.

295. Newman, J.D.; Turner, A.P. Home blood glucose biosensors: A commercial perspective. Biosens. Bioelectron. 2005, 20, $2435-2453$.

296. Beltrame, P.; Comotti, M.; Della Pina, C.; Rossi, M. Aerobic oxidation of glucose: II. Catalysis by colloidal gold. Appl. Catal. A Gen. 2006, 297, 1-7.

297. Biella, S.; Prati, L.; Rossi, M. Selective oxidation of D-glucose on gold catalyst. J. Catal. 2002, 206, $242-247$.

298. Luo, W.; Zhu, C.; Su, S.; Li, D.; He, Y.; Huang, Q.; Fan, C. Self-catalyzed, self-limiting growth of glucose oxidase-mimicking gold nanoparticles. Acs Nano 2010, 4, 7451-7458. 
299. Cedervall, T.; Lynch, I.; Lindman, S.; Berggård, T.; Thulin, E.; Nilsson, H.; Dawson, K.A.; Linse, S. Understanding the nanoparticleprotein corona using methods to quantify exchange rates and affinities of proteins for nanoparticles. Proc. Natl. Acad. Sci. USA 2007, 104, 2050-2055.

300. Lacerda, S.H.D.P.; Park, J.J.; Meuse, C.; Pristinski, D.; Becker, M.L.; Karim, A.; Douglas, J.F. Interaction of gold nanoparticles with common human blood proteins. Acs Nano 2010, 4, 365-379.

301. Röcker, C.; Pötzl, M.; Zhang, F.; Parak, W.J.; Nienhaus, G.U. A quantitative fluorescence study of protein monolayer formation on colloidal nanoparticles. Nat. Nanotechnol. 2009, 4, 577-580.

302. Babitzke, P.; Gollnick, P. Posttranscription Initiation Control of Tryptophan Metabolism in Bacillus subtilis by thetrp RNA-Binding Attenuation Protein (TRAP), anti-TRAP, and RNA Structure. J. Bacteriol. 2001, 183, 5795-5802.

303. Chen, X.-p.; Antson, A.A.; Yang, M.; Li, P.; Baumann, C.; Dodson, E.J.; Dodson, G.G.; Gollnick, P. Regulatory features of the trp operon and the crystal structure of the trp RNA-binding attenuation protein from Bacillus stearothermophilus. J. Mol. Biol. 1999, 289, 1003-1016.

304. Antson, A.A.; Otridge, J.; Brzozowski, A.M.; Dodson, E.J.; Dodson, G.G.; Wilson, K.S.; Smith, T.M.; Yang, M.; Kurecki, T.; Gollnick, P. The structure of trp RNA-binding attenuation protein. Nature 1995, 374, 693-700.

305. Heddle, J.G.; Okajima, T.; Scott, D.J.; Akashi, S.; Park, S.-Y.; Tame, J.R. Dynamic allostery in the ring protein TRAP. J. Mol. Biol. 2007, 371, 154-167.

306. Heddle, J.G.; Yokoyama, T.; Yamashita, I.; Park, S.-Y.; Tame, J.R. Rounding up: Engineering 12-membered rings from the cyclic 11-mer TRAP. Structure 2006, 14, 925-933.

307. Heddle, J.G.; Fujiwara, I.; Yamadaki, H.; Yoshii, S.; Nishio, K.; Addy, C.; Yamashita, I.; Tame, J.R. Using the Ring-Shaped Protein TRAP to Capture and Confine Gold Nanodots on a Surface. Small 2007, 3, 1950-1956.

308. Malay, A.D.; Heddle, J.G.; Tomita, S.; Iwasaki, K.; Miyazaki, N.; Sumitomo, K.; Yanagi, H.; Yamashita, I.; Uraoka, Y. Gold nanoparticle-induced formation of artificial protein capsids. Nano Lett. 2012, 12, 2056-2059.

309. Wittstock, A.; Zielasek, V.; Biener, J.; Friend, C.; Bäumer, M. Nanoporous gold catalysts for selective gas-phase oxidative coupling of methanol at low temperature. Science 2010, 327, 319-322.

310. Häkkinen, H. The gold-sulfur interface at the nanoscale. Nat. Chem. 2012, 4, 443.

311. Zhang, D.; Neumann, O.; Wang, H.; Yuwono, V.M.; Barhoumi, A.; Perham, M.; Hartgerink, J.D.; Wittung-Stafshede, P.; Halas, N.J. Gold nanoparticles can induce the formation of protein-based aggregates at physiological pH. Nano Lett. $2009,9,666-671$.

312. Aubin-Tam, M.-E.; Hamad-Schifferli, K. Structure and function of nanoparticle-protein conjugates. Biomed. Mater. $2008,3,034001$.

313. Treuel, L.; Malissek, M.; Gebauer, J.S.; Zellner, R. The influence of surface composition of nanoparticles on their interactions with serum albumin. ChemPhysChem 2010, 11, 3093-3099.

314. Lynch, I.; Dawson, K.A. Protein-nanoparticle interactions. Nano Today 2008, 3, 40-47.

315. Mancin, F.; Prins, L.J.; Scrimin, P. Catalysis on gold-nanoparticle-passivating monolayers. Curr. Opin. Colloid Interface Sci. 2013, 18, 61-69.

316. Pengo, P.; Polizzi, S.; Pasquato, L.; Scrimin, P. Carboxylate-imidazole cooperativity in dipeptide-functionalized gold nanoparticles with esterase-like activity. J. Am. Chem. Soc. 2005, 127, 1616-1617.

317. Mikami, Y.; Dhakshinamoorthy, A.; Alvaro, M.; Garcia, H. Catalytic activity of unsupported gold nanoparticles. Catal. Sci. Technol. 2013, 3, 58-69.

318. Manea, F.; Houillon, F.B.; Pasquato, L.; Scrimin, P. Nanozymes: Gold-nanoparticle-based transphosphorylation catalysts. Angew. Chem. 2004, 116, 6291-6295.

319. Pingoud, A.; Fuxreiter, M.; Pingoud, V.; Wende, W. Type II restriction endonucleases: Structure and mechanism. Cell. Mol. Life Sci. 2005, 62, 685.

320. Pommier, Y.; Leo, E.; Zhang, H.; Marchand, C. DNA topoisomerases and their poisoning by anticancer and antibacterial drugs. Chem. Biol. 2010, 17, 421-433.

321. Zaupa, G.; Mora, C.; Bonomi, R.; Prins, L.J.; Scrimin, P. Catalytic self-assembled monolayers on Au nanoparticles: The source of catalysis of a transphosphorylation reaction. Chem. Eur. J. 2011, 17, 4879-4889.

322. Northrop, D.B. Follow the protons: A low-barrier hydrogen bond unifies the mechanisms of the aspartic proteases. Acc. Chem. Res. 2001, 34, 790-797.

323. Hsu, M.-H.; Josephrajan, T.; Yeh, C.-S.; Shieh, D.-B.; Su, W.-C.; Hwu, J.R. Novel arylhydrazone-conjugated gold nanoparticles with DNA-cleaving ability: The first DNA-nicking nanomaterial. Bioconjugate Chem. 2007, 18, 1709-1712.

324. Hwu, J.R.; Lin, C.C.; Chuang, S.H.; King, K.Y.; Su, T.-R.; Tsay, S.-C. Aminyl and iminyl radicals from arylhydrazones in the photo-induced DNA cleavage. Bioorganic Med. Chem. 2004, 12, 2509-2515.

325. Bonomi, R.; Selvestrel, F.; Lombardo, V.; Sissi, C.; Polizzi, S.; Mancin, F.; Tonellato, U.; Scrimin, P. Phosphate diester and DNA hydrolysis by a multivalent, nanoparticle-based catalyst. J. Am. Chem. Soc. 2008, 130, 15744-15745.

326. Fillon, Y.; Verma, A.; Ghosh, P.; Ernenwein, D.; Rotello, V.M.; Chmielewski, J. Peptide ligation catalyzed by functionalized gold nanoparticles. J. Am. Chem. Soc. 2007, 129, 6676-6677.

327. Dawson, P.E.; Muir, T.W.; Clark-Lewis, I.; Kent, S. Synthesis of proteins by native chemical ligation. Science 1994, $266,776-779$.

328. Yarus, M. Getting past the RNA world: The initial Darwinian ancestor. Cold Spring Harb. Perspect. Biol. 2011,3 , a003590.

329. Silverman, S.K. Deoxyribozymes: DNA catalysts for bioorganic chemistry. Org. Biomol. Chem. 2004, 2, $2701-2706$.

330. Fiammengo, R.; Jäschke, A. Nucleic acid enzymes. Curr. Opin. Biotechnol. 2005, 16, 614-621. 
331. Lu, Y.; Liu, J. Functional DNA nanotechnology: Emerging applications of DNAzymes and aptamers. Curr. Opin. Biotechnol. 2006, $17,580-588$.

332. Zhao, W.; Lam, J.C.; Chiuman, W.; Brook, M.A.; Li, Y. Enzymatic cleavage of nucleic acids on gold nanoparticles: A generic platform for facile colorimetric biosensors. Small 2008, 4, 810-816.

333. Santoro, S.W.; Joyce, G.F. A general purpose RNA-cleaving DNA enzyme. Proc. Natl. Acad. Sci. USA 1997, 94, 4262-4266.

334. Li, J.; Lu, Y. A highly sensitive and selective catalytic DNA biosensor for lead ions. J. Am. Chem. Soc. 2000, 122, $10466-10467$.

335. Brennan, J.L.; Hatzakis, N.S.; Tshikhudo, T.R.; Dirvianskyte, N.; Razumas, V.; Patkar, S.; Vind, J.; Svendsen, A.; Nolte, R.J.; Rowan, A.E. Bionanoconjugation via click chemistry: The creation of functional hybrids of lipases and gold nanoparticles. Bioconjugate Chem. 2006, 17, 1373-1375.

336. Prati, L.; Spontoni, P.; Gaiassi, A. From renewable to fine chemicals through selective oxidation: The case of glycerol. Top. Catal. 2009, 52, 288.

337. Villa, A.; Wang, D.; Su, D.S.; Prati, L. Gold sols as catalysts for glycerol oxidation: The role of stabilizer. ChemCatChem 2009, 1, 510-514.

338. Wang, Z.; Lévy, R.; Fernig, D.G.; Brust, M. The peptide route to multifunctional gold nanoparticles. Bioconjugate Chem. 2005, 16, 497-500.

339. Xu, X.; Wang, Y.; Huo, F.; Mirkin, C.A. Asymmetric Functionalization of Gold Nanoparticles with Oligonucleotides. J. Am. Chem. Soc. 2006, 128, 9286-9287.

340. Lazarides, A.A.; Schatz, G.C. DNA-linked metal nanosphere materials: Structural basis for the optical properties. J. Phys. Chem. B 2000, 104, 460-467.

341. Dykman, L.; Khlebtsov, N. Immunological properties of gold nanoparticles. Chem Sci 2017, 8, 1719-1735.

342. Chen, Y.-S.; Hung, Y.-C.; Lin, W.-H.; Huang, G.S. Assessment of gold nanoparticles as a size-dependent vaccine carrier for enhancing the antibody response against synthetic foot-and-mouth disease virus peptide. Nanotechnology 2010, $21,195101$.

343. Connor, E.E.; Mwamuka, J.; Gole, A.; Murphy, C.J.; Wyatt, M.D. Gold nanoparticles are taken up by human cells but do not cause acute cytotoxicity. Small 2005, 1, 325-327.

344. Pelliccia, M.; Andreozzi, P.; Paulose, J.; D’Alicarnasso, M.; Cagno, V.; Donalisio, M.; Civra, A.; Broeckel, R.M.; Haese, N.; Silva, P.J Additives for vaccine storage to improve thermal stability of adenoviruses from hours to months. Nat. Commun. 2016, 7, 1-7.

345. Zhang, J.; Mou, L.; Jiang, X. Surface chemistry of gold nanoparticles for healthrelated applications. R. Soc. Chem. 2020, 11, 923-936.

346. Gole, A.; Murphy, C.J. Azide-derivatized gold nanorods: Functional materials for "click" chemistry. Langmuir 2008, $24,266-272$.

347. Wilson, C.G.; Sisco, P.N.; Gadala-Maria, F.A.; Murphy, C.J.; Goldsmith, E.C. Polyelectrolyte-coated gold nanorods and their interactions with type I collagen. Biomaterials 2009, 30, 5639-5648.

348. Niikura, K.; Matsunaga, T.; Suzuki, T.; Kobayashi, S.; Yamaguchi, H.; Orba, Y.; Kawaguchi, A.; Hasegawa, H.; Kajino, K.; Ninomiya, T. Gold nanoparticles as a vaccine platform: Influence of size and shape on immunological responses in vitro and in vivo. Acs Nano 2013, 7, 3926-3938.

349. Yang, J.A.; Johnson, B.J.; Wu, S.; Woods, W.S.; George, J.M.; Murphy, C.J. Study of wild-type $\alpha$-synuclein binding and orientation on gold nanoparticles. Langmuir 2013, 29, 4603-4615.

350. Torelli, M.D.; Putans, R.A.; Tan, Y.; Lohse, S.E.; Murphy, C.J.; Hamers, R.J. Quantitative determination of ligand densities on nanomaterials by X-ray photoelectron spectroscopy. Acs Appl. Mater. Interfaces 2015, 7, 1720-1725.

351. Huang, R.; Carney, R.P.; Ikuma, K.; Stellacci, F.; Lau, B.L. Effects of surface compositional and structural heterogeneity on nanoparticle-protein interactions: Different protein configurations. Acs Nano 2014, 8, 5402-5412.

352. Hong, R.; Fischer, N.O.; Verma, A.; Goodman, C.M.; Emrick, T.; Rotello, V.M. Control of protein structure and function through surface recognition by tailored nanoparticle scaffolds. J. Am. Chem. Soc. 2004, 126, 739-743.

353. Kumar, R.; Ray, P.C.; Datta, D.; Bansal, G.P.; Angov, E.; Kumar, N. Nanovaccines for malaria using Plasmodium falciparum antigen Pfs25 attached gold nanoparticles. Vaccine 2015, 33, 5064-5071.

354. Chen, H.; Zhang, J.; Gao, Y.; Liu, S.; Koh, K.; Zhu, X.; Yin, Y. Sensitive cell apoptosis assay based on caspase-3 activity detection with graphene oxide-assisted electrochemical signal amplification. Biosens. Bioelectron. 2015, 68, 777-782.

355. Fischer, M. Surface plasmon resonance: A general introduction. Methods Mol. Biol. 2010, 627, 1-14.

356. Stone, J.W.; Thornburg, N.J.; Blum, D.L.; Kuhn, S.J.; Wright, D.W.; Crowe, J.E., Jr. Gold nanorod vaccine for respiratory syncytial virus. Nanotechnology 2013, 24, 295102.

357. Almeida, J.P.M.; Lin, A.Y.; Figueroa, E.R.; Foster, A.E.; Drezek, R.A. In vivo gold nanoparticle delivery of peptide vaccine induces anti-tumor immune response in prophylactic and therapeutic tumor models. Small 2015, 11, 1453-1459.

358. Chen, H.; Zhang, J.; Liu, X.; Gao, Y.; Ye, Z.; Li, G. Colorimetric copper (II) ion sensor based on the conformational change of peptide immobilized onto the surface of gold nanoparticles. Anal. Methods 2014, 6, 2580-2585.

359. Gao, Y.; Zou, F.; Wu, B.; Wang, X.; Zhang, J.; Koh, K.; Chen, H. CB [7]-mediated signal amplification approach for sensitive surface plasmon resonance spectroscopy. Biosens. Bioelectron. 2016, 81, 207-213.

360. Tao, W.; Ziemer, K.S.; Gill, H.S. Gold nanoparticle-M2e conjugate coformulated with CpG induces protective immunity against influenza A virus. Nanomedicine 2014, 9, 237-251. 
361. Brinas, R.P.; Sundgren, A.; Sahoo, P.; Morey, S.; Rittenhouse-Olson, K.; Wilding, G.E.; Deng, W.; Barchi, J.J., Jr. Design and synthesis of multifunctional gold nanoparticles bearing tumor-associated glycopeptide antigens as potential cancer vaccines. Bioconjugate Chem. 2012, 23, 1513-1523.

362. Yu, X.; Feizpour, A.; Ramirez, N.-G.P.; Wu, L.; Akiyama, H.; Xu, F.; Gummuluru, S.; Reinhard, B.M. Glycosphingolipidfunctionalized nanoparticles recapitulate CD169-dependent HIV-1 uptake and trafficking in dendritic cells. Nat. Commun. 2014, $5,1-12$.

363. Doores, K.J.; Bonomelli, C.; Harvey, D.J.; Vasiljevic, S.; Dwek, R.A.; Burton, D.R.; Crispin, M.; Scanlan, C.N. Envelope glycans of immunodeficiency virions are almost entirely oligomannose antigens. Proc. Natl. Acad. Sci. USA 2010, 107, $13800-13805$.

364. Marradi, M.; Di Gianvincenzo, P.; Enríquez-Navas, P.M.; Martínez-Ávila, O.M.; Chiodo, F.; Yuste, E.; Angulo, J.; Penadés, S. Gold nanoparticles coated with oligomannosides of HIV-1 glycoprotein gp120 mimic the carbohydrate epitope of antibody $2 \mathrm{G} 12$. J. Mol. Biol. 2011, 410, 798-810.

365. Parry, A.L.; Clemson, N.A.; Ellis, J.; Bernhard, S.S.; Davis, B.G.; Cameron, N.R. 'Multicopy multivalent'glycopolymer-stabilized gold nanoparticles as potential synthetic cancer vaccines. J. Am. Chem. Soc. 2013, 135, 9362-9365.

366. Tian, Y.; Wang, H.; Liu, Y.; Mao, L.; Chen, W.; Zhu, Z.; Liu, W.; Zheng, W.; Zhao, Y.; Kong, D. A peptide-based nanofibrous hydrogel as a promising DNA nanovector for optimizing the efficacy of HIV vaccine. Nano Lett. 2014, 14, $1439-1445$.

367. Xu, L.; Liu, Y.; Chen, Z.; Li, W.; Liu, Y.; Wang, L.; Liu, Y.; Wu, X.; Ji, Y.; Zhao, Y. Surface-engineered gold nanorods: Promising DNA vaccine adjuvant for HIV-1 treatment. Nano Lett. 2012, 12, 2003-2012.

368. Zhou, X.; Zhang, X.; Yu, X.; Zha, X.; Fu, Q.; Liu, B.; Wang, X.; Chen, Y.; Chen, Y.; Shan, Y. The effect of conjugation to gold nanoparticles on the ability of low molecular weight chitosan to transfer DNA vaccine. Biomaterials 2008, 29, 111-117.

369. Zhou, W.; Gao, X.; Liu, D.; Chen, X. Gold nanoparticles for in vitro diagnostics. Chem. Rev. 2015, 115, 10575-10636.

370. Guo, Y.; Wang, Z.; Qu, W.; Shao, H.; Jiang, X. Colorimetric detection of mercury, lead and copper ions simultaneously using protein-functionalized gold nanoparticles. Biosens. Bioelectron. 2011, 26, 4064-4069.

371. Miranda, O.R.; Li, X.; Garcia-Gonzalez, L.; Zhu, Z.-J.; Yan, B.; Bunz, U.H.; Rotello, V.M. Colorimetric bacteria sensing using a supramolecular enzyme-nanoparticle biosensor. J. Am. Chem. Soc. 2011, 133, 9650-9653.

372. Lien, C.-W.; Tseng, Y.-T.; Huang, C.-C.; Chang, H.-T. Logic control of enzyme-like gold nanoparticles for selective detection of lead and mercury ions. Anal. Chem. 2014, 86, 2065-2072.

373. Kim, Y.; Johnson, R.C.; Hupp, J.T. Gold nanoparticle-based sensing of "spectroscopically silent" heavy metal ions. Nano Lett. 2001, $1,165-167$.

374. Xu, X.; Daniel, W.L.; Wei, W.; Mirkin, C.A. Colorimetric $\mathrm{Cu}^{2+}$ detection using DNA-modified gold-nanoparticle aggregates as probes and click chemistry. Small 2010, 6, 623-626.

375. Wang, C.-I.; Huang, C.-C.; Lin, Y.-W.; Chen, W.-T.; Chang, H.-T. Catalytic gold nanoparticles for fluorescent detection of mercury (II) and lead (II) ions. Anal. Chim. Acta 2012, 745, 124-130.

376. Lin, Y.-W.; Huang, C.-C.; Chang, H.-T. Gold nanoparticle probes for the detection of mercury, lead and copper ions. Analyst 2011, $136,863-871$.

377. Lien, C.-W.; Chen, Y.-C.; Chang, H.-T.; Huang, C.-C. Logical regulation of the enzyme-like activity of gold nanoparticles by using heavy metal ions. Nanoscale 2013, 5, 8227-8234.

378. Tsai, C.-S.; Yu, T.-B.; Chen, C.-T. Gold nanoparticle-based competitive colorimetric assay for detection of protein-protein interactions. Chem. Commun. 2005, 4273-4275.

379. Pavlov, V.; Xiao, Y.; Shlyahovsky, B.; Willner, I. Aptamer-functionalized Au nanoparticles for the amplified optical detection of thrombin. J. Am. Chem. Soc. 2004, 126, 11768-11769.

380. Wang, X.; Ramstrom, O.; Yan, M. Quantitative analysis of multivalent ligand presentation on gold glyconanoparticles and the impact on lectin binding. Anal. Chem. 2010, 82, 9082-9089.

381. Xianyu, Y.; Chen, Y.; Jiang, X. Horseradish peroxidase-mediated, iodide-catalyzed cascade reaction for plasmonic immunoassays. Anal. Chem. 2015, 87, 10688-10692.

382. Zhang, J.; Zheng, W.; Jiang, X. Ag ${ }^{+}$-Gated Surface Chemistry of Gold Nanoparticles and Colorimetric Detection of Acetylcholinesterase. Small 2018, 14, 1801680.

383. Wang, C.-I.; Chen, W.-T.; Chang, H.-T. Enzyme mimics of Au/Ag nanoparticles for fluorescent detection of acetylcholine. Anal. Chem. 2012, 84, 9706-9712.

384. Yang, M.; Zhang, W.; Zheng, W.; Cao, F.; Jiang, X. Inkjet-printed barcodes for a rapid and multiplexed paper-based assay compatible with mobile devices. Lab Chip 2017, 17, 3874-3882.

385. Chen, Y.; Sun, J.; Xianyu, Y.; Yin, B.; Niu, Y.; Wang, S.; Cao, F.; Zhang, X.; Wang, Y.; Jiang, X. A dual-readout chemiluminescent-gold lateral flow test for multiplex and ultrasensitive detection of disease biomarkers in real samples. Nanoscale 2016, 8, 15205-15212.

386. Deng, J.; Yang, M.; Wu, J.; Zhang, W.; Jiang, X. A self-contained chemiluminescent lateral flow assay for point-of-care testing. Anal. Chem. 2018, 90, 9132-9137.

387. Yang, M.; Zhang, W.; Yang, J.; Hu, B.; Cao, F.; Zheng, W.; Chen, Y.; Jiang, X. Skiving stacked sheets of paper into test paper for rapid and multiplexed assay. Sci. Adv. 2017, 3, eaao4862.

388. Chen, J.; Jackson, A.A.; Rotello, V.M.; Nugen, S.R. Colorimetric Detection of Escherichia coli Based on the Enzyme-Induced Metallization of Gold Nanorods. Small 2016, 12, 2469-2475. 
389. Wei, J.; Zheng, L.; Lv, X.; Bi, Y.; Chen, W.; Zhang, W.; Shi, Y.; Zhao, L.; Sun, X.; Wang, F. Analysis of influenza virus receptor specificity using glycan-functionalized gold nanoparticles. Acs Nano 2014, 8, 4600-4607.

390. Yang, X.; Dang, Y.; Lou, J.; Shao, H.; Jiang, X. D-alanyl-D-alanine-modified gold nanoparticles form a broad-spectrum sensor for bacteria. Theranostics 2018, 8, 1449.

391. Miller, K.P.; Wang, L.; Benicewicz, B.C.; Decho, A.W. Inorganic nanoparticles engineered to attack bacteria. Chem. Soc. Rev. 2015, 44, 7787-7807.

392. Gupta, A.; Mumtaz, S.; Li, C.-H.; Hussain, I.; Rotello, V.M. Combatting antibiotic-resistant bacteria using nanomaterials. Chem. Soc. Rev. 2019, 48, 415-427.

393. Cui, Y.; Zhao, Y.; Tian, Y.; Zhang, W.; Lü, X.; Jiang, X. The molecular mechanism of action of bactericidal gold nanoparticles on Escherichia coli. Biomaterials 2012, 33, 2327-2333.

394. Li, X.; Robinson, S.M.; Gupta, A.; Saha, K.; Jiang, Z.; Moyano, D.F.; Sahar, A.; Riley, M.A.; Rotello, V.M. Functional gold nanoparticles as potent antimicrobial agents against multi-drug-resistant bacteria. Acs Nano 2014, 8, 10682-10686.

395. Huo, S.; Jiang, Y.; Gupta, A.; Jiang, Z.; Landis, R.F.; Hou, S.; Liang, X.-J.; Rotello, V.M. Fully zwitterionic nanoparticle antimicrobial agents through tuning of core size and ligand structure. Acs Nano 2016, 10, 8732-8737.

396. Gupta, A.; Saleh, N.M.; Das, R.; Landis, R.F.; Bigdeli, A.; Motamedchaboki, K.; Campos, A.R.; Pomeroy, K.; Mahmoudi, M.; Rotello, V.M. Synergistic antimicrobial therapy using nanoparticles and antibiotics for the treatment of multidrug-resistant bacterial infection. Nano Futures 2017, 1, 015004.

397. Zhao, Y.; Chen, Z.; Chen, Y.; Xu, J.; Li, J.; Jiang, X. Synergy of non-antibiotic drugs and pyrimidinethiol on gold nanoparticles against superbugs. J. Am. Chem. Soc. 2013, 135, 12940-12943.

398. Wei, S.-C.; Chang, L.; Huang, C.-C.; Chang, H.-T. Dual-functional gold nanoparticles with antimicrobial and proangiogenic activities improve the healing of multidrug-resistant bacteria-infected wounds in diabetic mice. Biomater. Sci. 2019, 7, 4482-4490.

399. Feng, Y.; Chen, W.; Jia, Y.; Tian, Y.; Zhao, Y.; Long, F.; Rui, Y.; Jiang, X. N-Heterocyclic molecule-capped gold nanoparticles as effective antibiotics against multi-drug resistant bacteria. Nanoscale 2016, 8, 13223-13227.

400. Yang, X.; Yang, J.; Wang, L.; Ran, B.; Jia, Y.; Zhang, L.; Yang, G.; Shao, H.; Jiang, X. Pharmaceutical intermediate-modified gold nanoparticles: Against multidrug-resistant bacteria and wound-healing application via an electrospun scaffold. Acs Nano 2017, $11,5737-5745$.

401. Ju, P.; Hu, J.; Li, F.; Cao, Y.; Li, L.; Shi, D.; Hao, Y.; Zhang, M.; He, J.; Ni, P. A biodegradable polyphosphoester-functionalized poly (disulfide) nanocarrier for reduction-triggered intracellular drug delivery. J. Mater. Chem. B 2018, 6, 7263-7273.

402. Yang, X.; Wei, Q.; Shao, H.; Jiang, X. Multivalent Aminosaccharide-Based Gold Nanoparticles as Narrow-Spectrum Antibiotics in Vivo. Acs Appl. Mater. Interfaces 2019, 11, 7725-7730.

403. Zhao, X.; Jia, Y.; Li, J.; Dong, R.; Zhang, J.; Ma, C.; Wang, H.; Rui, Y.; Jiang, X. Indole derivative-capped gold nanoparticles as an effective bactericide in vivo. Acs Appl. Mater. Interfaces 2018, 10, 29398-29406.

404. Li, Y.; Tian, Y.; Zheng, W.; Feng, Y.; Huang, R.; Shao, J.; Tang, R.; Wang, P.; Jia, Y.; Zhang, J. Composites of bacterial cellulose and small molecule-decorated gold nanoparticles for treating Gram-Negative bacteria-infected wounds. Small 2017, 13, 1700130.

405. Zheng, W.; Jia, Y.; Chen, W.; Wang, G.; Guo, X.; Jiang, X. Universal coating from electrostatic self-assembly to prevent multidrugresistant bacterial colonization on medical devices and solid surfaces. Acs Appl. Mater. Interfaces 2017, 9, $21181-21189$.

406. Xie, Y.; Liu, Y.; Yang, J.; Liu, Y.; Hu, F.; Zhu, K.; Jiang, X. Gold Nanoclusters for Targeting Methicillin-Resistant Staphylococcus aureus In Vivo. Angew. Chem. Int. Ed. 2018, 57, 3958-3962.

407. Chen, W.Y.; Chang, H.Y.; Lu, J.K.; Huang, Y.C.; Harroun, S.G.; Tseng, Y.T.; Li, Y.J.; Huang, C.C.; Chang, H.T. Self-assembly of antimicrobial peptides on gold nanodots: Against multidrug-resistant bacteria and wound-healing application. Adv. Funct. Mater. 2015, 25, 7189-7199.

408. Hu, B.; Kong, F.; Gao, X.; Jiang, L.; Li, X.; Gao, W.; Xu, K.; Tang, B. Avoiding Thiol Compound Interference: A Nanoplatform Based on High-Fidelity Au-Se Bonds for Biological Applications. Angew. Chem. 2018, 130, 5404-5407.

409. Smith, C.A.; Narouz, M.R.; Lummis, P.A.; Singh, I.; Nazemi, A.; Li, C.-H.; Crudden, C.M. N-Heterocyclic carbenes in materials chemistry. Chem. Rev. 2019, 119, 4986-5056.

410. Narouz, M.R.; Osten, K.M.; Unsworth, P.J.; Man, R.W.; Salorinne, K.; Takano, S.; Tomihara, R.; Kaappa, S.; Malola, S.; Dinh, C.-T. N-heterocyclic carbene-functionalized magic-number gold nanoclusters. Nat. Chem. 2019, 11, 419-425.

411. Narouz, M.R.; Takano, S.; Lummis, P.A.; Levchenko, T.I.; Nazemi, A.; Kaappa, S.; Malola, S.; Yousefalizadeh, G.; Calhoun, L.A.; Stamplecoskie, K.G. Robust, highly luminescent Au13 superatoms protected by N-heterocyclic carbenes. J. Am. Chem. Soc. 2019, 141, 14997-15002.

412. Salorinne, K.; Man, R.W.; Li, C.H.; Taki, M.; Nambo, M.; Crudden, C.M. Water-Soluble N-Heterocyclic Carbene-Protected Gold Nanoparticles: Size-Controlled Synthesis, Stability, and Optical Properties. Angew. Chem. Int. Ed. 2017, 56, 6198-6202.

413. Dreaden, E.C.; Alkilany, A.M.; Huang, X.; Murphy, C.J.; El-Sayed, M.A. The golden age: Gold nanoparticles for biomedicine. Chem. Soc. Rev. 2012, 41, 2740-2779.

414. Giljohann, D.A.; Seferos, D.S.; Daniel, W.L.; Massich, M.D.; Patel, P.C.; Mirkin, C.A. Gold nanoparticles for biology and medicine. Angew. Chem. Int. Ed. 2010, 49, 3280-3294.

415. Hainfeld, J.F.; Slatkin, D.N.; Smilowitz, H.M. The use of gold nanoparticles to enhance radiotherapy in mice. Phys. Med. Biol. 2004, 49, N309. 
416. Her, S.; Jaffray, D.A.; Allen, C. Gold nanoparticles for applications in cancer radiotherapy: Mechanisms and recent advancements. Adv. Drug Deliv. Rev. 2017, 109, 84-101.

417. Dreaden, E.C.; Austin, L.A.; Mackey, M.A.; El-Sayed, M.A. Size matters: Gold nanoparticles in targeted cancer drug delivery. Ther. Deliv. 2012, 3, 457-478.

418. Mosquera, J.S.; Henriksen-Lacey, M.; García, I.; Martínez-Calvo, M.; Rodríguez, J.S.; Mascareñas, J.L.; Liz-Marzán, L.M. Cellular uptake of gold nanoparticles triggered by host-guest interactions. J. Am. Chem. Soc. 2018, 140, 4469-4472.

419. Mizuhara, T.; Saha, K.; Moyano, D.F.; Kim, C.S.; Yan, B.; Kim, Y.K.; Rotello, V.M. Acylsulfonamide-functionalized Zwitterionic gold nanoparticles for enhanced cellular uptake at tumor $\mathrm{pH}$. Angew. Chem. 2015, 127, 6667-6670.

420. Zhang, X.-D.; Wu, D.; Shen, X.; Chen, J.; Sun, Y.-M.; Liu, P.-X.; Liang, X.-J. Size-dependent radiosensitization of PEG-coated gold nanoparticles for cancer radiation therapy. Biomaterials 2012, 33, 6408-6419.

421. Zhang, X.D.; Chen, J.; Luo, Z.; Wu, D.; Shen, X.; Song, S.S.; Sun, Y.M.; Liu, P.X.; Zhao, J.; Huo, S. Enhanced Tumor Accumulation of Sub-2 nm Gold Nanoclusters for Cancer Radiation Therapy. Adv. Healthc. Mater. 2014, 3, 133-141.

422. Hirsch, L.R.; Stafford, R.J.; Bankson, J.A.; Sershen, S.R.; Rivera, B.; Price, R.; Hazle, J.D.; Halas, N.J.; West, J.L. Nanoshell-mediated near-infrared thermal therapy of tumors under magnetic resonance guidance. Proc. Natl. Acad. Sci. USA 2003, 100, 13549-13554.

423. Loo, C.; Lowery, A.; Halas, N.; West, J.; Drezek, R. Immunotargeted nanoshells for integrated cancer imaging and therapy. Nano Lett. 2005, 5, 709-711.

424. Alkilany, A.M.; Thompson, L.B.; Boulos, S.P.; Sisco, P.N.; Murphy, C.J. Gold nanorods: Their potential for photothermal therapeutics and drug delivery, tempered by the complexity of their biological interactions. Adv. Drug Deliv. Rev. 2012, 64, 190-199.

425. Huang, X.; El-Sayed, I.H.; Qian, W.; El-Sayed, M.A. Cancer cell imaging and photothermal therapy in the near-infrared region by using gold nanorods. J. Am. Chem. Soc. 2006, 128, 2115-2120.

426. Aioub, M.; Panikkanvalappil, S.R.; El-Sayed, M.A. Platinum-coated gold nanorods: Efficient reactive oxygen scavengers that prevent oxidative damage toward healthy, untreated cells during plasmonic photothermal therapy. Acs Nano 2017, 11, 579-586.

427. Volsi, A.L.; de Aberasturi, D.J.; Henriksen-Lacey, M.; Giammona, G.; Licciardi, M.; Liz-Marzán, L.M. Inulin coated plasmonic gold nanoparticles as a tumor-selective tool for cancer therapy. J. Mater. Chem. B 2016, 4, 1150-1155.

428. Shen, S.; Tang, H.; Zhang, X.; Ren, J.; Pang, Z.; Wang, D.; Gao, H.; Qian, Y.; Jiang, X.; Yang, W. Targeting mesoporous silicaencapsulated gold nanorods for chemo-photothermal therapy with near-infrared radiation. Biomaterials 2013, 34, 3150-3158.

429. Zhang, Z.; Wang, L.; Wang, J.; Jiang, X.; Li, X.; Hu, Z.; Ji, Y.; Wu, X.; Chen, C. Mesoporous silica-coated gold nanorods as a light-mediated multifunctional theranostic platform for cancer treatment. Adv. Mater. 2012, 24, 1418-1423.

430. Mallat, T.; Baiker, A. Oxidation of alcohols with molecular oxygen on solid catalysts. Chem. Rev. 2004, 104, 3037-3058.

431. Corma, A.; Garcia, H. Supported gold nanoparticles as catalysts for organic reactions. Chem. Soc. Rev. 2008, 37, $2096-2126$.

432. Haruta, M.; Yamada, N.; Kobayashi, T.; Iijima, S. Gold catalysts prepared by coprecipitation for low-temperature oxidation of hydrogen and of carbon monoxide. J. Catal. 1989, 115, 301-309.

433. Enache, D.I.; Edwards, J.K.; Landon, P.; Solsona-Espriu, B.; Carley, A.F.; Herzing, A.A.; Watanabe, M.; Kiely, C.J.; Knight, D.W.; Hutchings, G.J. Solvent-free oxidation of primary alcohols to aldehydes using Au-Pd/TiO2 catalysts. Science 2006, 311, 362-365.

434. Hayashi, T.; Inagaki, T.; Itayama, N.; Baba, H. Selective oxidation of alcohol over supported gold catalysts: Methyl glycolate formation from ethylene glycol and methanol. Catal. Today 2006, 117, 210-213.

435. Corma, A.; Domine, M.E. Gold supported on a mesoporous $\mathrm{CeO}_{2}$ matrix as an efficient catalyst in the selective aerobic oxidation of aldehydes in the liquid phase. Chem. Commun. 2005, 4042-4044. [CrossRef]

436. Klitgaard, S.K.; Egeblad, K.; Mentzel, U.V.; Popov, A.G.; Jensen, T.; Taarning, E.; Nielsen, I.S.; Christensen, C.H. Oxidations of amines with molecular oxygen using bifunctional gold-titania catalysts. Green Chem. 2008, 10, 419-423.

437. Haruta, M. When gold is not noble: Catalysis by nanoparticles. Chem. Rec. 2003, 3, 75-87.

438. Boronat, M.; Corma, A. Origin of the different activity and selectivity toward hydrogenation of single metal $\mathrm{Au}$ and $\mathrm{Pt}$ on $\mathrm{TiO}_{2}$ and bimetallic $\mathrm{Au}^{-} \mathrm{Pt} / \mathrm{TiO}_{2}$ catalysts. Langmuir 2010, 26, 16607-16614.

439. Zhang, Z.; Shao, C.; Zou, P.; Zhang, P.; Zhang, M.; Mu, J.; Guo, Z.; Li, X.; Wang, C.; Liu, Y. In situ assembly of well-dispersed gold nanoparticles on electrospun silica nanotubes for catalytic reduction of 4-nitrophenol. Chem. Commun. 2011, 47, 3906-3908.

440. Milone, C.; Ingoglia, R.; Tropeano, M.; Neri, G.; Galvagno, S. First example of selective hydrogenation of unconstrained $\alpha$, $\beta$-unsaturated ketone to $\alpha, \beta$-unsaturated alcohol by molecular hydrogen. Chem. Commun. 2003, 868-869.

441. Ide, M.S.; Hao, B.; Neurock, M.; Davis, R.J. Mechanistic insights on the hydrogenation of $\alpha$, $\beta$-unsaturated ketones and aldehydes to unsaturated alcohols over metal catalysts. Acs Catal. 2012, 2, 671-683.

442. Rashid, M.H.; Mandal, T.K. Templateless synthesis of polygonal gold nanoparticles: An unsupported and reusable catalyst with superior activity. Adv. Funct. Mater. 2008, 18, 2261-2271.

443. Panigrahi, S.; Basu, S.; Praharaj, S.; Pande, S.; Jana, S.; Pal, A.; Ghosh, S.K.; Pal, T. Synthesis and size-selective catalysis by supported gold nanoparticles: Study on heterogeneous and homogeneous catalytic process. J. Phys. Chem. C 2007, 111, 4596-4605.

444. Kuroda, K.; Ishida, T.; Haruta, M. Reduction of 4-nitrophenol to 4-aminophenol over Au nanoparticles deposited on PMMA. J. Mol. Catal. A Chem. 2009, 298, 7-11.

445. Abay, A.K.; Chen, X.; Kuo, D.-H. Highly efficient noble metal free copper nickel oxysulfide nanoparticles for catalytic reduction of 4-nitrophenol, methyl blue, and rhodamine-B organic pollutants. New J. Chem. 2017, 41, 5628-5638. 
446. Wu, G.; Liu, X.; Zhou, P.; Wang, L.; Hegazy, M.; Huang, X.; Huang, Y. A facile approach for the reduction of 4-nitrophenol and degradation of congo red using gold nanoparticles or laccase decorated hybrid inorganic nanoparticles/polymerbiomacromolecules vesicles. Mater. Sci. Eng. C 2019, 94, 524-553.

447. Das, S.K.; Dickinson, C.; Lafir, F.; Brougham, D.F.; Marsili, E. Synthesis, characterization and catalytic activity of gold nanoparticles biosynthesized with Rhizopus oryzae protein extract. R. Soc. Chem. 2012, 14, 1322-1334.

448. Punnoose, M.S.; Bijimol, D.; Abraham, T.; Plathanam, N.J.; Mathew, B. Synthesis, Characterization and Catalytic Activity of Gold Nanoparticles synthesized using a Green Route. AIP Conf. Proc. 2019, 2269, 030003.

449. Kumar, I.; Mondal, M.; Meyappan, V.; Sakthivel, N. Green one-pot synthesis of gold nanoparticles using Sansevieria roxburghiana leaf extract for the catalytic degradation of toxic organic pollutants. Mater. Res. Bull. 2019, 117, 18-27.

450. Kobayashi, H.; Teranishi, M.; Negishi, R.; Naya, S.-I.; Tada, H. Reaction mechanism of the multiple-electron oxygen reduction reaction on the surfaces of gold and platinum nanoparticles loaded on titanium (IV) oxide. J. Phys. Chem. Lett. 2016, 7, 5002-5007.

451. Tanaka, A.; Teramura, K.; Hosokawa, S.; Kominami, H.; Tanaka, T. Visible light-induced water splitting in an aqueous suspension of a plasmonic $\mathrm{Au} / \mathrm{TiO} 2$ photocatalyst with metal co-catalysts. Chem. Sci. 2017, 8, 2574-2580.

452. Buehler, N.; Meier, K.; Reber, J.F. Photochemical hydrogen production with cadmium sulfide suspensions. J. Phys. Chem. 1984, 88, 3261-3268.

453. Tada, H.; Mitsui, T.; Kiyonaga, T.; Akita, T.; Tanaka, K. All-solid-state Z-scheme in CdS-Au-TiO 2 three-component nanojunction system. Nat. Mater. 2006, 5, 782-786.

454. Naya, S.-i.; Kume, T.; Akashi, R.; Fujishima, M.; Tada, H. Red-light-driven water splitting by Au (core)-CdS (shell) half-cut nanoegg with heteroepitaxial junction. J. Am. Chem. Soc. 2018, 140, 1251-1254.

455. Tada, H. Overall water splitting and hydrogen peroxide synthesis by gold nanoparticle-based plasmonic photocatalysts. $R$. Soc. Chem. 2019, 1, 4238-4245.

456. Reineck, P.; Lee, G.P.; Brick, D.; Karg, M.; Mulvaney, P.; Bach, U. A solid-state plasmonic solar cell via metal nanoparticle self-assembly. Adv. Mater 2012, 24, 4750-4755.

457. Meissner, D.; Memming, R.; Bahnemann, K.D. Fundamental problems of water splitting at cadmium sulfide. Phys. Lett 1986, 127, 419.

458. Lang, X.; Chen, X.; Zhao, J. Heterogeneous visible light photocatalysis for selective organic transformations. Chem. Soc. Rev. 2014, $43,473-486$.

459. Naya, S.I.; Niwa, T.; Kume, T.; Tada, H. Visible-Light-Induced Electron Transport from Small to Large Nanoparticles in Bimodal Gold Nanoparticle-Loaded Titanium (IV) Oxide. Angew. Chem. 2014, 126, 7433-7437.

460. Li, X.; Chen, C.; Zhao, J. Mechanism of photodecomposition of $\mathrm{H}_{2} \mathrm{O}_{2}$ on $\mathrm{TiO}_{2}$ surfaces under visible light irradiation. Langmuir 2001, 17, 4118-4122.

461. Minero, C.; Mariella, G.; Maurino, V.; Pelizzetti, E. Photocatalytic transformation of organic compounds in the presence of inorganic anions. 1. Hydroxyl-mediated and direct electron-transfer reactions of phenol on a titanium dioxide-fluoride system. Langmuir 2000, 16, 2632-2641.

462. Park, H.; Choi, W. Effects of $\mathrm{TiO}_{2}$ surface fluorination on photocatalytic reactions and photoelectrochemical behaviors. J. Phys. Chem. B 2004, 108, 4086-4093.

463. Maurino, V.; Minero, C.; Mariella, G.; Pelizzetti, E. Sustained production of $\mathrm{H}_{2} \mathrm{O}_{2}$ on irradiated $\mathrm{TiO}_{2}-$ fluoride systems. Chem. Commun. 2005, 2627-2629.

464. Hirakawa, H.; Shiota, S.; Shiraishi, Y.; Sakamoto, H.; Ichikawa, S.; Hirai, T. Au nanoparticles supported on BiVO4: Effective inorganic photocatalysts for $\mathrm{H}_{2} \mathrm{O}_{2}$ production from water and $\mathrm{O}_{2}$ under visible light. Acs Catal. 2016, 6, 4976-4982.

465. Shiraishi, Y.; Kanazawa, S.; Sugano, Y.; Tsukamoto, D.; Sakamoto, H.; Ichikawa, S.; Hirai, T. Highly selective production of hydrogen peroxide on graphitic carbon nitride (g-C3N4) photocatalyst activated by visible light. Acs Catal. $2014,4,774-780$.

466. Shiraishi, Y.; Kanazawa, S.; Kofuji, Y.; Sakamoto, H.; Ichikawa, S.; Tanaka, S.; Hirai, T. Sunlight-driven hydrogen peroxide production from water and molecular oxygen by metal-free photocatalysts. Angew. Chem. Int. Ed. 2014, 53, 13454-13459.

467. Teranishi, M.; Naya, S.-I.; Tada, H. In situ liquid phase synthesis of hydrogen peroxide from molecular oxygen using gold nanoparticle-loaded titanium (IV) dioxide photocatalyst. J. Am. Chem. Soc. 2010, 132, 7850-7851.

468. Schasfoort, R.; Tudos, A. Handbook of Surface Plasmon Resonance; RSC Publishing: Cambridge, UK, 2008.

469. Link, S.; El-Sayed, M.A. Shape and size dependence of radiative, non-radiative and photothermal properties of gold nanocrystals. Int. Rev. Phys. Chem. 2000, 19, 409-453.

470. Underwood, S.; Mulvaney, P. Effect of the solution refractive index on the color of gold colloids. Langmuir 1994, 10, 3427-3430.

471. Anker, J.N.; Hall, W.P.; Lyandres, O.; Shah, N.C.; Zhao, J.; Van Duyne, R.P. Biosensing with plasmonic nanosensors. Nanosci. Technol. A Collect. Rev. Nat. J. 2010, 308-319.

472. Fan, M.; Thompson, M.; Andrade, M.L.; Brolo, A.G. Silver nanoparticles on a plastic platform for localized surface plasmon resonance biosensing. Anal. Chem. 2010, 82, 6350-6352.

473. Kealley, C.S.; Arnold, M.D.; Porkovich, A.; Cortie, M.B. Sensors based on monochromatic interrogation of a localised surface plasmon resonance. Sens. Actuators B Chem. 2010, 148, 34-40.

474. Watanabe, S.; Sonobe, M.; Arai, M.; Tazume, Y.; Matsuo, T.; Nakamura, T.; Yoshida, K. Enhanced optical sensing of anions with amide-functionalized gold nanoparticles. Chem. Commun. 2002, 2866-2867. [CrossRef] 
475. Fahnestock, K.J.; Manesse, M.; McIlwee, H.A.; Schauer, C.L.; Boukherroub, R.; Szunerits, S. Selective detection of hexachromium ions by localized surface plasmon resonance measurements using gold nanoparticles/chitosan composite interfaces. Analyst 2009, 134, 881-886.

476. Kalyuzhny, G.; Vaskevich, A.; Schneeweiss, M.A.; Rubinstein, I. Transmission surface-plasmon resonance (T-SPR) measurements for monitoring adsorption on ultrathin gold island films. Chem. A Eur. J. 2002, 8, 3849-3857.

477. Tokareva, I.; Minko, S.; Fendler, J.H.; Hutter, E. Nanosensors based on responsive polymer brushes and gold nanoparticle enhanced transmission surface plasmon resonance spectroscopy. J. Am. Chem. Soc. 2004, 126, 15950-15951.

478. Englebienne, P.; Van Hoonacker, A.; Verhas, M. High-throughput screening using the surface plasmon resonance effect of colloidal gold nanoparticles. Analyst 2001, 126, 1645-1651.

479. Prabhakar, A.; Mukherji, S. A novel C-shaped, gold nanoparticle coated, embedded polymer waveguide for localized surface plasmon resonance based detection. Lab Chip 2010, 10, 3422-3425.

480. Matsui, J.; Akamatsu, K.; Hara, N.; Miyoshi, D.; Nawafune, H.; Tamaki, K.; Sugimoto, N. SPR sensor chip for detection of small molecules using molecularly imprinted polymer with embedded gold nanoparticles. Anal. Chem. 2005, 77, 4282-4285.

481. Briglin, S.M.; Gao, T.; Lewis, N.S. Detection of organic mercaptan vapors using thin films of alkylamine-passivated gold nanocrystals. Langmuir 2004, 20, 299-305.

482. Frasconi, M.; Tortolini, C.; Botre, F.; Mazzei, F. Multifunctional Au nanoparticle dendrimer-based surface plasmon resonance biosensor and its application for improved insulin detection. Anal. Chem. 2010, 82, 7335-7342.

483. Fu, E.; Ramsey, S.A.; Yager, P. Dependence of the signal amplification potential of colloidal gold nanoparticles on resonance wavelength in surface plasmon resonance-based detection. Anal. Chim. Acta 2007, 599, 118-123.

484. Hutter, E.; Pileni, M.-P. Detection of DNA hybridization by gold nanoparticle enhanced transmission surface plasmon resonance spectroscopy. J. Phys. Chem. B 2003, 107, 6497-6499.

485. Hutter, E.; Fendler, J.; Roy, D. Surface plasmon resonance studies of gold and silver nanoparticles linked to gold and silver substrates by 2-aminoethanethiol and 1, 6-hexanedithiol. J. Phys. Chem. B 2001, 105, 11159-11168.

486. Lyon, L.A.; Musick, M.D.; Natan, M.J. Colloidal Au-enhanced surface plasmon resonance immunosensing. Anal. Chem. 1998, 70, 5177-5183.

487. Pieper-Fürst, U.; Stöcklein, W.F.; Warsinke, A. Gold nanoparticle-enhanced surface plasmon resonance measurement with a highly sensitive quantification for human tissue inhibitor of metalloproteinases-2. Anal. Chim. Acta 2005, 550, 69-76.

488. Mitchell, J.S.; Lowe, T.E. Ultrasensitive detection of testosterone using conjugate linker technology in a nanoparticle-enhanced surface plasmon resonance biosensor. Biosens. Bioelectron. 2009, 24, 2177-2183.

489. Chang, Y.-F.; Chen, R.-C.; Lee, Y.-J.; Chao, S.-C.; Su, L.-C.; Li, Y.-C.; Chou, C. Localized surface plasmon coupled fluorescence fiber-optic biosensor for alpha-fetoprotein detection in human serum. Biosens. Bioelectron. 2009, 24, 1610-1614.

490. Chang, Y.-F.; Wang, S.-F.; Huang, J.C.; Su, L.-C.; Yao, L.; Li, Y.-C.; Wu, S.-C.; Chen, Y.-M.A.; Hsieh, J.-P.; Chou, C. Detection of swine-origin influenza A (H1N1) viruses using a localized surface plasmon coupled fluorescence fiber-optic biosensor. Biosens. Bioelectron. 2010, 26, 1068-1073.

491. Fang, S.; Lee, H.J.; Wark, A.W.; Corn, R.M. Attomole microarray detection of microRNAs by nanoparticle-amplified SPR imaging measurements of surface polyadenylation reactions. J. Am. Chem. Soc. 2006, 128, 14044-14046.

492. He, L.; Musick, M.D.; Nicewarner, S.R.; Salinas, F.G.; Benkovic, S.J.; Natan, M.J.; Keating, C.D. Colloidal Au-enhanced surface plasmon resonance for ultrasensitive detection of DNA hybridization. J. Am. Chem. Soc. 2000, 122, 9071-9077.

493. Yao, X.; Li, X.; Toledo, F.; Zurita-Lopez, C.; Gutova, M.; Momand, J.; Zhou, F. Subattomole oligonucleotide and p53 cDNA determinations via a high-resolution SPR combined with oligonucleotide-capped gold nanoparticle signal amplification. Anal. Biochem. 2006, 354, 220-228.

494. Bailey, R.C.; Nam, J.-M.; Mirkin, C.A.; Hupp, J.T. Real-time multicolor DNA detection with chemoresponsive diffraction gratings and nanoparticle probes. J. Am. Chem. Soc. 2003, 125, 13541-13547.

495. Du, B.A.; Li, Z.P.; Liu, C.H. One-step homogeneous detection of DNA hybridization with gold nanoparticle probes by using a linear light-scattering technique. Angew. Chem. Int. Ed. 2006, 45, 8022-8025.

496. Jans, H.; Liu, X.; Austin, L.; Maes, G.; Huo, Q. Dynamic light scattering as a powerful tool for gold nanoparticle bioconjugation and biomolecular binding studies. Anal. Chem. 2009, 81, 9425-9432.

497. Wang, X.; Xu, Y.; Xu, X.; Hu, K.; Xiang, M.; Li, L.; Liu, F.; Li, N. Direct determination of urinary lysozyme using surface plasmon resonance light-scattering of gold nanoparticles. Talanta 2010, 82, 693-697.

498. Xie, C.; Xu, F.; Huang, X.; Dong, C.; Ren, J. Single gold nanoparticles counter: An ultrasensitive detection platform for one-step homogeneous immunoassays and DNA hybridization assays. J. Am. Chem. Soc. 2009, 131, 12763-12770.

499. Wu, C.; Xiong, C.; Wang, L.; Lan, C.; Ling, L. Sensitive and selective localized surface plasmon resonance light-scattering sensor for $\mathrm{Ag}^{+}$with unmodified gold nanoparticles. Analyst 2010, 135, 2682-2687.

500. El-Sayed, I.H.; Huang, X.; El-Sayed, M.A. Surface plasmon resonance scattering and absorption of anti-EGFR antibody conjugated gold nanoparticles in cancer diagnostics: Applications in oral cancer. Nano Lett. 2005, 5, 829-834. 